\title{
Pain management for women in labour: an overview of systematic reviews (Review)
}

Jones L, Othman M, Dowswell T, Alfirevic Z, Gates S, Newburn M, Jordan S, Lavender T, Neilson $J P$

Jones L, Othman M, Dowswell T, Alfirevic Z, Gates S, Newburn M, Jordan S, Lavender T, Neilson JP.

Pain management for women in labour: an overview of systematic reviews.

Cochrane Database of Systematic Reviews 2012, Issue 3. Art. No.: CD009234.

DOI: 10.1002/14651858.CD009234.pub2.

www.cochranelibrary.com 
HEADER 1

ABSTRACT

PLAIN LANGUAGE SUMMARY

BACKGROUND

OBJECTIVES

METHODS

RESULTS

Figure 1.

Figure 2.

DISCUSSION

AUTHORS' CONCLUSIONS

ACKNOWLEDGEMENTS

REFERENCES

ADDITIONAL TABLES

APPENDICES

WHAT'S NEW

CONTRIBUTIONS OF AUTHORS

DECLARATIONS OF INTEREST

SOURCES OF SUPPORT

NOTES

INDEX TERMS

\section{TABLE OF CONTENTS}


[Overview of Reviews]

\section{Pain management for women in labour: an overview of systematic reviews}

Leanne Jones ${ }^{1}$, Mohammad Othman ${ }^{1}$, Therese Dowswell ${ }^{1}$, Zarko Alfirevic ${ }^{2}$, Simon Gates ${ }^{3}$, Mary Newburn ${ }^{4}$, Susan Jordan ${ }^{5}$, Tina Lavender6, James P Neilson ${ }^{2}$

${ }^{1}$ Cochrane Pregnancy and Childbirth Group, Department of Women's and Children's Health, The University of Liverpool, Liverpool, UK. 2Department of Women's and Children's Health, The University of Liverpool, Liverpool, UK. 3Warwick Clinical Trials Unit, Division of Health Sciences, Warwick Medical School, The University of Warwick, Coventry, UK. ${ }^{4}$ National Childbirth Trust, Acton, London, UK. ${ }^{5}$ Department of Nursing, Swansea University, Swansea, UK. ${ }^{6}$ School of Nursing, Midwifery and Social Work, The University of Manchester, Manchester, UK

Contact address: James P Neilson, Department of Women's and Children's Health, The University of Liverpool, First Floor, Liverpool Women's NHS Foundation Trust, Crown Street, Liverpool, L8 7SS, UK. jneilson@liverpool.ac.uk.

Editorial group: Cochrane Pregnancy and Childbirth Group.

Publication status and date: Edited (no change to conclusions), published in Issue 6, 2013.

Citation: Jones L, Othman M, Dowswell T, Alfirevic Z, Gates S, Newburn M, Jordan S, Lavender T, Neilson JP. Pain management for women in labour: an overview of systematic reviews. Cochrane Database of Systematic Reviews 2012, Issue 3. Art. No.: CD009234. DOI: 10.1002/14651858.CD009234.pub2.

Copyright ( 2013 The Cochrane Collaboration. Published by John Wiley \& Sons, Ltd.

\section{A B S T R A C T}

\section{Background}

The pain that women experience during labour is affected by multiple physiological and psychosocial factors and its intensity can vary greatly. Most women in labour require pain relief. Pain management strategies include non-pharmacological interventions (that aim to help women cope with pain in labour) and pharmacological interventions (that aim to relieve the pain of labour).

\section{Objectives}

To summarise the evidence from Cochrane systematic reviews on the efficacy and safety of non-pharmacological and pharmacological interventions to manage pain in labour. We considered findings from non-Cochrane systematic reviews if there was no relevant Cochrane review.

\section{Methods}

We searched the Cochrane Database of Systematic Reviews (The Cochrane Library 2011, Issue 5), The Cochrane Database of Abstracts of Reviews of Effects (The Cochrane Library 2011, Issue 2 of 4), MEDLINE (1966 to 31 May 2011) and EMBASE (1974 to 31 May 2011 ) to identify all relevant systematic reviews of randomised controlled trials of pain management in labour. Each of the contributing Cochrane reviews (six new, nine updated) followed a generic protocol with 13 common primary efficacy and safety outcomes. Each Cochrane review included comparisons with placebo, standard care or with a different intervention according to a predefined hierarchy of interventions. Two review authors extracted data and assessed methodological quality, and data were checked by a third author. This overview is a narrative summary of the results obtained from individual reviews.

\section{Main results}

We identified 15 Cochrane reviews (255 included trials) and three non-Cochrane reviews (55 included trials) for inclusion within this overview. For all interventions, with available data, results are presented as comparisons of: 1 . Intervention versus placebo or standard care; 2. Different forms of the same intervention (e.g. one opioid versus another opioid); 3. One type of intervention versus a different type of intervention (e.g. TENS versus opioid). Not all reviews included results for all comparisons. Most reviews compared the intervention with placebo or standard care, but with the exception of opioids and epidural analgesia, there were few direct comparisons between different 
forms of the same intervention, and even fewer comparisons between different interventions. Based on these three comparisons, we have categorised interventions into: " What works" ,"What may work", and "Insufficient evidence to make a judgement".

\section{WHAT WORKS}

Evidence suggests that epidural, combined spinal epidural (CSE) and inhaled analgesia effectively manage pain in labour, but may give rise to adverse effects. Epidural, and inhaled analgesia effectively relieve pain when compared with placebo or a different type of intervention (epidural versus opioids). Combined-spinal epidurals relieve pain more quickly than traditional or low dose epidurals. Women receiving inhaled analgesia were more likely to experience vomiting, nausea and dizziness.

When compared with placebo or opioids, women receiving epidural analgesia had more instrumental vaginal births and caesarean sections for fetal distress, although there was no difference in the rates of caesarean section overall. Women receiving epidural analgesia were more likely to experience hypotension, motor blockade, fever or urinary retention. Less urinary retention was observed in women receiving CSE than in women receiving traditional epidurals. More women receiving CSE than low-dose epidural experienced pruritus.

\section{WHAT MAY WORK}

There is some evidence to suggest that immersion in water, relaxation, acupuncture, massage and local anaesthetic nerve blocks or nonopioid drugs may improve management of labour pain, with few adverse effects. Evidence was mainly limited to single trials. These interventions relieved pain and improved satisfaction with pain relief (immersion, relaxation, acupuncture, local anaesthetic nerve blocks, non-opioids) and childbirth experience (immersion, relaxation, non-opioids) when compared with placebo or standard care. Relaxation was associated with fewer assisted vaginal births and acupuncture was associated with fewer assisted vaginal births and caesarean sections.

\section{INSUFFICIENT EVIDENCE}

There is insufficient evidence to make judgements on whether or not hypnosis, biofeedback, sterile water injection, aromatherapy, TENS, or parenteral opioids are more effective than placebo or other interventions for pain management in labour. In comparison with other opioids more women receiving pethidine experienced adverse effects including drowsiness and nausea.

\section{Authors' conclusions}

Most methods of non-pharmacological pain management are non-invasive and appear to be safe for mother and baby, however, their efficacy is unclear, due to limited high quality evidence. In many reviews, only one or two trials provided outcome data for analysis and the overall methodological quality of the trials was low. High quality trials are needed.

There is more evidence to support the efficacy of pharmacological methods, but these have more adverse effects. Thus, epidural analgesia provides effective pain relief but at the cost of increased instrumental vaginal birth.

It remains important to tailor methods used to each woman's wishes, needs and circumstances, such as anticipated duration of labour, the infant's condition, and any augmentation or induction of labour.

A major challenge in compiling this overview, and the individual systematic reviews on which it is based, has been the variation in use of different process and outcome measures in different trials, particularly assessment of pain and its relief, and effects on the neonate after birth. This made it difficult to pool results from otherwise similar studies, and to derive conclusions from the totality of evidence. Other important outcomes have simply not been assessed in trials; thus, despite concerns for 30 years or more about the effects of maternal opioid administration during labour on subsequent neonatal behaviour and its influence on breastfeeding, only two out of 57 trials of opioids reported breastfeeding as an outcome. We therefore strongly recommend that the outcome measures, agreed through wide consultation for this project, are used in all future trials of methods of pain management.

\section{PLAIN LANGUAGE SUMMARY}

\section{Pain management for women in labour - an overview}

Women's experience of pain during labour varies greatly. Some women feel little pain whilst others find the pain extremely distressing. A woman's position in labour, mobility, and fear and anxiety or, conversely, confidence may influence her experience of pain. Several drug and non-drug interventions are available, and in this overview we have assessed 18 systematic reviews of different interventions used to reduce pain in labour, 15 of these being Cochrane reviews.

Most of the evidence on non-drug interventions was based on just one or two studies and so the findings are not definitive. However, we found that immersion in water, relaxation, acupuncture and massage all gave pain relief and better satisfaction with pain relief. Immersion and relaxation also gave better satisfaction with childbirth. Both relaxation and acupuncture decreased the use of forceps and ventouse, with acupuncture also decreasing the number of caesarean sections. There was insufficient evidence to make a judgement on whether or not hypnosis, biofeedback, sterile water injection, aromatherapy, and TENS are effective for pain relief in labour. 
Overall, there were more studies of drug interventions. Inhaled nitrous oxide and oxygen (Entonox ${ }^{\circledR}$ ) relieved pain, but some women felt drowsy, nauseous or were sick. Non-opioid drugs (e.g. sedatives) relieved pain and some gave greater satisfaction with pain relief than placebo or no treatment, but satisfaction with pain relief was less than with opioids. Epidurals relieved pain, but increased the numbers of births needing forceps or ventouse, and the risk of low blood pressure, motor blocks (hindering leg movement), fever and urine retention. Combined spinal-epidurals gave faster pain relief but more women had itching than with epidurals alone, although urinary retention was less likely to be a problem. Local anaesthetic nerve blocks gave satisfaction but caused side effects of giddiness, sweating, tingling, and more babies had low heart rates. Parenteral opioids (injections of pethidine and related drugs) are less effective than epidural but there was insufficient evidence to make a judgement on whether or not they are more effective than other interventions for pain relief in labour.

Overall, women should feel free to choose whatever pain management they feel would help them most during labour. Women who choose non-drug pain management should feel free, if needed, to move onto a drug intervention. During pregnancy, women should be told about the benefits and potential adverse effects on themselves and their babies of the different methods of pain control. Individual studies showed considerable variation in how outcomes such as pain intensity were measured and some important outcomes were rarely or never included (for example, sense of control in labour, breastfeeding, mother and baby interaction, costs and infant outcomes). Further research is needed on the non-drug interventions for pain management in labour. 


\section{B A C K G R O U N D}

\section{History}

In 2007, $78 \%$ of the members of the Cochrane Pregnancy and Childbirth Group (PCG) consumers' group identified pain relief in labour as the topic of most importance to them. In view of the range of different interventions and the importance of the topic, the Cochrane PCG recognised that an overview of this topic was needed. In 2010 we obtained funding as part of the National Institute for Health Research (NIHR) Cochrane-NHS Engagement Scheme to produce a generic protocol for reviews examining different interventions to manage pain in labour and to produce an overview summarising the evidence from the individual reviews in a single publication. At the time of writing, 15 Cochrane reviews focus on methods to manage pain in labour. It is envisaged that the overview will provide a coherent and accessible summary of the totality of evidence about the topic, reducing or obviating the need for readers to access each individual systematic review. The generic protocol for the individual reviews has been published within The Cochrane Library.

The development of the overview protocol involved active consultation with members of the Cochrane PCG, authors of individual reviews and consumers. The methods were derived from Chapter 22 of the Cochrane Handbook of Systematic Reviews of Interventions (Higgins 2011). The list of core outcomes was developed in collaboration with members of the PCG consumers' group; 14 respondents prioritised outcomes from an extended list and added any further outcomes that were of importance to them. This revised set of outcomes was then discussed at a meeting on 4 October 2010 of stakeholders representing The Cochrane Collaboration, the Cochrane PCG, an NCT (formerly National Childbirth Trust) representative and researchers experienced in systematic reviews. A list of core outcomes was agreed. After the stakeholders' meeting, this list of core outcomes was emailed for further consideration by stakeholders and authors of individual reviews. Individual evaluations were examined and we compiled the final list of core outcomes.

\section{Description of the condition}

The pain experienced in labour is affected by the processing of multiple physiological and psychosocial factors (Lowe 2002; Simkin 2004). Perceptions of labour pain intensity vary. Very occasionally women feel no pain in labour and give birth unexpectedly (Gaskin 2003). At the other extreme labour pain has been reported to be the most severe pain that a woman experiences in her lifetime (Melzack 1984).

Pain originates from different sites during labour and birth. In the first stage of labour (defined as the period from the onset of labour to the complete dilatation of the cervix) (NLM 1991a), pain occurs during contractions, is visceral or cramp-like in nature, originates in the uterus and cervix, and is produced by distension of uterine tissues and dilation of the cervix. In the first stage, pain is transmitted via spinal nerves T10-L1. Labour pain can be referred to the abdominal wall, lumbosacral region, iliac crests, gluteal areas, and thighs. The transition phase of labour refers to the shift from the late first stage $(7 \mathrm{~cm}$ to $10 \mathrm{~cm}$ cervical dilation) to the second stage of labour (full dilation). In the second stage of labour (defined as from full cervical dilation to the delivery of the baby) (Black 2009), pain occurs from distension of the vagina, perineum, and pelvic floor. In the second stage, pain is transmitted via the pudendal nerves, entering the spinal cord via nerve roots S2-S4. Stretching of the pelvic ligaments is the hallmark of the second stage of labour. Second stage pain is characterised by a combination of visceral pain from uterine contractions and cervical stretching and somatic pain from distension of vaginal and perineal tissues. In addition, the woman experiences rectal pressure and an urge to 'push' and gives birth to her baby as the presenting part descends into the pelvic outlet.

Many factors influence the physiological and psychological processes of birth and the extent to which women experience pain, including parity and the way labour is managed. The pattern of pain, for instance, appears to be different in nulliparous as compared with multiparous women. Typically, nulliparous women experience greater sensory pain than multiparous women during early labour (before $5 \mathrm{~cm}$ dilatation) (Lowe 2002). The positions adopted by women and the extent of their mobility during labour may also significantly affect the perception of pain (Kibuka 2009; Lawrence 2009). A Cochrane systematic review (Gupta 2006) found a reduction in the reporting of severe pain during the second stage of labour for women using any upright or lateral position as compared with women lying on their back during labour. Women may also experience induced labour as being more painful than spontaneous labour (NICE 2008).

Numerous psychosocial factors also exert an influence on women's experience of labour pain. Prior experience of labour and childbirth, culture and ethnicity, educational attainment and a woman's ability to cope are often suggested as significant mediating variables on the experience of labour pain (Lowe 2002). In the last century, several philosophies of pain control evolved, using strategies to break what has been described as the fear-tensionpain cycle (Dick Read 1954; Dick Read 2004). Grantly Dick-Read, the famous advocate of 'natural childbirth', suggested that fear and anxiety can produce muscle tension, resulting in an increased perception of pain. Strategies to break the cycle of fear-tensionpain include being prepared through education and purposeful activity such as relaxation and focused breathing to relieve tension (Mozingo 1978). A wide range of 'mind-body' interventions are currently being used during pregnancy for preventing or treating women's anxiety, including autogenic training, auto-suggestion, biofeedback, hypnosis, imagery, meditation, prayer, relaxation therapy, tai chi and yoga (Marc 2011).

The physical and cultural birth environment and the degree of emotional support provided by clinical carers and the woman's birth companions also affect perceptions of pain (Foureur 2008a; Foureur 2008b). In their work with pregnant woman and expectant fathers, childbirth educators, midwives and doulas (a woman who assists women during labour and childbirth) adopt a range of different approaches to the preparation for labour and birth and ways of planning and managing labour. Leap and Anderson introduced 'the pain relief paradigm' and the 'working with pain paradigm' to theorise these different approaches (Leap 2008). The pain relief paradigm is based on a set of beliefs including the conviction that labour pain is unnecessary and barbaric in the modern world, that the benefits of analgesia outweigh the risks and women should not be made to feel guilty if they choose pain relief (Leap 2004). The working with pain paradigm is based on the view that pain is an important part of the physiology of normal labour and that, given optimal support, a woman can cope with 
levels of pain in normal labour using her own natural endorphins. Endorphins are opioids produced by the body in response to pain and other stressors. A key role for the midwife is to reduce stimulation to the woman's senses so as to facilitate endorphin release (Leap 2004).

Various multi-dimensional interventions have been shown to have an impact on the perception of pain during childbirth such as continuous support, environment and midwife-led care (Begley 2009; Hatem 2008; Hodnett 2007; Skibsted 1992). A Cochrane review of continuous support for women during childbirth found that women who had continuous intrapartum support were likely to have a slightly shorter labour, were more likely to have a spontaneous vaginal birth and less likely to have intrapartum analgesia or to report dissatisfaction with their childbirth experiences (Hodnett 2007). Another Cochrane systematic review found that women who receive midwife-led continuity of care from a small number of midwives are less likely to use pharmacological pain relief in labour, more likely to have an intervention-free labour and birth, and report an increased sense of control (Hatem 2008). Drawing together published sources of evidence, a non-Cochrane overview suggests that a trusting relationship with caregivers, continuous support, midwife-led care, preparation for labour, a home or birth centre setting and use of a birth pool are factors which make it more realistic to adopt a working with pain approach (Leap 2010).

Within the scope of the Cochrane systematic overview, we are not able to focus in detail on the many possible interactions that mediate the pain experience (spontaneous labour versus induced, primiparous versus multiparous, term versus preterm birth, continuous support versus no continuous support). Instead, we will consider these in subgroup analyses and evaluate their impact within the discussion and conclusions of the overview. The interventions that we will consider for the systematic overview have a primary focus on helping women to cope with pain in labour and in relieving pain (NICE 2007).

\section{Description of the interventions}

A wide range of pain management methods are used by women during childbirth (Caton 2002). Commonly, these include non-pharmacological interventions (hypnosis, biofeedback, intracutaneous or subcutaneous sterile water injection, immersion in water, aromatherapy, relaxation techniques (yoga, music, audio), acupuncture or acupressure, manual methods (massage, reflexology), transcutaneous electrical nerve stimulation (TENS)) and pharmacological interventions (inhaled analgesia, opioids, non-opioid drugs, local anaesthetic nerve blocks, epidural and intrathecal injections of local anaesthetics or opioids, or both). Broadly speaking, the non-pharmacological interventions primarily aim to help women cope with pain in labour, whereas the pharmacological interventions primarily aim to relieve the pain of labour (NICE 2007). However, we acknowledge that pain in labour is multifaceted and that there is obviously some overlap. Also, some interventions are taught in antenatal classes and administered prior to the onset of labour (hypnosis, biofeedback, aromatherapy, relaxation techniques (yoga, music, audio), acupuncture or acupressure, manual methods (massage, reflexology), TENS), whilst others are administered only during labour (intracutaneous or subcutaneous sterile water injection, immersion in water, inhaled analgesia, opioids, non-opioid drugs, local anaesthetic nerve blocks, epidural and intrathecal injections of local anaesthetics or opioids, or both). This issue will be explored within our discussion.

The following section outlines the range of non-pharmacological and pharmacological interventions in current use for the management of pain during childbirth.

\section{Non-pharmacological interventions}

\section{Hypnosis}

Hypnosis has been described as a state of narrow focused attention, reduced awareness of external stimuli, and an increased response to suggestions (Gamsa 2003). Suggestions are verbal or non-verbal communications that result in apparent spontaneous changes in perception, mood or behaviour. These therapeutic communications are directed to the person's subconscious and the responses are independent of any conscious effort or reasoning. Women can learn self-hypnosis which can be used in labour to reduce pain from contractions. Recent advances in neuro-imaging have led to increased understanding of the neuro-physiological changes occurring during hypnosis induced analgesia (Maquet 1999). The anterior cingulate gyrus of the limbic system has been demonstrated, by positron emission tomography, to be one of the sites in the brain affected by hypnotic modulation of pain (Faymonville 2000). The suppression of neural activity, between the sensory cortex and the amygdala-limbic system, appears to inhibit the emotional interpretation of sensations being experienced as pain.

Hypnosis for childbirth is self-hypnosis, where a practitioner teaches the mother how to induce a 'state of consciousness similar to meditation which results in failure of normally perceived experiences reaching conscious awareness' (Cyna 2004). It uses focused attention and relaxation, to develop increased receptivity to verbal and non-verbal communications which are commonly referred to as 'suggestions' (August 1961; Cyna 2004; Leap 2010; Werner 1982). These are positive statements used in order to achieve specific therapeutic goals. In labour and childbirth the goal is to alleviate or reduce fear, tension, and pain (Eng 2006; Landolt 2011) so that the physiological act of birth can progress in a way that is comfortable for the mother. There is a common misconception that when in a hypnotic state the individual loses control of her thoughts and actions, which would jeopardise their personal autonomy. Women using self-hypnosis for labour and birth are fully in control and aware of what is happening to them and those around them (August 1961).

\section{Biofeedback}

Biofeedback (or biological feedback) encompasses a therapeutic technique by which individuals receive training to improve their health and well-being through signals coming from their own bodies (including temperature, heart rate, muscular tension). The underlying principle is that changes in thoughts and emotions may result in changes in body functioning. Biofeedback aims to gain control over physiological responses with the aid of electronic instruments, under the supervision of experts. Instruments include: electromyographs measuring muscle tension; skin temperature gauges showing changes in heat emission by the skin, reflecting change in blood flow; galvanic skin response sensors, which assess the volume of sweat produced under stress by measuring skin conductivity; electroencephalographs which measure brainwave activity; electrocardiographs which monitor heart rate and rhythm 
and may be useful in detecting and relieving tachycardia (an overly rapid heartbeat) and, in turn, controlling high blood pressure. Respiration feedback devices concentrate on the rate, rhythm, and type of breathing to help lessen symptoms of asthma, anxiety, and hyperventilation, and also promote relaxation (AMA 1993; Rosenfeld 1996).

\section{Intracutaneous or subcutaneous sterile water injection}

Intracutaneous or intradermal injections of sterile water in the skin over the sacrum have been shown to relieve the pain of labour (Ader 1990; Trolle 1991; Wiruchpongsanon 2006). This technique could be of particular use to those practising in hospitals that do not have access to epidural analgesia. It could also be helpful for women who want to avoid medication during labour and birth. The technique is thought to work through the release of endogenous opioids (the endorphins and encephalins) and is based on gate control pain theories (Lytzen 1989; Trolle 1991; Wiruchpongsanon 2006).

\section{Immersion in water}

Warm water immersion during labour, including birth, used for relaxation and pain relief, has a long history in lay and clinical care (Garland 2000). It refers to the immersion in water by a pregnant woman during any stage of labour (first, second, third), and where the woman's abdomen is completely submerged. The immersion takes place in a receptacle that may be a pool, tub or bath, and which is larger than a normal domestic bath. Immersion may be for one or more stages of labour, and for any duration. The buoyancy of water enables a woman to move more easily than on land (Edlich 1987). This can facilitate the neuro-hormonal interactions of labour, alleviating pain, and potentially optimising the progress of labour (Ginesi 1998a; Ginesi 1998b). Water immersion may be associated with improved uterine perfusion, less painful contractions, and a shorter labour with fewer interventions (Aird 1997; Garland 2000; Geissbuehler 2000; Moneta 2001; Otigbah 2000; Schorn 1993). Also, shoulder-deep warm water immersion reduces blood pressure due to vasodilatation of the peripheral vessels and redistribution of blood flow. It is suggested that water immersion during labour increases maternal satisfaction and sense of control (Hall 1998; Richmond 2003). It is also suggested that the fetus benefits from a relaxed mother, as this optimises placental perfusion, and release of 'nature's opiates', the endogenous opioids (endorphins and encephalins). Accordingly, when the mother is not fearful, oxytocin release is optimised, stimulating effective contractions. In addition, the ease of mobility that water immersion offers may optimise fetal position by encouraging flexion (Ohlsson 2001).

\section{Aromatherapy}

Aromatherapy is the use of essential oils, drawing on the healing powers of plants. The mechanism of action for aromatherapy is unclear. Studies investigating psychological and physiological effects of essential oils showed no change on physiological parameters such as blood pressure or heart rate but did indicate psychological improvement in mood and anxiety (Stevensen 1995). Essential oils are thought to increase the secretion of the body's own sedative, stimulant and relaxing neurotransmitters (paracrine and endocrine). The oils may be massaged into the skin, or inhaled by using a steam infusion or burner. Aromatherapy is increasing in popularity among midwives and nurses (Allaire 2000).

\section{Relaxation techniques (yoga, music, audio)}

Relaxation techniques are mind-body interventions which are based on developing conscious awareness of muscular tension, the practice of releasing tension and maintaining relaxation often carried out in conjunction with focused breathing, meditation and visualisation. These kinds of approaches are commonly used for labour. Unanswered questions include: which approaches are most effective, the most appropriate timing for preparatory interventions during pregnancy, the extent to which practice makes a difference and which techniques women find acceptable and useful. Yoga, meditation, music and hypnosis techniques may all have a calming effect and provide a distraction from pain and tension (Vickers 1999). In future updates, this review will be split into separate reviews on yoga, music and audio.

\section{Acupuncture or acupressure}

Acupuncture involves the insertion of fine needles into different, specific parts of the body. Other acupuncture-related techniques include laser acupuncture and acupressure (applying pressure on the acupuncture point). These techniques all aim to treat illnesses and soothe pain by stimulating acupuncture points. Acupuncture points used to reduce labour pain are located on the hands, feet and ears. Several theories have been presented as to exactly how acupuncture works. One theory proposes that stimulation of touch fibres blocks pain impulses at the 'pain gates' in the spinal cord. The impulses in the pain fibres are thus less likely to reach the brain stem, thalamus and cerebral cortex (Wall 1967). Since most acupuncture points are either connected to, or located near, neural structures, this suggests that acupuncture stimulates the nervous system. Another theory suggests that acupuncture stimulates the body to release endorphins (endogenous opioids), which reduce pain (Pomeranz 1989).

\section{Massage, reflexology and other manual methods}

Manual healing methods include massage and reflexology. Massage involves manipulation of the body's soft tissues. It is commonly used to help relax tense muscles and to soothe and calm the individual. A woman who is experiencing backache during labour may find massage over the lumbosacral area soothing. Some women find light abdominal massage, known as 'effleurage', comforting. Different massage techniques may suit different women. Massage may help to relieve pain by assisting with relaxation, inhibiting sensory transmission in the pain pathways or by improving blood flow and oxygenation of tissues (Vickers 1999). Reflexologists propose that there are reflex points on the feet corresponding to organs and structures of the body and that pain may be reduced by gentle manipulation or pressing certain parts of the foot. Pressure applied to the feet has been shown to result in an anaesthetising effect on other parts of the body (Ernst 1997). In future updates, this review will be split into separate reviews on massage and reflexology.

\section{TENS}

Transcutaneous electrical nerve stimulation (TENS) uses a device which emits low voltage electrical impulses which vary in frequency and intensity. In labour, the electrodes from the TENS machine are usually attached to the lower back and women themselves control the electrical currents using a hand-held device. TENS can also be applied to acupuncture points or directly to the head by trained staff. The way that TENS acts to relieve pain is not well 
understood. The electrical pulses are thought to stimulate nerve pathways in the spinal cord which block the transmission of pain. A number of theories have been proposed. According to the gate control theory (Melzack 1965), the transmission of pain is inhibited by the stimulation of large, afferent or sensory touch nerve fibres which carry impulses towards the central nervous system. It is also suggested that painful stimuli result in release of endorphins and encephalins, which mediate the experience of pain (Lechner 1991). It is further thought that by reducing anxiety, increasing a sense of control, and by providing distraction, TENS increases a woman's sense of well-being and thereby reduces pain in labour (Brucker 1984; Findley 1999; Gentz 2001; Simkin 2004). Lastly, TENS may reduce the length of labour by suppressing the release of catecholamines, which can inhibit the contraction of the uterus and thereby, delay progress (Lowe 2002).

\section{Pharmacological interventions}

\section{Inhaled analgesia}

Inhaled analgesia during labour involves the inhalation of subanaesthetic concentrations of anaesthetic agents while the mother remains awake and her protective laryngeal reflexes remain intact. Possibilities for inhaled analgesia for pain relief in labour include isoflurane, sevoflurane, trichloroethylene in air, methoxyflurane, cyclopropane, nitrogen protoxide, nitralgin, anesoxyn and eutonal. Subanaesthetic concentrations of nitrous oxide, enflurane, isoflurane and methoxyflurane do not significantly decrease uterine contractions and are preferred for this reason. However only nitrous oxide (in 50\% oxygen) is widely used for analgesia in modern obstetric practice. This is attributed to: ease of administration, relative lack of flammability, absence of pungent odour, absence of effect on uterine contractions, lack of reports of malignant hyperthermia, minimal toxicity and minimal depression of the cardio-vascular system; a favourable partition coefficient leading to rapid onset and elimination from woman, fetus and neonate (KNOV 2009; Rosen 2002). The evidence on the use of nitrous oxide for relief of labour pain has been summarised in a systematic review (Rosen 2002). The woman can selfadminister under supervision, after initial instruction (Clyburn 1993). Inhaled analgesia is administered either intermittently, with discontinuation of use as the contraction pain eases or disappears (recommended), or continuously, by inhaling both during and between contractions. However, there is concern, centring on staff rather than patients, regarding the effect of prolonged exposure, because of reported possible associations with loss of fertility, miscarriage, preterm birth and lowered concentrations of vitamin B12 (Ahlborg 1996; Axelsson 1996; BOC 2010; Bodin 1999; Boivin 1997; Zielhuis 1999). Accordingly, nitrous oxide concentrations should be regularly measured, according to manufacturers' guidelines (BOC 2010). Other possible adverse effects are maternal drowsiness, hallucinations, vomiting, hyperventilation and tetany, and maternal or fetal hypoxia usually encountered when nitrous oxide use is excessively prolonged or extensive, especially if the rule of self-administration is violated.

The precise mechanism of action of inhaled analgesia remains uncertain, but anaesthetic actions are related to suppression of activity of the reticuloendothelial network in the brainstem. Maze and Fuginaga hypothesised that nitrous oxide induces the release of endogenous opioids in the peri-aqueductual grey area of the midbrain (Maze 2000), which could modulate pain stimuli through the descending spinal cord nerve pathways.

\section{Opioids}

Most obstetric units in developed countries offer intramuscular opioids, along with facilities for epidural analgesia. Opioids are relatively inexpensive drugs, and the use of pethidine, meptazinol or diamorphine during labour is common midwifery and obstetric practice in some countries. In other parts of the world, parenteral (intravenous or intramuscular) opioids commonly used in labour include morphine, nalbuphine, fentanyl and more recently remifentanil (Evron 2007). The extent of usage of parenteral opioids during labour worldwide is unclear. Worldwide, pethidine is the most commonly used opioid (Bricker 2002). There are concerns about maternal effects which include an impaired capacity to engage in decision making about care, sedation, hypoventilation, hypotension, prolonged labour, urine retention, nausea and/or vomiting, and the slowing of gastric emptying, which increases the risk of inhalation of gastric contents should a general anaesthetic be required in an emergency. If a woman feels drowsy or sedated, she is less likely to mobilise and adopt an upright position and, as a result, this may lengthen her labour and make it more painful (Lawrence 2009). Opioids readily cross the placenta by passive diffusion, and some are trapped by ionisation. Neonatal respiratory depression and hypothermia remain major concerns. It is estimated that it can take a newborn three to six days to eliminate pethidine, and its metabolite, norpethidine, from its system (Hogg 1977). Pethidine has been shown to significantly affect fetal heart rate variability, accelerations and decelerations, during labour (Sekhavat 2009; Solt 2002). Changes in normal fetal heart indices have consequences for the woman. She will be required to have electronic fetal heart rate monitoring if she is in hospital, and transfer to hospital if she is in the community. Results from observational studies have reported effects of opioids on the newborn that include inhibited suckling at the breast and decreased alertness, resulting in delayed effective breastfeeding (Nissen 1995; Ransjo-Arvidson 2001; Righard 1990) and earlier cessation (Rajan 1994).

\section{Non-opioid drugs}

Non-opioid medications are drugs that have principally analgesic, antipyretic, sedative and anti-inflammatory actions. They are not technically part of the analgesic family, but are nonetheless considered analgesics in practice. These include acetaminophen (paracetamol), the non-steroidal antiinflammatory drugs (NSAIDs), such as aspirin, and antispasmodic drugs such as hyoscine (Bayarski 2006; Hebbes 2000).

Acetaminophen and NSAIDs can effectively relieve mild to moderate pain, and for moderate to severe pain, they can be used in combination with other drugs to enhance pain relief.

Non-opioids affect some of the chemical changes that normally take place wherever body tissues are injured or damaged. These chemical changes at the site of the injury typically result in inflammation and increased pain sensitivity. However, there are limits to the pain afforded by non-opioids; this is referred to as a 'ceiling effect'. Once that upper limit or ceiling is reached, taking more of the non-opioid will not provide any further pain relief. Most non-opioids are quite safe when used for temporary acute pain; problems may arise when people take them over a long period of time (for chronic pain), then they could damage the lining of the gastro-intestinal tract or the kidneys, or, more rarely, other organs (Bayarski 2006; Dewhurst 2007; Hebbes 2000). 


\section{Local anaesthetic nerve blocks}

Pudendal and paracervical block (PCB) are the most commonly performed local anaesthetic nerve blocks which have been used for decades.

A pudendal block is performed by injection of local anaesthetic around the trunk of the pudendal nerve. Pudendal block is used in the second stage of labour, predominantly when instrumental delivery is performed (Pace 2004). During descent of the presenting part of the fetus in the second stage, the primary focus of pain is in the lower vagina, perineum and vulva, which are innervated from sacral nerve roots 2, 3 and 4 via the pudendal nerve. Infiltration of local anaesthetic around the trunk of the pudendal nerve at the level of ischial spines leads to analgesia of these areas. Prior to the widespread use of epidural analgesia in obstetrics, pudendal blocks were the preferred analgesic technique for delivery. Pudendal blocks are also used to supplement epidural labour analgesia, which occasionally may have some 'sacral sparing.'

A paracervical block is performed by infiltration of local anaesthetic in the cervix. It is injected into between two to six sites at a depth of $3 \mathrm{~mm}$ to $7 \mathrm{~mm}$ alongside the vaginal portion of the cervix in the vaginal fornices (Mankowski 2009). Paracervical infiltration interrupts the visceral sensory fibres of the lower uterus, cervix, and upper vagina (T10-L1) as they pass through the uterovaginal plexus (Frankenhauser's plexus) on each side of the cervix.

\section{Epidural (including combined spinal epidural)}

Epidural analgesia is a central nerve blockade technique, which involves the injection of a local anaesthetic, with or without an opioid into the lower region of the spine close to the nerves that transmit painful stimuli from the contracting uterus and birth canal. The most commonly prescribed local anaesthetic in the UK is bupivacaine; levobupivacaine, ropivacaine, and lidocaine/lignocaine are also used in epidural or intrathecal injections. Local anaesthetics inhibit nerve conduction by blocking sodium channels in nerve cell membranes, thereby preventing the propagation of nerve impulses along these fibres. Blocking impulses from the sensory nerves as they cross the epidural space results in analgesia, which should be apparent within 10 to 20 minutes of administration. The anaesthetic placed in the epidural space exerts a concentration specific effect, affecting all the modalities of sensation of the blocked nerves to varying degrees, such that administration of a lower-dose anaesthetic (e.g. $0.125 \%$ bupivacaine) partially selectively blocks painful stimuli while preserving motor function, whereas higher doses of anaesthetic cause complete sensory and motor blockade, limiting mobility in labour. The second stage of labour may be prolonged and instrumental delivery is more likely (Anim-Somuah 2005). Blocking of sympathetic nerves occurs at varying concentrations and manifests as vasodilatation and hypotension (Anim-Somuah 2005). Other reported problems include: urine retention, shivering, fever, tinnitus, tremor, respiratory and cardiovascular depression. Epidural solutions are administered either by bolus, continuous infusion or patient-controlled pump. An intermittent technique involves injections of local anaesthetic through a catheter positioned in the epidural space. Boluses of higher concentrations, as used in the earlier years, have been associated with a dense motor block resulting in reduced mobility, decreased pelvic tone and impairment of the bearing down effort in the second stage of labour (Thornton 2001). More recently, there has been a trend to use a lower concentration of local anaesthetic in combination with a variety of opiates; these combinations provide analgesic effect while allowing the woman to maintain some motor function, such as the ability to move during her labour and retain her ability to bear down (COMET 2001; Russell 2000). Combined spinalepidural (CSE) involves a single injection of local anaesthetic or opiate, or both, into the cerebral spinal fluid as well as insertion of the epidural catheter. CSE combines the advantages of spinal analgesia (faster onset of pain relief, more reliable analgesia) with the advantages of epidural analgesia such as continuing pain relief, potentially maintained throughout the entire duration of labour (Hughes 2003). However, some of the disadvantages of opioid administration remain, including itching, respiratory depression and, in observational studies, reduced breastfeeding rates (Jordan 2005; Torvaldsen 2006), but evidence is uncertain (Reynolds 2011). In addition, the rare but serious adverse effects of neuraxial administration should be considered, including introduction of infection, nerve root damage and even inadvertent intravenous injection (Jordan 2010).

\section{Why it is important to do this overview}

The totality of evidence from randomised controlled trials of interventions for pain management in labour has never been assembled before in a systematic and comprehensive way. An 'overview of reviews' will provide a clinically meaningful summary of one of the most important topics in pregnancy and childbirth. The overview provides a coherent summary of the totality of evidence without the need to access many individual systematic reviews. This may help busy clinicians, policy makers, childbirth educators and consumers.

\section{O B JECTIVES}

The objectives of this overview are to summarise the evidence from Cochrane systematic reviews regarding the effects and safety of non-pharmacological and pharmacological interventions to manage pain in labour. We also considered findings from nonCochrane systematic reviews in the absence of an available Cochrane review.

\section{METHODS}

\section{Criteria for considering reviews for inclusion}

In this overview we have included any published Cochrane systematic review of randomised controlled trials focusing on the management of pain in labour. We have only included nonCochrane systematic reviews in the absence of an available Cochrane review in an area listed below. To be considered, the non-Cochrane systematic review must have used a systematic approach, only included randomised controlled trials and have assessed the methodological quality of the included clinical trials.

The participants in reviews are women in labour. This includes women in high-risk groups, e.g. preterm labour or following induction of labour.

We have included the following non-pharmacological (hypnosis, biofeedback, intracutaneous or subcutaneous sterile water injection, immersion in water, aromatherapy, relaxation techniques (yoga, music, audio), acupuncture or acupressure, manual methods (massage, reflexology), transcutaneous electrical nerve stimulation (TENS)) and pharmacological interventions (inhaled 
analgesia, opioids, non-opioid drugs, local anaesthetic nerve blocks, epidural and intrathecal injections of local anaesthetics or opioids, or both).

We have compared interventions with placebo/no treatment or with a different intervention.

Each of the contributing Cochrane reviews followed a generic protocol (Jones 2011). To avoid duplication, each Cochrane review included comparisons only with the interventions listed above it in the following list of potential interventions. Thus, the aromatherapy review (6), from the available evidence, only included comparisons with immersion in water (5), sterile water injection (4), biofeedback (3), hypnosis (2) and placebo/no treatment (1). This strategy aimed to avoid the same comparisons being included in more than one of the original Cochrane reviews. Methods of pain management identified in the future will be added to the end of the list. The current list is as follows.

1. Placebo/no treatment.

2. Hypnosis (Madden 2012)

3. Biofeedback (Barragán 2011).

4. Intracutaneous or subcutaneous sterile water injection (Derry 2012).

5. Immersion in water (Cluett 2009).

6. Aromatherapy (Smith 2011c).

7. Relaxation techniques (yoga, music, audio) ${ }^{\star}$ (Smith 2011b).

8. Acupuncture or acupressure (Smith 2011a).

9. Massage, reflexology and other manual methods* (Smith 2012).

10.TENS (Dowswell 2009).

11.Inhaled analgesia (Klomp 2012).

12.Opioids (Ullman 2010).

13. Non-opioid drugs (Othman 2012).

14.Local anaesthetic nerve blocks (Novikova 2012).

15.Epidural (including combined spinal epidural) (Anim-Somuah 2011; Simmons 2012).

* In future updates these individual reviews will be split into separate reviews on yoga, music, audio and massage and reflexology, respectively.

\section{Outcomes}

\section{Types of outcome measure}

The following list of core outcomes was developed in collaboration with members of the Pregnancy and Childbirth Group (PCG) consumers' group - see 'History' described in Background.

\section{Primary outcomes}

\section{Effects of interventions}

Pain intensity (as defined by trialists)

Satisfaction with pain relief (as defined by trialists)

Sense of control in labour (as defined by trialists)

Satisfaction with childbirth experience (as defined by trialists)

\section{Safety of interventions}

Effect (negative) on mother/baby interaction Breastfeeding (at specified time points) Assisted vaginal birth
Caesarean section

Adverse effects (for women and infants; review specific)

Admission to special care baby unit/neonatal intensive care unit (as defined by trialists)

Apgar score less than seven at five minutes

Poor infant outcomes at long-term follow-up (as defined by trialists)

\section{Other outcomes}

Cost (as defined by trialists)

Measuring any subjective outcome is a major challenge. Pain is a highly subjective phenomenon, with a complex physiological and psychological basis (Kane 2002). It has been defined as "a complex constellation of unpleasant sensory, perceptual and emotional experiences and certain associated autonomic, psychological, emotional and behavioural responses" (Bonica 1990). Due to this complexity, there has been little research focused on developing psychometrically sound measures of pain, especially in labour and childbirth (Lowe 2002). Consequently there is considerable variation in the way that pain is measured across individual studies contained within individual reviews (Bricker 2002; Dowswell 2009). It is for these reasons that we have used the trialists' definitions of outcome measures from the individual reviews. Similarly, breastfeeding as an outcome measure is not straightforward in terms of timing of recording and reporting. The infant feeding literature offers little consistency regarding the timing of data collection. Consequently, it is difficult to compare data sets (Britton 2007). In addition, definitions of exclusive, full and partial breastfeeding will need to be considered. Where information on pain and infant feeding has been collected, we have detailed any definitions used in the results by individual review tables, in Additional tables.

\section{Search methods for identification of reviews}

We contacted the Trials Search Co-ordinator of the Cochrane Pregnancy and Childbirth Group in order to identify all relevant systematic reviews of pain management in labour. In the absence of an available Cochrane systematic review in one of the following areas (hypnosis, biofeedback, intracutaneous or subcutaneous sterile water injection, immersion in water, aromatherapy, relaxation techniques (yoga, music, audio), acupuncture or acupressure, manual methods (massage, reflexology), TENS, inhaled analgesia, opioids, non-opioids, local anaesthetic nerve blocks, epidural), we searched the Database of Abstracts of Reviews of Effects (The Cochrane Library 2011, Issue 2 of 4), MEDLINE (1966 to 31 May 2011) and EMBASE (1980 to 31 May 2011) using the search strategies detailed in Appendix 1.

\section{Data collection and analysis}

The methodology for data collection and analysis is based on Chapter 22 of the Cochrane Handbook of Systematic Reviews of Interventions (Higgins 2011).

\section{Selection of reviews}

Two review authors independently assessed for inclusion all the potential systematic reviews we identified as a result of the search strategy. We resolved any disagreement through discussion or, if required, we consulted a third person. 


\section{Data extraction and management}

Two review authors independently extracted data from the reviews using a predefined data extraction form. We resolved discrepancies through discussion or, if required, we consulted a third person. We entered data into Review Manager software (RevMan 2011) and checked for accuracy. If any information from the reviews was unclear or missing, we accessed the published reports of the individual trials. If the information could not be obtained from the published reports, then we contacted the review authors or authors of the original reports to provide clarification and further details.

\section{Assessment of methodological quality of included reviews}

We have addressed two different quality assessments in this overview: the quality of evidence in the included reviews and the methodological quality of the systematic reviews. Two review authors assessed methodological quality independently. We resolved discrepancies through discussion or, if required, we consulted a third person.

\section{Quality of evidence in included reviews}

Two review authors independently assessed the overall quality of the evidence presented in the included reviews by examining the methods used for assessing risk of bias of the individual included studies. We assessed whether the Cochrane reviews used the domain-based evaluation for assessment of risk of bias as outlined in Chapter 8 of the Cochrane Handbook for Systematic Reviews of Interventions (Higgins 2011). For non-Cochrane systematic reviews, we have summarised the methods used to assess methodological quality, including details regarding the tools used and the dimensions assessed e.g. sequence generation; allocation sequence concealment; blinding; incomplete outcome data.

\section{Quality of included reviews}

Two review authors independently assessed the methodological quality of the included reviews using the 'assessment of multiple systematic reviews' (AMSTAR) measurement tool (Shea 2007). The AMSTAR tool assesses the following criteria.

1. Was an 'a priori' design provided?

2. Was there duplicate study selection and data extraction?

3. Was a comprehensive literature search performed?

4. Was the status of publication (i.e. grey literature) used as an inclusion criterion?

5. Was a list of studies (included and excluded) provided?

6. Were the characteristics of the included studies provided?

7. Was the scientific quality of the included studies assessed and documented?

8. Was the scientific quality of the included studies used appropriately in formulating conclusions?

9. Were the methods used to combine the findings of studies appropriate?

10.Was the likelihood of publication bias assessed?

\section{Was the conflict of interest stated?}

\section{Data synthesis}

We have provided a narrative summary of the results for the individual reviews for each of the primary outcomes and present these using tables and figures (e.g. characteristics of included reviews, summary of quality of evidence within individual systematic reviews, AMSTAR ratings for each systematic review, results by individual review tables). It was not anticipated that we would be able to perform any quantitative data analyses. However, for future updates of this overview, if the data allow, we may perform some indirect comparisons of interventions across reviews for the primary outcomes. We had planned, if possible, to present data from the following subgroups (if these data were available within the included systematic reviews).

1. Spontaneous labour versus induced labour.

2. Primiparous versus multiparous.

3. Term versus preterm birth.

4. Continuous support in labour versus no continuous support.

\section{RESULTS}

\section{Cochrane systematic reviews}

A total of 15 Cochrane systematic reviews were identified by the Trials Search Co-ordinator of the Cochrane Pregnancy and Childbirth Group, all of which met the inclusion criteria for this overview. A priori, the research question and inclusion criteria were provided in a published generic protocol (Jones 2011). All but one of the 15 Cochrane systematic reviews (protocols and updates of reviews), followed this generic protocol. The Cochrane review on combined spinal-epidural versus epidural analgesia in labour (Simmons 2012) did not adhere to the generic protocol because it did not fit in with the hierarchy of interventions.

\section{Non-Cochrane systematic reviews}

In order to identify any gaps not already covered by the Cochrane systematic reviews, we searched The Database of Abstracts of Reviews of Effects (The Cochrane Library 2011, Issue 2 of 4), MEDLINE (1966 to 31 May 2011) and EMBASE (1980 to 31 May 2011) using the search strategies detailed in Appendix 1. A total of 65 potentially eligible reviews were identified from this search. Three of these filled a gap not already covered by the Cochrane reviews (Halpern 2003a; Hutton 2009; Mardirosoff 2002). The remaining 62 were excluded because they were not a systematic review $(\mathrm{N}=26)$, they were out of date and focused on an area already covered by one of the included Cochrane or non-Cochrane systematic reviews $(\mathrm{N}=32)$, or they did not fit the inclusion criteria for this overview ( $\mathrm{N}$ $=4)$. A description of the characteristics of these excluded reviews, and the reasons for exclusion are set out in an additional table (Table 1).

Figure 1 gives a flow diagram outlining the selection process and review numbers at each stage. 
Figure 1. Study flow diagram.

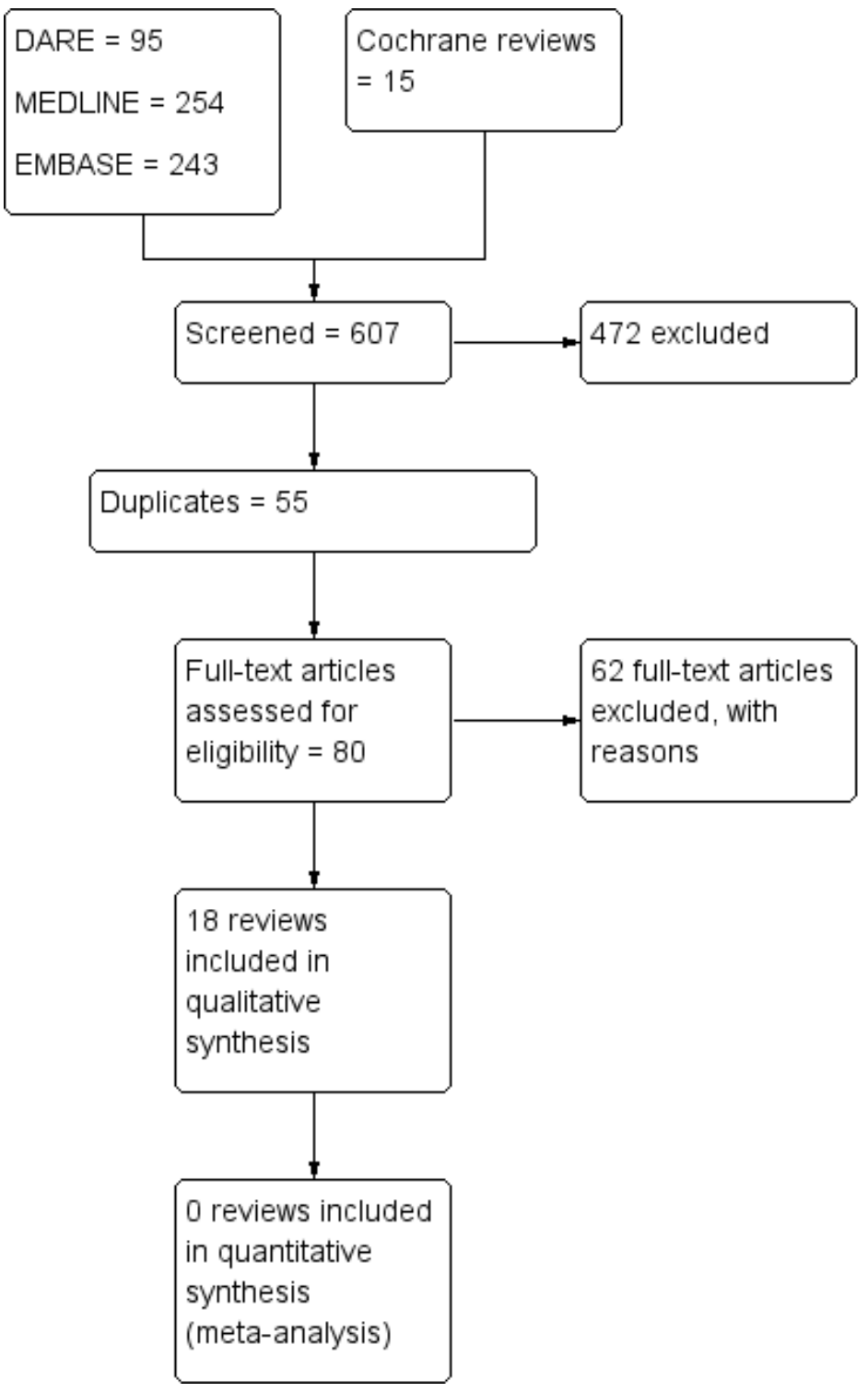

\section{Description of included reviews}

We have included 15 Cochrane reviews: nine non-pharmacological reviews (hypnosis (Madden 2012), biofeedback (Barragán 2011), intracutaneous or subcutaneous sterile water injection (Derry 2012), immersion in water (Cluett 2009), aromatherapy (Smith 2011c), relaxation techniques [yoga, music, audio] (Smith 2011b), acupuncture or acupressure (Smith 2011a), manual methods (massage, reflexology) (Smith 2012), transcutaneous electrical nerve stimulation (TENS) (Dowswell 2009)) and six examining pharmacological interventions (inhaled analgesia (Klomp 2012), opioids (Ullman 2010), non-opioid drugs (Othman 2012), local anaesthetic nerve blocks (Novikova 2012), epidural and intrathecal injections of local anaesthetics or opioids, or both (Anim-Somuah 2011; Simmons 2012)). All of these reviews have recently been updated and the search dates are in 2011. Eleven of the reviews had already been published or were in-press at the time this overview was prepared. However, while four of the remaining reviews had been submitted for publication at this time, they had not been revised after peer review, or approved for publication. Therefore, the findings we have reported in this overview for these reviews (examining hypnosis (Madden 2012), inhaled analgesia (Klomp 2012), non-opioid drugs (Othman 2012) and combined spinal epidural (Simmons 2012)) are based on the draft reviews submitted by the authors for editorial consideration. We are aware that including data from these non-completed reviews potentially introduces bias into the overview process. If there are changes in results in of any of these reviews, new findings will be incorporated into the first update of this overview.

The titles of the 15 Cochrane reviews are listed below:

1. Hypnosis for pain management during labour and childbirth (Madden 2012).

2. Biofeedback for pain management during labour (Barragán 2011). 
3. Intracutaneous or subcutaneous sterile water injection for pain management in labour (Derry 2012).

4. Immersion in water in labour and birth Cluett 2009).

5, Aromatherapy for pain management in labour (Smith 2011c).

6. Relaxation techniques for pain management in labour (Smith 2011b).

7. Acupuncture or acupressure for pain management in labour (Smith 2011a).

8. Massage, reflexology and other manual methods for pain management in labour (Smith 2012).

9. Transcutaneous electrical nerve stimulation (TENS) for pain management in labour (Dowswell 2009).

10. Inhaled analgesia for pain management in labour (Klomp 2012).

11. Parenteral opioids for maternal pain management in labour (Ullman 2010).

12. Non-opioid drugs for pain management in labour (Othman 2012).

13. Local anaesthetic nerve block for pain management in labour (Novikova 2012).

14. Epidural versus non-epidural or no analgesia in labour (AnimSomuah 2011).

15. Combined spinal-epidural versus epidural analgesia in labour (Simmons 2012).

In addition we have included three non-Cochrane reviews that focused on methods of pain relief or comparisons not covered by the Cochrane reviews (Halpern 2003a; Hutton 2009; Mardirosoff 2002) and the dates of publication indicate that these reviews are less up to date. These reviews examined sterile water injections (Hutton 2009); intrathecal opioids (Mardirosoff 2002) and epidural ropivacaine versus bupivacaine (Halpern 2003a), as listed:

1. Sterile water injection for labour pain: a systematic review and meta-analysis of randomised controlled trials (Hutton 2009).

2. Fetal bradycardia due to intrathecal opioids for labour analgesia: a systematic review (Mardirosoff 2002).

3. Epidural ropivacaine versus bupivacaine for labor: a metaanalysis (Halpern 2003a).

The specific inclusion and exclusion criteria in the contributing reviews varied: all focused on pain in labour and all included randomised trials; however, authors of each review had particular criteria for participants and interventions. For example, in the hypnosis review women may have been recruited and the intervention may have occurred during pregnancy (for example in antenatal classes), whereas, for pharmacological interventions women were predominantly recruited when they were admitted to hospital during labour. While all but one (combined spinal epidural) of the Cochrane reviews were based on a generic protocol and collected outcome data on a prespecified list of primary and secondary outcomes, individual review authors may also have collected data on other outcomes which we have not reported in this overview. Outcomes relating to adverse effects are mainly review (i.e. intervention) specific. For non-Cochrane reviews outcomes were determined by the individual review authors and may not have included most of our prespecified outcomes; for this reason we have reported only limited data from these nonCochrane reviews.

The number of trials and participants included in the various contributing reviews varied considerably from two studies and 535 women in the aromatherapy review, and four studies and 201 women in the biofeedback review, through to 57 included studies and more than 7000 participants in the parenteral opioids review. The trials in each of the contributing reviews were carried out in a variety of settings and over varying periods of time; for example, epidural has been used widely since the 1980 s whereas studies included in the parenteral opioids review date back as far as the 1930s.

We have set out the characteristics of the contributing reviews in four additional tables and it is important that the findings of the overview are interpreted in the light of information in the tables.

Characteristics of included Cochrane systematic reviews - nonpharmacological interventions - see Table 2

Characteristics of included Cochrane systematic reviews pharmacological interventions - see Table 3

Characteristics of included Non-Cochrane systematic reviews non-pharmacological interventions - see Table 4

Characteristics of included Non-Cochrane systematic reviews pharmacological interventions - see Table 5

\section{Quality of evidence in included reviews}

The quality of the evidence (i.e. the methodological quality of individual trials) in each of the contributing reviews was assessed using the Cochrane risk of bias tool set out in the Handbook (Higgins 2011). Just as the number of trials contributing data to each review varied, so too did the overall methodological quality of those trials. Again, the evidence in each section of the results should be interpreted in the light of the strengths and weaknesses of individual trials. In the Cochrane reviews focusing on non-pharmacological interventions, for example, blinding staff and participants in trials was either not attempted, or it was not clear that alternatives (such as sham TENS devices) achieved successful blinding. In the Cochrane review focusing on sterile water injection trials were only included if they were double-blind, but this criterion was not used in all reviews. Many trials included in reviews used methods to generate the randomisation sequence, and to allocate participants to groups, with high risk of bias. For example, in the TENS review, most of the included studies used methods of allocating participants that did not conceal allocations and therefore were at high risk of bias. Many trials involving pharmacological methods could achieve blinding through use of placebo, but these trials often encountered other methodological problems (for example high levels of sample attrition or protocol deviations) leaving results at high risk of bias. The quality of the evidence from Cochrane reviews has been summarised in two additional tables:

Quality of evidence in included Cochrane systematic reviews - nonpharmacological interventions - see Table 6 
Quality of evidence in included Cochrane systematic reviews pharmacological interventions - see Table 7

The same issues regarding the strengths and weaknesses of the evidence in non-Cochrane reviews apply. However, as these reviews did not use the Cochrane domain based risk of bias tool we were unable to assess the overall quality of contributing studies. The quality of evidence in the three non-Cochrane review are set out in additional tables:

Quality of evidence in included Non-Cochrane systematic reviews non-pharmacological interventions - see Table 8.

Quality of evidence in included Non-Cochrane systematic reviews - pharmacological interventions - see Table 9

\section{Methodological quality of included reviews}

The methods used in Cochrane reviews were assessed using the AMSTAR rating scale described above. As all Cochrane reviews followed a generic protocol specifying methods, scores were high for all reviews. The Cochrane Handbook for Systematic Reviews of Interventions specifies that the search should be comprehensive; data extraction should be carried out independently by two people; methods for data synthesis should be specified; reasons for excluding studies and characteristics of those included should be described; the quality of included studies should be assessed; and data should be analysed and findings reported appropriately. These are all factors which were adhered to in all contributing
Cochrane reviews and which contribute to achieving positive AMSTAR ratings. In addition, all review protocols go through a peer review process before publication when methodological quality is assessed. So, it was not surprising that all these reviews received high-quality ratings. For the non-Cochrane reviews scores were less high; this was generally because some aspects of the review process may not have been explicit in the published reviews. Findings regarding the quality of contributing reviews are set out in three additional tables.

AMSTAR ratings for each Cochrane systematic review - nonpharmacological interventions - see Table 10 AMSTAR ratings for each Cochrane systematic review pharmacological interventions - see Table 11

AMSTAR ratings for each Non-Cochrane systematic review - see Table 12

\section{Effect of interventions}

For all interventions, where separate data were available, we have set out the results under three major comparison groups:

1. Intervention versus placebo or standard care;

2. different forms of the same intervention (e.g. one opioid versus another opioid); and,

3. one type of intervention versus a different type of intervention (e.g. TENS versus opioid), see Figure 2.

\section{Figure 2. Summary of comparisons within included reviews}

\begin{tabular}{|c|c|c|c|c|c|c|c|c|c|c|c|c|c|c|c|}
\hline METHODS & & & & & & JMBEF & OF TRI & ALS (NL & MBER & OF WO & (EN) & & & & \\
\hline Placebo/no treatment & & $\begin{array}{c}7 \\
\text { (1213) }\end{array}$ & $\begin{array}{c}4 \\
\text { (201) }\end{array}$ & $\begin{array}{c}6 \\
(667)\end{array}$ & $\begin{array}{c}11 \\
(3052)\end{array}$ & $\begin{array}{c}1 \\
(513)\end{array}$ & $\begin{array}{c}11 \\
(1824)\end{array}$ & $\begin{array}{c}12 \\
(1858)\end{array}$ & $\begin{array}{c}4 \\
(225)\end{array}$ & $\begin{array}{c}17 \\
(1455)\end{array}$ & $\begin{array}{c}9 \\
(1495)\end{array}$ & $\begin{array}{c}3 \\
(226)\end{array}$ & $\begin{array}{c}14 \\
(2003)\end{array}$ & $\begin{array}{c}1 \\
(200)\end{array}$ & $\begin{array}{c}5 \\
(790)\end{array}$ \\
\hline Hypnosis & $\begin{array}{c}7 \\
(1213)\end{array}$ & $\begin{array}{l}0 \\
(0)\end{array}$ & & & & & & & & & & & & & \\
\hline Biofeedback & $\begin{array}{c}4 \\
(201)\end{array}$ & & $\begin{array}{c}0 \\
(0)\end{array}$ & & & & & & & & & & & & \\
\hline Sterile water injection & $\begin{array}{c}6 \\
(667)\end{array}$ & & & $\begin{array}{c}1 \\
(99)\end{array}$ & & & & $\begin{array}{c}1 \\
(128)\end{array}$ & & $\begin{array}{c}1 \\
(22)\end{array}$ & & & & & \\
\hline Immersion in water & $\begin{array}{c}11 \\
\text { (3052) }\end{array}$ & & & & $\begin{array}{c}1 \\
200\end{array}$ & & & & & & & & & & \\
\hline Aromatherapy & $\begin{array}{c}1 \\
(513)\end{array}$ & & & & & $\begin{array}{c}1 \\
(22)\end{array}$ & & & & & & & & & \\
\hline Relaxation Techniques & $\begin{array}{c}11 \\
(1824)\end{array}$ & & & & & & $\begin{array}{c}0 \\
(0)\end{array}$ & & $\begin{array}{c}2 \\
(129)\end{array}$ & & & & & & \\
\hline Acupuncture/ acupressure & $\begin{array}{c}12 \\
(1858)\end{array}$ & & & $\begin{array}{c}1 \\
(128)\end{array}$ & & & & $\begin{array}{c}0 \\
(0)\end{array}$ & & & & & & & \\
\hline Manual methods & $\begin{array}{c}4 \\
(225)\end{array}$ & & & & & & $\begin{array}{c}2 \\
(129)\end{array}$ & & $\begin{array}{c}0 \\
(0)\end{array}$ & & & & & & \\
\hline TENS & $\begin{array}{c}17 \\
(1455)\end{array}$ & & & $\begin{array}{c}1 \\
(22)\end{array}$ & & & & & & $\begin{array}{c}0 \\
(0)\end{array}$ & $\begin{array}{c}1 \\
(20)\end{array}$ & $\begin{array}{c}3 \\
(305)\end{array}$ & & & \\
\hline Inhaled analgesia & $\begin{array}{c}9 \\
(1495)\end{array}$ & & & & & & & & & $\begin{array}{c}1 \\
(20)\end{array}$ & $\begin{array}{c}14 \\
(752)\end{array}$ & & & & \\
\hline Parenteral opioids & $\begin{array}{c}3 \\
(226)\end{array}$ & & & & & & & & & $\begin{array}{c}3 \\
(305)\end{array}$ & & $\begin{array}{c}53 \\
(7534)\end{array}$ & $\begin{array}{c}3 \\
(563)\end{array}$ & $\begin{array}{c}2 \\
(229)\end{array}$ & $\begin{array}{c}33 \\
(8868)\end{array}$ \\
\hline Non-opioid drugs & $\begin{array}{c}14 \\
(2003)\end{array}$ & & & & & & & & & & & $\begin{array}{c}3 \\
(563)\end{array}$ & $\begin{array}{c}3 \\
(590)\end{array}$ & $\begin{array}{c}1 \\
(100)\end{array}$ & \\
\hline Local anaesthetic & $\begin{array}{c}1 \\
(200)\end{array}$ & & & & & & & & & & & $\begin{array}{c}2 \\
(229)\end{array}$ & $1(100)$ & $\begin{array}{c}8 \\
(1120)\end{array}$ & \\
\hline Epidural and CSE & $\begin{array}{c}5 \\
(790) \\
\end{array}$ & & & & & & & & & & & $\begin{array}{c}33 \\
(8868) \\
\end{array}$ & & & $\begin{array}{c}27 \\
(3303) \\
\end{array}$ \\
\hline COMPARISONS & 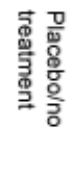 & $\begin{array}{l}\frac{T}{2} \\
\frac{\partial}{\partial} \\
\frac{0}{\omega} \\
\frac{n}{\omega}\end{array}$ & 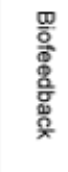 & 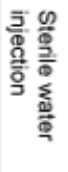 & 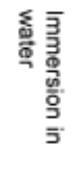 & 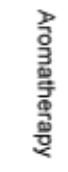 & 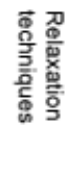 & 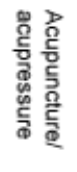 & 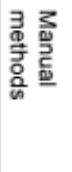 & $\underset{\text { m }}{\text { m }}$ & 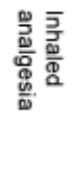 & 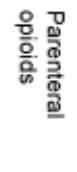 & 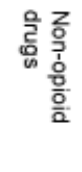 & 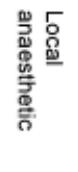 & 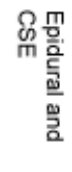 \\
\hline
\end{tabular}

KEY

$=$ Intervention versus placebo or standard care

= Different forms of the same intervention (e.g. one opioid versus another opioid)

= One type of intervention versus a different type of intervention (e.g. TENS versus sterile water) 
Not all contributing reviews included results for all three types of comparisons. Most reviews compared the intervention with placebo or no treatment. However, with the exceptions of parenteral opioids and different types of epidural, there were few direct comparisons between different forms of the same intervention, and even fewer instances of different interventions being compared with each other, see Figure 2.

For each review, we have set out the overall number of studies and total number of women randomised. We have also provided the number of studies and numbers of women randomised to each different comparison. These two sets of numbers may differ, as some trials included more than two arms and may be included in more than one comparison. We have also specified the number of studies and women at the outcome level: for many outcomes only a small number of studies contributed data.

\section{Cochrane Systematic reviews}

\section{Non-pharmacological interventions (nine reviews)}

\section{Hypnosis for pain management during labour and childbirth (seven studies)}

Seven studies randomising 1213 women were included in this review (Madden 2012): one of the included studies included three arms (hypnosis versus another type of hypnosis versus standard care, 448 women) and data from the hypnosis versus standard care arms are included in comparison 1.1. and data from the hypnosis versus different hypnosis arm has been included in comparison 1.2.

\subsection{Hypnosis versus no hypnosis/ standard care (seven studies, 1070 women)}

For this comparison control group interventions varied, and it was not always clear whether the experimental intervention was offered as an addition, or as an alternative to routine childbirth preparation. There were some studies that did not contribute data to any outcomes.

There was no evidence of a significant difference in satisfaction with pain relief (risk ratio (RR) 1.06, 95\% confidence interval $(\mathrm{Cl}) 0.94$ to 1.20, one trial, 264 women); satisfaction with childbirth experience (RR $1.36,95 \% \mathrm{Cl} 0.52$ to 3.59 , two trials, 370 women); assisted vaginal birth ( $\mathrm{RR} 0.73,95 \% \mathrm{Cl} 0.36$ to 1.50 , three trials, 414 women); caesarean section (RR $0.54,95 \% \mathrm{Cl} 0.18$ to 1.62 , three trials, 867 women) or admission to neonatal intensive care unit (RR 0.58, 95\% $\mathrm{Cl} 0.12$ to 2.83 , two trials, 345 women). There was no evidence of a significant difference for any of the other outcomes analysed (breastfeeding; adverse effects for women or infants; Apgar score less than seven at five minutes).

None of the following outcomes were analysed within the review: sense of control in labour; effect on mother/baby interaction; poor infant outcomes at long-term follow-up; or cost.

\subsection{One type of hypnosis versus another type of hypnosis (one study, 297 women)}

When hypnosis administered by a hypnotherapist was compared with hypnosis on an audio CD administered by a nurse without training in hypnotherapy, there were no significant differences for any of the outcomes analysed (satisfaction with pain relief; satisfaction with childbirth experience; breastfeeding; assisted vaginal birth; caesarean section; adverse effects for women and infants; admission to neonatal intensive care unit; Apgar score less than seven at five minutes).

None of the following outcomes were analysed within the review: sense of control in labour; effect on mother/baby interaction; poor infant outcomes at long-term follow-up; or cost.

\subsection{Hypnosis versus a different intervention}

No studies are included in this comparison.

See Table 13 for all results relating to hypnosis.

\section{Biofeedback for pain management during labour (four studies)}

\subsection{Biofeedback versus no biofeedback/ standard care (four studies,} 201 women)

Four studies randomising 201 women were included in this review (Barragán 2011): the type of biofeedback and the childbirth preparation received by women in control arms varied in these studies. The data relating to pain intensity, satisfaction with pain relief, sense of control in labour or Apgar score were not reported in a format that could be included in any analysis.

There was no significant difference between groups comparing biofeedback with control for assisted vaginal birth (average RR $0.75,95 \% \mathrm{Cl} 0.18$ to 3.10 , two trials, 103 women (random effects; heterogeneity: $\mathrm{I}^{2}=80 \%, \mathrm{Tau}^{2}=0.86, \mathrm{Chi}^{2}$ test for heterogeneity $\mathrm{P}=$ 0.02 ) or caesarean section (RR $0.41,95 \% \mathrm{Cl} 0.14$ to 1.15 , two trials, 103 women).

None of the following outcomes were analysed within the review: satisfaction with childbirth experience; effect on mother/baby interaction; breastfeeding; adverse effects for women and infants; admission to special care baby unit/neonatal intensive care unit; poor infant outcomes at long-term follow-up; or cost.

\subsection{One type of biofeedback versus a different type of biofeedback}

No studies examined this comparison.

\subsection{Biofeedback versus a different intervention}

No studies examined this comparison.

See Table 14 for all results on biofeedback.

\section{Intracutaneous or subcutaneous sterile water injection for pain management in labour (seven studies)}

Seven studies randomising 766 women were included in this review (Derry 2012): four studies compared intracutaneous sterile water injections versus placebo (saline injections) (467 women); two studies compared subcutaneous sterile water injection versus placebo (saline injection) (200 women); and one study compared intracutaneous or subcutaneous sterile water injection versus placebo (saline injection) (99 women).

\subsection{Intracutaneous or subcutaneous sterile water injection versus placebo (blinded controls) for pain management in labour (six studies, 667 women)}

Pain intensity: Although pain intensity was reported in several of the studies contributing data to this review, the review authors did not consider that data were presented in a way that was 
suitable for inclusion in meta-analysis, or that was clinically meaningful. Therefore, in this overview we have not set out results for this outcome. Pain intensity data were, however, reported in an included non-Cochrane review focusing on sterile water injection described below.

There was no evidence of a significant difference in assisted vaginal births (RR $1.31,95 \% \mathrm{Cl} 0.79$ to 2.18 , six trials, 666 women) or caesarean section (RR $0.58,95 \% \mathrm{Cl} 0.33$ to 1.02 , seven trials, 766 women) between the sterile water and placebo groups.

None of the following outcomes were analysed within the review: satisfaction with pain relief; sense of control in labour; satisfaction with childbirth experience; effect on mother/baby interaction; breastfeeding; adverse effects for women or infants; admission to neonatal intensive care unit; Apgar score less than seven at five minutes; poor infant outcomes at long-term follow-up; or cost.

\subsection{Intracutaneous versus subcutaneous sterile water injection (one study, 99 women)}

Pain intensity: Although pain intensity was reported in the single study examining this comparison, review authors considered that data were either unsuitable for inclusion in meta-analysis, or were not clinically meaningful. Therefore, in this overview we have not set out results for this outcome.

\subsection{Intracutaneous water versus a different intervention}

One study compared TENS with sterile water injection and one examined sterile water versus acupuncture (Dowswell 2009). These studies have been discussed in the relevant sections below.

See Table 15 for all results relating to sterile water injections.

\section{Immersion in water in labour and birth (12 studies)}

Twelve studies randomising 3252 women were included in this review (Cluett 2009). Altogether 11 studies compared immersion versus no immersion (3052 women); eight compared immersion versus no immersion in the first stage of labour ( 2766 women); one study compared immersion versus no immersion in the second stage of labour ( 120 women) and two studies compared immersion versus no immersion in both the first and second stages of labour (166 women). One study compared early $(<5 \mathrm{~cm}$ dilation) versus late ( $>=5 \mathrm{~cm}$ dilation) immersion during the first stage of labour (200 women). We have presented results separately for those studies comparing immersion (early or late) versus no immersion as opposed to the single study comparing immersion at different stages during labour.

\subsection{Immersion in water during the first or second stage of labour} versus no immersion in water/ standard care (11 studies, 3052 women)

Overall, there was little difference between groups for most of the comparisons including: breastfeeding, assisted vaginal birth, caesarean section, adverse effects for infants, admission to special care baby unit and Apgar score less than seven at five minutes. The only differences observed between groups were for pain intensity, adverse effects for mothers, and satisfaction with childbirth experience. For all of these outcomes evidence was derived from single studies.

Pain intensity: In a single trial ( 120 women) comparing immersion versus no immersion in the first stage of labour significantly fewer women in the immersion group reported their pain intensity as being moderate to severe at 30 minutes after randomisation on three different instruments measuring pain intensity (RR 0.75, 95\% $\mathrm{Cl} 0.62$ to 0.91 ; RR $0.72,95 \% \mathrm{Cl} 0.58$ to 0.90 ; RR $0.67,95 \% \mathrm{Cl} 0.51$ to $0.90)$. At one hour and two hours after randomisation fewer women in the immersion group reported moderate or severe pain for two out of the three ordinal scales (one hour - RR $0.76,95 \% \mathrm{Cl} 0.63$ to 0.91 ; RR $0.68,95 \% \mathrm{Cl} 0.53$ to 0.86 ) (two hours - RR $0.76,95 \% \mathrm{Cl}$ 0.59 to 0.98 ; $\mathrm{RR} 0.72,95 \% \mathrm{Cl} 0.52$ to 0.98 ). There were no significant differences between groups at three hours after randomisation on any of the three ordinal scales.

Adverse effects for women: In one trial that compared immersion versus no immersion in the first stage of labour mean systolic, diastolic and arterial blood pressures were significantly lower in women in the immersion group, (mean difference (MD) $-7.20,95 \%$ $\mathrm{Cl}-13.12$ to -1.28 ; MD $-10.20,95 \% \mathrm{Cl}-13.70$ to -6.70 ; MD -10.50, 95\% $\mathrm{Cl}-14.68$ to $-6.32,120$ women).

Satisfaction with childbirth experience: Of the three trials that compared water immersion during the second stage with no immersion, one trial (117) showed that significantly fewer women in the immersion group reported low satisfaction (reported as did not cope with pushing efforts) with the childbirth experience (RR $0.24,95 \% \mathrm{Cl} 0.07$ to 0.80 ).

None of the following outcomes were analysed in the review: satisfaction with pain relief; sense of control in labour; effect on mother/baby interaction; poor infant outcomes at long-term follow-up; or cost.

\subsection{Immersion in water during early compared with later in labour (one study 200 women)}

Results were reported for two outcomes: adverse effects in infants and Apgar scores at one minute; there was no significant difference between groups for either of these outcomes. Results for other outcomes were not reported.

\subsection{Immersion in water versus a different intervention}

No studies examined this comparison.

See Table 16 for all results relating to immersion in water.

\section{Aromatherapy for pain management in labour (two studies)}

Two studies randomising 535 women were included in this review (Smith 2011c): one study compared aromatherapy versus standard care (513 women); and one study compared aromatherapy using ginger versus aromatherapy using lemon grass (22 women). None of the data relating to pain assessment were reported in a way that allowed the review authors to include them in the analysis. In one study, data were reported for only one arm of the trial and in the other median values were presented.

\subsection{Aromatherapy versus standard care (one study, 513 women)}

There was no evidence of a significant difference between groups comparing aromatherapy with standard care for assisted vaginal birth or caesarean section (RR $1.04,95 \% \mathrm{Cl} 0.48$ to 2.28 , and RR $0.98,95 \% \mathrm{Cl} 0.49$ to 1.94 respectively; one trial, 513 women). There was also no evidence of a significant difference between groups for admission to neonatal intensive care unit (NICU) (RR 0.08, 95\% Cl 0.00 to 1.42 , one trial, 513 women). 
5.2. Aromatherapy (ginger) versus aromatherapy (lemon grass) (one study, 22 women)

There was no evidence of a significant difference between groups comparing different types of aromatherapy for assisted vaginal birth or caesarean section (RR $0.83,95 \% \mathrm{Cl} 0.06$ to 11.70 , and RR $2.54,95 \% \mathrm{Cl} 0.11$ to 56.25 respectively, one trial, 22 women). No women in either group had a postpartum haemorrhage and no babies were admitted to NICU (one trial, 22 women).

None of the following outcomes were analysed within the review: satisfaction with pain relief; sense of control in labour; satisfaction with childbirth experience; effect on mother/baby interaction; breastfeeding; adverse effects for infants; Apgar score less than seven at five minutes; poor infant outcomes at long-term follow-up; or cost.

\subsection{Aromatherapy versus a different intervention}

No studies examined this comparison

See Table 17 for all results on aromatherapy.

\section{Relaxation techniques for pain management in labour (11 studies)}

\subsection{Relaxation techniques versus standard care (11 studies)}

Eleven studies involving 1574 women were included in this review (Smith 2011b): six studies compared relaxation in the form of progressive muscle relaxation, breathing or psycho-prophylaxis versus standard care (1147 women); two studies compared yoga versus standard care (270 women); two studies compared music versus standard care (133 women); and one study compared audio-analgesia versus standard care (24 women). There was considerable variation in these studies in the way pain, pain relief and satisfaction with the childbirth experience were measured.

Pain intensity: Five of the 11 included trials reported data on pain intensity as continuous data. A significant difference was observed between groups in studies comparing relaxation with standard care in both the latent and active phases of labour (MD $-1.25,95 \% \mathrm{Cl}$ -1.97 to -0.53 , one trial, 40 women; MD $-2.48,95 \% \mathrm{Cl}-3.13$ to -1.83 , two trials, 74 women) and in one study comparing yoga versus standard care in the latent phase of labour (MD -6.12, 95\% Cl-11.77 to -0.47 , one trial, 66 women), with a reduction in pain intensity for the relaxation and yoga groups. No evidence of a significant difference between groups was observed for any of the other comparisons (music versus standard care; audio-analgesia versus standard care). There was no evidence of significant difference in memory of pain at three-month follow-up for the one study examining this outcome (relaxation versus standard care, one trial, 904 women).

Satisfaction with pain relief: Three of the 11 included trials reported data on satisfaction with pain relief. Two trials reported this as dichotomous data and one trial as continuous data. A significant difference was observed between groups comparing relaxation with standard care (RR $8.00,95 \% \mathrm{Cl} 1.10$ to 58.19 , one trial, 40 women) and between groups comparing yoga with standard care (MD 7.88, 95\% Cl 1.51 to 14.25 , one trial, 66 women), with significantly greater satisfaction reported for women in the relaxation and yoga groups. No significant difference was observed for the other comparison (audio-analgesia versus standard care, one trial, 24 women).
Satisfaction with childbirth experience: Two of the 11 included trials reported data on satisfaction with childbirth experience. A significant difference was observed between groups comparing yoga with standard care (MD 6.34, 95\% Cl 0.26 to 12.42, one trial, 66 women), with greater satisfaction reported for women in the yoga group. No evidence of a significant difference was observed for the other comparison (relaxation versus standard care).

Assisted vaginal birth: Three out of the 11 included trials reported data on assisted vaginal birth. A significant difference was observed between groups comparing relaxation with standard care (RR 0.07, $95 \% \mathrm{Cl} 0.01$ to 0.50 , two trials, 86 women), with fewer women in the relaxation group having assisted vaginal births. No evidence of a significant difference was observed for a cluster trial comparing relaxation with standard care (one trial, 904 women).

Caesarean section: Four out of the 11 included trials reported data on caesarean section. The data could not be combined in a meta-analysis for one of the comparisons because of significant heterogeneity (relaxation versus standard care, three trials [one cluster, two parallel], 990 women). There was no evidence of a significant difference for the other comparison (music versus standard care, one trial, 60 women).

Apgar score less than seven at five minutes: Two out of the 11 included trials reported on Apgar scores. There was no evidence of a significant difference between relaxation versus standard care (one trial, 34 women). In the yoga versus standard care comparison, no babies in either group had an Apgar score less than seven at five minutes.

None of the following outcomes were analysed within the review: sense of control; effect on mother/baby interaction; breastfeeding; adverse effects for women and infants; admission to special care baby unit/neonatal intensive care unit; poor infant outcomes at long-term follow-up; or cost.

\subsection{Comparisons of different types of relaxation techniques}

No studies compared different types of relaxation for pain management in labour.

\subsection{Relaxation versus different interventions}

No studies compared relaxation techniques with other types of interventions to relieve pain in labour.

See Table 18 for all results on relaxation techniques.

\section{Acupuncture or acupressure for pain management in labour (13 studies)}

Thirteen studies with data on 1986 women were included in this review (2391 women in total randomised) (Smith 2011a): eight studies examined acupuncture and four acupressure; the control conditions varied, with women in some studies receiving placebo interventions and in others no intervention. Results for acupuncture and acupressure and for different control conditions were set out separately in the review. One study compared acupuncture versus sterile water injection (128 women) (results for this last study comparing different types of interventions are set out in section 7.3 below). 
7.1. Acupuncture or acupressure versus placebo treatments or standard care (12 studies, 1858 women)

Pain intensity: The tools used to assess pain were not reported in the review. Seven of the 12 included trials reported data on pain intensity as continuous data. A significant difference was observed between groups in a study comparing acupuncture with no treatment (standardised mean difference (SMD) -1.00, 95\% Cl -1.33 to -0.67 , one trial, 163 women) and in studies comparing acupressure with placebo (SMD $-0.55,95 \% \mathrm{Cl}-0.92$ to -0.19 , one trial, 120 women) and with a combined control (SMD -0.42, 95\% $\mathrm{Cl}-0.65$ to -0.18 , two trials, 322 women). Women reported less intense pain in both the acupuncture and acupressure groups for these comparisons. However, this evidence was generally limited to single studies. No evidence of a significant difference between groups was observed for any of the other comparisons (acupuncture versus placebo; acupuncture versus standard care).

Satisfaction with pain relief: The tools used to assess satisfaction with pain relief were not reported in the review. Two of the 12 included trials reported data on satisfaction with pain relief. $A$ significant difference was observed between groups in the study comparing acupuncture with placebo; more women were satisfied with pain relief in the acupuncture group when compared with placebo (RR 2.38, 95\% Cl 1.78 to 3.19, one trial, 150 women). No evidence of a significant difference was observed for the acupuncture versus standard care comparison.

Assisted vaginal birth: Five of the 12 trials reported data on assisted vaginal birth. A significant difference was observed between groups in the studies comparing acupuncture with standard care (RR 0.67, $95 \% \mathrm{Cl} 0.46$ to 0.98 , three trials, 704 women), with significantly fewer women in the acupuncture group having assisted vaginal births. No evidence of a significant difference between groups was observed for any of the other comparisons.

Caesarean section: Eight of the 12 trials reported data on caesarean section. A significant difference was observed between groups in the study comparing acupressure with placebo (RR $0.24,95 \% \mathrm{Cl}$ 0.11 to 0.54 , one trial, 120 women), with significantly fewer women in the acupressure group having caesarean section. There was no evidence of a significant difference between groups for any of the other comparisons.

Apgar score less than seven at five minutes: Five of the 12 trials reported data on Apgar score less than seven at five minutes. There was no evidence of a significant difference between groups in any of the trials.

\subsection{Comparisons of different types of acupuncture}

No studies compared different types of acupuncture.

\subsection{Acupuncture versus sterile water injection (one study, 128 women)}

A significant difference in satisfaction with pain relief was observed between groups in a trial comparing acupuncture with sterile water injections with increased satisfaction reported in the sterile water group (MD $18.60,95 \% \mathrm{Cl} 11.54$ to 25.66 , one trial, 128 women).

There was no evidence of significant differences between groups for assisted vaginal or caesarean birth. Other outcomes were not reported.
None of the following outcomes were analysed within the review: sense of control in labour; satisfaction with childbirth experience; effect on mother/baby interaction; breastfeeding; adverse effects for women and infants; admission to special care baby unit/NICU; poor infant outcomes at long-term follow-up; and cost.

See Table 19 for all results on acupuncture or acupressure

\section{Massage, reflexology and other manual methods for pain management in labour (six studies)}

Six trials were included in this review, with data reporting on five trials and 326 women in the meta-analysis (401 women in total randomised) (Smith 2012). Only studies examining massage were identified. Control conditions varied. Four studies examined massage compared with usual care (225 women). One study examined massage compared with breathing exercises ( 28 women) and one examined massage compared with music. These are reported separately (101 women).

Overall, there was little difference between groups for most of the comparisons examining satisfaction with pain relief, sense of control in labour, assisted vaginal birth, caesarean section or admission to special care baby unit. The only difference observed between groups was for pain intensity.

\subsection{Massage versus standard care (four studies, 225 women)}

In four trials comparing massage with usual care pain intensity during the first stage of labour was reduced in the massage group (SMD -0.82, 95\% Cl -1.17 to $-0.47,225$ women). No evidence of a significant difference between groups for pain intensity was observed during the second or third stages of labour.

\subsection{Comparisons of different manual methods}

No studies compared different types of manual methods for pain management in labour.

\subsection{Massage versus a different intervention (relaxation/ music) (two studies 129 women)}

In one trial comparing massage with music therapy the number of women reporting severe pain was significantly lower in the massage group (RR $0.40,95 \% \mathrm{Cl} 0.18$ to $0.89,101$ women). Data were not in a suitable format for analysis in one trial comparing massage with relaxation (28 women).

None of the following outcomes were analysed within the review: satisfaction with childbirth experience; effect on mother/baby interaction; breastfeeding; adverse effects for women and infants; Apgar score less than seven at five minutes; poor infant outcomes at long-term follow-up; or cost.

See Table 20 for all results relating to massage.

\section{Transcutaneous electrical nerve stimulation (TENS) for pain management in labour (17 studies)}

Seventeen studies involving 1466 women were included in this review (Dowswell 2009): thirteen studies examined TENS applied to the back; two to acupuncture points; and two to the cranium. Fourteen studies compared TENS with placebo or usual care (1256 women); three studies compared TENS as an adjunct to epidural analgesia to epidural alone (200 women); and one study compared TENS versus sterile water injection ( 23 women). This study included 
three arms: TENS, usual care, sterile water. Results for the TENS versus sterile water comparison are set out separately.

Overall, there was little difference between groups for most of the comparisons examining pain intensity, satisfaction with pain relief or assisted vaginal birth. Any differences observed in these outcomes were limited to one or two studies, as outlined below.

\subsection{TENS (to back, cranium or acupuncture points) versus placebo /} standard care (17 studies, 1455 women)

Pain intensity: In two trials (290 women) comparing TENS to acupuncture points versus placebo significantly fewer women in the TENS group reported severe pain during labour compared with controls (RR $0.41,95 \% \mathrm{Cl} 0.31$ to 0.54 ).

Satisfaction with pain relief: In one trial (90 women) comparing TENS to acupuncture points versus control (no pain relief), significantly more women in the TENS group were satisfied with pain relief (RR 4.10, 95\% Cl 1.81 to 9.29).

Assisted vaginal birth: In one trial ( 100 women) comparing TENS to acupuncture points versus placebo, significantly more women in the TENS group had assisted vaginal births (RR $4.50,95 \% \mathrm{Cl} 1.02$ to 19.79).

Other outcomes: There was no evidence of a significant difference between groups for any of the outcomes relating to caesarean section or adverse effects for the infant (fetal distress).

Data were not available in a suitable format for analysis for sense of control in labour, satisfaction with childbirth experience or Apgar score (for the last, all data were provided as means).

\subsection{Different types of TENS}

No studies compared different types of TENS (e.g. TENS to different parts of the body or different intensities).

\subsection{TENS versus sterile water injections (one study, 22 women)}

Results from a single study comparing TENS with sterile water injections reported on mean pain scores but results were difficult to interpret. For other outcomes reported, there were no significant differences between groups.

None of the following outcomes were analysed within the review: effect on mother/baby interaction; breastfeeding; adverse effects for women; admission to NICU or special care baby unit (SCBU); poor infant outcomes at long-term follow-up; or cost.

See Table 21 for all results on TENS.

\section{Pharmacological interventions (six reviews)}

\section{Inhaled analgesia for pain management in labour (26 studies)}

Twenty-six studies randomising 2967 women were included in this review (Klomp 2012). The review presented data for five different comparisons; here we have summarised results under three headings: inhaled analgesia versus placebo/no treatment; versus another (different) inhaled analgesia and versus different methods of pain relief.
1.1. Inhaled analgesia versus placebo (oxygen or compressed air or no treatment) (nine studies, 1495 women)

In the studies comparing inhaled analgesia with placebo or no treatment, nitrous oxide was found to offer better pain relief (average RR $0.06,95 \% \mathrm{Cl} 0.01$ to 0.34 . two studies, 310 women; MD $-3.50,95 \% \mathrm{Cl}-3.75$ to -3.25 , one study, 509 women). However, nitrous oxide was associated with more adverse effects for women such as nausea, vomiting, dizziness and drowsiness (RR 9.05, 95\% $\mathrm{Cl} 1.18$ to 69.32 , two studies, 619 women; RR $43.10,95 \% \mathrm{Cl} 2.63$ to 706.74, one study, 509 women; RR $113.98,95 \% \mathrm{Cl} 7.09$ to 1833.69 , one study, 509 women; RR $77.59,95 \% \mathrm{Cl} 4.80$ to 1254.96 , one study, 509 women) when compared with placebo or no treatment.

1.2. Inhaled analgesia versus different inhaled analgesia (different drugs, strength or delivery system)

1.2.1. Inhaled analgesia versus a different type of inhaled analgesia (nitrous oxide versus flurane derivatives, 14 studies, 752 women)

In the studies comparing different types of inhaled analgesia, mean pain scores were lower with flurane derivatives and women receiving flurane derivatives were more likely to have improved pain relief scores compared with nitrous oxide (average pain score MD $13.87,95 \% \mathrm{Cl} 4.02$ to 23.72 , three studies, 123 women; pain relief score MD $-16.92,95 \% \mathrm{Cl}-27.64$ to -6.20 , two studies, 140 women). Substantial heterogeneity was found in the analyses of pain intensity.

Compared with nitrous oxide, flurane derivatives were associated with less maternal nausea and vomiting (RR $3.30,95 \% \mathrm{Cl} 1.64$ to 6.63 , six studies, 378 women; RR $2.66,95 \% \mathrm{Cl} 1.06$ to 6.70 , four studies, 261 women), but more drowsiness (MD $-12.97,95 \% \mathrm{Cl}$ -22.33 to -3.62 , two studies, 57 women).

\subsubsection{Inhaled analgesia of one strength versus a different strength} ( $50 \%$ nitrous oxide versus $70 \%$ nitrous oxide, two studies, 625 women)

There were no significant differences found for any of the outcomes in the studies comparing one strength versus a different strength of inhaled analgesia.

1.2.3. Inhaled analgesia using one type of delivery system versus a different system (nitrous oxide with or without nasal supplement, methoxyflurane using Penthrane ${ }^{\circledR}$ analgizer versus Cyprane ${ }^{\circledR}$ inhaler, two studies, 75 women)

There were no significant differences found for any of the outcomes in the studies comparing different delivery systems.

\subsection{Inhaled analgesia versus different method of pain relief}

1.3.1 Inhaled analgesia (nitrous oxide) versus TENS (one study, 20 women)

There were no significant differences found for any of the outcomes in the study comparing inhaled analgesia with TENS.

None of the following outcomes were analysed within the review: sense of control; satisfaction with childbirth experience; effect on mother/baby interaction; breastfeeding; admission to special care baby unit; poor infant outcomes at long-term follow-up; or cost.

See Table 22 for all results relating to inhaled analgesia. 


\section{Parenteral opioids for maternal pain management in labour (57 studies)}

Fifty-seven studies involving over 7000 women were included in this large review (Ullman 2010). We have presented results for three main comparisons: first parenteral opioids versus placebo or no treatment; second, comparisons between different opioids; and third, comparisons between parenteral opioids and other methods of pain management in labour. In view of the complexity of this review, within each main comparison we have included subsections, and results for intramuscular (IM) and intravenous (IV) drug administration are set out separately.

Pain intensity was reported in a variety of ways and at different time points across trials. Twenty-eight of the 57 included trials reported data on pain intensity. A total of twenty-three comparisons included data on pain intensity and significant findings were observed in five comparisons.

Satisfaction with pain relief was reported in a variety of ways and at different time points across trials. Only 12 of the 57 included trials reported data on satisfaction with pain relief. A total of nine comparisons included data on satisfaction with pain relief and significant findings were observed in two comparisons.

Satisfaction with childbirth experience was reported in only one of the 57 included trials.

Breastfeeding was reported in only two of the 57 included trials. Definitions of breastfeeding were not provided.

\subsection{Parenteral opioids versus placebo or no treatment (three studies, 226 women)}

2.1.1. IM opioids versus placebo or not treatment (three studies, 226 women)

Only two studies comparing an IM opioid (pethidine) with placebo were included in the review (166 women). In a single study (50 women) more women in the IM pethidine group had a reduction in pain score (defined as a reduction in visual analogue scale score of at least $40 \mathrm{~mm}$ ) compared with the placebo group (RR $25.00,95 \% \mathrm{Cl}$ 1.56 to 400.54 , one study, 50 women). For other outcomes (maternal satisfaction with pain relief, assisted vaginal birth, caesarean section, nausea and vomiting, adverse effects on the baby, and admission to NICU) there was no clear evidence of differences between groups. A single study ( 116 women) reported that women receiving pethidine were more likely to report sleepiness compared with controls (RR 4.67, 95\% Cl 2.43 to 8.95). Other outcomes were not reported in either of these two studies (satisfaction with childbirth experience, breastfeeding, Apgar score less than seven at five minutes, sense of control in labour, effect on mother/baby interaction, poor long-term outcomes in babies and cost).

One study (60 women) compared IM tramadol versus no treatment. This study reported on only one of the overview's outcomes: adverse effects in women (blood loss at delivery). There was no significant evidence of any difference between groups.

\subsubsection{IV opioids versus placebo (no studies)}

None of the studies examining IV opioids (including opioids administered through PCA systems) were compared with placebo or no treatment.

\subsection{Comparisons of different opioids}

2.2.1. IM opioids versus different IM opioids (15 different comparisons, 36 studies)

A broad range of comparisons was examined, with many comparisons confined to evidence from single studies. Studies were carried out over several decades and in several different countries and comparisons included IM pethidine versus meptazinol, diamorphine, tramadol, dihydrocodeine, pentazocine, nalbuphine or morphine.

Pain intensity: This outcome was reported for 13 comparisons examining one type of IM opioid versus another: for 12 comparisons (predominantly measured in single studies comparing pethidine with another opioid) there was no significant evidence of differences between groups. Only one comparison (IM tramadol versus IM pethidine) examined in four studies (243 women) reported a statistically significant difference between groups. More women reported "poor pain relief" in the IM tramadol group compared with the IM pethidine groups (RR $1.56,95 \% \mathrm{Cl} 1.10$ to 2.21).

See Table 23 for all results relating to pain intensity.

Satisfaction with pain relief: This outcome was reported for three comparisons.Pooled results for three studies (365 women) examining IM pentazocine versus IM pethidine found no significant difference between groups. A single study (10 women) comparing PCA (IM) meptazinol and PCA (IM) pethidine also reported no significant difference between groups. In one study fewer women receiving IM nalbuphine group were dissatisfied with pain relief compared with those receiving IM pethidine (RR $0.73,95 \% \mathrm{Cl} 0.55$ to 0.96 , one study, 72 women).

See Table 23 for all results relating to satisfaction with pain relief.

Satisfaction with the childbirth experience: this outcome was not reported in any of the studies included in this comparison. (Table 23)

Breastfeeding: This outcome was reported for only one study: there was no evidence of a significant difference between groups receiving IM meptazinol versus IM pethidine (RR 0.76, 95\% CI 0.17 to 3.30 , one study, 197 women).

See Table 23 for results relating to breastfeeding.

Assisted vaginal delivery: This outcome was reported for six different comparisons (11 studies); there were no significant differences between groups for any of the comparisons (Table 23).

Caesarean section (CS): The number of women undergoing CS was reported for five different comparisons (9 studies); there were no significant differences for any of the comparisons. (Table 23).

Adverse effects: a range of adverse effects was reported, including nausea, vomiting and sleepiness. Measurement of these outcomes varied between studies (some studies reported nausea and vomiting as separate outcomes and some as a combined outcome). The time at which they were measured also varied considerably, which makes results difficult to summarise and interpret. Overall, in many of these trials, women receiving pethidine were more likely to report adverse effects (including nausea and drowsiness) compared with women receiving other opioids. 
Maternal drowsiness or sleepiness was reported for nine different comparisons and for eight of these comparisons there were no significant differences between women receiving different opioids. In studies comparing IM tramadol versus pethidine (five studies, 409 women) fewer women in the tramadol group reported sleepiness (RR $0.57,95 \% \mathrm{Cl} 0.33$ to 0.97 ), but there was variation between the individual studies and substantial heterogeneity was evident $(12=72 \%)$. Results for nausea were reported for six comparisons and there were significant differences between groups for two comparisons: in three studies (391 women) comparing IM pentazocine with IM pethidine, more women in the pethidine group reported nausea (RR $0.46,95 \% \mathrm{Cl} 0.24$ to 0.90 ), and in a single study (301 women) comparing nalbuphine with pethidine, again pethidine was associated with increased rates of nausea (RR $0.62,95 \% \mathrm{Cl} 0.42$ to 0.91 ). Vomiting was recorded for seven comparisons and for four of these there were differences between groups; overall women were less likely to experience vomiting if they received meptazinol (three studies 1589 women), diamorphine (one study, 133 women), nalbuphine (one study, 301 women) and phenazocine (one study 212 women) compared to groups receiving IM pethidine. In three comparisons, nausea and vomiting were reported as a single effect: for two comparisons there was no evidence of significant differences between groups; in a single study (72 women) comparing nalbuphine and pethidine, women receiving pethidine were more likely to report nausea and vomiting. (Table 23)

Adverse effects in infants: a range of adverse effects was reported including neonatal resuscitation, administration of naloxone, respiratory distress and neuro behavioural outcomes. Overall, these outcomes were reported in single studies and results were mainly non-significant. Naloxone administration was reported for five different comparisons and there was no significant evidence of differences between groups receiving pethidine compared with other opioids. Numbers requiring neonatal resuscitation were reported for three comparisons: there was no evidence that infants whose mothers received pethidine, rather than other opioids, were more or less likely to need resuscitation. A single study reported mean scores on a neurological scale at two to four hours post birth (72 babies) and reported lower mean score for babies whose mothers had received nalbuphine. (Table 23). The number of babies admitted to special care was reported for four comparisons (meptazinol, tramadol, diamorphine, nalbuphine, four single studies); in all four cases the control arm received pethidine. There was no evidence of any significant differences for any of the comparisons. (Table 23) Similarly, in four comparisons where Apgar scores at five minutes were examined there was no clear evidence of differences between experimental and control groups (all of which received pethidine). (Table 23)

Sense of control in labour, mother/baby interaction, poor infant outcomes at long-term follow-up and cost were not reported for any comparisons. (Table 23)

\subsubsection{IV opioids versus different IV opioids (12 comparisons, 17 studies)}

Intravenous opioids were compared with other IV opioids in 17 studies although many outcomes were either not reported at all or were reported in single studies. The types of opioids compared in studies included: IV pethidine versus IV fentanyl, butorphanol, pentazocine, remifentanil or nalbuphine, and in some trials opioids other than pethidine were compared with each other: e.g. fentanyl versus alfentanil. In 10 of the studies (seven different comparisons) IVs were administered by staff; in

seven studies (five comparisons) IV drugs were administered through patient-controlled (PCA) systems. In these comparisons, the regimens (lockout times, size of bolus, rate of background infusion) as well as drugs varied between trials. While we have combined methods of IV administration in our summary (e.g. staff or patient-controlled), where results suggest differences between groups we have specified the mode of administration.

Pain intensity: Pain intensity was examined for seven comparisons (eight studies). In five comparisons examining different types of opioid via PCA there was no evidence of differences between groups. In a single study looking at staff-administered IV opioids, the mean pain score in the IV fentanyl group was significantly lower than in the IV pethidine group (MD $-0.20,95 \% \mathrm{Cl}-0.34$ to -0.06 , one study, 105 women). The mean pain relief score (high score = better pain relief) was significantly higher in the IV butorphanol group compared with the IV pethidine (MD $0.67,95 \% \mathrm{Cl} 0.25$ to 1.09 , one study, 80 women) and this finding was supported by data for mean pain scores one hour after drug administration which were significantly lower for women in the butorphanol group (MD -0.60, $95 \% \mathrm{Cl}-1.02$ to -0.18 , one study, 80 women).

See Table 24 for all results relating to pain intensity.

Satisfaction with pain relief: This outcome was poorly reported; only four studies, each describing a different IV comparison provided data on women's views of their pain relief. Three of the four studies reported no significant differences between groups; in a single study, fewer women in the morphine group were satisfied with their pain relief compared with an IV pethidine group (RR 0.87, $95 \% \mathrm{Cl} 0.78$ to 0.98 , one study, 141 women, staff-administered).

See Table 24 for all results relating to satisfaction with pain relief.

Satisfaction with childbirth experience was reported in only one of the 57 included trials. This study found no significant difference in satisfaction with childbirth experience between the PCA remifentanil and PCA pethidine groups (RR 1.10, 95\% $\mathrm{Cl} 0.40$ to 1.74 , one study, 68 women). (Table 24)

Breastfeeding: Results for breastfeeding at hospital discharge were reported in only one study. There was no evidence of a significant difference between groups for PCA pentazocine versus PCA pethidine (RR $1.00,95 \% \mathrm{Cl} 0.85$ to 1.17 , one study, 23 women). (Table 24)

Assisted vaginal birth: this outcome was reported for three different comparisons. In all three, pethidine was compared with other opioids, there was no evidence of differences between groups for any of the comparisons. (Table 24)

Caesarean section: the number of women having CS was reported in nine studies (eight comparisons, four with staff-administered IV drug and four PCA). There was no significant evidence of differences between groups for any of the comparisons. (Table 24)

Adverse effects for women including nausea and vomiting (reported either separately or together) and sleepiness were recorded for several comparisons: for most of these there were no clear differences between women receiving different types of IV opioids. Sleepiness was reported for three comparisons; there 
a difference between groups for only one comparison. In one study with 105 participants, women receiving IV fentanyl were reported to be less sedated than those receiving IV pethidine (RR $0.05,95 \% \mathrm{Cl} 0.00$ to 0.82 ). Nausea and vomiting were reported for five comparisons and again most (4/5) did not find significant differences between groups; in a single study (200 women) fewer women in the IV butorphanol group experienced nausea and/or vomiting compared with those in pethidine group (RR $0.04 .95 \% \mathrm{Cl}$ 0.00 to 0.67 ). (Table 24 )

Adverse effects for infants: The number of babies requiring naloxone or resuscitation was reported for four and two comparisons respectively, and neuro-behavioural scores (at different time points) were reported for four comparisons. None of these comparisons showed significant differences between groups. (Table 24)

The number of babies admitted to special care was only reported in one study (17 women) and there was no evidence of differences between groups. While babies with Apgar scores less than seven at five minutes was reported for five comparisons (six studies) again, none showed statistically significant differences for babies whose mothers had received different opioids.

None of the studies examining IV comparisons reported results for mothers' sense of control in labour, effect on mother-baby interaction, poor infant outcomes at long-term follow-up or cost.

\subsection{Parenteral opioids versus different interventions}

\subsubsection{Parenteral opioids versus a different method of pain} management (TENS) (three comparisons, three studies, 305 women)

Three studies compared parenteral opioids with TENS; each examined a different comparison (IV pethidine, IM pethidine and IM tramadol). For most outcomes there was very little evidence available (data from none or only one of the studies) and there were few statistically significant differences between trial arms.

Pain intensity was reported for two studies (290 women); there was no clear evidence of any difference between groups receiving TENS compared with opioids. Two studies (104 women) reported satisfaction with pain relief and again there was no significant difference between women in the TENS and opioid groups. There was no evidence of differences between groups for assisted vaginal birth or caesarean section (one study, 200 women).

Adverse effects were reported for two studies (290 women); women in the opioid groups were more likely to report drowsiness or nausea and vomiting compared with those in the TENS group, although the $95 \% \mathrm{Cls}$ were very broad for both outcomes (RR 8.96, $95 \% \mathrm{Cl} 1.13$ to 71.07 ; RR $14.06,95 \% \mathrm{Cl} 1.96$ to 100.61 respectively). Fetal distress was reported in one study (200 women) and there was no evidence of differences between groups. (Table 24)

Satisfaction with the childbirth experience, breastfeeding, admission to special care, Apgar score less than seven, sense of control in labour, mother-baby interaction, poor infant outcomes at long-term follow-up and cost were not reported in any of the three studies.
2.3.2. Parenteral opioids versus a different method of pain management (epidural)

Thirty-three studies compared epidural analgesia with IM or IV opioids; findings from these comparisons are discussed below in the section on epidural versus non-epidural or no analgesia in labour.

\section{Non-opioid drugs for pain management in labour (18 studies)}

Eighteen studies randomising 2733 women were included in this review (Othman 2012). The non-opioid drugs included paracetamol, the non-steroidal anti-inflammatory drugs (NSAIDs) (e.g. aspirin, ibuprofen), sedatives (e.g. barbiturates, phenothiazine, and benzodiazepines), anti-spasmodics (e.g. hyoscine), and anti-histamines (e.g. promethazine, hydroxyzine). There were three main comparison groups: 14 studies compared non-opioid drugs with placebo or no treatment (2003 women); three studies compared one type of non-opioid drug with a different non-opioid drug or different doses of the same drug (590 women); and three studies compared non-opioid drugs with opioids (563 women). Three studies had more than two study arms and so data from different arms have been included in more than one comparison group.

Most outcomes were not reported for most of the comparisons. Where outcomes were reported, there was little evidence of any difference for most comparisons. Any differences observed were mainly limited to one or two studies, as outlined below.

\subsection{Non-opioid drugs versus placebo ( 14 studies, 2003 women)}

Sedatives were found to offer better pain relief (MD $-22.00,95 \% \mathrm{Cl}$ -35.86 to -8.14 , one trial, 50 women), better satisfaction with pain relief (RR $1.59,95 \% \mathrm{Cl} 1.15$ to 2.21 , two trials, 204 women) and better satisfaction with childbirth experience (RR $2.16,95 \% \mathrm{Cl} 1.34$ to 3.47 , one trial, 40 women) when compared with placebo or no treatment. Anti-histamines were also found to offer better satisfaction with pain relief (RR $1.80,95 \% \mathrm{Cl} 1.16$ to $2.79,1$ trial 223 women) when compared with placebo or no treatment.

There was no evidence of a significant difference for any of the other comparisons.

\subsection{Non-opioid drug versus a different type or dose of a non- opioid drug (three studies $\mathbf{5 9 0}$ women)}

Women receiving the anti-histamine hydroxyzine were more likely to express satisfaction with pain relief than those receiving the antihistamine promethazine (RR $1.21,95 \% \mathrm{Cl} 1.02$ to 1.43 , one trial, 289 women).There was no evidence of a significant difference for any of the other comparisons reported.

\subsection{Non-opioid drug versus a different type of intervention (opioid) (three studies, 563 women)}

Women receiving non-opioid drugs (NSAIDs or anti-histamines) were less likely to be satisfied with pain relief when compared to women having opioids ( $R R=.50,95 \% \mathrm{Cl} 0.27$ to 0.94 , one trial, 76 women; RR $0.73,95 \% \mathrm{Cl} 0.54$ to 0.98 , one trial, 223 women).

None of the following outcomes were analysed within the review: sense of control in labour; effect on mother/baby interaction; admission to special care baby unit/neonatal intensive care unit; poor infant outcomes at long-term follow-up; or cost. 
See Table 25 for all results relating to non-opioid drugs.

\section{Local anaesthetic nerve block for pain management in labour (12 studies)}

Twelve studies randomising 1549 women were included in this review (Novikova 2012). The review presented data for the following comparisons: local anaesthetic nerve block versus placebo (one study, 200 women); local anaesthetic nerve block versus a different local anaesthetic agent for nerve block (eight studies, 1120 women); local anaesthetic nerve block versus a different intervention (local anaesthetic nerve block versus opioids (two studies, 129 women); and local anaesthetic nerve block versus non-opioids (one study, 100 women)).

For all comparisons results were either not reported or there were no significant differences between groups except for the following results derived from single studies:

\subsection{Local anaesthetic nerve block versus placebo (one study, 200 women)}

Women who received local anaesthetic nerve block were more likely to be satisfied with pain relief compared with women who received placebo (RR $32.31,95 \% \mathrm{Cl} 10.60$ to 98.54 , one study, 198 women). However, mothers and infants of mothers who received local anaesthetic nerve block were more likely to experience adverse effects (mother - giddiness, sweating, tingling of lower limbs; infant - bradycardia) compared with women who received placebo (RR $29.00,95 \% \mathrm{Cl} 1.75$ to 479.61 , one study, 200 women).

\subsection{Local anaesthetic nerve block versus a different type of local anaesthetic nerve block (eight studies, 1120 women)}

In studies comparing one type of local anaesthetic nerve block with a different type of local anaesthetic nerve block, there was no evidence of a significant difference between groups for any outcomes analysed (satisfaction with pain relief; assisted vaginal birth; caesarean section; adverse effects for mother; Apgar score less than seven at five minutes).

4.3. Local anaesthetic nerve block versus a different intervention (opoid or non-opioid drugs) (three studies, 229 women)

Women who received local anaesthetic nerve block were more likely to be satisfied with pain relief compared with women who received intramuscular pethidine ( $\mathrm{RR} 2.52,95 \% \mathrm{Cl} 1.65$ to 3.83 , one study, 109 women). There were no significant differences between local anaesthetic nerve blocks and opioids for assisted vaginal birth or caesarean section (two studies, 129 women) or between local anaesthetic nerve blocks and non-opioids for satisfaction with pain relief or caesarean section (one study, 100 women).

None of the following outcomes were analysed within the review: pain intensity; sense of control; satisfaction with childbirth experience; effect on mother/baby interaction; breastfeeding; admission to special care baby unit; poor infant outcomes at longterm follow-up; or cost.

See Table 26 for all results relating to local anaesthetic nerve block.

\section{Epidural versus non-epidural or no analgesia in labour (38 studies)}

Thirty-eight studies randomising 9658 women were included in this review (Anim-Somuah 2011). The majority of studies, (33 studies), compared epidural analgesia with IM or IV opioids. In five of the included studies epidural was compared to no analgesia during labour.

In this section of the overview we were not able to present separate results for epidural versus no intervention/placebo and epidural versus an alternative method of pain management. This is because, in the original review there was no separate analysis for these different comparisons. Similarly, while most of the women in control groups received IM or IV opioid drugs, there was considerable variation in the agents, doses and modes of administration. However, in the original review results for these different comparisons were analysed together, therefore we have not specified control conditions in the results described below.

Results relating to comparisons between different types of epidural (combined spinal-epidural versus epidural analgesia) were the subject of a different review, and are described in a separate section below.

\subsection{Epidural versus non-epidural or no analgesia in labour ( 38 studies)}

Epidural was found to offer better pain relief (MD -3.36, 95\% Cl -5.41 to -1.31 , three trials, 1166 women), a reduced risk of acidosis in the newborn ( $\mathrm{RR} 0.80,95 \% \mathrm{Cl} 0.68$ to 0.94 , seven trials, 3643 women) and a reduced risk of naloxone administration in the newborn (RR $0.15,95 \% \mathrm{Cl} 0.10$ to $0.23,10$ trials, 2645 women) compared with controls. However, epidural analgesia was associated with an increased risk of assisted vaginal birth (RR $1.42,95 \% \mathrm{Cl} 1.28$ to 1.57 , 23 trials, 7935 women), maternal hypotension (RR $18.23,95 \% \mathrm{Cl}$ 5.09 to 65.35 , eight trials, 2789 women), motor-blockade (RR 31.67, $95 \% \mathrm{Cl} 4.33$ to 231.51 , three trials, 322 women), maternal fever (RR $3.34,95 \% \mathrm{Cl} 2.63$ to 4.23 , six trials, 2741 women), urinary retention (RR $17.05,95 \% \mathrm{Cl} 4.82$ to 60.39 , three trials, 283 women). There was an increased risk of caesarean section for fetal distress (RR 1.43, $95 \% \mathrm{Cl} 1.03$ to $1.97,11$ trials, 4816 women). There was no evidence of a significant difference in the risk of caesarean section overall (RR $1.10,95 \% \mathrm{Cl} 0.97$ to $1.25,27$ trials, 8417 women), long-term back ache (RR $0.96,95 \% \mathrm{Cl} 0.86$ to 1.07 , three trials, 1806 women), Apgar score less than seven at five minutes (RR $0.80,95 \% \mathrm{Cl} 0.54$ to $1.20,18$ trials, 6898 women) and maternal satisfaction with pain relief (RR $1.31,95 \% \mathrm{Cl} 0.84$ to 2.05 , seven trials, 2929 women).

Substantial heterogeneity was found in the analyses of pain intensity and maternal satisfaction. This could not be explained by subgroup or sensitivity analyses.

None of the following outcomes were analysed within the review: effect on mother/baby interaction; breastfeeding; poor infant outcomes at long-term follow-up; or cost.

See Table 27 for all results on epidural.

\section{Combined spinal-epidural versus epidural analgesia in labour (27 studies)}

Twenty-seven studies involving 3303 women were included in this review (Simmons 2012). There was considerable heterogeneity between trials with respect to the drug combinations administered, both intrathecally and epidurally, the timing of subsequent 
dosing after initial analgesia and the method of epidural drug delivery. In the context of categorising the epidural drug dose/ concentration used, the term traditional was used for trials where the epidural local anaesthetic (LA) concentration was the equivalent of bupivacaine $0.25 \%$ or more; lower concentrations were defined as low-dose. In the CSE groups, there were three types of interventions; LA plus opioid, opioid alone, or null CSE where there was a dural puncture with no intrathecal injection of drugs. Using these definitions the comparisons fell into six categories as detailed below:

1. LA plus opioid CSE versus traditional epidural;

2. LA plus opioid CSE versus low-dose epidural;

3. opioid only CSE versus traditional epidural;

4. opioid only CSE versus low-dose epidural;

5. opioid only CSE versus test LA/opioid epidural;

6. null CSE versus traditional epidural.

Analyses were performed on two separate sets of comparisons. The first set involved all combined spinal-epidural (CSE) variants versus traditional epidurals and the second set was all CSE forms versus low-dose epidurals and variants.

In comparison with traditional epidurals, the mean time of onset of effective analgesia was slightly shorter for CSE by approximately three minutes (MD $-2.87,95 \% \mathrm{Cl}-5.07$ to -0.67 , two studies, 129 women) and there were fewer assisted vaginal births (RR $0.80,95 \%$ $\mathrm{Cl} 0.67$ to 0.97 , six studies, 1015 women) and less urinary retention (RR $0.86,95 \% \mathrm{Cl} 0.79$ to 0.95 , one study, 704 women) in women in the CSE group. In comparison with low-dose epidural, again the mean time of onset of effective analgesia was shorter for the CSE group by approximately five minutes (average MD -5.42, 95\% $\mathrm{Cl}-7.26$ to -3.59 , five studies, 461 women) and more women in the CSE group reported effective pain relief at 10 minutes after the first injection (RR $1.94,95 \% \mathrm{Cl} 1.49$ to 2.54 , one study, 101 women). However, more women in the CSE group reported pruritus compared with the low-dose epidural group (average RR 1.80, 95\% Cl 1.22 to 2.65, 11 studies, 959 women). Time to onset of effective analgesia and pruritus showed substantial heterogeneity. There was no evidence of a significant difference between comparison groups for any of the other outcomes analysed.

None of the following outcomes were analysed within the review: satisfaction with pain relief; sense of control in labour; satisfaction with childbirth experience; effect on mother/baby interaction; breastfeeding; poor infant outcomes at long-term follow-up; or cost.

See Table 28 for all results relating to combined spinal-epidural.

\section{Non-Cochrane Systematic reviews}

\section{Non-pharmacological interventions (one review)}

\section{Sterile water injection, (eight studies)}

Eight studies randomising 783 women were included in this review (Hutton 2009). The review included randomised controlled trials comparing intracutaneous or subcutaneous injections of sterile water with a placebo (saline water) or with other non-pharmacological methods of pain relief such as TENS or acupuncture. Five studies used intracutaneous sterile water injections and three used subcutaneous sterile water injections.
The control groups consisted of: acupuncture (one study); TENS and usual care (one study); and isotonic saline injections (six studies). There was overlap in terms of the studies included in this review and the Cochrane reviews on intracutaneous sterile water injection, acupuncture and TENS. However, this non-Cochrane review included additional data on pain intensity and caesarean section, not analysed in the sterile water Cochrane review, and has been included for this reason.

In comparison with placebo or other intervention groups, there was a significant reduction in VAS pain score in the sterile water group at three different time-points: 10 to 30 minutes following administration of the intervention (WMD $-26.04 \mathrm{~mm}, 95 \% \mathrm{Cl}-34.14$ to -17.94 , four studies, 289 women); at 45 to 60 minutes (WMD $-36.27 \mathrm{~mm}, 95 \% \mathrm{Cl}-50.80$ to -21.74 , five studies, 542 women); and at 90 to 120 minutes following administration of the intervention (WMD -27.74 mm, 95\% Cl -39.03 to -16.45 , five studies, 488 women). However, substantial heterogeneity was evident within each of these analyses of pain scores. All analyses of pain score used random-effects. A significant reduction was also observed in caesarean sections in a meta-analysis of all eight studies comparing any sterile water injection with placebo (normal saline injection) or with other non-pharmacological interventions such as TENS or acupuncture (RR $0.51,95 \% \mathrm{Cl} 0.30$ to $0.87, \mathrm{l}^{2}=0 \%$ ).

None of the following outcomes were analysed within the review: satisfaction with pain relief; sense of control in labour; satisfaction with childbirth experience; effect on mother/baby interaction; breastfeeding; assisted vaginal birth; adverse effects for mother and baby; admission to special care baby unit; Apgar score less than seven at five minutes; poor infant outcomes at long-term follow-up; and cost.

See Table 29 for all results relating to sterile water injection.

\section{Pharmacological interventions (two reviews) \\ Epidural ropivacaine versus bupivacaine, (23 studies)}

Seven studies randomising 2074 women were included in this review (Halpern 2003a). The review included randomised controlled trials comparing epidural ropivacaine with epidural bupivacaine. There was no evidence of any statistically significant difference between drugs in the incidence of any obstetric or neonatal outcomes assessed (pain intensity; satisfaction with pain relief; assisted vaginal birth; caesarean section; hypotension; nausea or vomiting; Apgar score less than seven at five minutes).

None of the following outcomes were reported within the review: sense of control in labour; satisfaction with childbirth experience; effect on mother/baby interaction; breastfeeding; admission to special care baby unit; poor infant outcomes at long-term followup; and cost.

See Table 30 for all results relating to ropivacaine versus bupivacaine.

\section{Intrathecal opioids, (24 studies)}

Twenty-four studies randomising 3513 women were included in this review (Mardirosoff 2002). The review included randomised controlled trials comparing any analgesia using intrathecal opioid with any non-intrathecal regimen. Three intrathecal opioids were included (sufentanil, fentanyl, morphine), with or without various 
doses of intrathecal or epidural bupivacaine. The controls consisted of different doses of epidural or intrathecal bupivacaine, epidural lidocaine, combinations of epidural bupivacaine and different doses of epidural sufentanil or fentanyl and intravenous sufentanil.

A significant increase in the risk of fetal bradycardia was observed for women in the intrathecal opioid group compared to the control group (RR 29.6, 95\% 13.6 to 64.6, 11 studies, 855 women) and for maternal pruritus in the intrathecal groups compared with controls who had not received opioids (RR $29.6,95 \% \mathrm{Cl} 13.6$ to 64.6 , 11 studies, 855 women). There was no evidence of a significant difference for maternal pruritus when intrathecal opioids were compared with IV or IM opioid controls (average RR 1.71, 95\% CI 0.97 to 3.02). There was no evidence of any significant differences between groups for any other outcomes examined (assisted vaginal birth; caesarean section; fetal heart rate abnormalities; Apgar score less than seven at five minutes).

None of the following outcomes were analysed within the review: pain intensity; satisfaction with pain relief; sense of control in labour; satisfaction with childbirth experience; effect on mother/ baby interaction; breastfeeding; poor infant outcomes at long-term follow-up; and cost.

See Table 31 for all results relating to intrathecal opioids.

\section{DISCUSSION}

\section{Summary of main results}

The 15 Cochrane systematic reviews included a total of 257 trials. The non-Cochrane systematic reviews included a total of 55 trials. Most of the comparisons within each individual review were within treatment comparisons e.g. one type of inhaled analgesia compared to a different type of inhaled analgesia. There was a paucity of placebo controlled trials with less than half, $41 \%$ (105), of all included studies comparing the intervention with placebo or no treatment. Similarly, only $18 \%$ (47) of all included studies compared an intervention with a different type of intervention according to the hierarchy of interventions. Most of these studies comparing one form of intervention with another were confined to one review and examined epidural versus parenteral opioids. In other reviews, head-to-head comparisons between different types of interventions were rare, and results were largely derived from a small number of studies (e.g. TENS versus sterile water, one study; inhaled analgesia versus TENS, one study; opioids versus TENS, three studies; non-opioids versus opioids, three studies; local anaesthetic nerve blocks versus opioids, two studies; and local anaesthetic nerve blocks versus non-opioids, one study) (Figure 2).

\section{Based on the following comparisons:}

1. intervention versus placebo or standard care;

2. different forms of the same intervention (e.g. one opioid versus another opioid);

3. one type of intervention versus a different type of intervention (e.g. TENS versus opioid).

We have categorised interventions into " What works," "What may work", and "Insufficient evidence to make a judgement", as outlined.

\section{WHAT WORKS}

There is considerable evidence to suggest that epidurals (including combined spinal epidurals (CSE)), and more limited evidence to suggest that inhaled analgesia are effective for managing pain in labour, but these interventions are not without adverse effects.

\section{Epidural}

There was a reduction in mean pain scores for women in the epidural group when measured during the whole of labour (three studies, 1166 women) and during the first (four trials, 589 women) and second stages of labour (three trials, 559 women) in comparison with non-epidural (parenteral opioids) or no analgesia. However, considerable heterogeneity was observed for these analyses. There was no significant difference observed for any of the other effectiveness outcomes examined (patient satisfaction with pain relief; sense of control in labour; satisfaction with childbirth experience).

More women in the epidural group had assisted vaginal births (23 trials, 7935 women) and experienced adverse effects such as hypotension (eight trials, 2789 women), motor blockade (three trials, 322 women), fever (six trials, 2741 women) or urinary retention (three trials, 283 women) in comparison with nonepidural (parenteral opioids) or no analgesia. More women in the epidural group had caesarean sections for fetal distress (11 trials, 4816 women) in comparison with non-epidural (parenteral opioids) or no analgesia, although there was no evidence of a significant difference between groups in the overall caesarean section rate (seven trials, 8417 women). The risk of acidosis and the requirement for naloxone was lower in infants in the epidural group compared with the non-epidural group (seven trials, 3643 women; one trial, 2645 women). There was no evidence of a significant difference between groups in admissions to special care baby unit (seven trials, 3125 women) or Apgar scores (18 trials, 6898 women).

\section{Combined spinal epidural (CSE)}

In comparison to traditional epidural, the mean time of onset of effective analgesia was slightly shorter for CSE by approximately three minutes (two trials, 129 women). In comparison to low-dose epidural, again the mean time of onset of effective analgesia was shorter for the CSE group by approximately five minutes (five trials, 461 women). However, considerable heterogeneity was observed for the CSE versus low dose comparison. More women in the CSE group reported effective pain relief at 10 minutes after the first injection in comparison with the low dose epidural (one trial, 101 women), but there was no significant difference observed between CSE and low dose epidural for satisfaction with pain relief (seven trials, 520 women). Other effectiveness outcomes (sense of control; satisfaction with childbirth experience) were not reported.

CSE was associated with fewer assisted vaginal births in comparison to traditional epidural (six trials, 1015 women), but there was no evidence of a significant difference in assisted vaginal birth between CSE and low dose epidural (11 trials, 1612 women). There was no evidence of a significant difference in Caesarean section for any of the comparisons: CSE versus traditional epidural (six trials, 1015 women); or CSE versus low dose epidural (15 trials, 1960 women). No differences in adverse effects for women were observed for the following comparisons: post dural puncture; known dural tap; blood patch; nausea and vomiting; hypotension; headache; and sedation. In contrast, fewer women in the CSE group experienced urinary retention compared with traditional 
epidural (one trial, 704 women), but more women in the CSE group experienced pruritus in comparison with the low dose epidural group (11 trials, 959 women). There was no evidence of a significant difference in admissions to special care baby unit or Apgar scores for any of the comparisons: CSE versus traditional epidural; CSE versus low dose epidural.

\section{Inhaled analgesia}

In comparison with placebo, fewer women in the inhaled analgesia group experienced severe or extreme pain during the first stage of labour (two trials, 310 women) and the mean pain score in one large study (one trial, 509 women) was lower. There was insufficient evidence on efficacy in the comparison of inhaled analgesia with TENS, with non-significant findings for pain intensity (one trial, 19 women). More women experienced vomiting (two studies, 619 women), nausea (one study, 509 women), dizziness (one study, 509 women) and drowsiness (one study, 509 women) with inhaled analgesia when compared to placebo. There were no data on safety outcomes for the comparison of inhaled analgesia with TENS.

\section{WHAT MAY WORK}

There is some evidence to suggest that immersion in water relaxation, acupuncture, massage, local anaesthetic nerve blocks and non-opioid drugs may assist in managing pain in labour. Few adverse effects are reported. Evidence was mainly limited to individual trials.

\section{Immersion}

Individual trials comparing immersion in water during the first stage of labour with no immersion or standard care suggested a reduction in pain (one trial, 120 women) and an increase in satisfaction with childbirth experience for those in the immersion group during the second stage of labour (one trial, 117 women). However, in other studies using different pain intensity measures or measurement at different time-points, there was no evidence of a significant difference between groups for pain intensity (one trials, 141 women) and satisfaction with the childbirth experience (one trial, 60 women). Mean blood pressure was lower during the first stage of labour in women in the immersion group in one trial (120 women), but there was no evidence of a significant difference between groups for any other of the safety outcomes.

\section{Relaxation}

A reduction in pain (two trials, 74 women; one trial 66 women), greater satisfaction with pain relief (one trial, 40 women; one trial 66 women) and childbirth (one trial, 66 women) were observed in individual trials of relaxation methods (breathing, yoga) in comparison with standard care. However, for other comparisons of relaxation methods (music; audio-analgesia), there was no evidence of a significant difference for these same outcomes. Fewer assisted vaginal births were observed in two trials comparing relaxation with standard care (two trials, 86 women), but no evidence of a significant difference was observed in one cluster trial of the same comparison (one trial, 904 women).

\section{Acupuncture}

A reduction in pain (one trial, 120 women) and greater satisfaction with pain relief (one trial, 150 women) was observed in individual trials of acupuncture or acupressure in comparison with no treatment or placebo. In one trial comparing acupuncture with sterile water injection, more women in the sterile water group were satisfied with their pain relief (one trial, 128 women). There was no evidence of a significant difference for pain and satisfaction with pain relief for other comparisons of acupuncture with placebo or standard care. Fewer assisted vaginal births (three trials, 704 women) and caesarean sections (one trial, 120 women) were observed in comparisons of acupuncture and acupressure with standard care or placebo. In other comparisons of acupuncture or acupressure, there was no evidence of a significant difference in assisted vaginal births (one trial, 208 women; one trial 163 women; one trial, 222 women) or caesarean sections (three trials, 448 women; two trials, 506 women).

\section{Massage}

In four trials comparing massage with standard care, pain intensity during the first stage of labour was reduced in the massage group during the first stage of labour (four trials, 225 women). No evidence of a significant difference between groups for pain intensity was observed during the second (two trials, 124 women) or third stages of labour (two trials, 122 women). In one trial comparing massage with music therapy, the number of women reporting severe pain was lower in the massage group (one trial, 101 women). There was no evidence of a significant difference in assisted vaginal births (two trials, 105 women), caesarean sections (two trials, 105 women) or admission to special care baby unit (one trial, 44 women) in trials comparing massage with standard care. There were no data on safety outcomes in trials comparing massage with relaxation or music therapy.

\section{Local anaesthetic nerve blocks}

More women in the local anaesthetic nerve block group (PCB 1\% lidocaine) were satisfied with pain relief (degree of pain relief rated as excellent/complete) compared with the placebo group, (one trial, 198 women). There were no data on pain intensity, sense of control and satisfaction with the childbirth experience for this comparison. Women who received local anaesthetic nerve blocks were more likely to be satisfied with pain relief compared with women who received intramuscular pethidine (one trial, 109 women), but there was no evidence of a significant difference between local anaesthetic nerve blocks and (IM promethazine) for satisfaction with pain relief (one trial, 20 women). Local anaesthetic nerve blocks (using lidocaine) were associated with more adverse effects for women and infants (women - sweating, giddiness, tingling of lower limbs; infants - bradycardia; one trial, 200 women) in comparison with placebo. There was no evidence of a significant difference in assisted vaginal births (two trials, 129 women) or caesarean sections (two trials, 129 women) between local anaesthetic nerve blocks and opioids (IM pethidine or PCA fentanyl).

\section{Non-opioid drugs}

There was insufficient evidence on the effectiveness and safety of most non-opioid drugs. However, evidence from single trials suggests that some drugs may work. Non-opioid drugs (sedatives) were found to offer better pain relief (one trial, 50 women), better satisfaction with pain relief (sedatives and anti-histamines: two trials, 204 women; one trial, 223 women) and better satisfaction with the childbirth experience (one trial, 40 women) when compared with placebo. There was no evidence of a significant 
difference for any of the other comparisons of non-opioids (antihistamines) for pain intensity and satisfaction with pain relief. Women having non-opioid drugs (NSAIDs or anti-histamines) were less likely to be satisfied with pain relief when compared with women having opioids (one trial, 76 women).There was little data and no evidence of a significant difference for any of the comparisons of non-opioids for safety outcomes.

\section{INSUFFICIENT EVIDENCE TO MAKE A JUDGEMENT}

There is insufficient evidence to make a judgement on whether or not hypnosis, biofeedback, sterile water injection, aromatherapy, TENS, and parenteral opioids, are more effective than placebo or other interventions for pain management in labour.

\section{Hypnosis}

There was no evidence of a significant difference between hypnosis and either no hypnosis/standard care or a different type of hypnosis for all effectiveness outcomes analysed (satisfaction with pain relief; satisfaction with childbirth experience). There was no evidence of a significant difference in: breastfeeding (one trial, 266 women); assisted vaginal births (three trials, 414 women); caesarean sections (three trials, 867 women); adverse effects for women and infants (one trial, 305 women); admissions to the neonatal intensive care unit (two trials, 345 women); or Apgar score less than seven at five minutes (one trial, 305 women).

\section{Biofeedback}

There was no evidence of a significant difference between biofeedback and standard care for assisted vaginal birth (two trials, 103 women) or caesarean section (two trials, 103 women). No other data on effectiveness or safety outcomes were reported.

\section{Sterile water injection}

In the sterile water versus placebo comparison, data were either not in a suitable format for analysis (pain intensity) or were not reported (satisfaction with pain relief; sense of control in labour; satisfaction with childbirth experience). In the non-Cochrane review, in comparison with placebo or other intervention groups, there was a significant reduction in VAS pain score in the sterile water group at three different time-points. However, substantial heterogeneity was evident within each of these analyses of pain score.

There was no evidence of a significant difference in assisted vaginal births (six trials, 666 women) or caesarean section (seven trials, 766 women) between the sterile water and placebo groups. In the non-Cochrane review, a significant reduction was observed in Caesarean sections in the sterile water group. However, this review included sterile water injection versus any comparison (placebo, TENS, acupuncture) (eight trials, 828 women).

\section{Aromatherapy}

There were no data on any effectiveness outcomes. There was no evidence of a significant difference between groups (aromatherapy versus standard care) in assisted vaginal births, caesarean sections or admission to special care baby unit (one trial, 513 women).

\section{TENS}

In two trials comparing TENS to acupuncture points with placebo, fewer women in the TENS group reported severe pain during labour (two trials, 290 women). No significant differences were observed for pain in two other trials comparing TENS to the back with placebo or usual care (two trials, 299 women). In one trial comparing TENS to acupuncture points with standard care, more women in the TENS group were satisfied with their pain relief (one trial, 90 women) but in five trials comparing TENS to the back with placebo or standard care, there was no difference between groups for satisfaction with pain relief (five trials, 452 women). There were no significant differences observed between groups for assisted vaginal births, caesarean sections or adverse effects for infants.

\section{Parenteral opioids}

More women in the IM pethidine group had a reduction in pain score compared with the placebo group (one trial, 50 women). In studies comparing parenteral opioids with a different intervention, TENS, pain intensity was reported for two studies (290 women); there was no clear evidence of any difference between groups receiving TENS compared with opioids. Two studies (104 women) reported satisfaction with pain relief, and again there was no significant difference between women in the TENS and opioid groups.

There was no clear evidence of differences between groups (parenteral opioids versus placebo) for other outcomes (assisted vaginal birth, caesarean section, nausea and vomiting, adverse effects on the baby, and admission to NICU). A single study (116 women) reported that women receiving pethidine were more likely to report sleepiness compared with controls. Other safety outcomes were not reported.

In studies comparing parenteral opioids with TENS, there was no evidence of differences between groups for assisted vaginal birth or caesarean section (one trial, 200 women). Adverse effects were reported for two studies ( 290 women); women in the opioid group were more likely to report drowsiness or nausea and vomiting compared with those in the TENS group although 95\% CIs were very broad for both outcomes. Fetal distress was reported in one study (200 women) and there was no evidence of differences between groups. Other safety outcomes were not reported.

\section{Overall completeness and applicability of evidence}

The overview has included 18 reviews focusing on 15 different methods of pain relief in labour and most reviews considered several different comparisons. The overview has focused on methods of pain management that are explicitly intended to relieve pain and has not included more broadly based interventions.

There remain gaps in the research evidence and some of the evidence presented in this overview has serious limitations. Most outcome data included in meta-analyses is drawn from only one or two trials within each of the reviews. There are exceptions to this, for example in the epidural and combined spinal epidural reviews a larger number of trials contribute data, but even within these reviews the variability in the results in individual trials (and the high heterogeneity) means that results may still be difficult to interpret. Therefore, it may be difficult, under these circumstances, to generalise findings to other settings.

Most reviews included comparisons with placebo or between different types of the same intervention. However, at the outset we had hoped that we would be able to compare directly different 
methods of pain relief in terms of efficacy and safety; this proved problematic. Very few of the reviews included any direct comparisons with other interventions (Figure 2). Where different methods were compared (e.g. inhaled analgesia versus TENS), results for particular outcomes were mainly confined to evidence from single studies. Thus, with the exception of the comparisons between epidural versus parenteral opioids, we were not able to draw any conclusions from direct comparisons regarding the relative effectiveness and drawbacks of different ways of managing pain in labour.

We considered making indirect comparisons in order to address questions concerning the relative performance of different methods of pain relief. This would have involved using statistical methods to examine different interventions, each compared with the same comparator (e.g. placebo), but which had not been directly compared with each other. However, such methods can only be used meaningfully if the populations recruited to the trials, and the way outcomes are measured, are broadly similar. Therefore, we decided that indirect comparisons would not be appropriate, due to differences between reviews in terms of contextual and other factors. For example, women in trials of IM opiates were recruited over a long period (some trials dating back to the 1930s) while other methods of pain management have only been introduced into obstetric and midwifery practice (and been studied in trials) in the last twenty years. Improved hospital facilities, changes in custom and practice (for example where women give birth and whether or not they have support from partners or family) and the options for pain relief available to women are likely to have changed women's expectations about pain relief and their experience of childbirth. The way pain has been measured has also altered considerably. Few early trials used VASs or validated pain scales, and in some older studies, women were not asked to rate their pain at all. Comparing like with like in terms of populations and outcomes did not seem possible. This meant that we were not able to provide a simple answer to the question about which methods are most effective, safe and acceptable to women.

Many of our prespecified outcomes were not reported in the contributing studies/review. In Table 32 and Table 33 we have provided a summary of outcomes reported within each of the reviews. Sense of control in labour and breastfeeding were very rarely reported within included trials in individual reviews. Effect on mother/baby interaction, poor infant outcomes at long-term follow-up and cost were never reported. As these are all important outcomes to women or to providers of healthcare services, it is surprising that they do not receive more research attention.

The very many ways outcomes were defined and measured in trials led to problems in interpreting findings. It was not always clear which tool was used to measure pain or which component of the McGill pain questionnaire (three components - visual analogue scale, verbal response scale, present pain intensity scale). Some trials just reported "pain scores" and presented data as mean scores. The majority of studies used visual analogue scales, but it was not always clear how the extremes of the scales were marked and whether the line was subdivided in any way. Many trials only presented results graphically or presented median and interquartile range. Some studies measured pain and pain relief as dichotomous outcomes; but again there was no consistency in the meaning of the outcome: a score could mean no pain (or complete relief) or manageable pain (some relief). There was similar variation for satisfaction with pain relief. Many trials also reported mean or median values for Apgar score. Accordingly, a lot of data could not be analysed according to our criteria.

It was sometimes difficult to interpret the outcome data within reviews, as some reviews used different terminology to that specified within the generic protocol. This was probably due to the variation of reporting of outcomes in the individual trials. For example, in some trials, pain was reported as "memory of pain", "degree of pain relief", "perception of pain relief" and experience of childbirth as "difficulty of labour." This made it difficult to map outcome data to outcomes from the core list.

Interpreting findings from individual reviews and summarising findings within this overview were complicated by the variation in both the experimental and control conditions examined within reviews. In this overview, interventions in individual studies have not been described in detail; settings, exact content and the intensity or dose of interventions may have varied; some interventions may have required specialised staff (e.g. acupuncture) and the training and skills of operators are likely to have varied over time and locations. Control conditions are frequently not described at all in trials; understanding what the terms "routine care", "standard care" or "no intervention" mean in studies carried out over a period of more than fifty years in countries across the globe is particularly difficult. Within this overview we have not been able to provide details of participants, interventions (and control conditions) in individual trials and we have given only limited information about the way outcomes were measured in studies and reviews; we would therefore encourage readers to consult the individual reviews to obtain more information on these important factors.

A further difficulty in interpreting evidence and applying findings from the overview to clinical situations relates to gaps in the evidence concerning co-interventions and to the general lack of consistency and clear information about when in labour interventions were implemented and outcomes measured. In practice, women in labour may opt for more than one method of pain relief and may use different interventions simultaneously (e.g. inhaled analgesia and parenteral opioids) or consecutively (e.g. TENS and then epidural). Single interventions may be only partially effective and methods that may provide adequate relief during the early stages of labour may not do so as labour progresses. Without evidence about pain relief at different stages in labour it remains difficult to make recommendations to women when pain intensity is likely to vary considerably as labour progresses.

No subgroup analyses were conducted on the pre-specified subgroups (spontaneous labour versus induced labour; primiparous versus multiparous; term versus preterm birth; continuous support in labour versus no continuous support). This was due to the low number of studies contributing data within the meta-analyses and also due to a lack of clear subgroup data provided within the reviews e.g. no data on continuous support in labour was reported upon within the reviews.

\section{Quality of the evidence}

All fifteen of the Cochrane systematic reviews used the domainbased evaluation for assessments of risk of bias as outlined in Chapter 8 of the Cochrane Handbook for Systematic Reviews of 
Interventions (Higgins 2011). None of the included systematic reviews used the GRADE approach to assess the quality of evidence across studies for each important outcome. We have therefore based our assessments of the quality on the assessments reported in the 'Risk of bias' tables in the included systematic reviews. The risk of bias of included trials within the Cochrane reviews was variable, but generally considered to be high.

The proportion of studies assessed as being at low risk of bias in the non-pharmacological and pharmacological reviews are summarised in Table 6, Table 7. A higher proportion of studies in the non-pharmacological reviews were considered to be at low risk of bias for the domains of sequence generation (54\% versus $31 \%$ ) and allocation concealment ( $40 \%$ versus $27 \%$ ). A higher proportion of studies in the pharmacological reviews were considered to be at low risk of bias for blinding of participants, personnel and outcome assessors (38\%, 38\%, 25\% versus 20\%, 18\%, 31\%). Assessment of blinding was not always conducted for all three groups e.g. some reviews just assessed blinding as a single entity e.g. aromatherapy review, combined spinal epidural review. A higher proportion of studies in the pharmacological reviews were considered to be at low risk of bias for selective outcome reporting and other potential threats to validity ( $49 \%, 41 \%$ versus $26 \%, 39 \%)$. An equivalent number of studies were assessed as low risk of bias for the domain of incomplete outcome assessment.

All three non-Cochrane systematic reviews used the Jadad scale (Jadad 1996) or a modified version to assess the methodological quality of included studies. The Cochrane Collaboration advises against the use of such scales: these are thought to be a poor way of assessing risk of bias, as they omit important considerations and are largely concerned with reporting rather than conduct. The Jadad scale is based on assessment of three items: method of randomisation; blinding; and withdrawals and dropouts. This scale has a maximum of five points, with a score of five indicating the best possible score. In the epidural ropivacaine versus bupivacaine review (Halpern 2003a), 17 out of 23 studies (74\%) were assessed as being high quality (with a score of three or more). In the intrathecal opioids review (Mardirosoff 2002), the median quality score of the 24 included studies was reported to be 3.5 (range 1 to 5). The sterile water injection review used an initial version of the Jadad scale (Jadad 1996), which was based on eleven items. The maximum possible score for this initial instrument is 13 points. In the sterile water injection review (Hutton 2009), seven out of eight studies $(88 \%)$ were assessed as being high quality (with a score of 10 or more).

The methods used in Cochrane reviews were assessed using the AMSTAR rating scale described above. As all Cochrane reviews followed a generic protocol specifying methods, scores were high for all reviews. For the non-Cochrane reviews scores were less high; this was generally because some aspects of the review process may not have been explicit in the published reviews. Findings regarding the quality of contributing reviews are set out in Table 10, Table 11, Table 12.

\section{Potential biases in the overview process}

We are aware that there was a risk of introducing bias at all stages in the overview process. We took a number of steps to try to reduce this. For non-Cochrane reviews, evidence of using systematic methods was part of our criteria for selecting reviews for inclusion. For Cochrane reviews, all reviews used a protocol that aimed to minimise bias. For the overview, two review authors independently assessed eligibility for inclusion of reviews, and carried out data extraction. Data checks were carried out by a third author.

One potential source of bias relates to us, being the authors of some of the included Cochrane reviews. One advantage of this is that we are well informed of the weaknesses and strengths on which these reviews build. No 'Summary findings' tables were produced within individual reviews, which limits our ability to assess overall quality of the evidence against each of our core outcomes.

There is also a risk of bias arising from the fact that not all of the contributing reviews had been completed at the time of preparing the overview. Four of the 15 included Cochrane reviews were in draft form; while all had been submitted for publication in The Cochrane Library, they had not been revised after peer review, or approved for publication. Therefore, the findings we have reported in this overview for these four reviews (examining hypnosis (Madden 2012), inhaled analgesia (Klomp 2012), non-opioid drugs (Othman 2012) and combined spinal epidural (Simmons 2012)) are based on the draft reviews submitted by the authors for editorial consideration. We plan to check all results and make any necessary corrections in the first update of the overview.

However, it is not possible to eliminate all risk of bias; evidence synthesis is not an exact science and involves judgement. We would encourage readers to consider all of the additional tables to assist them in interpreting results.

\section{Agreements and disagreements with other studies or reviews}

Many of the non-Cochrane reviews that we excluded from this overview focused on the same methods of pain management as the included Cochrane reviews. For the reviews that included randomised controlled trials, there was considerable overlap in the studies contributing evidence, despite some differences in search strategies and selection criteria. In view of this overlap, for our primary outcomes, it is therefore not surprising that the overall findings of included and excluded reviews were generally in agreement.

Findings for hypnosis from the Cochrane review were inconclusive. This is in line with an earlier review of non-pharmacological interventions for pain relief in labour, Simkin 2004a, that concluded, at that time, there was insufficient evidence to draw conclusions about the effectiveness of hypnosis and findings. The included Cochrane review focusing on sterile water injection suggested that there was some limited evidence that this method offers some relief from pain. A review by Fogarty 2008 concluded that sterile water injection had a beneficial effect on pain and the included non-Cochrane review Hutton 2009 concurs with this. Immersion in water was the subject of several excluded reviews (Benfield 2002; Huntley 2004; Simkin 2002; Simkin 2004a). While Simkin 2002 suggests that immersion in water reduces labour pain, the evidence was drawn from non-randomised studies. The overall conclusion of this and the other reviews was that there were few differences between intervention and control groups for most outcomes and for many outcomes there was insufficient evidence from trials to draw any firm conclusions. 
For relaxation techniques, massage and reflexology, again other reviews agree that more evidence is needed (Huntley 2004; Simkin 2002; Simkin 2004a).

The value of acupuncture for pain relief in labour was examined by Cho 2010, Lee 2004 and Smith 2009. The results of these reviews are consistent with the findings in the included Cochrane review; while evidence from single studies suggests that acupuncture reduces pain scores and results in increased satisfaction with pain relief compared with controls, overall the evidence is mixed, and for many outcomes there is insufficient evidence to draw firm conclusions. Cho 2010 also points to the mixed methodological quality of trials examining this intervention and concludes that the current evidence from trials does not support the use of acupuncture in labour.

An early review carried out by Carroll 1997 concluded that the evidence on TENS was weak. While the amount of evidence on TENS has increased, the evidence remains inconclusive. Although many women seem to like TENS, it is not clear whether it is an effective means of relieving pain in labour.

The conclusion of the included Cochrane review on inhaled analgesia is reflected in the findings of another review. Rosen 2002a suggests that inhaled analgesia offers safe, reasonably effective pain relief for many women. The review by Klomp 2012, included in this overview, however, highlights some of the adverse effects (such as nausea and drowsiness) associated with some types of inhaled analgesia.

Drawing conclusions regarding the effectiveness and safety of parenteral opioids has been hampered by the large number of studies examining different types, doses and methods of administration of opioids. Despite the large number of studies focusing on this widely used form of pain relief, very few studies compare the effects and adverse effects of different drugs. A decade ago Bricker 2002 indicated the paucity of evidence regarding the relative effects and the adverse effects of different opioids and called for more research directly comparing the most frequently used drugs. Many of the questions raised by that review remain unanswered.

A relatively large number of excluded reviews focused on the use of epidurals. Again findings are broadly similar to those in this overview. In earlier studies there was some concern that epidurals increased the risk of interventions in labour; in particular there was a suggestion that rates of CS were higher in women who received epidurals. However, in some reviews at least part of the evidence on labour interventions was drawn from observational studies rather than trials (Lieberman 2002; Morton 1994; Thorp 1996; Zhang 1999). In the current overview, while there was no strong evidence that, compared with controls, women receiving epidural analgesia were at increased risk of CS overall, the rate of CS for fetal distress was greater, as was assisted vaginal birth. While there is now good evidence that epidural offers good pain relief, there is also evidence that some women experience adverse effects, in addition to any risks associated with instrumental birth. Other non-Cochrane reviews shed more evidence on different types of epidural and different drug regimens (including PCEA) (e.g. Halpern 2009).

An outcome which was considered in very few trials or in included reviews, was the cost of interventions to healthcare providers, including both the direct costs of interventions and the costs of treating adverse effects and complications in women associated with different types of labour analgesia. Huang 2002 examined the costs associated with epidural analgesia; overall however, we found little evidence on this important process outcome.

A gap in the evidence in this overview relates to broader interventions that may increase women's comfort and sense of control in labour, which in turn may increase women's sense of well being, their ability to maintain control, and which may mitigate the experience of pain. Hodnett 2002, in a review of factors which affect women's experience of giving birth and their satisfaction with their labour and birth, pointed to several factors which are important to women. These include the amount of support from caregivers, the quality of women's relationships with caregivers and their sense of involvement in decision-making. Designing interventions which operationalise these factors in clinical trials is challenging, but evidence from surveys and qualitative research has demonstrated their importance to women, and underlines the need for high quality obstetric and midwifery care, where women are consulted and their views are respected.

Within the scope of this overview, we have been unable to evaluate the impact on pain in labour of broadly based interventions such as continuity of caregiver, the value of childbirth preparation classes, mobility in labour and the impact of different types of physical environment for the birth (including home birth) on pain in labour. Other Cochrane reviews focus on these important topics, (Hatem 2008; Hodnett 2007).

\section{AUTHORS' CONCLUSIONS}

\section{Implications for practice}

Most methods of non-pharmacological pain management are noninvasive and appear to be safe for the mother and baby, however their effectiveness is unclear due to limited high quality evidence.

There is more evidence to support the efficacy of pharmacological methods but these also have more known adverse effects. Thus, epidural analgesia provides effective pain relief but at the cost of increased medical intervention including increased incidence of instrumental vaginal birth.

It remains important to tailor methods used to an individual woman's wishes, needs and individual circumstances (this may include judgements about anticipated duration of labour, condition of the infant, or whether the labour is augmented).

\section{Implications for research}

A major challenge in compiling this overview, and the individual systematic reviews on which it is based, has been the variation in use of different outcome measures in different trials, particularly in assessment of pain and in its relief. This has made it difficult to pool results from otherwise similar studies, and to derive conclusions from the totality of evidence.

Other important outcomes have simply not been assessed in trials; thus, despite concerns for 30 years or more about the effects of maternal opiate administration during labour on subsequent neonatal behaviour and its influence on breastfeeding, only two out of 57 trials of opiates reported breastfeeding as an outcome. 
We therefore strongly recommend that the outcome measures, agreed through wide consultation for this project, are used in future trials of methods of pain management. Future trialists may, of course, wish to supplement these core outcome measures with additional topic-specific or trial-specific outcomes.

Further trials are needed particularly for non-pharmacological methods of pain management. There were very few data for hypnosis, biofeedback, sterile water injection, aromatherapy and massage with much $<1000$ women recruited in total to all trials of each method. For TENS, there were more trials and more women recruited, but also uncertainty about its value. In the UK at least, TENS is popular with women, very widely recommended by midwives, but unsupported by the national guideline developer, NICE. This discordance between the views of women, clinicians and guidelines reflects the poor evidence base and the uncertainty should be resolved by a definitive trial.

Pain management in labour is a very high priority for consumer groups. Health funding agencies need to consider if their priorities match with those of consumer groups.

\section{ACKNOWLEDGEMENTS}

David Tovey for his contributions to the development of the overview.
We thank the Editorial Staff of the Cochrane Pregnancy and Childbirth group for their help in preparing the overview.

We thank Gill Gyte, PCG Consumer Group Co-ordinator, for her help in writing the plain language summary for the overview.

NISCHR Cymru Children and Young People's Research Network supported Sue Jordan's travel to attend a meeting of the authors and members of the Cochrane Pregnancy and Childbirth Group in Liverpool in 2010.

As part of the pre-publication editorial process, this overview has been commented on by five peers (an editor and four referees who are external to the editorial team), five members of the Pregnancy and Childbirth Group's international panel of consumers and a statistical adviser.

The National Institute for Health Research (NIHR) is the largest single funder of the Cochrane Pregnancy and Childbirth Group. The views and opinions expressed therein are those of the authors and do not necessarily reflect those of the NIHR, NHS or the Department of Health. 


\section{RE F E R E N C E S}

\section{References to included reviews}

Anim-Somuah 2011

Anim-Somuah M, Smyth RMD, Jones L. Epidural versus non-epidural or no analgesia in labour. Cochrane Database of Systematic Reviews 2011, Issue 12. [DOI: 10.1002/14651858.CD000331.pub3]

\section{Barragán 2011}

Barragán Loayza IM, Solà I, Juandó Prats C. Biofeedback for pain management during labour. Cochrane Database of Systematic Reviews 2011, Issue 6. [DOI: 10.1002/14651858.CD006168.pub2]

\section{Cluett 2009}

Cluett ER, Burns E. Immersion in water in labour and birth. Cochrane Database of Systematic Reviews 2009, Issue 2. [DOI: 10.1002/14651858.CD000111.pub3]

\section{Derry 2012}

Derry S, Straube S, Moore RA, Hancock H, Collins SL. Intracutaneous or subcutaneous sterile water injection compared with blinded controls for pain management in labour. Cochrane Database of Systematic Reviews 2012, Issue 1. [DOI: 10.1002/14651858.CD009107.pub2]

\section{Dowswell 2009}

Dowswell T, Bedwell C, Lavender T, Neilson JP. Transcutaneous electrical nerve stimulation (TENS) for pain relief in labour. Cochrane Database of Systematic Reviews 2009, Issue 2. [DOI: 10.1002/14651858.CD007214.pub2]

\section{Halpern 2003a}

Halpern SH, Walsh V. Epidural ropivacaine versus bupivacaine for labor: a meta-analysis. Anesthesia and Analgesia 2003;96(5):1473.

\section{Hutton 2009}

Hutton EK, Kasperink M, Rutten M, Reitsma A, Wainman B. Sterile water injection for labour pain: a systematic review and meta-analysis of randomised controlled trials. BJOG: an international journal of obstetrics and gynaecology 2009;116(9):1158-66.

\section{Klomp 2012}

Klomp T, van Poppel M, Lazet J, Di Nisio M. Inhaled analgesia for pain management in labour. Cochrane Database of Systematic Reviews in press.

\section{Madden 2012}

Madden K, Middleton P, Cyna AM, Matthewson M. Hypnosis for pain management during labour and childbirth. Cochrane Database of Systematic Reviews in press.

\section{Mardirosoff 2002}

Mardirosoff C, Dumont L, Boulvain M, Tramer MR. Fetal bradycardia due to intrathecal opioids for labour analgesia: a systematic review. BJOG: an international journal of obstetrics and gynaecology 2002;109(3):274-81.

\section{Novikova 2012}

Novikova N, Cluver C. Local anaesthetic nerve block for pain management in labour. Cochrane Database of Systematic Reviews in press.

Othman 2012

Othman M, Jones L, Neilson JP. Non-opioid drugs for pain management in labour. Cochrane Database of Systematic Reviews in press.

\section{Simmons 2012}

Simmons SW, Cyna AM, Dennis AT, Hughes D. Combined spinal-epidural versus epidural analgesia in labour. Cochrane Database of Systematic Reviews in press.

\section{Smith 2011a}

Smith CA, Collins CT, Crowther CA, Levett KM. Acupuncture or acupressure for pain management in labour. Cochrane Database of Systematic Reviews 2011, Issue 7. [DOI: 10.1002/14651858.CD009232]

\section{Smith 2011b}

Smith CA, Levett KM, Collins CT, Crowther CA. Relaxation techniques for pain management in labour. Cochrane Database of Systematic Reviews 2011, Issue 12. [DOI: 10.1002/14651858.CD009514]

\section{Smith 2011c}

Smith CA, Collins CT, Crowther CA. Aromatherapy for pain management in labour. Cochrane Database of Systematic Reviews 2011, Issue 7. [DOI: 10.1002/14651858.CD009215]

\section{Smith 2012}

Smith CA, Levett KM, Collins CT, Jones L. Massage, reflexology and other manual methods for pain management in labour. Cochrane Database of Systematic Reviews 2012, Issue 2. [DOI: 10.1002/14651858.CD009290]

\section{Ullman 2010}

Ullman R, Smith LA, Burns E, Mori R, Dowswell T. Parenteral opioids for maternal pain management in labour. Cochrane Database of Systematic Reviews 2010, Issue 9. [DOI: 10.1002/14651858.CD007396.pub2]

\section{References to excluded reviews}

\section{Arnal 2009}

Arnal D, Serrano ML, Corral EM, Garcia del Valle S. Intravenous remifentanyl for labor analgesia [Remifentanilo intravenoso para analgesia del trabajo del parto.]. Revista Espanola de Anestesiologia y Reanimacion 2009;56(4):222-31.

\section{Aveline 2001}

Aveline C, Bonnet F. The effects of peridural anesthesia on duration of labor and mode of delivery [Influence de l'anesthesie peridurale sur la duree et les modalites de l'accouchement.]. Annales Francaises d'Anesthesie et de Reanimation 2001;20(5):471-84. 


\section{Benfield 2002}

Benfield RD. Hydrotherapy in labor. Journal of Nursing Scholarship 2002;34(4):347-52.

\section{Bricker 2002}

Bricker L, Lavender T. Parenteral opioids for labor pain relief: a systematic review. American Journal of Obstetrics and Gynecology 2002;186(5 Suppl Nature):S94-109.

\section{Bucklin 2002}

Bucklin BA, Chestnut DH, Hawkins JL. Intrathecal opioids versus epidural local anesthetics for labor analgesia: a meta-analysis. Regional Anesthesia and Pain Medicine 2002;27(1):23-30.

\section{Capogna 2004}

Capogna G, Camorcia M. Epidural analgesia for childbirth: effects of newer techniques on neonatal outcome. Paediatric Drugs 2004;6(6):375-86.

\section{Carroll 1997}

Carroll D, Moore RA, Tramer MR, McQuay HJ. Transcutaneous electrical nerve stimulation does not relieve labor pain: updated systematic review. Contemporary Reviews in Obstetrics and Gynaecology 1997;9(3):195-205.

\section{Carroll 1997a}

Carroll D, Tramer M, McQuay H, Nye B, Moore A. Transcutaneous electrical nerve stimulation in labour pain: a systematic review. British Journal of Obstetrics and Gynaecology 1997;104(2):169-75.

\section{Cho 2010}

Cho S-H, Lee H, Ernst E. Acupuncture for pain relief in labour: a systematic review and meta-analysis. BJOG: an international journal of obstetrics and gynaecology 2010;117(8):907-20.

\section{Choi 2003}

Choi PT, Galinski SE, Takeuchi L, Lucas S, Tamayo C, Jadad AR. PDPH is a common complication of neuraxial blockade in parturients: a meta-analysis of obstetrical studies. Canadian Journal of Anaesthesia 2003;50(5):460-9.

\section{Cyna 2004a}

Cyna AM, McAuliffe GL, Andrew MI. Hypnosis for pain relief in labour and childbirth: a systematic review. British Journal of Anaesthesia 2004;93(4):505-11.

\section{Fogarty 2008}

Fogarty V. Intradermal sterile water injections for the relief of low back pain in labour. A systematic review of the literature. Women and Birth 2008;21(4):157-63.

\section{Habib 2006}

Habib AS, Gan TJ. Use of neostigmine in the management of acute postoperative pain and labour pain: a review. CNS Drugs 2006;20(10):821-39.

\section{Hager 1999}

Hager A, Newton W. Comparing epidural and parenteral opioid analgesia during labor. Journal of Family Practice 1999;48(3):174-5.

\section{Halpern 1998}

Halpern SH, Leighton BL, Ohlsson A, Barrett JFR, Rice A. Effect of epidural vs parenteral opioid analgesia on the progress of labor. A meta-analysis. JAMA 1998;280(24):2105-10.

\section{Halpern 2003b}

Halpern SH, Leighton BL. Misconceptions about neuraxial analgesia. Anesthesiology Clinics of North America 2003;21(1):59-70.

\section{Halpern 2005a}

Halpern S. Low concentration epidural infusion increases the risk of instrumental vaginal delivery, but not cesarean delivery - meta-analysis. Evidence-Based Obstetrics and Gynecology 2005;7(2):66-7.

\section{Halpern 2005b}

Halpern S. Recent advances in patient-controlled epidural analgesia for labour. Current Opinion in Anaesthesiology 2005;18(3):247-51.

\section{Halpern 2009}

Halpern SH, Carvalho B. Patient-controlled epidural analgesia for labor. Anesthesia and Analgesia 2009;108(3):921-8.

\section{Hodnett 2002}

Hodnett ED. Pain and women's satisfaction with the experience of childbirth: a systematic review. American Journal of Obstetrics and Gynecology 2002;186(5 Suppl Nature):S160-72.

\section{Huang 2002}

Huang C, Macario A. Economic considerations related to providing adequate pain relief for women in labour: comparison of epidural and intravenous analgesia. PharmacoEconomics 2002;20(5):305-18.

\section{Huntley 2004}

Huntley AL, Coon JT, Ernst E. Complementary and alternative medicine for labor pain: a systematic review. American Journal of Obstetrics and Gynecology 2004;191(1):36-44.

\section{Koehn 2002}

Koehn ML. Childbirth education outcomes: an integrative review of the literature. Journal of Perinatal Education 2002;11(3):10-9.

\section{Kotaska 2006}

Kotaska AJ, Klein MC, Liston RM. Epidural analgesia associated with low-dose oxytocin augmentation increases cesarean births: a critical look at the external validity of randomized trials. American Journal of Obstetrics and Gynecology 2006;194(3):809-14.

\section{Kuczkowski 2004}

Kuczkowski KM. Neurologic complication of labor analgesia: facts and fiction. Obstetrical \& Gynecological Survey 2004;59(1):47-51. 


\section{Lally 2008}

Lally JE, Murtagh MJ, Macphail S, Thomson R. More in hope than expectation: a systematic review of women's expectations and experience of pain relief in labour. BMC Medicine 2008;6:7.

\section{Larkin 2009}

Larkin P, Begley CM, Devane D. Women's experiences of labour and birth: an evolutionary concept analysis. Midwifery 2009;25(2):e49-e59.

\section{Lee 2004}

Lee H, Ernst E. Acupuncture for labor pain management: a systematic review. American Journal of Obstetrics and Gynecology 2004;191(5):1573-9.

\section{Lee 2011}

Lee H, Cho S-H, Ernst E. 'Meta-analysis of randomised controlled trials (RCTs) involving acupuncture for labour pain shows acupuncture to be more effective than comparison treatments in analgesic consumption' but the overall evidence is still limited, calling for further investigations: Authors' reply. BJOG: an international journal of obstetrics and gynaecology 2011;118(1):101-2.

\section{Leeman 2003a}

Leeman L, Fontaine P, King V, Klein MC, Ratcliffe S. The nature and management of labor pain: Part I. Nonpharmacologic pain relief. American Family Physician 2003;68(6):1109-12.

\section{Leeman 2003b}

Leeman L, Fontaine P, King V, Klein MC, Ratcliffe S. The nature and management of labor pain: Part II. Pharmacologic pain relief. American Family Physician 2003;68(6):1115-22.

\section{Leighton 2002}

Leighton BL, Halpern SH. The effects of epidural analgesia on labor, maternal, and neonatal outcomes: a systematic review. American Journal of Obstetrics and Gynecology 2002;186(5 Suppl Nature):S69-77.

\section{Leighton 2003}

Leighton BL. The impact of neuraxial analgesia on the progress and outcome of labor. Techniques in Regional Anesthesia and Pain Management 2003;7(4):197-203.

\section{Lieberman 2002}

Lieberman E, O'Donoghue C. Unintended effects of epidural analgesia during labor: a systematic review. American Journal of Obstetrics and Gynecology 2002;186(5 Suppl Nature):S31-S68.

\section{Littleford 2004}

Littleford J. Effects on the fetus and newborn of maternal analgesia and anesthesia: a review. Canadian Journal of Anaesthesia 2004;51(6):586-609.

\section{Liu 2004}

Liu EHC, Sia ATH. Rates of caesarean section and instrumental vaginal delivery in nulliparous women after low concentration epidural infusions or opioid analgesia: systematic review. BMJ 2004;328(7453):1410-2.

\section{Lopard 2006}

Lopard E. Patient-controlled epidural analgesia for labour: in practice [L'analgesie peridurale obstetricale controlee par la patiente: en pratique]. Annales Francaises d'Anesthesie et de Reanimation 2006;25(6):593-8.

\section{Marucci 2007}

Marucci M, Cinnella G, Perchiazzi G, Brienza N, Fiore T. Patientrequested neuraxial analgesia for labor: impact on rates of cesarean and instrumental vaginal delivery. Anesthesiology 2007;106(5):1035-45.

\section{Mayberry 2002}

Mayberry LJ, Clemmens D, De A. Epidural analgesia adverse effects, co-interventions, and care of women during childbirth: a systematic review. American Journal of Obstetrics and Gynecology 2002;186(5 Suppl Nature):S81-S93.

\section{Minty 2007}

Minty RG, Kelly L, Minty A, Hammett DC. Single-dose intrathecal analgesia to control labour pain: is it a useful alternative to epidural analgesia?. Canadian Family Physician 2007;53(3):437-42.

\section{Morton 1994}

Morton SC, Williams MS, Keeler EB, Gambone JC, Kahn KL. Effect of epidural analgesia for labor on the cesarean delivery rate. Obstetrics \& Gynecology 1994;83(6):1045-52.

\section{Nystedt 2004}

Nystedt A, Edvardsson D, Willman A. Epidural analgesia for pain relief in labour and childbirth - a review with a systematic approach. Journal of Clinical Nursing 2004;13(4):455-66.

\section{Reynolds 2002}

Reynolds F, Sharma SK, Seed PT. Analgesia in labour and fetal acid-base balance: a meta-analysis comparing epidural with systemic opioid analgesia. BJOG: an international journal of obstetrics and gynaecology 2002;109(12):1344-53.

\section{Reynolds 2010}

Reynolds F. The effects of maternal labour analgesia on the fetus. Best Practice \& Research. Clinical Obstetrics \& Gynaecology 2010;24(3):289-302.

\section{Rosen 2002a}

Rosen Mark A. Nitrous oxide for relief of labor pain: a systematic review. American Journal of Obstetrics and Gynecology 2002;186(5 Suppl Nature):S110-S126.

\section{Rosen 2002b}

Rosen MA. Paracervical block for labor analgesia: a brief historic review. American Journal of Obstetrics and Gynecology 2002;186(5 Suppl Nature):S127-S130.

\section{Sharma 2003}

Sharma SK, Leveno KJ. Regional analgesia and progress of labor. Clinical Obstetrics and Gynecology 2003;46(3):633-45. 


\section{Sharma 2004}

Sharma SK, McIntire DD, Wiley J, Leveno KJ. Labor analgesia and cesarean delivery: an individual patient meta-analysis of nulliparous women. Anesthesiology 2004;100(1):142-8.

\section{Shiflett 2011}

Shiflett SC, Schwartz GE. Meta-analysis of randomised controlled trials (RCTs) involving acupuncture for labour pain shows acupuncture to be more effective than comparison treatments in several significant circumstances. BJOG: an international journal of obstetrics and gynaecology 2011;118(1):100-1.

\section{Simkin 2002}

Simkin PP, O'Hara MA. Nonpharmacologic relief of pain during labor: systematic reviews of five methods. American Journal of Obstetrics and Gynecology 2002;186(5 Suppl Nature):S131-S159.

\section{Simkin 2004a}

Simkin P, Bolding A. Update on nonpharmacologic approaches to relieve labor pain and prevent suffering. Journal of Midwifery \& Women's Health 2004;49(6):489-504.

\section{Sleth 2006}

Sleth J-C. Paracervical block in obstetrics [Bloc paracervical en obstetrique]. Annales Francaises d'Anesthesie et de Reanimation 2006;25(11-12):1119-26.

\section{Smith 2009}

Smith CA, Cochrane S. Does acupuncture have a place as an adjunct treatment during pregnancy? A review of randomized controlled trials and systematic reviews. Birth 2009;36(3):246-53.

\section{Thavaneswaran 2006}

Thavaneswaran P, Maddern G, Cooter R, Moyes D, Rudkin G. Paravertebral blocks for anaesthesia and analgesia: a systematic review. ASERNIP-S Report No. 47. Adelaide, South Australia: ASERNIP-S, January 2006. Royal Australasian College of Surgeons, Australian Safety and Efficacy Register of New Interventional Procedures (ASERNIP) - Surgical (http://www.surgeons.org/media/13814/ Paravertebral_Block_Review_March2006_web.pdf) (accessed 3 February 2011) 2006:263.

\section{Thorp 1996}

Thorp JA, Breedlove G. Epidural analgesia in labor: an evaluation of risks and benefits. Birth 1996;23(2):63-83.

\section{Van De Velde 2005}

Van De Velde M. Neuraxial analgesia and fetal bradycardia. Current Opinion in Anaesthesiology 2005;18(3):253-6.

\section{Van de Velde 2009}

Van de Velde M. Modern neuraxial labor analgesia: options for initiation, maintenance and drug selection. Revista Espanola de Anestesiologia y Reanimacion 2009;56(9):546-61.

\section{Van der Vyver 2002}

Van der Vyver M, Halpern S, Joseph G. Patient-controlled epidural analgesia versus continuous infusion for labour analgesia: a meta-analysis. British Journal of Anaesthesia 2002;89(3):459-65.

\section{Writer 1998}

Writer WDR, Stienstra R, Eddleston JM, Gatt SP, Griffin R, Gutsche BB, et al. Neonatal outcome and mode of delivery after epidural analgesia for labour with ropivacaine and bupivacaine: A prospective meta-analysis. British Journal of Anaesthesia 1998;81(5):713-7.

\section{Wunsch 2003}

Wunsch MJ, Stanard V, Schnoll SH. Treatment of pain in pregnancy. Clinical Journal of Pain 2003;19(3):148-55.

\section{Zhang 1999}

Zhang J, Klebanoff M A, DerSimonian R. Epidural analgesia in association with duration of labor and mode of delivery: a quantitative review. American Journal of Obstetrics and Gynecology 1999;180(4):970-7.

\section{Zhou 2008}

Zhou J, Lin X-M, Luo L-L, Ma Y-S. Local versus systemic application of opioids for labor analgesia: a systematic review. Chinese Journal of Evidence-Based Medicine 2008;8(8):652-61.

\section{Additional references}

\section{Ader 1990}

Ader L, Hansson B, Wallin G. Parturition pain treated by intracutaneous injections of sterile water. Pain 1990;41:133-8.

\section{Ahlborg 1996}

Ahlborg G Jr, Axelsson G, Bodin L. Shift work, nitrous oxide exposure and subfertility among Swedish midwives. International Journal of Epidemiology 1996;25(4):783-90.

\section{Aird 1997}

Aird IA, Luckas MJM, Buckett WM, Bousfield P. Effects intrapartum hydrotherapy on labour parameters. Australian and New Zealand Journal of Obstetrics and Gynaecology 1997;37(2):137-42.

\section{Allaire $\mathbf{2 0 0 0}$}

Allaire AD, Moos M, Wells SR. Complementary and alternative medicine in pregnancy: a survey of North Carolina nursemidwives. Obstetrics \& Gynecology 2000;95(1):19-23.

\section{AMA 1993}

American Medical Association. Reader's Guide to Alternative Health Methods. Milwaukee, WI: American Medical Association, 1993.

\section{Anim-Somuah 2005}

Anim-Somuah M, Smyth R, Howell C. Epidural versus non-epidural or no analgesia in labour. Cochrane Database of Systematic Reviews 2005, Issue 4. [DOI: 10.1002/14651858.CD000331.pub2]

\section{August 1961}

August RV. Hypnosis in Obstetrics. New York: McGraw-Hill, 1961. 


\section{Axelsson 1996}

Axelsson G, Ahlborg G Jr, Bodin L. Shift work, nitrous oxide exposure, and spontaneous abortion among Swedish midwives. Occupational and Environmental Medicine 1996;53(6):374-8.

\section{Bayarski 2006}

Bayarski Y. Differences between opioid and non-opioid analgesics. Ezine Articles (http://ezinearticles.com/?DifferencesBetween-Opioid-and-Non-Opioid-Anaglesics\&id=266768) (accessed November 2010) 2006.

\section{Begley 2009}

Begley C, Devane D, Clarke M. An evaluation of midwifery-led care in the Health Service Executive North Eastern Area. The report of the MidU study. Dublin: Trinity College, University of Dublin, 2009.

\section{Black 2009}

Marcovitch H (editor). Black's Medical Dictionary 42nd edition. http://www.library.nhs.uk/default.aspx (accessed 21st April 2011).

\section{BOC 2010}

BOC Healthcare. BOC Medical Gas Data Sheet (MGDS) entonox $50 \%$ nitrous oxide, $50 \%$ oxygen medicinal gas mixture SPC. MGDS (www.bocsds.com/uk/sds/medical/entonox.pdf) (accessed 30 January 2011) 2010.

\section{Bodin 1999}

Bodin L, Axelsson G, Ahlborg G Jr. The association of shift work and nitrous oxide exposure in pregnancy with birth weight and gestational age. Epidemiology 1999;10(4):429-36.

\section{Boivin 1997}

Boivin JF. Risk of spontaneous abortion in women occupationally exposed to anaesthetic gases: a meta-analysis. Occupational and Environmental Medicine 1997;54(8):541-8.

\section{Bonica 1990}

Bonica JJ. Definitions and taxonomy of pain. In: Bonica JJ editor(s). The Management of Pain. 2nd Edition. Philadelphia: Lea \& Febiger, 1990.

\section{Britton 2007}

Britton C, McCormick FM, Renfrew MJ, Wade A, King SE. Support for breastfeeding mothers. Cochrane Database of Systematic Reviews 2007, Issue 1. [DOI: 10.1002/14651858.CD001141.pub3]

\section{Brucker 1984}

Brucker MC. Nonpharmaceutical methods for relieving pain and discomfort during pregnancy. MCN, American Journal of Maternal Child Nursing 1984;9(6):390-4.

\section{Caton 2002}

Caton D, Corry MP, Frigoletto FD, Hopkins DP, Lieberman E, Mayberry $L$, et al. The nature and management of labor pain. American Journal of Obstetrics and Gynecology 2002;186(5 Suppl Nature):S1-S15.

\section{Clyburn 1993}

Clyburn P, Rosen M. The effect of opioid and inhalational analgesia on the newborn. In: Reynolds F editor(s). Effects on the Baby of Maternal Analgesia and Anaesthesia. London: Saunders, 1993:169-90.

\section{COMET 2001}

Comparative Obstetric Mobile Epidural Trial (COMET) Study Group UK. Effect of low dose mobile versus traditional epidural techniques on mode of delivery: a randomised control trial. Lancet 2001;358(9275):19-23.

\section{Cyna 2004}

Cyna AM, McAuliffe GL, Andrew MI. Hypnosis for pain relief in labour and childbirth: a systematic review. British Journal of Anaesthesia 2004;93(4):505-11. [PUBMED: 15277295]

\section{Dewhurst 2007}

Edmonds DK, Dewhurst J. Chapter 9: Analgesia and anaesthesia. Dewhurst's Textbook of Obstetrics and Gynaecology. 7th Edition. London: Wiley-Blackwell, 2007:63-8.

\section{Dick Read 1954}

Dick Read G. Childbirth Without Fear: The Principles and Practice of Natural Childbirth. 3rd Edition. London: William Heinemann, 1954.

\section{Dick Read 2004}

Dick Read G. Childbirth Without Fear: The Principles and Practice of Natural Childbirth. London: Pinter \& Martin, 2004.

\section{Edlich 1987}

Edlich RF, Towler MA, Goitz RJ, Wilder RP, Buschbacher LP, Morgan RF, et al. Bioengineering principles of hydrotherapy. Journal of Burn Care and Rehabilitation 1987;8:580-4.

\section{Eng 2006}

Eng YH, Cyna AM. A comparison of midwives' knowledge of and attitudes to hypnosis in hospitals with and without a hypnotherapy service. Australian Journal of Clinical Hypnotherapy and Hypnosis 2006;34(1):17-26.

\section{Ernst 1997}

Ernst E, Koeder K. An overview of reflexology. European Journal of General Practice 1997;3:52-7.

\section{Evron 2007}

Evron S, Ezri T. Options for systemic labor analgesia. Current Opinion in Anaesthesiology 2007;20:181-5.

\section{Faymonville 2000}

Faymonville ME, Laureys S, Degueldre C, DelFiore G, Luxen A, Franck G, et al. Neural mechanisms of antinociceptive effects of hypnosis. Anesthesiology 2000;92(5):1257-67.

\section{Findley 1999}

Findley I, Chamberlain G. ABC of labour care: relief of pain. BMJ 1999;318:927-30. 


\section{Foureur 2008a}

Foureur M. Theorising birth territory. In: Fahy K, Foureur M, Hastie $C$ editor(s). Birth Territory and Midwifery Guardianship: Theory for Practice, Education and Research. Edinburgh: Books for Midwives, 2008.

\section{Foureur 2008b}

Foureur M. Creating birth space to enable undisturbed birth. In: Fahy K, Foureur M, Hastie C editor(s). Birth Territory and Midwifery Guardianship: Theory for Practice, Education and Research. Edinburgh: Books for Midwives, 2008.

\section{Gamsa 2003}

Gamsa A. Hypnotic analgesia. In: Melzack R, Wall PD editor(s). Handbook of Pain Management: A Clinical Companion to Wall and Melzack's Textbook of Pain. Sydney: Churchill Livingstone, 2003:521-31.

\section{Garland 2000}

Garland D, Jones. Waterbirths: supporting practice with clinical audit. MIDIRS Midwifery Digest 2000;10(3):333-6.

\section{Gaskin 2003}

Gaskin IM. The pain/pleasure riddle. Ina May's Guide to Childbirth. New York: Bantam Dell, 2003:150-66.

\section{Geissbuehler 2000}

Geissbuehler V, Eberhard J. Waterbirths a comparative study. A prospective study on more than 2,000 waterbirths. Fetal Diagnosis and Therapy 2000;15(5):291-300.

\section{Gentz 2001}

Gentz BA. Alternative therapies for the management of pain in labor and delivery. Clinical Obstetrics \& Gynecology 2001;44(4):704-32.

\section{Ginesi 1998a}

Ginesi L, Niescierowicz R. Neuroendocrinology and birth 1: stress. British Journal of Midwifery 1998;6(10):659-63.

\section{Ginesi 1998b}

Ginesi L, Niescierowicz R. Neuroendocrinology and birth 2: The role of oxytocin. British Journal of Midwifery 1998;6(12):791-6.

\section{Gupta 2006}

Gupta JK, Hofmeyr GJ, Smyth RMD. Position in the second stage of labour for women without epidural anaesthesia. Cochrane Database of Systematic Reviews 2004, Issue 1. [DOI: 10.1002/14651858.CD002006.pub2]

\section{Hall 1998}

Hall SM, Holloway IM. Staying in control: women's experiences of labour in water. Midwifery 1998;14(1):30-6.

\section{Hatem 2008}

Hatem M, Sandall J, Devane D, Soltani H, Gates S. Midwifeled versus other models of care for childbearing women. Cochrane Database of Systematic Reviews 2008, Issue 4. [DOI: 10.1002/14651858.CD004667.pub2]

\section{Hebbes 2000}

Hebbes C, Lambert D. Non-opioid analgesic drugs. Anaesthesia \& Intensive Care 200;9(2):79-83.

\section{Higgins 2011}

Higgins JPT, Green S, editors. Cochrane Handbook for Systematic Reviews of Interventions Version 5.1.0 [updated March 2011]. The Cochrane Collaboration, 2011. Available from www.cochrane-handbook.org.

\section{Hodnett 2007}

Hodnett ED, Gates S, Hofmeyr GJ, Sakala C. Continuous support for women during childbirth. Cochrane Database of Systematic Reviews 2007, Issue 3. [DOI: 10.1002/14651858.CD003766.pub2]

\section{Hogg 1977}

Hogg MI, Wiener PC, Rosen M, Mapleson WW. Urinary excretion and metabolism of pethidine and norpethidine in the newborn. British Journal of Anaesthesia 1977;49(9):891-9.

\section{Hughes 2003}

Hughes D, Simmons SW, Brown J, Cyna AM. Combined spinal-epidural versus epidural analgesia in labour. Cochrane Database of Systematic Reviews 2003, Issue 4. [DOI: 10.1002/14651858.CD003401.pub2]

\section{Jadad 1996}

Jadad AR, Moore RA, Carroll D, Jenkinson C, Reynolds DJ, Gavaghan DJ, et al. Assessing the quality of reports of randomized clinical trials: is blinding necessary?. Controlled clinical trials 1996;17(1):1-12. [PUBMED: 8721797]

\section{Jones 2011}

Jones L, Dou L, Dowswell T, Alfirevic Z, Neilson James P. Pain management for women in labour: generic protocol. Cochrane Database of Systematic Reviews 2011, Issue 6. [DOI: 10.1002/14651858.CD009167]

\section{Jordan 2005}

Jordan S, Emery S, Bradshaw C, Watkins A, Friswell W. The impact of intrapartum analgesia on infant feeding. BJOG: an international journal of obstetrics and gynaecology 2005;112:927-34.

\section{Jordan 2010}

Jordan S. Pharmacology for Midwives: The Evidence Base for Safe Practice. 2nd Edition. Basingstoke: Palgrave/Macmillan, 2010.

\section{Kane 2002}

Kane RL, Bershadsky B, Lin WC, Rockwood T, Wood K. Efforts to standardize the reporting of pain. Journal of Clinical Epidemiology 2002;55:105-10.

\section{Kibuka 2009}

Kibuka M, Thornton JG, Kingswood CJ. Position in the second stage of labour for women with epidural anaesthesia. Cochrane Database of Systematic Reviews 2009, Issue 4. [DOI: 10.1002/14651858.CD008070] 


\section{KNOV 2009}

KNOV werkgroep Entonox. The use of Relivopan in primary care obstetrics [Adviesrapport: Het gebruik van Relivopan in de eerstelijns verloskunde]. Adviesrapport 2009; Vol. 3.

\section{Landolt 2011}

Landolt AS, Milling LS. The efficacy of hypnosis as an intervention for labor and delivery pain: a comprehensive methodological review. Clinical psychology review 2011;31(6):1022-31. [PUBMED: 21762655]

\section{Lawrence 2009}

Lawrence A, Lewis L, Hofmeyr GJ, Dowswell T, Styles C. Maternal positions and mobility during first stage labour. Cochrane Database of Systematic Reviews 2009, Issue 2. [DOI: 10.1002/14651858.CD003934.pub2]

\section{Leap 2004}

Leap N, Anderson T. The role of pain in normal birth and the empowerment of women. In: Downe S editor(s). Normal Childbirth: Evidence and Debate. Edinburgh: Churchill Livingstone, 2004:25-39.

\section{Leap 2008}

Leap N, Anderson P. The role of pain in normal birth and the empowerment of women. In: Downe S editor(s). Normal childbirth: evidence and debate. second edition. London: Churchill Livingstone, 2008.

\section{Leap 2010}

Leap N, Dodwell M, Newburn M. Working with pain in labour: an overview of evidence. New Digest 2010;49:22-6.

\section{Lechner 1991}

Lechner W, Jarosch E, Solder E, Waitz-Penz A, Mitterschiffthaler $\mathrm{G}$. Beta-endorphins during childbirth under transcutaneous electric nerve stimulation [Verhalten von Beta-Endorphin wahrend der Geburt unter transkutaner elektrischer Nervenstimulation]. Zentralblatt fur Gynakologie 1991;113(8):439-42.

\section{Lowe 2002}

Lowe NK. The nature of labor pain. American Journal of Obstetrics and Gynecology 2002;186:S16-S24.

\section{Lytzen 1989}

Lytzen T, Cederberg L, Moller-Nielsen J. Relief of low back pain in labor by using intracutaneous nerve stimulation (INS) with sterile water papules. Acta Obstetricia et Gynecologica Scandinavica 1989;68:341-3.

\section{Mankowski 2009}

Mankowski J, Kingston J, Moran T, Nager C, Lukacz E. Paracervical compared with intracervical lidocaine for suction curettage: a randomized controlled trial. Obstetrics \& Gynecology 2009;113(5):1052-7.

\section{Maquet 1999}

Maquet P, Faymonville ME, Degueldre C, Delfiore G, Luxen A, Franck $\mathrm{G}$, et al. Functional neuroanatomy of hypnotic state. Biological Psychiatry 1999;45(3):327-33.

\section{Marc 2011}

Marc I, Toureche N, Ernst E, Hodnett ED, Blanchet C, Dodin S, et al. Mind-body interventions during pregnancy for preventing or treating women's anxiety. Cochrane Database of Systematic Reviews 2011, Issue 7. [DOI: 10.1002/14651858.CD007559.pub2]

\section{Maze 2000}

Maze M, Fujinaga M. Recent advances in understanding the actions and toxicity of nitrous oxide. Anaesthesia 2000; Vol. 55, issue 4:311-4.

\section{Melzack 1965}

Melzack R, Wall PD. Pain mechanisms: a new theory. Science 1965;150(3699):971-9.

\section{Melzack 1984}

Melzack R. The myth of painless childbirth. Pain 1984;19(4):321-337.

\section{Moneta 2001}

Moneta J, Okninska A, Wielgos M, Przybos A, Szymusik I, Marianowski L. Patient's preferences concerning the course of labor. Ginekologia Polska 2001;72(12):1010-8.

\section{Mozingo 1978}

Mozingo JN. Pain in labor: a conceptual model for intervention. Journal of Obstetric, Gynecologic, and Neonatal Nursing 1978;7(4):47-9.

\section{NICE 2007}

National Institute for Health and Clinical Excellence. Intrapartum care: care of healthy women and their babies during childbirth. Clinical Guideline 55. London: NICE, 2007.

\section{NICE 2008}

National Collaborating Centre for Women's and Children's Health. Induction of labour. Clinical Guideline 70. London: NICE, 2008.

\section{Nissen 1995}

Nissen E, Lilja G, Matthiesen AS, Ransjo-Arvidsson AB, UvnasMoberg K, Widstrom AM. Effects of maternal pethidine on infants' developing breast feeding behaviour. Acta Paediatrica 1995;84(2):140-5.

\section{NLM 1991a}

National Library of Medicine. Labour stage, first. National Library of Medicine MeSH Browser (http:// www.ncbi.nlm.nih.gov/mesh/68007747) (accessed 24 January 2011) 1991.

\section{Ohlsson 2001}

Ohlsson G, Buchhave P, Leandersson U, Nordstrom L, Rydhstrom $\mathrm{H}$, Sjolin I. Warm tub bathing during labor: maternal and neonatal effects. Acta Obstetricia et Gynecologica Scandinavica 2001;80:311-4.

\section{Otigbah 2000}

Otigbah CM, Dhanjal MK, Harmsworth G. A retrospective comparison of water births and conventional vaginal deliveries. 
European Journal of Obstetrics \& Gynecology and Reproductive Biology 2000;91(1):15-20.

\section{Pace 2004}

Pace MC, Aurilio C, Bulletti C, lannotti M, Passavanti MB, Palagiano A. Subarachnoid analgesia in advanced labor: a comparison of subarachnoid analgesia and pudendal block in advanced labor: analgesic quality and obstetric outcome. Annals of the New York Academy of Sciences 2004;1034:356-63.

\section{Pomeranz 1989}

Pomeranz B, Stux G. Scientific Bases of Acupuncture. Berlin: Springer-Verlag, 1989.

\section{Rajan 1994}

Rajan L. The impact of obstetric procedures and analgesia/ anaesthesia during labour and delivery on breast feeding. Midwifery 1994;10:87-103.

\section{Ransjo-Arvidson 2001}

Ransjo-Arvidson AB, Matthiesen AS, Lilja G, Nissen E, Widstrom AM, Uvnas-Moberg K. Maternal analgesia during labor disturbs newborn behavior: effects on breastfeeding, temperature, and crying. Birth 2001;28(1):5-12.

\section{RevMan 2011 [Computer program]}

The Nordic Cochrane Centre, The Cochrane Collaboration. Review Manager (RevMan). Version 5.1. Copenhagen: The Nordic Cochrane Centre, The Cochrane Collaboration, 2011.

\section{Reynolds 2011}

Reynolds F. Labour analgesia and the baby: good news is no news. International Journal of Obstetric Anesthesia 2011;20(1):38-50.

Richmond 2003

Richmond H. Women's experiences of waterbirth. Practising Midwife 2003;6(3):26-31.

\section{Righard 1990}

Righard L, Alade MO. Effect of delivery room routines on success of first breast-feed. Lancet 1990;336:1105-7.

\section{Rosen 2002}

Rosen MA. Nitrous oxide for relief of labor pain: a systematic review. American Journal of Obstetrics and Gynecology 2002;186(5 Suppl Nature):S110-S126. [PUBMED: 12011877]

\section{Rosenfeld 1996}

Rosenfeld I. Dr. Rosenfeld's Guide to Alternative Medicine. New York: Random House, 1996.

\section{Russell 2000}

Russell R. The effects of regional analgesia on the progress of labour and delivery. British Journal of Anaesthesia 2000;84(6):799-12.

\section{Schorn 1993}

Schorn MN, McAllister JL, Blanco JD. Water immersion and the effect on labor. Journal of Nurse-Midwifery 1993;38(6):336-42.

\section{Sekhavat 2009}

Sekhavat L, Behdad S. The effects of meperidine analgesia during labor on fetal heart rate. International Journal of Biomedical Science 2009;5(1):59-62.

\section{Shea 2007}

Shea BJ, Grimshaw JM, Wells GA, Boers M, Andersson N, Hamel C, et al. Development of AMSTAR: a measurement tool to assess the methodological quality of systematic reviews. BMC Medical Research Methodology 2007;7:10. [PUBMED: 17302989]

\section{Simkin 2004}

Simkin P, Bolding A. Update on nonpharmacologic approaches to relieve labor pain and prevent suffering. Journal of Midwifery \& Women's Health 2004;49(6):489-504.

\section{Skibsted 1992}

Skibsted L, Lange AP. The need for pain relief in uncomplicated deliveries in an alternative birth center compared to an obstetric delivery ward. Pain 1992;48:183-6.

\section{Solt 2002}

Solt I, Ganadry S, Weiner Z. The effect of meperidine and promethazine on fetal heart rate indices during the active phase of labor. Israel Medical Association Journal 2002;4(3):178-80.

\section{Stevensen 1995}

Stevensen CJ. The psychophysiological effects of aromatherapy massage following cardiac surgery. Complementary Therapies in Medicine 1995;2:27-35.

\section{Thornton 2001}

Thornton JG. Reducing likelihood of instrumental delivery with epidural anaesthesia. Lancet 2001;358(9275):2.

\section{Torvaldsen 2006}

Torvaldsen S, Roberts CL, Simpson JM, Thompson JF, Ellwood DA. Intrapartum epidural analgesia and breastfeeding: a prospective cohort study. International Breastfeeding Journal 2006;1:24

\section{Trolle 1991}

Trolle B, Moller M, Kronborg H, Thomsen S. The effect of sterile water blocks on low back labor pain. American Journal of Obstetrics and Gynecology 1991;164:1277-81.

\section{Vickers 1999}

Vickers A, Zollman C. ABC of complementary therapies: massage therapies. BMJ 1999;319:1254-7.

\section{Wall 1967}

Wall PD, Sweet W. Temporary abolition of pain in man. Science 1967;155:108-9.

\section{Werner 1982}

Werner WE, Schauble PG, Knudson MS. An argument for the revival of hypnosis in obstetrics. American Journal of Clinical Hypnosis 1982;24(3):149-71. [PUBMED: 7137062] 


\section{Wiruchpongsanon 2006}

Wiruchpongsanon P. Relief of low back labor pain by using intracutaneous injections of sterile water: a randomized clinical trial. Journal of the Medical Association of Thailand 2006;89:571-6.

\section{ADDITIONAL TABLES}

\section{Table 1. Non-Cochrane reviews: characteristics of excluded reviews}

\begin{tabular}{lll}
\hline Name of Review & Description of review & $\begin{array}{l}\text { Reason for exclu- } \\
\text { sion }\end{array}$ \\
\hline Arnal 2009 & $\begin{array}{l}\text { This paper examined IV remifentanil for pain relief in labour. (The paper is in Spanish and } \\
\text { eligibility assessment was carried out using the abstract only). }\end{array}$ & $\begin{array}{l}\text { This review exam- } \\
\text { ines IV remifen- } \\
\text { tanil and this top- } \\
\text { ic is covered in a } \\
\text { recently updated } \\
\text { Cochrane review. }\end{array}$ \\
\hline Aveline 2001 & $\begin{array}{l}\text { This review examined epidural and combined spinal epidural. The main focus of the re- } \\
\text { view is on the duration of labour and the mode of delivery. It appeared that only five stud- } \\
\text { ies were included, although others were discussed in the text. There did not appear to } \\
\text { have been any systematic assessment of bias. (This paper was published in French, so } \\
\text { this assessment mainly relied on details in the abstract). }\end{array}$ & $\begin{array}{l}\text { The top of this } \\
\text { review is covered } \\
\text { in a recently up- } \\
\text { dated Cochrane } \\
\text { review. }\end{array}$
\end{tabular}

Benfield 2002 This review focused on hydrotherapy for pain relief in labour.There was no description of the methods used to carry out this review. There was no systematic search strategy or method of data extraction, no clear inclusion and exclusion criteria. There was no systematic assessment of risk of bias. All types of studies were included. There was no meta analysis, rather a narrative description of findings.

Bricker 2002 This systematic review examined parenteral opioids. Cochrane methods were used and analysis was carried out using Review Manager software.
This was not a systematic review.
The review appeared to have been carried out in a systematic manner. The search criteria, comparisons, inclusion and exclusion criteria were stated along with outcomes. It was not clear that there was a systematic quality assessment of studies. One measure of quality was stated to be publication in a peer reviewed journal. There was no mention of domain based quality assessment but in the tables in the review there were notes re study design that included risk of bias e.g.. It was stated if the study was blinded and whether staff collecting outcome data were blinded.

\section{Bucklin 2002}

This review was a mainly narrative summary of findings from RCTs and other reviews (with meta-analysis) focusing on various types of epidural and CSE. It examined different doses and types of drugs and comparisons. The main focus was on neonatal outcome. The methods of the review were not described. There was no search strategy specified, no clear inclusion and exclusion criteria, and no systematic assessment of quality.

This review examines TENS in labour. The review methods were described. The search
strategy and inclusion and exclusion criteria were specified. Outcomes included analgesic
effect and adverse effects. There was systematic assessment of study quality including
blinding and attrition. The review included 8 RCTs. The review was carried published in

Pain management for women in labour: an overview of systematic reviews (Review) Copyright $\odot 2013$ The Cochrane Collaboration. Published by John Wiley \& Sons, Ltd.
Not a systematic review.

A more recent Cochrane review examines TENS for pain relief in labour.
This systematic review of parenteral opioids, has been superseded by a recently updated Cochrane systematic review.

The topic of this review is covered in an included systematic review. 
Table 1. Non-Cochrane reviews: characteristics of excluded reviews (Continued)

1997 and the more recent Cochrane TENS review examined all of the eight included trials along with other more recent RCTs.

Carroll 1997a This is an update of Carroll 1997 and includes 10 RCTs.

A more recent Cochrane review examines TENS for pain relief in labour.

This review focusing on acupuncture for pain relief in labour uses Cochrane methods and
Review Manager software was used to carry out meta-analysis.

This review covers the same topic as a Cochrane review.

\begin{tabular}{ll}
\hline Choi & This review was not examining pain management in labour, rather it focused on the inci- \\
2003 & dence of headache following epidural.
\end{tabular}

The topic of this review is covered in a recently updated Cochrane review.

Cyna 2004a There has been a more recent Cochrane review on the same subject by the same author.

The topic of this review is covered in a recently updated Cochrane review.

Fogarty $2008 \quad \begin{aligned} & \text { This review on intradermal sterile water injections for the relief of low back pain in labour } \\ & \text { provides a narrative summary of findings from reviews. There is no meta-analysis and } \\ & \text { there has been a more recent Cochrane review on the same topic. }\end{aligned}$

The topic of this review is covered in a recently updated Cochrane review. of this review was not on women in labour.

Hager $1999 \quad$ This is a critique of an earlier review.

This is not a systematic review.

Halpern 1998 This is a systematic review of the effects of epidural versus parenteral opioid analgesia on
the progress of labour. There has been a more recent Cochrane review on the same sub-
ject, epidural versus non-epidural, which includes opioids as the non-epidural arm.

The topic of this review is covered in a recently updated Cochrane review. review is covered in a recently updated Cochrane review.

Halpern 2003b This is not a systematic review.

This is not a systematic review. ual RCTS. It was not clear that there was a systematic search or assessment of individual This is not a sysstudy quality. The review focuses on PCEA vs a continuous infusion. The review also distematic review. 
Table 1. Non-Cochrane reviews: characteristics of excluded reviews (Continued)

cusses studies where PCEA plus a continuous background infusion are compared with PCEA alone; and examines different types of PCEA drugs.

\section{Halpern 2009}

This review used systematic methods to assess different types of patient controlled epidural analgesia. The search methods were described (search 1998 to 2008). The review examined a range of different comparisons:

1. PCEA with background infusion vs no background infusion

2. PCEA ropivacaine vs PCEA bupivacaine

3. High vs low PCEA background doses/ lockout times and bolus doses, and different methods of administering drugs.

There was no meta- analysis. Main findings for each study are set out in tables and summarised in the text.
No data available from meta-analyses.
Hodnett 2002

This review focuses on all of the primary outcomes of the overview but examines broader questions and draws on descriptive studies. It provides a very useful summary of literature on satisfaction with pain relief.

Huang 2002
Not a systematic review including only RCTs.
This review examines economic issues relating to pain relief in labour a topic which is rarely addressed in trials.
This is not an effectiveness review.

This review covers areas already covered in included Cochrane reviews.

The review included clear inclusion, exclusion criteria, a search strategy and independent data extraction. There was an assessment of methodological quality (Jadad score). The review included RCTs and quasi RCTs. There was no meta-analysis due to "statistical heterogeneity amongst the included studies. Results are set out in tabular and narrative form.

This is a review looking at studies examining childbirth education/ antenatal classes. The reviewer used a systematic search mechanism although it was not clear how studies were selected for inclusion. The review included both qualitative and quantitative studies but $11 / 12$ studies were descriptive and there was one before and after study. No RCTs were included. There was no systematic evaluation of risk of bias.Some of the included studies included outcomes relating to pain perception and satisfaction with the childbirth experience, but this was not the main focus of the review. There was no meta analysis or quantitative summary of findings. Results were reported in narrative form and in descriptive tables.
This is not a systematic effectiveness review. Narrative summary of findings from mainly descriptive studies.
This review examines epidural versus parenteral opiates. The review included a search of MEDLINE and the The Cochrane Library. There was no systematic assessment of risk of bias. Results were reported in tables and in the text. A recently updated Cochrane review covers the same topic.
A recently updated Cochrane review covers the same topic.
Kuczkowski 2004 This is not a systematic review, it is a general summary of the literature on regional analgesia.
This is not a systematic review. 


\section{Table 1. Non-Cochrane reviews: characteristics of excluded reviews (Continued)}

Lally $2008 \quad$ This review used systematic methods but it is not an effectiveness review and included both qualitative and quantitative studies describing women's perceptions of pain relief during childbirth. Results were summarised in tables and the text.
This review included non-randomised studies.
Larkin 2009
This is not a systematic review but a qualitative analysis of papers reporting women's experiences of childbirth.
This is not a systematic review.

\begin{abstract}
Lee 2004
Systematic review examining acupuncture for pain relief in labour. There was a comprehensive search of 7 databases; assessed methodological quality. All included studies are in more recent Cochrane systematic review.
\end{abstract}

A recently updated Cochrane review covers the same topic.

This is not a systematic review.
Leeman 2003a

This paper focused on non-pharmacological methods; It was not clear that this was a systematic review, there was no description of review methods. It was not clear if there was a search strategy. There was some description of the quality of the included studies but this was not systematic. There was no meta-analysis. Findings were set out in tables and text. The review included Cochrane and other systematic reviews along with other RCTs. It focused on support in labour, sterile water injection, massage, baths and warm water. This review has been superceded by more recent Cochrane reviews.
This is not a systematic review, rather a narrative summary of findings from reviews and trials.

This is not a systematic review.
This is an update of a well-conducted systematic review (Halpern 1998). The review focuses on epidural vs parenteral opioids in labour in relation to progress in labour and rate of caesarean section. A systematic search was carried out (1980-2001) and details of the search strategy and results of the search are provided. There was independent data extraction by two reviewers and a systematic assessment of quality (Jadad score). The review includes meta-analysis. This review is now out of date and has been superseded by a more recent Cochrane review.
Superseded by more recent Cochrane review.
Leighton 2003
Not a systematic review but draws on findings of earlier systematic review by same author.
This review included a search strategy and systematic methods for extracting data. There was no systematic assessment of study quality using a domain-based risk of bias assessment tool, rather the strengths and weaknesses of study designs and analyses were considered. The review looked at both RCTs and observational studies comparing epidural vs opioids and epidural vs no epidural. Outcomes included rates of CS, assisted delivery,
More recent Cochrane reviews examine the same comparisons in RCTs. 
Table 1. Non-Cochrane reviews: characteristics of excluded reviews (Continued)

duration of labour and adverse neonatal outcomes. More recent Cochrane reviews have examined these comparisons.

Littleford $2004 \quad$ This is a thorough review of a range of methods for pain relief in labour. The main focus of the review is on adverse effects of analgesia on the fetus and newborn. Although there Not a systematic was a systematic literature search there was no systematic assessment of risk of bias. The review cited other reviews and included discussion of both randomised and non-randomised studies. There was no meta-analysis and results are presented in narrative form.

\section{Liu 2004}

\begin{abstract}
This systematic review examined low-dose epidural compared with opioids in nulliparous women. Outcomes included rates of CS, assisted delivery, duration of labour. Systematic methods; search strategy described, included RCTs only, systematic assessment of study quality using Scottish intercollegiate guideline network checklist (which includes the same broad areas of risk of bias as the Cochrane risk of bias tool); systematic methods for data extraction and meta-analysis. The focus is on nulliparous women only and the comparison has been examined in a more recent Cochrane review.
\end{abstract}

This is not a systematic review. It was not clear that there was a systematic search strategy or clear inclusion and exclusion criteria. There was no systematic assessment of risk of bias. The results are reported as a narrative summary. review.

More recent Cochrane review available.
Lopard 2006
This is not a systematic review.

\begin{abstract}
Marucci 2007
This review looks at epidural and combined spinal epidural in nulliparous women. The main outcomes are CS, assisted delivery and neonatal and newborn outcomes. Included both RCTs and cohort studies. The review used systematic methods; the search strategy was described (1990-2006). The studies were assessed for quality using standard measures (Jahad score for trials). There were standard methods used for data collection and meta-analysis was performed.
\end{abstract}

Mayberry 2002

This review looks at adverse effects of epidural. Included RCTs. The review used system-
Recently up-dated Cochrane reviews covers the same area. atic methods; the search strategy was described (1990-2000). The studies were assessed for quality. Standard methods were used for data collection. Meta-analysis was not performed rather the results are described narratively. Recently up-dated Cochrane reviews cover the same area.

Topic covered in recently updated Cochrane reviews.
This review focuses on intrathecal analgesia as an alternative to epidural. There was a systematic search and the strategy is described. The review includes all study types (reviews, RCTs and observational studies) and there was no clear systematic assessment of the quality of the evidence. The review covers a topic not covered by Cochrane reviews but without a clear assessment of risk of bias results are difficult to interpret.
Morton 1994 This review looks at epidural in nulliparous women. The main outcomes are CS, assisted delivery and neonatal and newborn outcomes. Included both RCTs and other types of studies. The review used some systematic methods; the search strategy was described There were standard methods used for data collection and meta-analysis was performed. There was no systematic assessment of study quality and risk of bias. Recently up-dated Cochrane reviews cover the same area.
The review includes a range of study designs.

Review includes non-randomised studies, no systematic assessment of study quality. Topic covered in more up to date Cochrane reviews.

\footnotetext{
Nystedt 2004

This review looks at the use of epidural.. Included both RCTs and other types of studies. The review used some systematic methods; the search strategy was described. The
}

Recently up-dated Cochrane reviews 
Table 1. Non-Cochrane reviews: characteristics of excluded reviews (Continued)

studies were assessed for quality based on study design (high, moderate or low scientific quality) but there was no systematic assessment of risk of bias. Recently up-dated Cochrane reviews cover the same area cover the same area.
Reynolds 2002
This review examines epidural vs parenteral opioids; the primary focus is on studies reporting fetal acid-base as an outcome. The review includes both randomised trials and non-randomised studies. A search strategy is described but there did not appear to be any systematic assessment of study quality. The topic of this review is covered in a recently updated Cochrane review.
The topic of this review is covered in a recently updated Cochrane review.
This review looks at paracervical block. A search strategy was described but the author describes the review as non-exhaustive. The search included MEDLINE and the Cochrane library. The studies included RCTs to examine the effects of analgesia on fetal bradycardia but other studies were included to describe adverse effects. There was no systematic assessment of study quality. The topic of this review has been covered in a recently updated Cochrane review.
The topic of this review has been covered in a recently updated Cochrane review.
This review looks inhaled analgesia. A search strategy was described. The search included MEDLINE and The Cochrane Library. The studies included RCTs but other studies were included to describe adverse effects. It was not clear that there was a systematic assessment of study quality but included studies were described as "low to moderate risk of bias". The topic of this review has been covered in a recently updated Cochrane review.
The topic of this review has been covered in a recently updated Cochrane review.
This review examines epidural analgesia drawing on evidence from randomised trials and non-randomised studies. It is not a systematic review and it was not clear that there was a search strategy or a systematic assessment of study quality.
This not a systematic review. Regional analgesia is covered in recently updated Cochrane Reviews.

This is not a systematic review. individual patient data collected in a series of trials over a 7 year period in the same hospital; a subset of nulliparous women are included in the sample in this meta-analysis. There was no search and no systematic assessment of risk of bias.

Shiflett $2011 \quad$ This is not a review. It is a letter commenting on the findings of another review. Cho acupuncture review.

This is not a review.

Simkin 2002

This review focuses on five methods to relieve pain in labour including broad based interventions such as continuous support in labour, warm baths, maternal positions and mobility in labour and massage and intradermal water. It included RCTs and non-randomised studies including before and after studies. There was a search strategy but there was no clear assessment of risk of bias. There was no meta-analysis. Results were presented in narrative form and in tables. The review includes broad base interventions which we have not included as methods of pain relief in labour; massage and water injections are covered in recently updated Cochrane reviews.
Includes non randomised studies and focuses on broad based interventions. 


\section{Table 1. Non-Cochrane reviews: characteristics of excluded reviews (Continued)}

Simkin 2004a This overview focuses on 13 non-pharmacological methods to relieve pain in labour including broad based interventions such as continuous support in labour, warm baths, maternal positions and mobility in labour and massage and intradermal water. It draws on findings from a series of systematic reviews (including Cochrane reviews). There was a search strategy but there was no clear assessment of risk of bias in the reviews included. There was no meta-analysis. Results were presented in narrative form and in tables. The review includes broad base interventions which we have not included as methods of pain relief in labour; massage and water injections and relaxation methods are covered in recently updated Cochrane reviews.

Sleth 2006 This is not a systematic review. There was a search strategy but the more recent data was selected to underpin this discussion of paracervical block. The topic is covered in a recently updated Cochrane review. (Assessment from English abstract; original full article published in French).
This is not a systematic review. It is a narrative review.
Not a systematic review. Cochrane review covers the same topic. the author of a recently updated Cochrane review on acupuncture in labour included in this overview.

Topic covered in recently updated Cochrane review.

Thavaneswaran 2006
This systematic review looks at the safety and efficacy of thoracic and lumbar paravertebral block in surgical patients. The review does not examine pain relief during labour.
This review examines pain relief for surgery (not during childbirth).
This review included evidence from both RCTs and non-randomised studies. There was a search strategy but no systematic assessment of risk of bias or study quality. The review focuses on epidural analgesia which is covered in recently updated Cochrane reviews.
The review focuses on epidural analgesia which is covered in recently updated Cochrane reviews.

This is not a systematic review.

This is not a systematic review.

Van de Velde 2009 This is not a systematic review but a general narrative summary and discussion of methods of neuraxial analgesia in labour.

This topic is covered by more recent reviews on epidural.

This is not a systematic review. sults pooled in a meta-analysis.
This is not a systematic review.

This topic is covered by more recent Cochrane reviews on epidural. 
Table 1. Non-Cochrane reviews: characteristics of excluded reviews (Continued)

Zhou 2008 This review includes RCTs comparing local versus systemic application of opioids for labour analgesia.
This topic is covered by a more recent Cochrane review.

CS: Caeserean section; PCEA: Patient Controlled Epidural Analgesia; RCT: randomised controlled trial

Table 2. Characteristics of included Cochrane systematic reviews - non-pharmacological interventions

\begin{tabular}{|c|c|c|c|c|c|c|}
\hline $\begin{array}{l}\text { Review } \\
\text { title }\end{array}$ & $\begin{array}{l}\text { Date of } \\
\text { search }\end{array}$ & $\begin{array}{l}\text { No. Stud- } \\
\text { ies includ- } \\
\text { ed } \\
\text { (No. Pa- } \\
\text { tients in } \\
\text { included } \\
\text { studies) }\end{array}$ & $\begin{array}{l}\text { Inclu- } \\
\text { sion cri- } \\
\text { teria for } \\
\text { "Types } \\
\text { of partici- } \\
\text { pants" }\end{array}$ & $\begin{array}{l}\text { Comparison inter- } \\
\text { ventions (no. stud- } \\
\text { ies) }\end{array}$ & $\begin{array}{l}\text { Outcomes for } \\
\text { which data } \\
\text { were reported } \\
\text { that could be } \\
\text { included in an } \\
\text { analysis }\end{array}$ & $\begin{array}{l}\text { Summary of quality of evidence } \\
\text { in reviews (risk of bias) }\end{array}$ \\
\hline $\begin{array}{l}\text { Hypnosis } \\
\text { for pain } \\
\text { manage- } \\
\text { ment dur- } \\
\text { ing labour } \\
\text { and child- } \\
\text { birth } \\
\text { (Madden } \\
\text { 2012) }\end{array}$ & $\begin{array}{l}7 \text { Septem- } \\
\text { ber } 2011\end{array}$ & $\begin{array}{l}7 \text { studies in } \\
3 \text { countries } \\
\text { (Australia, } \\
\text { USA, UK) } \\
\text { between } \\
1986 \text { and } \\
2010 \\
(1213 \\
\text { women) }\end{array}$ & $\begin{array}{l}\text { Pregnant } \\
\text { women } \\
\text { including } \\
\text { women in } \\
\text { high risk } \\
\text { groups } \\
\text { e.g. } \\
\text { preterm } \\
\text { labour or } \\
\text { following } \\
\text { induction } \\
\text { of labour }\end{array}$ & $\begin{array}{l}\text { Self-hypnosis or hyp- } \\
\text { notherapy versus } \\
\text { control (standard } \\
\text { childbirth prepara- } \\
\text { tion; a relaxation } \\
\text { tape; supportive } \\
\text { counselling; psy- } \\
\text { chotherapy) ( } 7 \text { stud- } \\
\text { ies, } 1070 \text { women) } \\
\text { Hypnosis \& audio CD } \\
\text { versus audio CD of } \\
\text { hypnosis ( } 1 \text { study, } \\
297 \text { women) } \\
\text { N.B. one study, } n= \\
448, \text { included three } \\
\text { arms and so data for } \\
\text { the different arms } \\
\text { are included in the } \\
\text { separate compar- } \\
\text { isons }\end{array}$ & $\begin{array}{l}\text { - Pain intensi- } \\
\text { ty } \\
\text { - Satisfaction } \\
\text { with pain re- } \\
\text { lief } \\
\text { - Satisfaction } \\
\text { with child- } \\
\text { birth experi- } \\
\text { ence } \\
\text { - Breastfeed- } \\
\text { ing } \\
\text { - Assisted } \\
\text { vaginal birth } \\
\text { - Caesarean } \\
\text { section } \\
\text { - Admission to } \\
\text { special care } \\
\text { baby unit/ } \\
\text { neonatal in- } \\
\text { tensive care } \\
\text { Apgar score } \\
\text { less than } \\
\text { seven at five } \\
\text { minutes } \\
\text { Adverse ef- } \\
\text { fects for } \\
\text { women and } \\
\text { infants } \\
\text { (postnatal } \\
\text { depres- } \\
\text { sion,new- } \\
\text { born resusci- } \\
\text { tation) }\end{array}$ & $\begin{array}{l}\text { Sequence generation: } 2 \text { studies } \\
\text { low risk; } 5 \text { studies unclear risk } \\
\text { Allocation concealment: } 1 \text { study } \\
\text { low risk; } 2 \text { studies high risk; } 4 \\
\text { studies unclear risk } \\
\text { Blinding (participants \& clinical } \\
\text { staff): } 3 \text { studies low risk; } 4 \text { stud- } \\
\text { ies unclear risk } \\
\text { Blinding (outcome assessors): } 3 \\
\text { studies low risk; } 4 \text { studies unclear } \\
\text { risk } \\
\text { Incomplete outcome reporting: } \\
3 \text { studies low risk; } 2 \text { studies high } \\
\text { risk; } 2 \text { studies unclear risk } \\
\text { Selective outcome reporting: } \\
3 \text { studies low risk; } 2 \text { studies high } \\
\text { risk; } 2 \text { studies unclear risk } \\
\text { Other potential threats to va- } \\
\text { lidity: } 3 \text { studies low risk; } 4 \text { stud- } \\
\text { ies unclear risk }\end{array}$ \\
\hline $\begin{array}{l}\text { Biofeed- } \\
\text { back for } \\
\text { pain dur- } \\
\text { ing labour }\end{array}$ & $\begin{array}{l}20 \text { March } \\
2011\end{array}$ & $\begin{array}{l}4 \text { studies in } \\
3 \text { countries } \\
\text { (UK, USA } \\
\text { and Italy) } \\
\text { between }\end{array}$ & $\begin{array}{l}\text { Women } \\
\text { with low } \\
\text { risk preg- } \\
\text { nan- } \\
\text { cies e.g. }\end{array}$ & $\begin{array}{l}\text { Biofeedback ver- } \\
\text { sus no biofeed- } \\
\text { back/standard } \\
\text { care ( } 2 \text { studies, } 103 \\
\text { women) }\end{array}$ & $\begin{array}{l}\text { - Assisted } \\
\text { vaginal birth } \\
\text { - Caesarean } \\
\text { section }\end{array}$ & $\begin{array}{l}\text { Sequence generation: } 1 \text { study } \\
\text { low risk; } 3 \text { studies unclear risk } \\
\text { Allocation concealment: } 4 \text { stud- } \\
\text { ies unclear risk }\end{array}$ \\
\hline
\end{tabular}


Table 2. Characteristics of included Cochrane systematic reviews - non-pharmacological interventions (Continued)

(Barragán

2011)
1978 and healthy

2000 pregnant

(201 women

women have a

ran- normal

domised, birth

2 stud-

ies $(103$

women)

contributed

data to

meta-

analysis)
Blinding: 4 studies unclear risk

Incomplete outcome reporting:

2 studies low risk; 2 studies high risk

Selective outcome reporting: 1 study low risk; 3 studies high risk

Other potential threats to va-

lidity: 4 studies low risk

\begin{tabular}{|c|c|c|c|c|c|}
\hline $\begin{array}{l}\text { Intracu- } \\
\text { taneous } \\
\text { or subcu- } \\
\text { taneous } \\
\text { sterile wa- } \\
\text { ter injec- } \\
\text { tion for } \\
\text { pain man- } \\
\text { agement } \\
\text { in labour } \\
\text { (Derry } \\
\text { 2012) }\end{array}$ & $\begin{array}{l}30 \text { May } \\
2011\end{array}$ & $\begin{array}{l}7 \text { studies in } \\
5 \text { countries } \\
\text { (Sweden, } \\
\text { Denmark, } \\
\text { Iran, Thai- } \\
\text { land, India) } \\
\text { between } \\
1990 \text { and } \\
2009 \\
\text { (766 } \\
\text { women) }\end{array}$ & $\begin{array}{l}\text { Women } \\
\text { in active } \\
\text { labour } \\
\text { who re- } \\
\text { quested } \\
\text { analgesia } \\
\text { for pain of } \\
\text { moderate } \\
\text { to severe } \\
\text { intensi- } \\
\text { ty. There } \\
\text { were no } \\
\text { restric- } \\
\text { tions re- } \\
\text { lating to } \\
\text { place of } \\
\text { birth or } \\
\text { to mater- } \\
\text { nal parity, } \\
\text { risk sta- } \\
\text { tus, age, } \\
\text { weight, } \\
\text { length of } \\
\text { gestation, } \\
\text { or stage of } \\
\text { labour }\end{array}$ & $\begin{array}{l}\text { Intracutaneous ster- } \\
\text { ile water injection } \\
\text { versus normal saline } \\
\text { injection ( } 4 \text { studies, } \\
467 \text { women) } \\
\text { Subcutaneous ster- } \\
\text { ile water injection } \\
\text { versus normal saline } \\
\text { injection ( } 2 \text { studies, } \\
200 \text { women) } \\
\text { Intracutaneous \& } \\
\text { subcutaneous sterile } \\
\text { water injection ver- } \\
\text { sus normal saline in- } \\
\text { jection (1 study, } 99 \\
\text { women) }\end{array}$ & $\begin{array}{ll}\text { - } & \text { Assisted } \\
& \text { vaginal birth } \\
\text { - } & \text { Caesarean } \\
& \text { section }\end{array}$ \\
\hline
\end{tabular}

Sequence generation: 4 studies

low risk; 3 studies unclear risk

Allocation concealment: 4 studies low risk; 4 studies unclear risk

Blinding (participants \& clinical staff): 5 studies low risk; 1 study high risk; 1 study unclear risk

Blinding (outcome assessors): 7 studies low risk

Incomplete outcome reporting: 2 studies low risk; 1 study high risk; 4 studies unclear risk

Selective outcome reporting: 4 studies low risk; 3 studies unclear risk

Other potential threats to validity: 3 studies high risk; 4 studies unclear risk

\begin{tabular}{|c|c|c|c|c|c|c|}
\hline $\begin{array}{l}\text { Immer- } \\
\text { sion in } \\
\text { water in } \\
\text { labour } \\
\text { and birth } \\
\text { (Cluett } \\
\text { 2009) }\end{array}$ & $\begin{array}{l}30 \text { June } \\
2011\end{array}$ & $\begin{array}{l}12 \text { stud- } \\
\text { ies in } 11 \\
\text { countries } \\
\text { (USA, Cana- } \\
\text { da, UK, } \\
\text { Sweden, } \\
\text { Finland, } \\
\text { Belguim, } \\
\text { Iran, South } \\
\text { Africa, } \\
\text { Brazil, Aus- } \\
\text { tralia) be- } \\
\text { tween } 1993 \\
\text { and } 2009 \\
\text { (3252 } \\
\text { women) }\end{array}$ & $\begin{array}{l}\text { Nulli- } \\
\text { parous } \\
\text { or mul- } \\
\text { tiparous } \\
\text { women } \\
\text { in labour } \\
\text { with a sin- } \\
\text { gleton } \\
\text { pregnan- } \\
\text { cy, irre- } \\
\text { spective } \\
\text { of gesta- } \\
\text { tion or } \\
\text { labour } \\
\text { character- } \\
\text { istics }\end{array}$ & $\begin{array}{l}\text { Immersion versus } \\
\text { no immersion in the } \\
\text { first stage of labour } \\
\text { ( } 8 \text { studies, } 2826 \\
\text { women) } \\
\text { Immersion versus } \\
\text { no immersion in } \\
\text { the second stage of } \\
\text { labour ( } 3 \text { studies, } 286 \\
\text { women) } \\
\text { Early versus late im- } \\
\text { mersion during the } \\
\text { first stage of labour } \\
\text { (1 study, } 200 \text { women) } \\
\text { N.B. one study, } n= \\
60 \text {, included data for }\end{array}$ & $\begin{array}{l}\text { - Pain intensi- } \\
\text { ty } \\
\text { - Satisfaction } \\
\text { with child- } \\
\text { birth experi- } \\
\text { ence } \\
\text { - Breastfeed- } \\
\text { ing } \\
\text { - Assisted } \\
\text { vaginal birth } \\
\text { - Caesarean } \\
\text { section } \\
\text { - Adverse ef- } \\
\text { fects for } \\
\text { women }\end{array}$ & $\begin{array}{l}\text { Sequence generation: } 6 \text { stud- } \\
\text { ies low risk; } 2 \text { studies high risk; } 4 \\
\text { studies unclear risk } \\
\text { Allocation concealment: } 8 \text { stud- } \\
\text { ies low risk; } 3 \text { studies high risk; } 1 \\
\text { study unclear risk } \\
\text { Blinding (participants/clini- } \\
\text { cal staff/outcome assessor): } 12 \\
\text { studies high risk } \\
\text { Incomplete outcome reporting: } \\
11 \text { studies low risk; } 1 \text { study un- } \\
\text { clear risk } \\
\text { Selective outcome reporting: } \\
10 \text { studies low risk; } 1 \text { study high } \\
\text { risk; } 1 \text { study unclear risk }\end{array}$ \\
\hline
\end{tabular}


Table 2. Characteristics of included Cochrane systematic reviews - non-pharmacological interventions (Continued)

both first and sec-

ond stages of labour

and so is included

in numbers for both

comparisons
- Adverse effects for infants

- Admission to special care baby unit/ neonatal intensive care unit

- Apgar score less than seven at five minutes

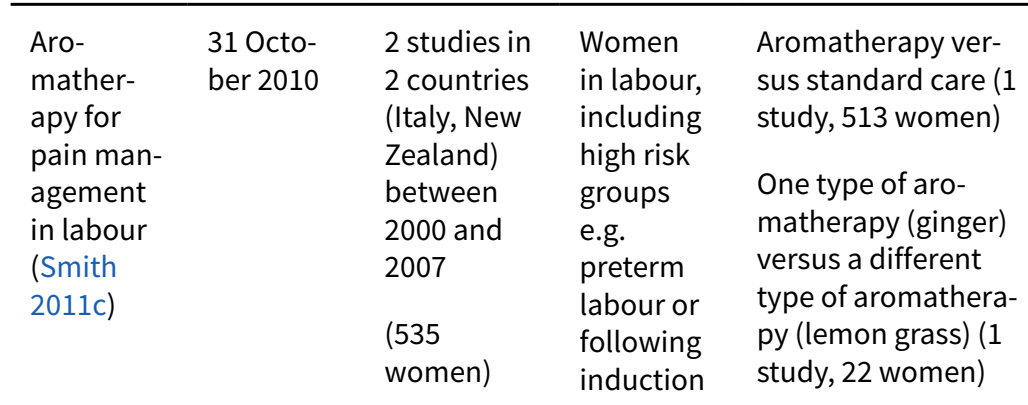

- Assisted vaginal birth

- Caesarean section

- Adverse effects for women (postpartum haemorrhage)

- Admission to special care baby unit

- Apgar score less than seven at five minutes
Other potential threats to va-

lidity: 1 study low risk; 11 studies unclear risk

Sequence generation: 2 studies low risk

Allocation concealment: 2 studies low risk

Blinding: 1 study low risk; 1 study high risk

Incomplete outcome reporting: 2 studies low risk

Selective outcome reporting: 2 studies unclear risk

Other potential threats to validity: 1 study low risk; 1 study high risk

- Pain intensi-

Sequence generation: 6 studies

Relax-

ation

30 November

11 studies in 9 countries (USA, UK, Sweden, Italy, Turkey, Thailand, Iran, Taiwan, Brazil) between 1965 and 2010

(1574 women) ty
Women Relaxation (progres-

in labour, including high risk groups

e.g. preterm labour or following induction sive muscle relaxation; breathing; psychoprophylaxis) versus standard care (6 studies,1147 women)

Yoga versus standard care (2 studies, 270 women)

Music versus standard care (2 studies, 133 women)

Audio-analgesia versus standard care (1 study, 24 women)
- Satisfaction with pain relief

- Satisfaction with childbirth experience

- Assisted vaginal delivery

- Caesarean section

- Apgar score less than seven at five minutes

- Pain Intensity

- Satisfaction with pain relief

- Satisfaction with child-

\section{low risk; 5 studies unclear risk}

Allocation concealment: 3 studies low risk; 8 studies unclear risk

Blinding: 8 studies high risk; 3 studies unclear risk

Incomplete outcome reporting: 5 studies low risk; 2 studies high risk; 4 studies unclear risk

Selective outcome reporting: 1 study low risk; 10 studies unclear risk

Other potential threats to validity: 5 studies low risk; 6 studies unclear risk

Sequence generation: 12 stud ies low risk; 1 study unclear risk

Allocation concealment: 9 studies low risk; 4 studies unclear risk den, Den- groups (12 studies, 1858 mark, In- e.g. women) dia, Korea, preterm agement

in labour 
Table 2. Characteristics of included Cochrane systematic reviews - non-pharmacological interventions (Continued)

(Smith

2011a)
Taiwan,

China, Iran)

between

2002 and

2010

(1986

women)

\section{labour or \\ Acupuncture ver-}

following

induction

sus sterile water in-

jection ( 1 study, 128

women) birth experi-

ence

- Assisted vaginal birth

- Caesarean section

- Apgar score less than seven at five minutes
Blinding: 3 studies low risk; 5 studies high risk; 5 studies unclear risk

Incomplete outcome reporting: 9 studies low risk; 1 study high risk; 3 studies unclear risk

Selective outcome reporting:

13 studies unclear risk

Other potential threats to validity: 8 studies low risk; 3 studies high risk; 2 studies unclear risk

\begin{tabular}{|c|c|c|c|c|}
\hline $\begin{array}{l}\text { Massage, } \\
\text { reflexol- } \\
\text { ogy and } \\
\text { other } \\
\text { manual } \\
\text { methods } \\
\text { for pain } \\
\text { manage- } \\
\text { ment in } \\
\text { labour } \\
\text { (Smith } \\
\text { 2012) }\end{array}$ & $\begin{array}{l}30 \text { June } \\
2011\end{array}$ & $\begin{array}{l}6 \text { studies } \\
\text { in } 4 \text { coun- } \\
\text { tries (USA, } \\
\text { UK, Iran, } \\
\text { Taiwan) be- } \\
\text { tween } 1997 \\
\text { and } 2010 \\
\text { (401 } \\
\text { women } \\
\text { ran- } \\
\text { domised, } \\
5 \text { stud- } \\
\text { ies ( } 326 \\
\text { women) } \\
\text { contributed } \\
\text { data to } \\
\text { meta- } \\
\text { analysis) }\end{array}$ & $\begin{array}{l}\text { Women } \\
\text { in labour, } \\
\text { including } \\
\text { high risk } \\
\text { groups } \\
\text { e.g. } \\
\text { preterm } \\
\text { labour or } \\
\text { following } \\
\text { induction }\end{array}$ & $\begin{array}{l}\text { Massage versus usual } \\
\text { care (4 studies, 225) } \\
\text { Massage versus } \\
\text { breathing exercises } \\
\text { (1 study, } 28 \text { women) } \\
\text { Massage versus mu- } \\
\text { sic (1 study, } 101 \\
\text { women) }\end{array}$ \\
\hline
\end{tabular}

- Pain intensity

- Satisfaction with pain relief

- Sense control labour

- Assisted vaginal birth

- Caesarean section

- Admission to neonatal intensive care unit
Sequence generation: 4 studies low risk; 1 study high risk; 1 study unclear risk

Allocation concealment: 1 study low risk; 5 studies unclear risk

Blinding (participants \& clinical staff): 5 studies high risk; 1 study unclear risk

Blinding (outcome assessor): 4 studies low risk; 1 study high risk; 1 study unclear risk

Incomplete outcome reporting: 5 studies low risk; 1 study unclear risk

Selective outcome reporting: 1 study low risk; 5 studies unclear risk

Other potential threats to validity: 3 studies low risk; 3 studies unclear risk

\begin{tabular}{|c|c|c|c|c|c|c|}
\hline $\begin{array}{l}\text { Transcu- } \\
\text { taneous } \\
\text { electri- } \\
\text { cal nerve } \\
\text { stimu- } \\
\text { lation } \\
\text { (TENS) for } \\
\text { pain man- } \\
\text { agement } \\
\text { in labour } \\
\text { (Dowswell } \\
\text { 2009) }\end{array}$ & $\begin{array}{l}30 \text { April } \\
2011\end{array}$ & $\begin{array}{l}17 \text { studies } \\
\text { in } 15 \text { coun- } \\
\text { tries (USA, } \\
\text { Canada, } \\
\text { UK, Ireland, } \\
\text { France, } \\
\text { Sweden, } \\
\text { Denmark, } \\
\text { Germany, } \\
\text { Norway, } \\
\text { the Nether- } \\
\text { lands, In- } \\
\text { dia, Tai- } \\
\text { wan, China, } \\
\text { Brazil, Aus- } \\
\text { tralia) be- } \\
\text { tween } 1978 \\
\text { and } 2010\end{array}$ & $\begin{array}{l}\text { Women } \\
\text { in labour } \\
\text { (risk not } \\
\text { stated) }\end{array}$ & $\begin{array}{l}\text { TENS versus place- } \\
\text { bo or usual care } \\
\text { (17 studies, } 1455 \\
\text { women) } \\
\text { TENS versus sterile } \\
\text { water injection (1 } \\
\text { study, } 22 \text { women) }\end{array}$ & $\begin{array}{l}\text { - Pain intensi- } \\
\text { ty } \\
\text { - Satisfaction } \\
\text { with pain re- } \\
\text { lief } \\
\text { - Assisted } \\
\text { vaginal birth } \\
\text { - Caesarean } \\
\text { section } \\
\text { - Side effects } \\
\text { for baby }\end{array}$ & $\begin{array}{l}\text { Sequence generation: } 3 \text { stud- } \\
\text { ies low risk; } 1 \text { study high risk; } 14 \\
\text { studies unclear risk } \\
\text { Allocation concealment: } 3 \text { stud- } \\
\text { ies low risk; } 1 \text { study high risk; } 14 \\
\text { studies unclear risk } \\
\text { Blinding (participants): } 8 \text { stud- } \\
\text { ies high risk; } 10 \text { studies unclear } \\
\text { risk } \\
\text { Blinding (clinical staff): } 8 \text { stud- } \\
\text { ies high risk; } 10 \text { studies unclear } \\
\text { risk } \\
\text { Blinding (outcome assessor): } 7 \\
\text { studies high risk; } 11 \text { studies un- } \\
\text { clear risk }\end{array}$ \\
\hline
\end{tabular}

Pain management for women in labour: an overview of systematic reviews (Review) 
Table 2. Characteristics of included Cochrane systematic reviews - non-pharmacological interventions (Continued)

(1466 women)
Incomplete outcome reporting: 10 studies low risk; 2 studies high risk; 6 studies unclear risk

Other potential threats to validity: 18 studies unclear risk

N.B. 17 studies contributed data to the review, but 18 studies were included and assessed for risk of bias

Table 3. Characteristics of included Cochrane systematic reviews - pharmacological interventions

$\begin{array}{lllllll}\begin{array}{l}\text { Review } \\ \text { title }\end{array} & \begin{array}{l}\text { Date of } \\ \text { search }\end{array} & \begin{array}{l}\text { No. Stud- } \\ \text { ies in- } \\ \text { cluded }\end{array} & \begin{array}{l}\text { Inclu- } \\ \text { sion cri- } \\ \text { teria for }\end{array} & \begin{array}{l}\text { Comparison inter- } \\ \text { ventions (no. stud- } \\ \text { ies) }\end{array} & \begin{array}{l}\text { Outcomes for which da- } \\ \text { ta were reported that } \\ \text { could be included in an } \\ \text { analysis }\end{array} & \begin{array}{l}\text { Sumary of quality of } \\ \text { evidence in reviews (risk } \\ \text { of bias) }\end{array} \\ & & \begin{array}{l}\text { (No. Pa- } \\ \text { tients in } \\ \text { included } \\ \text { studies) }\end{array} & \begin{array}{l}\text { of partici- } \\ \text { pants" }\end{array} & & & \\ & & & \end{array}$

\begin{tabular}{|c|c|c|c|c|c|c|}
\hline $\begin{array}{l}\text { Inhaled } \\
\text { analgesia } \\
\text { for pain } \\
\text { manage- } \\
\text { ment in } \\
\text { labour } \\
\text { (Klomp } \\
\text { 2012) }\end{array}$ & $\begin{array}{l}7 \text { Septem- } \\
\text { ber } \\
2011\end{array}$ & $\begin{array}{l}26 \text { studies } \\
\text { in } 8 \text { coun- } \\
\text { tries (USA, } \\
\text { Canada, } \\
\text { UK, Swe- } \\
\text { den, Nor- } \\
\text { way, Chi- } \\
\text { na, Singa- } \\
\text { pore, Iran) } \\
\text { between } \\
1969 \text { and } \\
2009 \\
\text { (2967 } \\
\text { women) }\end{array}$ & $\begin{array}{l}\text { Women } \\
\text { in labour, } \\
\text { including } \\
\text { high risk } \\
\text { groups } \\
\text { e.g. } \\
\text { preterm } \\
\text { labour or } \\
\text { following } \\
\text { induction }\end{array}$ & $\begin{array}{l}\text { Inhaled analgesia } \\
\text { versus placebo con- } \\
\text { trol/no treatment } \\
\text { (9 studies, } 1495 \\
\text { women) } \\
\text { Inhaled analge- } \\
\text { sia versus a differ- } \\
\text { ent type of inhaled } \\
\text { analgesia (14 stud- } \\
\text { ies, } 752 \text { women) } \\
\text { Inhaled analge- } \\
\text { sia of one strength } \\
\text { versus a different } \\
\text { strength ( } 2 \text { studies, } \\
625 \text { women) } \\
\text { Inhaled analgesia } \\
\text { using one type of } \\
\text { delivery system ver- } \\
\text { sus a different sys- } \\
\text { tem ( } 2 \text { studies, } 75 \\
\text { women) } \\
\text { Inhaled analge- } \\
\text { sia versus TENS (1 } \\
\text { study, } 20 \text { women) }\end{array}$ & $\begin{array}{l}\text { - Pain Intensity } \\
\text { - Satisfaction with pain } \\
\text { relief } \\
\text { - Assisted vaginal birth } \\
\text { - Caesarean section } \\
\text { - Adverse effects for } \\
\text { women (nausea; vom- } \\
\text { iting; drowsiness; } \\
\text { dizziness; blood loss; } \\
\text { pre-eclampsia) } \\
\text { - Adverse effects for } \\
\text { infants (hypoxaemia; } \\
\text { neuro-behavioural } \\
\text { score; neonatal as- } \\
\text { phyxia) } \\
\text { - Admission to special } \\
\text { care baby unit } \\
\text { - Apgar score less than } \\
\text { seven at five minutes }\end{array}$ & $\begin{array}{l}\text { Sequence generation: } 7 \\
\text { studies low risk; } 19 \text { studies } \\
\text { unclear risk } \\
\text { Allocation concealment: } \\
3 \text { studies low risk; } 23 \text { stud- } \\
\text { ies unclear risk } \\
\text { Blinding (participants } \\
\text { \& clinical staff): } 3 \text { stud- } \\
\text { ies low risk; } 4 \text { studies high } \\
\text { risk; } 19 \text { studies unclear } \\
\text { risk } \\
\text { Blinding (outcome asses- } \\
\text { sor): } 3 \text { studies low risk; } 4 \\
\text { studies high risk; } 19 \text { stud- } \\
\text { ies unclear risk } \\
\text { Incomplete outcome re- } \\
\text { porting: } 13 \text { studies low } \\
\text { risk; } 4 \text { studies high risk; } 9 \\
\text { studies unclear risk } \\
\text { Selective outcome re- } \\
\text { porting: } 16 \text { studies low } \\
\text { risk; } 5 \text { studies high risk; } 5 \\
\text { studies unclear risk } \\
\text { Other potential threats } \\
\text { to validity: } 6 \text { studies low } \\
\text { risk; } 3 \text { studies high risk; } 17 \\
\text { studies unclear risk }\end{array}$ \\
\hline $\begin{array}{l}\text { Parenter- } \\
\text { al opioids }\end{array}$ & 30 April & $\begin{array}{l}57 \text { stud- } \\
\text { ies in } 21\end{array}$ & $\begin{array}{l}\text { Women } \\
\text { in labour. }\end{array}$ & $\begin{array}{l}\text { Intramuscular (IM) } \\
\text { opioid comparisons }\end{array}$ & - Pain Intensity & $\begin{array}{l}\text { Sequence generation: } 13 \\
\text { studies low risk; } 1 \text { study }\end{array}$ \\
\hline
\end{tabular}


Table 3. Characteristics of included Cochrane systematic reviews - pharmacological interventions (Continued)

nal pain

manage-

ment in

labour

(Ullman

2010) tries (USA, focusing 16 comparisons (37

Cana-

da, UK,

Italy, Aus-

tria, Swe-

den, Nor-

way, Den-

mak, Ger-

many, the

Nether-

lands,

Turkey,

Argentina,

Brazil, In-

dia, Chi-

na, Hong

Kong, Sin-

gapore,

Thailand,

South

Africa,

Egypt,

Iran) be-

tween

1958 and

2010

(7000

women)

\section{specifi- studies)}

cally and

exclu-

sively on

women in

high-risk

groups,

or women 7 comparisons ( 10

or women studies)

mature

labour

(before

37 weeks'

gesta-

tion) were

exclud-

ed. Stud-

ies which

include

such

women

as part of

a broad-

er sample

were in-

cluded.
Intravenous (IV)

opioid comparisons

Intravenous patient

controlled opioids

(IV PCA)

5 comparisons ( 7

studies)

Opioids versus transcutaneous electrical nerve stimulation(TENS)

1 comparison ( 3

studies)
- Satisfaction with pain relief

- Satisfaction with childbirth experience

- Breastfeeding problems

- Assisted vaginal birth

- Caesarean section

- Adverse effects for women (nausea \& vomiting; sleepiness; drowsiness; blood loss)

- Adverse effects for infants (resuscitation; fetal heart rate changes; respiratory distress; neonatal neuro-behavioural score; ventilator support)

- Admission to special care baby unit

- Apgar score less than seven at five minutes high risk; 43 studies un-

clear risk

Allocation concealment:

17 studies low risk; 40

studies unclear risk

Blinding (participants):

22 studies low risk; 6 studies high risk; 29 studies un-

clear risk

Blinding (clinical staff): 22 studies low risk; 6 studies high risk; 29 studies unclear risk

Blinding (outcome assessor): 17 study low risk; 5 studies high risk; 35 studies unclear risk

Incomplete outcome reporting: 21 studies low risk; 19 studies high risk; 17 studies unclear risk

Selective outcome reporting: 1 studies low risk; 3 studies high risk; 53 studies unclear risk

Other potential threats to validity: 11 studies low risk; 2 studies high risk; 44 studies unclear risk

\begin{tabular}{|c|c|c|c|c|c|}
\hline $\begin{array}{l}\text { Non-opi- } \\
\text { oid drugs } \\
\text { for pain } \\
\text { manage- } \\
\text { ment in } \\
\text { labour } \\
\text { (Othman } \\
\text { 2012) }\end{array}$ & $\begin{array}{l}19 \text { May } \\
2011\end{array}$ & $\begin{array}{l}18 \text { studies } \\
\text { in } 7 \text { coun- } \\
\text { tries (USA, } \\
\text { Canada, } \\
\text { UK, Swe- } \\
\text { den, Ar- } \\
\text { gentina, } \\
\text { India, Chi- } \\
\text { na) be- } \\
\text { tween } \\
1963 \text { and } \\
2004 \\
\text { (2733 } \\
\text { women) }\end{array}$ & $\begin{array}{l}\text { Women } \\
\text { in labour, } \\
\text { including } \\
\text { high risk } \\
\text { groups } \\
\text { e.g. } \\
\text { preterm } \\
\text { labour or } \\
\text { following } \\
\text { induction }\end{array}$ & $\begin{array}{l}\text { Non-opioid drug } \\
\text { versus placebo } \\
\text { or no treatment } \\
\text { (14 studies, } 2003 \\
\text { women) } \\
\text { Non-opioid drug } \\
\text { versus any other } \\
\text { pain management } \\
\text { intervention e.g. } \\
\text { opioids (3 studies, } \\
563 \text { women) } \\
\text { Non-opioid drug } \\
\text { versus different } \\
\text { non-opioid drug } \\
\text { (2 studies, } 562 \\
\text { women) } \\
\text { Non-opioid drug } \\
\text { versus same non- } \\
\text { opioid different } \\
\text { dose (1 study, } 28 \\
\text { women) }\end{array}$ & $\begin{array}{l}\text { - } \text { Pain intensity } \\
\text { - Satisfaction with pain } \\
\text { relief } \\
\text { - Satisfaction with } \\
\text { childbirth experience } \\
\text { - Breastfeeding } \\
\text { - Assisted vaginal birth } \\
\text { - Caesarean section } \\
\text { - Adverse effects for } \\
\text { women } \\
\text { - Adverse effects for in- } \\
\text { fants } \\
\text { - Apgar score less than } \\
\text { seven at five minutes }\end{array}$ \\
\hline
\end{tabular}

Sequence generation: 6

studies low risk; 12 studies unclear risk

Allocation concealment: 5 studies low risk; 13 studies unclear risk

Blinding (participants/clinical staff): 14 studies low risk; 4 studies unclear risk

Blinding (outcome assessor): 3 studies low risk; 15 studies unclear risk

Incomplete outcome reporting: 16 studies low risk; 2 studies high risk

Selective outcome reporting: 12 studies low 
Table 3. Characteristics of included Cochrane systematic reviews - pharmacological interventions (Continued)

(3 studies have

more than two

arms and are in-

cluded in more

than one compari-

son group) risk; 5 studies high risk; 1

study unclear risk

Other potential threats

to validity: 13 studies low risk; 1 study high risk; 4

studies unclear risk

\begin{tabular}{|c|c|c|c|c|c|}
\hline $\begin{array}{l}\text { Local } \\
\text { anaes- } \\
\text { thetic } \\
\text { nerve } \\
\text { block for } \\
\text { pain man- } \\
\text { agement } \\
\text { in labour } \\
\text { (Novikova } \\
\text { 2012) }\end{array}$ & $\begin{array}{l}6 \text { June } \\
2011\end{array}$ & $\begin{array}{l}12 \text { studies } \\
\text { (countries } \\
\text { not stated } \\
\text { in review) } \\
\text { between } \\
1969 \text { and } \\
2009 \\
\text { (1549 } \\
\text { women) }\end{array}$ & $\begin{array}{l}\text { Women } \\
\text { in labour, } \\
\text { including } \\
\text { high risk } \\
\text { groups } \\
\text { e.g. } \\
\text { preterm } \\
\text { labour or } \\
\text { following } \\
\text { induction }\end{array}$ & $\begin{array}{l}\text { Local anaesthet- } \\
\text { ic nerve block ver- } \\
\text { sus different dose/ } \\
\text { agent or timing of } \\
\text { anaesthetic nerve } \\
\text { block ( } 8 \text { studies, } \\
1120 \text { women) } \\
\text { Local anaesthetic } \\
\text { nerve block versus } \\
\text { placebo (1 study, } \\
200 \text { women) } \\
\text { Local anaesthetic } \\
\text { nerve block versus } \\
\text { opioid ( } 2 \text { studies, } \\
129 \text { women) } \\
\text { Local anaesthetic } \\
\text { nerve block versus } \\
\text { non-opioid (1 study, } \\
100 \text { women) }\end{array}$ & $\begin{array}{l}\text { - Satisfaction with pain } \\
\text { relief } \\
\text { - Assisted vaginal birth } \\
\text { - Caesarean section } \\
\text { - Adverse effects for } \\
\text { women } \\
\text { - Apgar score less than } \\
\text { seven at five minutes }\end{array}$ \\
\hline
\end{tabular}

Sequence generation: 2 studies low risk; 2 studies high risk; 8 studies unclear risk

Allocation concealment: 1 study high risk; 11 studies unclear risk

Blinding (participants/clinical staff): 4 studies low risk; 4 studies high risk; 4 studies unclear risk

Blinding (outcome assessor): 4 studies low risk; 5 studies high risk; 3 studies unclear risk

Incomplete outcome reporting: 6 studies low risk; 4 studies high risk; 2 studies unclear risk

Selective outcome reporting: 5 studies low risk; 7 studies high risk

Other potential threats to validity: 9 studies low risk; 3 studies high risk

\begin{tabular}{|c|c|c|c|c|c|c|}
\hline $\begin{array}{l}\text { Epidur- } \\
\text { al ver- } \\
\text { sus non- } \\
\text { epidur- } \\
\text { al or no } \\
\text { analgesia } \\
\text { in labour } \\
\text { (Anim-So- } \\
\text { muah } \\
\text { 2011) }\end{array}$ & $\begin{array}{l}31 \text { March } \\
2011\end{array}$ & $\begin{array}{l}38 \text { stud- } \\
\text { ies in } 18 \\
\text { coun- } \\
\text { tries (USA, } \\
\text { Cana- } \\
\text { da, UK, } \\
\text { France, } \\
\text { Sweden, } \\
\text { Finland, } \\
\text { Denmark, } \\
\text { Mexico, } \\
\text { Russia, } \\
\text { Saudi Ara- } \\
\text { bia, Egypt, } \\
\text { Israel, } \\
\text { Iran, In- } \\
\text { dia, Tai- } \\
\text { wan, Thai- } \\
\text { land, Chi- } \\
\text { na, Aus- } \\
\text { tralia) be- }\end{array}$ & $\begin{array}{l}\text { Pregnant } \\
\text { women } \\
\text { in labour } \\
\text { request- } \\
\text { ing pain } \\
\text { relief, re- } \\
\text { gardless } \\
\text { of par- } \\
\text { ity and } \\
\text { whether } \\
\text { labour } \\
\text { was spon- } \\
\text { taneous } \\
\text { or in- } \\
\text { duced }\end{array}$ & $\begin{array}{l}\text { Epidural versus opi- } \\
\text { oids ( } 33 \text { studies, } \\
8868 \text { women) } \\
\text { Epidural versus no } \\
\text { analgesia ( } 5 \text { stud- } \\
\text { ies, } 790 \text { women) }\end{array}$ & $\begin{array}{l}\text { - Pain intensity } \\
\text { - Satisfaction with pain } \\
\text { relief } \\
\text { - Sense of control in } \\
\text { labour } \\
\text { - Satisfaction with } \\
\text { childbirth experience } \\
\text { - Assisted vaginal birth } \\
\text { - Caesarean section } \\
\text { - Adverse effects for } \\
\text { women (long-term } \\
\text { backache; maternal } \\
\text { hypotension; postna- } \\
\text { tal depression; motor } \\
\text { blockade; headache; } \\
\text { nausea and vomiting; } \\
\text { itching; fever; shiver- } \\
\text { ing; drowsiness; uri- } \\
\text { nary retention; mal- }\end{array}$ & $\begin{array}{l}\text { Sequence generation: } 18 \\
\text { studies low risk; } 1 \text { study } \\
\text { high risk; } 19 \text { studies un- } \\
\text { clear risk } \\
\text { Allocation concealment: } \\
16 \text { studies low risk; } 22 \\
\text { studies unclear risk } \\
\text { Blinding (participants): } \\
4 \text { studies low risk; } 6 \text { stud- } \\
\text { ies high risk; } 28 \text { studies un- } \\
\text { clear risk } \\
\text { Blinding (clinical staff): } \\
4 \text { studies low risk; } 3 \text { stud- } \\
\text { ies high risk; } 31 \text { studies un- } \\
\text { clear risk } \\
\text { Blinding (outcome asses- } \\
\text { sor): } 1 \text { study low risk; } 1\end{array}$ \\
\hline
\end{tabular}

Pain management for women in labour: an overview of systematic reviews (Review)

Copyright $\odot 2013$ The Cochrane Collaboration. Published by John Wiley \& Sons, Ltd. 
Table 3. Characteristics of included Cochrane systematic reviews - pharmacological interventions (Continued)

tween
1974 and
2010
(9658
women)

women) position; surgical amniotomy)

- Adverse effects for infants (acidosis; naloxone administration; meconium staining of liquor)

- Admission to special care baby unit

- Apgar score of less than seven at five minutes study high risk; 36 studies unclear risk

Incomplete outcome reporting: 12 studies low risk; 7 studies high risk; 19 studies unclear risk

Selective outcome reporting: 14 study low risk; 15 studies high risk; 9 studies unclear risk

Other potential threats to validity: 18 studies low risk; 8 studies high risk; 12 studies unclear risk

Sequence generation: 11

studies low risk; 16 studies unclear risk

- Satisfaction with pain relief

tries (USA, combined

Cana- spinaltional epidural

CSE versus low-

da, UK, epidural dose epidural

- Assisted vaginal birth

- Caesarean section

- Adverse effects for women and infants

Italy, Bel- al analge-

guim, sia com-

Spain, menced

Saudi Ara- during the

- Admission to special care baby unit/neonatal intensive care unit

bia, Brazil) first stage

between of labour

1991 and

2009

- Apgar score less than seven at five minutes

\section{Allocation concealment:}

14 studies low risk; 13

studies unclear risk

Blinding (participants/staff/assessors): 15 studies low risk; 3 studies high risk; 9 studies unclear risk

\section{Incomplete outcome re-} porting: 25 studies low risk; 1 study high risk; 1 study unclear risk

Selective outcome reporting: 23 studies low risk; 4 studies unclear risk

Other potential threats to validity: 3 studies low risk; 4 studies unclear risk (20 studies not assessed)

Table 4. Characteristics of included non-Cochrane systematic reviews - non-pharmacological

\begin{tabular}{|c|c|c|c|c|c|c|}
\hline Review title & $\begin{array}{l}\text { Date of } \\
\text { search }\end{array}$ & $\begin{array}{l}\text { No. stud- } \\
\text { ies in- } \\
\text { cluded }\end{array}$ & $\begin{array}{l}\text { Inclusion criteria } \\
\text { for "Types of par- } \\
\text { ticipants" }\end{array}$ & $\begin{array}{l}\text { Comparison interventions } \\
\text { (no. studies) }\end{array}$ & $\begin{array}{l}\text { Outcomes } \\
\text { for which } \\
\text { data were } \\
\text { reported } \\
\text { that could } \\
\text { be included } \\
\text { in an analy- } \\
\text { sis }\end{array}$ & $\begin{array}{l}\text { Summary } \\
\text { of quality } \\
\text { of evidence } \\
\text { in reviews } \\
\text { (risk of bias) }\end{array}$ \\
\hline
\end{tabular}


Table 4. Characteristics of included non-Cochrane systematic reviews - non-pharmacological (Continued)

\begin{tabular}{|c|c|c|c|}
\hline $\begin{array}{l}\text { Sterile water in- } \\
\text { jection for labour } \\
\text { pain: a system- } \\
\text { atic review and } \\
\text { meta-analysis of } \\
\text { randomised con- }\end{array}$ & $\begin{array}{l}\text { Up to } \\
2009\end{array}$ & $\begin{array}{l}8 \text { (783 } \\
\text { women) }\end{array}$ & $\begin{array}{l}\text { Pregnant women } \\
\text { who had lower back } \\
\text { pain during the ac- } \\
\text { tive stage of labour } \\
\text { and requested pain } \\
\text { medication. }\end{array}$ \\
\hline
\end{tabular}

trolled trials

(Hutton 2009)

Intracutaneous sterile or sub-
cutaneous sterile water in-
jection versus placebo con-
trol (saline) or another non-
pharmacological modality
(acupuncture; TENs) (8 stud-
ies, 783 women)

- Pain in-

Caesare- assessed usan section ing the modified Jadad scale.

Score of 10 or more $=$ considered high quality

score 8: 1 study

score 10: 4

studies

score 12: 2

studies

score 13: 1

study

Table 5. Characteristics of included non-Cochrane systematic reviews - pharmacological

\begin{tabular}{lllllll}
\hline Review title & $\begin{array}{l}\text { Date of } \\
\text { search }\end{array}$ & $\begin{array}{l}\text { No. stud- } \\
\text { ies in- } \\
\text { cluded }\end{array}$ & $\begin{array}{l}\text { Inclu- } \\
\text { sion cri- } \\
\text { teria for } \\
\text { "Types } \\
\text { of partici- } \\
\text { pants" }\end{array}$ & $\begin{array}{l}\text { Comparison } \\
\text { interventions } \\
\text { (no. studies) }\end{array}$ & $\begin{array}{l}\text { Outcomes for which data were re- } \\
\text { ported that could be included in an } \\
\text { analysis }\end{array}$ & $\begin{array}{l}\text { Summary of } \\
\text { quality of ev- } \\
\text { idence in re- } \\
\text { views (risk of } \\
\text { bias) }\end{array}$ \\
\\
\end{tabular}

\begin{tabular}{|c|c|c|c|c|c|}
\hline $\begin{array}{l}\text { Epidural } \\
\text { ropivacaine } \\
\text { versus bupi- } \\
\text { vacaine } \\
\text { for labor: a } \\
\text { meta-analy- } \\
\text { sis } \\
\text { (Halpern } \\
\text { 2003a) }\end{array}$ & $\begin{array}{l}15 \text { March } \\
2002\end{array}$ & $\begin{array}{l}23 \text { studies } \\
\text { (2074 } \\
\text { women) }\end{array}$ & $\begin{array}{l}\text { Labouring } \\
\text { women }\end{array}$ & $\begin{array}{l}\text { Ropivacaine } \\
\text { versus bupi- } \\
\text { vacaine ( } 23 \\
\text { studies, } 2074 \\
\text { women) }\end{array}$ & $\begin{array}{l}\text { - Pain intensity (time to onset of anal- } \\
\text { gesia) } \\
\text { - Satisfaction with pain relief (num- } \\
\text { bers with "excellent analgesia") } \\
\text { - Assisted vaginal birth } \\
\text { - Caesarean section } \\
\text { - Side effects for mother (hypoten- } \\
\text { sion; nausea or vomiting) } \\
\text { - Apgar score less than seven at five } \\
\text { minutes }\end{array}$ \\
\hline
\end{tabular}

Assessed the quality of studies using the Jadad quality index score. This scale has a maximum of 5 points. Studie are considered high quality if the score is 3 or more.

Score of 5: 4 studies

Score of 4: 8 studies

Score of 3: 5 studies 
Table 5. Characteristics of included non-Cochrane systematic reviews - pharmacological (Continued)

Score of 2: 1

study

NA (not ap-

plicable): 4

studies

1 study un-

published da-

ta and so not

assessed

\begin{tabular}{|c|c|c|c|c|c|c|}
\hline $\begin{array}{l}\text { Fetal brady- } \\
\text { cardia due to } \\
\text { intrathecal } \\
\text { opioids for } \\
\text { labour anal- } \\
\text { gesia: a sys- } \\
\text { tematic re- } \\
\text { view } \\
\text { (Mardirosoff } \\
\text { 2002) }\end{array}$ & $\begin{array}{l}25 \text { Janu- } \\
\text { ary } 2001\end{array}$ & $\begin{array}{l}24 \text { stud- } \\
\text { ies ( } 3513 \\
\text { women) }\end{array}$ & $\begin{array}{l}\text { Labouring } \\
\text { women }\end{array}$ & $\begin{array}{l}\text { Any intrathe- } \\
\text { cal opioid with } \\
\text { or without } \\
\text { local anaes- } \\
\text { thetic ver- } \\
\text { sus any anal- } \\
\text { gesic regimen } \\
\text { that exclud- } \\
\text { ed intrathe- } \\
\text { cal opioids ( } 24 \\
\text { studies, } 3513 \\
\text { women) }\end{array}$ & $\begin{array}{l}\text { - } \text { Assisted vaginal birth } \\
\text { - } \text { Casaerean section } \\
\text { - Side effects for mother (pruritus) } \\
\text { - } \text { Side effects for baby (fetal heart rate } \\
\text { abnormalities; fetal bradycardia) } \\
\text { - } \text { Apgar score less than seven at five } \\
\text { minutes }\end{array}$ & $\begin{array}{l}\text { Assessed ade- } \\
\text { quacy of ran- } \\
\text { domisation; } \\
\text { blinding; and } \\
\text { description of } \\
\text { withdrawals, } \\
\text { according to a } \\
5 \text {-point Oxford } \\
\text { scale. } \\
\text { Median qual- } \\
\text { ity score was } \\
3.5 \text { (range } 1 \text { to } \\
\text { 5) }\end{array}$ \\
\hline
\end{tabular}

Table 6. Quality of evidence in included Cochrane systematic reviews - non-pharmacological interventions

\begin{tabular}{|c|c|c|c|c|c|c|}
\hline Review Title & $\begin{array}{l}\text { Sequence } \\
\text { genera- } \\
\text { tion as- } \\
\text { sessed } \\
\text { (\% stud- } \\
\text { ies low } \\
\text { risk) }\end{array}$ & 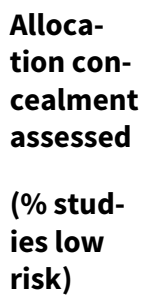 & $\begin{array}{l}\text { Blinding of participants, } \\
\text { personnel and outcome } \\
\text { assessors assessed } \\
\text { (\% studies low risk) }\end{array}$ & $\begin{array}{l}\text { Incom- } \\
\text { plete out- } \\
\text { come data } \\
\text { assessed } \\
\text { (\% studies } \\
\text { low risk) }\end{array}$ & 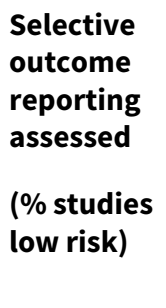 & $\begin{array}{l}\text { Other po- } \\
\text { tential } \\
\text { threats to } \\
\text { validity as- } \\
\text { sessed } \\
\text { (\% studies } \\
\text { low risk) }\end{array}$ \\
\hline $\begin{array}{l}\text { Hypnosis (7 studies) (Madden } \\
\text { 2012) }\end{array}$ & $\begin{array}{l}2 \text { studies } \\
(28 \%)\end{array}$ & $\begin{array}{l}1 \text { study } \\
(14 \%)\end{array}$ & $\begin{array}{l}\text { Participants \& Personnel - } 3 \\
\text { studies }(43 \%) \\
\text { Outcome assessors - } 3 \\
\text { studies }(43 \%)\end{array}$ & $\begin{array}{l}3 \text { studies } \\
(43 \%)\end{array}$ & $\begin{array}{l}3 \text { studies } \\
(43 \%)\end{array}$ & $\begin{array}{l}3 \text { studies } \\
(43 \%)\end{array}$ \\
\hline $\begin{array}{l}\text { Biofeedback (4 studies) (Bar- } \\
\text { ragán 2011) }\end{array}$ & $\begin{array}{l}1 \text { study } \\
(25 \%)\end{array}$ & $\begin{array}{l}0 \text { studies } \\
(0 \%)\end{array}$ & 0 studies $(0 \%)$ & $\begin{array}{l}2 \text { studies } \\
(50 \%)\end{array}$ & $\begin{array}{l}1 \text { study } \\
(25 \%)\end{array}$ & $\begin{array}{l}4 \text { studies } \\
(100 \%)\end{array}$ \\
\hline $\begin{array}{l}\text { Intracutaneous/subcuta- } \\
\text { neous sterile water injection } \\
\text { (7 studies) (Derry 2012) }\end{array}$ & $\begin{array}{l}4 \text { studies } \\
(57 \%)\end{array}$ & $\begin{array}{l}4 \text { studies } \\
(57 \%)\end{array}$ & $\begin{array}{l}\text { Participants \& Personnel - } 5 \\
\text { studies ( } 71 \%) \\
\text { Outcome assessors - } 7 \\
\text { studies }(100 \%)\end{array}$ & $\begin{array}{l}2 \text { studies } \\
(29 \%)\end{array}$ & $\begin{array}{l}4 \text { studies } \\
(57 \%)\end{array}$ & $\begin{array}{l}0 \text { studies } \\
(0 \%)\end{array}$ \\
\hline $\begin{array}{l}\text { Immersion in water (12 stud- } \\
\text { ies) (Cluett 2009) }\end{array}$ & $\begin{array}{l}6 \text { studies } \\
(50 \%)\end{array}$ & $\begin{array}{l}8 \text { studies } \\
(67 \%)\end{array}$ & 0 studies (0\%) & $\begin{array}{l}11 \text { studies } \\
(92 \%)\end{array}$ & $\begin{array}{l}10 \text { studies } \\
(83 \%)\end{array}$ & $\begin{array}{l}1 \text { study } \\
(8 \%)\end{array}$ \\
\hline $\begin{array}{l}\text { Aromatherapy (2 studies) } \\
\text { (Smith 2011c) }\end{array}$ & $\begin{array}{l}2 \text { studies } \\
(100 \%)\end{array}$ & $\begin{array}{l}2 \text { studies } \\
(100 \%)\end{array}$ & 1 study (50\%) & $\begin{array}{l}2 \text { studies } \\
(100 \%)\end{array}$ & $\begin{array}{l}0 \text { studies } \\
(0 \%)\end{array}$ & $\begin{array}{l}1 \text { study } \\
(50 \%)\end{array}$ \\
\hline
\end{tabular}

Pain management for women in labour: an overview of systematic reviews (Review) 
Table 6. Quality of evidence in included Cochrane systematic reviews - non-pharmacological interventions (Continued)

\begin{tabular}{|c|c|c|c|c|c|c|}
\hline $\begin{array}{l}\text { Relaxation techniques (11 } \\
\text { studies) (Smith 2011b) }\end{array}$ & $\begin{array}{l}6 \text { studies } \\
(54 \%)\end{array}$ & $\begin{array}{l}3 \text { studies } \\
(27 \%)\end{array}$ & 0 studies $(0 \%)$ & $\begin{array}{l}5 \text { studies } \\
(45 \%)\end{array}$ & $\begin{array}{l}1 \text { study } \\
(9 \%)\end{array}$ & $\begin{array}{l}5 \text { studies } \\
(45 \%)\end{array}$ \\
\hline $\begin{array}{l}\text { Acupuncture (13 studies) } \\
\text { (Smith 2011a) }\end{array}$ & $\begin{array}{l}12 \text { studies } \\
(92 \%)\end{array}$ & $\begin{array}{l}9 \text { studies } \\
(69 \%)\end{array}$ & 3 studies $(23 \%)$ & $\begin{array}{l}9 \text { studies } \\
(69 \%)\end{array}$ & $\begin{array}{l}0 \text { studies } \\
(0 \%)\end{array}$ & $\begin{array}{l}8 \text { studies } \\
(62 \%)\end{array}$ \\
\hline $\begin{array}{l}\text { Massage, reflexology and } \\
\text { other manual methods ( } 6 \\
\text { studies) (Smith 2012) }\end{array}$ & $\begin{array}{l}4 \text { studies } \\
(67 \%)\end{array}$ & $\begin{array}{l}1 \text { study } \\
(17 \%)\end{array}$ & $\begin{array}{l}\text { Participants \& Personnel - } 0 \\
\text { studies }(0 \%) \\
\text { Outcome assessors - } 4 \\
\text { studies }(67 \%)\end{array}$ & $\begin{array}{l}5 \text { studies } \\
(83 \%)\end{array}$ & $\begin{array}{l}1 \text { study } \\
(17 \%)\end{array}$ & $\begin{array}{l}3 \text { studies } \\
(50 \%)\end{array}$ \\
\hline $\begin{array}{l}\text { TENS (18 studies) (Dowswell } \\
\text { 2009) }\end{array}$ & $\begin{array}{l}3 \text { studies } \\
(17 \%)\end{array}$ & $\begin{array}{l}3 \text { studies } \\
(17 \%)\end{array}$ & 0 studies $(0 \%)$ & $\begin{array}{l}10 \text { studies } \\
(56 \%)\end{array}$ & $\begin{array}{l}\text { No - NOT } \\
\text { ASSESSED }\end{array}$ & $\begin{array}{l}0 \text { studies } \\
(0 \%)\end{array}$ \\
\hline
\end{tabular}

Table 7. Quality of evidence in included Cochrane systematic reviews - pharmacological interventions

\begin{tabular}{|c|c|c|c|c|c|c|}
\hline Review Title & $\begin{array}{l}\text { Sequence } \\
\text { genera- } \\
\text { tion as- } \\
\text { sessed } \\
\text { (\% stud- } \\
\text { ies low } \\
\text { risk) }\end{array}$ & $\begin{array}{l}\text { Alloca- } \\
\text { tion con- } \\
\text { cealment } \\
\text { assessed } \\
\text { (\% stud- } \\
\text { ies low } \\
\text { risk) }\end{array}$ & $\begin{array}{l}\text { Blinding of participants, per- } \\
\text { sonnel and outcome assessors } \\
\text { assessed } \\
\text { (\% studies low risk) }\end{array}$ & $\begin{array}{l}\text { Incom- } \\
\text { plete out- } \\
\text { come } \\
\text { data as- } \\
\text { sessed } \\
\text { (\% stud- } \\
\text { ies low } \\
\text { risk) }\end{array}$ & $\begin{array}{l}\text { Selective } \\
\text { outcome } \\
\text { reporting } \\
\text { assessed } \\
\text { (\% stud- } \\
\text { ies low } \\
\text { risk) }\end{array}$ & $\begin{array}{l}\text { Other po- } \\
\text { tential } \\
\text { threats to } \\
\text { validity as- } \\
\text { sessed } \\
\text { (\% studies } \\
\text { low risk) }\end{array}$ \\
\hline $\begin{array}{l}\text { Inhaled analgesia (26 } \\
\text { studies) (Klomp 2012) }\end{array}$ & $\begin{array}{l}7 \text { studies } \\
(27 \%)\end{array}$ & $\begin{array}{l}3 \text { studies } \\
(12 \%)\end{array}$ & $\begin{array}{l}\text { Participants/Personnel - } 3 \text { stud- } \\
\text { ies }(12 \%) \\
\text { Outcome assessors - } 3 \text { studies } \\
(12 \%)\end{array}$ & $\begin{array}{l}13 \text { studies } \\
(50 \%)\end{array}$ & $\begin{array}{l}16 \text { studies } \\
(62 \%)\end{array}$ & $\begin{array}{l}6 \text { studies } \\
(23 \%)\end{array}$ \\
\hline $\begin{array}{l}\text { Parenteral opioids for } \\
\text { maternal pain manage- } \\
\text { ment in labour ( } 57 \text { stud- } \\
\text { ies) (Ullman 2010) }\end{array}$ & $\begin{array}{l}13 \text { studies } \\
(23 \%)\end{array}$ & $\begin{array}{l}17 \text { studies } \\
(30 \%)\end{array}$ & $\begin{array}{l}\text { Participants - } 22 \text { studies (39\%) } \\
\text { Personnel - } 22 \text { studies (39\%) } \\
\text { Outcome assessors - } 17 \text { studies } \\
(30 \%)\end{array}$ & $\begin{array}{l}21 \text { studies } \\
(37 \%)\end{array}$ & $\begin{array}{l}1 \text { study } \\
(2 \%)\end{array}$ & $\begin{array}{l}11 \text { studies } \\
(19 \%)\end{array}$ \\
\hline $\begin{array}{l}\text { Non-opioid drugs ( } 18 \\
\text { studies) (Othman 2012) }\end{array}$ & $\begin{array}{l}6 \text { studies } \\
(33 \%)\end{array}$ & $\begin{array}{l}5 \text { studies } \\
(28 \%)\end{array}$ & $\begin{array}{l}\text { Participants/Personnel - } 14 \text { stud- } \\
\text { ies }(78 \%) \\
\text { Outcome assessors - } 3 \text { studies } \\
(17 \%)\end{array}$ & $\begin{array}{l}16 \text { studies } \\
(89 \%)\end{array}$ & $\begin{array}{l}12 \text { studies } \\
(67 \%)\end{array}$ & $\begin{array}{l}13 \text { studies } \\
(72 \%)\end{array}$ \\
\hline $\begin{array}{l}\text { Local anaesthetic nerve } \\
\text { blocks (12 studies) } \\
\text { (Novikova 2012) }\end{array}$ & $\begin{array}{l}2 \text { studies } \\
(17 \%)\end{array}$ & $\begin{array}{l}0 \text { studies } \\
(0 \%)\end{array}$ & $\begin{array}{l}\text { Participants/Personnel - } 4 \text { stud- } \\
\text { ies }(34 \%) \\
\text { Outcome assessors - } 4 \text { studies } \\
(34 \%)\end{array}$ & $\begin{array}{l}6 \text { studies } \\
(50 \%)\end{array}$ & $\begin{array}{l}5 \text { studies } \\
(42 \%)\end{array}$ & $\begin{array}{l}9 \text { studies } \\
(75 \%)\end{array}$ \\
\hline $\begin{array}{l}\text { Epidural ( } 38 \text { studies) (An- } \\
\text { im-Somuah 2011) }\end{array}$ & $\begin{array}{l}18 \text { studies } \\
(47 \%)\end{array}$ & $\begin{array}{l}16 \text { studies } \\
(42 \%)\end{array}$ & $\begin{array}{l}\text { Participants - } 4 \text { studies (11\%) } \\
\text { Personnel - } 4 \text { studies (11\%) } \\
\text { Outcome assessors - } 1 \text { study } \\
(3 \%)\end{array}$ & $\begin{array}{l}19 \text { studies } \\
(50 \%)\end{array}$ & $\begin{array}{l}14 \text { studies } \\
(37 \%)\end{array}$ & $\begin{array}{l}18 \text { studies } \\
(47 \%)\end{array}$ \\
\hline
\end{tabular}


Table 7. Quality of evidence in included Cochrane systematic reviews - pharmacological interventions (Continued)

Combined spinal epidur- 11 studies 14 studies 15 studies (56\%) al (27 studies) (Simmons (41\%) (52\%) 2012)

$(41 \%) \quad(52 \%)$

25 studies
$(93 \%)$

23 studies (85\%)

3 studies $(11 \%)$

N.B. 20 studies not assessed $(74 \%)$

Table 8. Quality of evidence in included non-Cochrane systematic reviews - non-pharmacological

\begin{tabular}{ll}
\hline Review Title & Summary of quality of evidence in reviews (risk of bias) \\
\hline $\begin{array}{l}\text { Sterile water injection for labour pain: a sys- } \\
\text { tematic review and meta-analysis of ran- }\end{array}$ & Methodological quality assessed using the modified Jadad scale. \\
domised controlled trials & Score of 10 or more = considered high quality \\
(Hutton 2009) & score 8: 1 study \\
& score 10: 4 studies \\
& score 12: 2 studies \\
& score 13: 1 study \\
\hline
\end{tabular}

Table 9. Quality of evidence in included non-Cochrane systematic reviews - pharmacological

\begin{tabular}{ll}
\hline Review Title & Summary of quality of evidence in reviews (risk of bias) \\
\hline $\begin{array}{ll}\text { Epidural ropivacaine versus bupi- } \\
\text { vacaine for labor: a meta-analysis }\end{array}$ & $\begin{array}{l}\text { Assessed the quality of studies using the Jadad quality index score. This scale has a maximum of } \\
\text { (Halpern 2003a) }\end{array}$ \\
& Score of 5: 4 studies \\
& Score of 4: 8 studies \\
& Score of 3: 5 studies \\
& Score of 2: 1 study \\
& NA (not applicable): 4 studies \\
& 1 study unpublished data and so not assessed \\
\hline $\begin{array}{l}\text { Fetal bradycardia due to in- } \\
\text { trathecal opioids for labour anal- } \\
\text { gesia: a systematic review }\end{array}$ & $\begin{array}{l}\text { Assessed adequacy of randomisation; blinding; and description of withdrawals, according to a 5- } \\
\text { point Oxford scale. }\end{array}$ \\
(Mardirosoff 2002) & Median quality score was 3.5 (range 1 to 5) \\
\hline
\end{tabular}




\begin{tabular}{|c|c|c|c|c|c|c|c|c|c|}
\hline AMSTAR criteria & $\begin{array}{l}\text { Hyp- } \\
\text { nosis } \\
\text { (Madden } \\
2012 \text { ) }\end{array}$ & $\begin{array}{l}\text { Biofeed- } \\
\text { back } \\
\text { (Bar- } \\
\text { ragán } \\
2011 \text { ) }\end{array}$ & $\begin{array}{l}\text { Intracuta- } \\
\text { neous/sub- } \\
\text { cutaneous } \\
\text { sterile wa- } \\
\text { ter injec- } \\
\text { tion (Derry } \\
2012 \text { ) }\end{array}$ & $\begin{array}{l}\text { Immer- } \\
\text { sion in } \\
\text { water } \\
\text { (Cluett } \\
2009 \text { ) }\end{array}$ & $\begin{array}{l}\text { Aro- } \\
\text { mather- } \\
\text { apy } \\
\text { (Smith } \\
2011 c \text { ) }\end{array}$ & $\begin{array}{l}\text { Relax- } \\
\text { ation } \\
\text { tech- } \\
\text { niques } \\
\text { (Smith } \\
\text { 2011b) }\end{array}$ & $\begin{array}{l}\text { Acupunc- } \\
\text { ture or } \\
\text { acupres- } \\
\text { sure } \\
\text { (Smith } \\
\text { 2011a) }\end{array}$ & $\begin{array}{l}\text { Massage, } \\
\text { reflexolo- } \\
\text { gy and oth- } \\
\text { er manual } \\
\text { methods } \\
\text { (Smith 2012) }\end{array}$ & $\begin{array}{l}\text { TENS } \\
\text { (Dowswell } \\
2009 \text { ) }\end{array}$ \\
\hline A priori design & Yes & Yes & Yes & Yes & Yes & Yes & Yes & Yes & Yes \\
\hline Duplicate selection \& extraction & Yes & Yes & Yes & Yes & Yes & Yes & Yes & Yes & Yes \\
\hline Comprehensive literature search & Yes & Yes & Yes & Yes & Yes & Yes & Yes & Yes & Yes \\
\hline $\begin{array}{l}\text { Searched for reports regardless of publication type or } \\
\text { language }\end{array}$ & Yes & Yes & Yes & Yes & Yes & Yes & Yes & Yes & Yes \\
\hline Excluded/Included list provided & Yes & Yes & Yes & Yes & Yes & Yes & Yes & Yes & Yes \\
\hline Characteristics of included studies provided & Yes & Yes & Yes & Yes & Yes & Yes & Yes & Yes & Yes \\
\hline $\begin{array}{l}\text { Quality assessment of included studies assessed \& } \\
\text { presented }\end{array}$ & Yes & Yes & Yes & Yes & Yes & Yes & Yes & Yes & Yes \\
\hline Quality used appropriately in formulating conclusions & Yes & Yes & Yes & Yes & Yes & Yes & Yes & Yes & Yes \\
\hline Methods used to combine studies appropriate & Yes & Yes & Yes & Yes & $\begin{array}{l}\text { Not ap- } \\
\text { plicable }\end{array}$ & Yes & Yes & Yes & Yes \\
\hline Publication bias assessed & $\begin{array}{l}\text { Not ap- } \\
\text { plicable }\end{array}$ & $\begin{array}{l}\text { Not ap- } \\
\text { plicable }\end{array}$ & $\begin{array}{l}\text { Not applica- } \\
\text { ble }\end{array}$ & $\begin{array}{l}\text { Not ap- } \\
\text { plicable }\end{array}$ & $\begin{array}{l}\text { Not ap- } \\
\text { plicable }\end{array}$ & $\begin{array}{l}\text { Not ap- } \\
\text { plicable }\end{array}$ & $\begin{array}{l}\text { Not ap- } \\
\text { plicable }\end{array}$ & $\begin{array}{l}\text { Not applica- } \\
\text { ble }\end{array}$ & $\begin{array}{l}\text { Not ap- } \\
\text { plicable }\end{array}$ \\
\hline Conflict of interest stated & Yes & Yes & Yes & Yes & Yes & Yes & Yes & Yes & Yes \\
\hline Total score (out of a maximum of 11 ) & 10 & 10 & 10 & 10 & 9 & 10 & 10 & 10 & 10 \\
\hline
\end{tabular}


Table 11. AMSTAR ratings for each Cochrane systematic review - pharmacological interventions

\begin{tabular}{|c|c|c|c|c|c|c|}
\hline AMSTAR criteria & $\begin{array}{l}\text { Inhaled } \\
\text { analge- } \\
\text { sia (Klomp } \\
\text { 2012) }\end{array}$ & $\begin{array}{l}\text { Opioids } \\
\text { (Ullman } \\
2010)\end{array}$ & $\begin{array}{l}\text { Non-opioid } \\
\text { drugs (Oth- } \\
\text { man 2012) }\end{array}$ & $\begin{array}{l}\text { Local anaes- } \\
\text { thetic nerve } \\
\text { blocks } \\
\text { (Novikova } \\
\text { 2012) }\end{array}$ & $\begin{array}{l}\text { Epidural } \\
\text { (Anim-So- } \\
\text { muah } \\
2011 \text { ) }\end{array}$ & $\begin{array}{l}\text { Com- } \\
\text { bined } \\
\text { spinal } \\
\text { epidural } \\
\text { (Simmons } \\
2012 \text { ) }\end{array}$ \\
\hline A priori design & Yes & Yes & Yes & Yes & Yes & Yes \\
\hline Duplicate selection \& extraction & Yes & Yes & Yes & Yes & Yes & Yes \\
\hline Comprehensive literature search & Yes & Yes & Yes & Yes & Yes & Yes \\
\hline $\begin{array}{l}\text { Searched for reports regardless of publi- } \\
\text { cation type or language }\end{array}$ & Yes & Yes & Yes & Yes & Yes & Yes \\
\hline Excluded/Included list provided & Yes & Yes & Yes & Yes & Yes & Yes \\
\hline $\begin{array}{l}\text { Characteristics of included studies pro- } \\
\text { vided }\end{array}$ & Yes & Yes & Yes & Yes & Yes & Yes \\
\hline $\begin{array}{l}\text { Quality assessment of included studies } \\
\text { assessed \& presented }\end{array}$ & Yes & Yes & Yes & Yes & Yes & Yes \\
\hline $\begin{array}{l}\text { Quality used appropriately in formulat- } \\
\text { ing conclusions }\end{array}$ & Yes & Yes & Yes & Yes & Yes & Yes \\
\hline $\begin{array}{l}\text { Methods used to combine studies ap- } \\
\text { propriate }\end{array}$ & Yes & Yes & Yes & Yes & Yes & Yes \\
\hline Publication bias assessed & $\begin{array}{l}\text { Not applic- } \\
\text { able (too } \\
\text { few stud- } \\
\text { ies for each } \\
\text { outcome } \\
\text { within differ- } \\
\text { ent compar- } \\
\text { isons) }\end{array}$ & $\begin{array}{l}\text { Not applic- } \\
\text { able (too } \\
\text { few stud- } \\
\text { ies for each } \\
\text { outcome } \\
\text { within dif- } \\
\text { ferent com- } \\
\text { parisons) }\end{array}$ & $\begin{array}{l}\text { Not applic- } \\
\text { able (too } \\
\text { few stud- } \\
\text { ies for each } \\
\text { outcome } \\
\text { within differ- } \\
\text { ent compar- } \\
\text { isons) }\end{array}$ & $\begin{array}{l}\text { Not applicable } \\
\text { (too few stud- } \\
\text { ies for each } \\
\text { outcome with- } \\
\text { in different } \\
\text { comparisons) }\end{array}$ & No & No \\
\hline Conflict of interest stated & Yes & Yes & Yes & Yes & Yes & Yes \\
\hline Total score (out of a maximum of 11 ) & 10 & 10 & 10 & 10 & 10 & 10 \\
\hline
\end{tabular}




\begin{tabular}{|c|c|c|c|}
\hline AMSTAR criteria & $\begin{array}{l}\text { Epidural ropivacaine ver- } \\
\text { sus bupivacaine for labor: a } \\
\text { meta-analysis } \\
\text { (Halpern 2003a) }\end{array}$ & $\begin{array}{l}\text { Fetal bradycardia due to } \\
\text { intrathecal opioids for } \\
\text { labour analgesia: a sys- } \\
\text { tematic review } \\
\text { (Mardirosoff 2002) }\end{array}$ & $\begin{array}{l}\text { Sterile water injection for } \\
\text { labour pain: a systematic } \\
\text { review and meta-analysis } \\
\text { of randomised controlled } \\
\text { trials } \\
\text { (Hutton 2009) }\end{array}$ \\
\hline A priori design & Cannot tell & Cannot tell & Cannot tell \\
\hline $\begin{array}{l}\text { Duplicate selection } \\
\text { \& extraction }\end{array}$ & Yes & Yes & Yes \\
\hline $\begin{array}{l}\text { Comprehensive lit- } \\
\text { erature search }\end{array}$ & Yes & Yes & Yes \\
\hline $\begin{array}{l}\text { Searched for re- } \\
\text { ports regardless of } \\
\text { publication type or } \\
\text { language }\end{array}$ & $\begin{array}{l}\text { No (limited to English lan- } \\
\text { guage publications) }\end{array}$ & Yes & Yes \\
\hline $\begin{array}{l}\text { Excluded/Included } \\
\text { list provided }\end{array}$ & No (only included studies) & Yes & Yes \\
\hline $\begin{array}{l}\text { Characteristics of } \\
\text { included studies } \\
\text { provided }\end{array}$ & Yes & Yes & Yes \\
\hline $\begin{array}{l}\text { Quality assessment } \\
\text { of included stud- } \\
\text { ies assessed \& pre- } \\
\text { sented }\end{array}$ & Yes & Yes & Yes \\
\hline $\begin{array}{l}\text { Quality used ap- } \\
\text { propriately in for- } \\
\text { mulating conclu- } \\
\text { sions }\end{array}$ & No & Cannot tell & Cannot tell \\
\hline $\begin{array}{l}\text { Methods used to } \\
\text { combine studies } \\
\text { appropriate }\end{array}$ & Yes & Yes & Yes \\
\hline
\end{tabular}




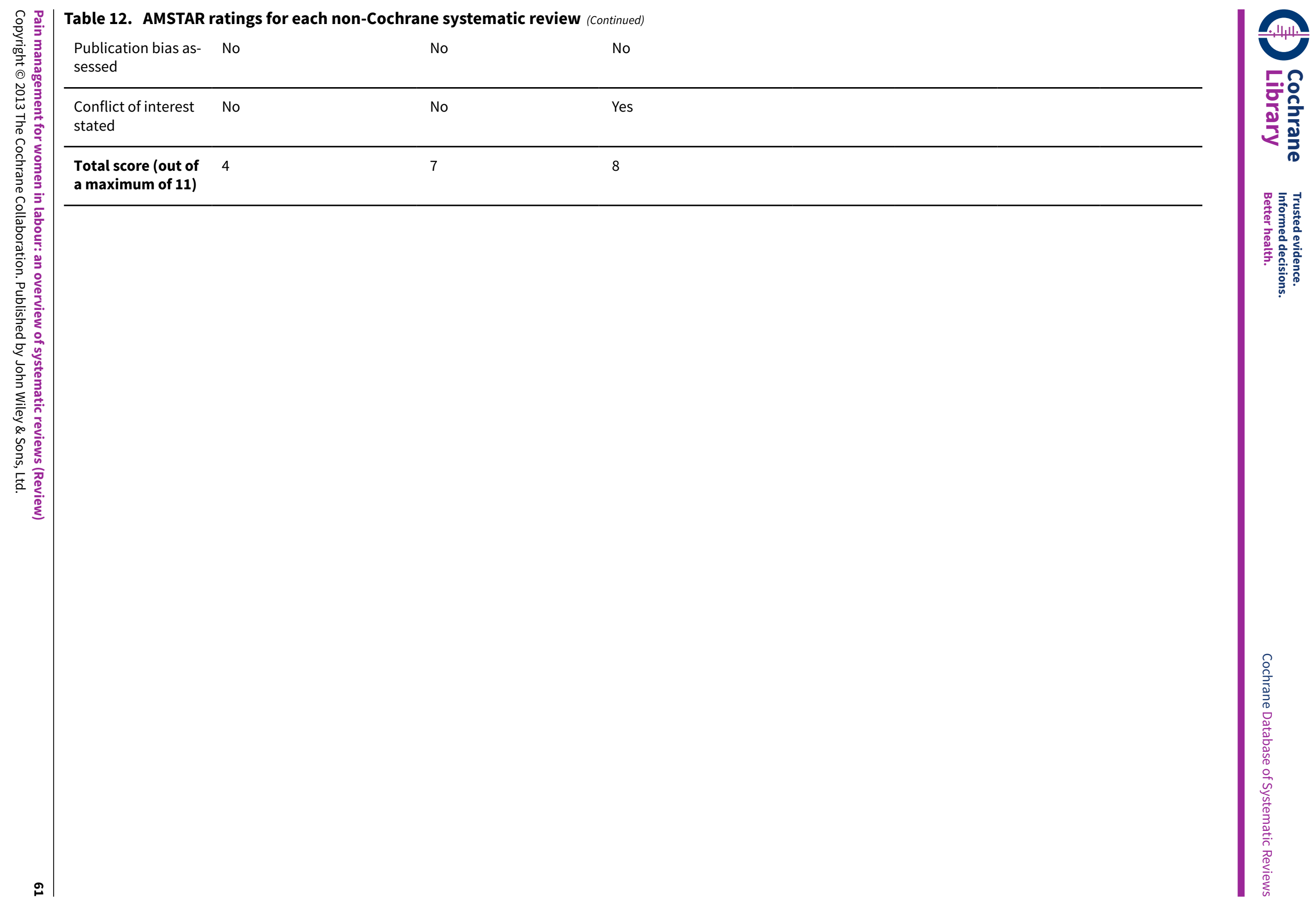


Table 13. (1.) Results by individual review - hypnosis

1. Hypnosis for pain management during labour and childbirth, 7 studies, 1213 women (Madden 2012)

1.1. Hypno- Outcome
sis versus
no hypno-
sis/standard
care (7 stud-
ies, 1070
women)

Pain intensity

\begin{tabular}{|c|c|c|}
\hline \multicolumn{2}{|l|}{ Pain intensity } & \multirow{2}{*}{$\begin{array}{l}\text { Outcome only reported for one quasi-RCT ( } 60 \\
\text { women) } \\
\begin{array}{l}\text { RR } 1.06,95 \% \mathrm{Cl} 0.94 \text { to } 1.20 \text {, no evidence of a signif- } \\
\text { icant difference }\end{array}\end{array}$} \\
\hline Satisfaction with pain relief & $1(264)$ & \\
\hline Sense of control in labour & & Outcome not reported \\
\hline Satisfaction with childbirth experience & $2(370)$ & $\begin{array}{l}\text { average } \mathrm{RR} 1.36,95 \% \mathrm{Cl} 0.52 \text { to } 3.59 \text {, no evidence of } \\
\text { a significant difference }\end{array}$ \\
\hline \multicolumn{2}{|l|}{ Effect (negative) on mother/baby interaction } & Outcome not reported \\
\hline $\begin{array}{l}\text { Breastfeeding (at discharge - any breastfeed- } \\
\text { ing) }\end{array}$ & $1(304)$ & $\begin{array}{l}\text { RR } 1.00,95 \% \mathrm{Cl} 0.97 \text { to } 1.03 \text {, no evidence of a signif- } \\
\text { icant difference }\end{array}$ \\
\hline Assisted vaginal birth & $3(414)$ & $\begin{array}{l}\text { average RR } 0.73,95 \% \mathrm{Cl} 0.36 \text { to } 1.50 \text {, no evidence of } \\
\text { a significant difference }\end{array}$ \\
\hline Caesarean section & $3(867)$ & $\begin{array}{l}\text { average RR } 0.54,95 \% \mathrm{Cl} 0.18 \text { to } 1.62 \text {, no evidence of } \\
\text { a significant difference }\end{array}$ \\
\hline $\begin{array}{l}\text { Adverse effects for women (post partum } \\
\text { haemorrhage) }\end{array}$ & $1(305)$ & $\begin{array}{l}\text { RR } 1.68,95 \% \mathrm{Cl} 0.90 \text { to } 3.12 \text {, no evidence of a signif- } \\
\text { icant difference }\end{array}$ \\
\hline $\begin{array}{l}\text { Adverse effects for women (post partum } \\
\text { blood transfusion) }\end{array}$ & $1(305)$ & $\begin{array}{l}\mathrm{RR} 3.92,95 \% \mathrm{Cl} 0.44 \text { to } 34.69 \text {, no evidence of a sig- } \\
\text { nificant difference }\end{array}$ \\
\hline $\begin{array}{l}\text { Adverse effects for women (postnatal depres- } \\
\text { sion) }\end{array}$ & $1(305)$ & $\begin{array}{l}\text { RR } 1.17,95 \% \mathrm{Cl} 0.77 \text { to } 1.78 \text {, no evidence of a signif- } \\
\text { icant difference }\end{array}$ \\
\hline $\begin{array}{l}\text { Adverse effects for women (admission to } \\
\text { HDU) }\end{array}$ & $1(305)$ & $\begin{array}{l}\text { RR } 1.47,95 \% \mathrm{Cl} 0.25 \text { to } 8.68, \text { no evidence of a signif- } \\
\text { icant difference }\end{array}$ \\
\hline $\begin{array}{l}\text { Adverse effects for women (readmission to } \\
\text { hospital) }\end{array}$ & $1(267)$ & $\begin{array}{l}\text { RR } 1.56,95 \% \mathrm{Cl} 0.62 \text { to } 3.90 \text {, no evidence of a signif- } \\
\text { icant difference }\end{array}$ \\
\hline $\begin{array}{l}\text { Adverse effects for infants (newborn resusci- } \\
\text { tation) }\end{array}$ & $1(520)$ & $\begin{array}{l}\text { RR } 0.67,95 \% \mathrm{Cl} 0.11 \text { to } 3.96 \text {, no evidence of a signif- } \\
\text { icant difference }\end{array}$ \\
\hline $\begin{array}{l}\text { Adverse effects for infants (readmission to } \\
\text { hospital) }\end{array}$ & $1(267)$ & $\begin{array}{l}\text { RR } 1.39,95 \% \mathrm{Cl} 0.64 \text { to } 3.02 \text {, no evidence of a signif- } \\
\text { icant difference }\end{array}$ \\
\hline
\end{tabular}

\section{No. of stud- Results \\ ies (no. \\ women)}


Table 13. (1.) Results by individual review - hypnosis (Continued)

\begin{tabular}{|c|c|c|c|}
\hline & $\begin{array}{l}\text { Admission to special care baby unit/neonatal } \\
\text { intensive care unit }\end{array}$ & $2(345)$ & $\begin{array}{l}\text { average RR } 0.58,95 \% \mathrm{Cl} 0.12 \text { to } 2.83 \text {, no evidence of } \\
\text { a significant difference }\end{array}$ \\
\hline & Apgar score less than seven at five minutes & $1(305)$ & $\begin{array}{l}\text { RR } 0.49,95 \% \mathrm{Cl} 0.04 \text { to } 5.35 \text {, no evidence of a signif- } \\
\text { icant difference }\end{array}$ \\
\hline & Poor infant outcomes at long-term follow-up & & Outcome not reported \\
\hline & Cost & & Outcome not reported \\
\hline \multirow{15}{*}{$\begin{array}{l}\text { 1.2. One } \\
\text { type of hyp- } \\
\text { nosis ver- } \\
\text { sus anoth- } \\
\text { er type of } \\
\text { hypnosis (1 } \\
\text { study, } 297 \\
\text { women) }\end{array}$} & Outcome & $\begin{array}{l}\text { No. of stud- } \\
\text { ies (no. } \\
\text { women) }\end{array}$ & Results \\
\hline & Pain intensity & & Outcome not reported \\
\hline & Satisfaction with pain relief & $1(267)$ & $\begin{array}{l}\text { RR } 1.17,95 \% \mathrm{Cl} 1.02 \text { to } 1.34 \text {, no evidence of a signif- } \\
\text { icant difference }\end{array}$ \\
\hline & Sense of control in labour & & Outcome not reported \\
\hline & Satisfaction with childbirth experience & $1(297)$ & $\begin{array}{l}\text { RR } 0.96,95 \% \mathrm{Cl} 0.83 \text { to } 1.10 \text {, no evidence of a signif- } \\
\text { icant difference }\end{array}$ \\
\hline & Effect (negative) on mother/baby interaction & & Outcome not reported \\
\hline & $\begin{array}{l}\text { Breastfeeding (at discharge - any breastfeed- } \\
\text { ing) }\end{array}$ & $1(296)$ & $\begin{array}{l}\text { RR } 0.99,95 \% \mathrm{Cl} 0.97 \text { to } 1.02 \text {, no evidence of a signif- } \\
\text { icant difference }\end{array}$ \\
\hline & Assisted vaginal birth & $1(297)$ & $\begin{array}{l}\text { RR } 0.85,95 \% \mathrm{Cl} 0.55 \text { to } 1.30 \text {, no evidence of a signif- } \\
\text { icant difference }\end{array}$ \\
\hline & Caesarean section & $1(297)$ & $\begin{array}{l}\text { RR } 1.41,95 \% \mathrm{Cl} 0.90 \text { to } 2.21 \text {, no evidence of a signif- } \\
\text { icant difference }\end{array}$ \\
\hline & $\begin{array}{l}\text { Adverse effects for women (post partum } \\
\text { haemorrhage) }\end{array}$ & $1(297)$ & $\begin{array}{l}\mathrm{RR} 1.01,95 \% \mathrm{Cl} 0.60 \text { to } 1.72 \text {, no evidence of a signif- } \\
\text { icant difference }\end{array}$ \\
\hline & $\begin{array}{l}\text { Adverse effects for women (post partum } \\
\text { blood transfusion) }\end{array}$ & $1(297)$ & $\begin{array}{l}\mathrm{RR} 0.53,95 \% \mathrm{Cl} 0.16 \text { to } 1.77 \text {, no evidence of a signif- } \\
\text { icant difference }\end{array}$ \\
\hline & $\begin{array}{l}\text { Adverse effects for women (postnatal depres- } \\
\text { sion) }\end{array}$ & $1(297)$ & $\begin{array}{l}\mathrm{RR} 1.43,95 \% \mathrm{Cl} 0.90 \text { to } 2.27 \text {, no evidence of a signif- } \\
\text { icant difference }\end{array}$ \\
\hline & $\begin{array}{l}\text { Adverse effects for women (admission to } \\
\text { HDU) }\end{array}$ & $1(297)$ & $\begin{array}{l}\text { RR } 0.40,95 \% \mathrm{Cl} 0.10 \text { to } 1.51 \text {, no evidence of a signif- } \\
\text { icant difference }\end{array}$ \\
\hline & $\begin{array}{l}\text { Adverse effects for women (readmission to } \\
\text { hospital) }\end{array}$ & $1(267)$ & $\begin{array}{l}\mathrm{RR} 1.36,95 \% \mathrm{Cl} 0.57 \text { to } 3.29 \text {, no evidence of a signif- } \\
\text { icant difference }\end{array}$ \\
\hline & $\begin{array}{l}\text { Adverse effects for infants (readmission to } \\
\text { hospital) }\end{array}$ & $1(267)$ & $\begin{array}{l}\text { RR } 1.54,95 \% \mathrm{Cl} 0.69 \text { to } 3.44 \text {, no evidence of a signif- } \\
\text { icant difference }\end{array}$ \\
\hline
\end{tabular}


Table 13. (1.) Results by individual review - hypnosis (Continued)

$\begin{array}{lll}\begin{array}{l}\text { Admission to special care baby unit/neonatal } \\ \text { intensive care unit }\end{array} & 1(297) & \begin{array}{l}\text { RR } 1.09,95 \% \mathrm{Cl} 0.79 \text { to } 1.51, \text { no evidence of a signif- } \\ \text { icant difference }\end{array}\end{array}$
intensive care unit icant difference

Apgar score less than seven at five minutes $\quad 1(297)$

RR $0.93,95 \% \mathrm{Cl} 0.06$ to 14.71 , no evidence of a significant difference

\begin{tabular}{ll}
\hline Poor infant outcomes at long-term follow-up & Outcome not reported \\
\hline Cost & Outcome not reported \\
\hline
\end{tabular}

$\mathrm{I}^{2}$ values reported in table only when random effects analysis has been carried out due to substantial heterogeneity indicated by an $\mathrm{I}^{2}$ value greater than $30 \%$

$\mathrm{Cl}$ : confidence interval; RCT: randomised controled trial; RR: risk ratio

Table 14. (2.) Results by individual review - biofeedback

\section{Biofeedback for pain management during labour, 4 studies, 201 women (Barragán 2011)}

\begin{tabular}{|c|c|c|c|}
\hline $\begin{array}{l}2.1 . \\
\text { Biofeed- } \\
\text { back versus } \\
\text { no biofeed- } \\
\text { back/stan- } \\
\text { dard care }\end{array}$ & Outcome & $\begin{array}{l}\text { No. of stud- } \\
\text { ies (no. } \\
\text { women) }\end{array}$ & Results \\
\hline
\end{tabular}

Pain intensity

$3(193)$
Data could not be included in analysis: no data provided for the control group in one study; data reported only in a figure in one study; no numerical data provided in one study, described narratively.

\begin{tabular}{|c|c|c|}
\hline Satisfaction with pain relief & $1(55)$ & $\begin{array}{l}\text { No data provided for the control group, so data could not } \\
\text { be analysed. }\end{array}$ \\
\hline Sense of control in labour & $1(55)$ & $\begin{array}{l}\text { No data provided for the control group, so data could not } \\
\text { be analysed. }\end{array}$ \\
\hline $\begin{array}{l}\text { Satisfaction with childbirth experi- } \\
\text { ence }\end{array}$ & & Outcome not reported \\
\hline $\begin{array}{l}\text { Effect (negative) on mother/baby in- } \\
\text { teraction }\end{array}$ & & Outcome not reported \\
\hline Breastfeeding & & Outcome not reported \\
\hline Assisted vaginal birth & $2(103)$ & $\begin{array}{l}\text { Average RR } 0.75,95 \% \mathrm{Cl} 0.18 \text { to } 3.10 \text { (random effects; het- } \\
\text { erogeneity: } \mathrm{I}^{2}=80 \%, \mathrm{Tau}^{2}=0.86, \mathrm{Chi}^{2} \text { test for heterogeneity } \\
\mathrm{P}=0.02 \text { ), no evidence of a significant difference }\end{array}$ \\
\hline Caesarean section & $2(103)$ & $\begin{array}{l}\mathrm{RR} 0.41,95 \% \mathrm{Cl} 0.14 \text { to } 1.15 \text {, no evidence of a significant dif- } \\
\text { ference }\end{array}$ \\
\hline Adverse effects & & Outcome not reported \\
\hline
\end{tabular}


Table 14. (2.) Results by individual review - biofeedback (Continued)
Admission to special care baby unit/
Outcome not reported neonatal intensive care unit
Outcome not reported

\begin{tabular}{lll}
$\begin{array}{l}\text { Admission to special care baby unit/ } \\
\text { neonatal intensive care unit }\end{array}$ & Outcome not reported \\
\hline $\begin{array}{l}\text { Apgar score less than seven at five } \\
\text { minutes }\end{array}$ & $2(103)$ & Mean values reported without standard deviations. \\
\hline $\begin{array}{l}\text { Poor infant outcomes at long-term } \\
\text { follow-up }\end{array}$ & Outcome not reported \\
\hline Cost & Outcome not reported \\
\hline
\end{tabular}

12 values reported in table only when random effects analysis has been carried out due to substantial heterogeneity indicated by an 12 value greater than $30 \%$

$\mathrm{Cl}$ : confidence interval; RR: risk ratio

Table 15. (3.) Results by individual review - sterile water

\section{Intracutaneous or subcutaneous sterile water injection compared with blinded controls for pain management in labour, 7 studies, 766 women (Derry 2012)}

\subsection{Sterile water injec- Outcome tion versus placebo \\ Outcome}

women)

\begin{tabular}{|c|c|c|c|}
\hline $\begin{array}{l}\text { Intracutaneous sterile } \\
\text { water injection versus } \\
\text { placebo }\end{array}$ & Pain intensity & & $\begin{array}{l}\text { Outcome not reported in a suitable for- } \\
\text { mat }\end{array}$ \\
\hline \multirow[t]{8}{*}{$\begin{array}{l}\text { Subcutaneous sterile } \\
\text { water injection versus } \\
\text { placebo }\end{array}$} & Pain intensity & & $\begin{array}{l}\text { Outcome not reported in a suitable for- } \\
\text { mat }\end{array}$ \\
\hline & Satisfaction with pain relief & & Outcome not reported \\
\hline & Sense of control in labour & & Outcome not reported \\
\hline & Satisfaction with childbirth experience & & Outcome not reported \\
\hline & Effect (negative) on mother/baby interaction & & Outcome not reported \\
\hline & Breastfeeding & & Outcome not reported \\
\hline & Assisted vaginal birth & $6(666)$ & $\begin{array}{l}\text { RR } 1.31,95 \% \mathrm{Cl} 0.79 \text { to } 2.18 \text {, no signifi- } \\
\text { cant difference between groups }\end{array}$ \\
\hline & Caesarean section & $7(766)$ & $\begin{array}{l}\text { RR } 0.58,95 \% \mathrm{Cl} 0.33 \text { to } 1.02 \text {, no signifi- } \\
\text { cant difference between groups }\end{array}$ \\
\hline $\begin{array}{l}\text { Intracutaneous sterile } \\
\text { water injection versus } \\
\text { placebo }\end{array}$ & $\begin{array}{l}\text { Adverse effects for women - pain at injection } \\
\text { site }\end{array}$ & $1(62)$ & Outcome data not analysed \\
\hline
\end{tabular}

\section{No. of stud- Results \\ ies (no.}

Outcome not reported in a suitable for 
Table 15. (3.) Results by individual review - sterile water (Continued)

\begin{tabular}{lll}
$\begin{array}{l}\text { Subcutaneous sterile } \\
\text { water injection versus } \\
\text { placebo }\end{array}$ & $\begin{array}{l}\text { Adverse effects for women - pain at injection } \\
\text { site }\end{array}$ & 1 (62) \\
\hline & Adverse effects for infant & Outcome not reported \\
\hline Admission to neonatal intensive care unit & Outcome not reported \\
\hline Apgar score less than seven at five minutes & Outcome not reported in this format \\
\hline Poor infant outcomes at long-term follow-up & Outcome not reported \\
\hline Cost & Outcome not reported
\end{tabular}

$\mathrm{I}^{2}$ values reported in table only when random effects analysis has been carried out due to substantial heterogeneity indicated by an $\mathrm{I}^{2}$ value greater than $30 \%$

$\mathrm{Cl}$ : confidence interval; RR: risk ratio

Table 16. (4.) Results by individual review - immersion in water

4. Immersion in water in labour and birth, 12 studies, 3252 women (Cluett 2009)

\begin{tabular}{|c|c|c|c|}
\hline $\begin{array}{l}\text { 4.1. Immer- } \\
\text { sion versus } \\
\text { no immer- } \\
\text { sion in the } \\
\text { first stage } \\
\text { of labour } \\
\text { (10 stud- } \\
\text { ies, } 2932 \\
\text { women) }\end{array}$ & Outcome & $\begin{array}{l}\text { No. of stud- } \\
\text { ies (no. } \\
\text { women) }\end{array}$ & Results \\
\hline
\end{tabular}

\begin{tabular}{lcc}
\hline $\begin{array}{l}\text { Pain intensity - mean visual analogue pain scores } \\
\text { (VAS) at the start of assessment period }\end{array}$ & 2 (141) & $\begin{array}{l}\text { MD -0.01, 95\% Cl -0.54 to 0.52, no difference } \\
\text { between groups in pain intensity }\end{array}$ \\
\hline $\begin{array}{l}\text { Pain intensity - mean visual analogue pain scores } \\
\text { (VAS) up to one hour after the start of assessment }\end{array}$ & $2(141)$ & $\begin{array}{l}\mathrm{MD}-0.81,95 \% \mathrm{Cl}-1.34 \text { to }-0.28, \text { no difference } \\
\text { between groups in pain intensity }\end{array}$ \\
\hline $\begin{array}{l}\text { Pain intensity - Ordinal description as moderate to } \\
\text { severe, 30 mins after randomisation }\end{array}$ & $1(120)$ & $\begin{array}{l}\text { RR } 0.75,95 \% \mathrm{Cl} 0.62 \text { to } 0.91, \text { significantly few- } \\
\text { er women in the immersion group experi- } \\
\text { enced moderate to severe pain }\end{array}$
\end{tabular}

Pain intensity - VAS scale 8 to 10,30 mins after ran- $1(120)$ domisation
RR $0.72,95 \% \mathrm{Cl} 0.58$ to 0.90 , significantly fewer women in the immersion group had VAS score of 8 to 10
Pain intensity - Ordinal scale pain faces 4 to 5, 30 mins after randomisation
$1(120)$

RR $0.67,95 \%$ Cl 0.51 to 0.90 , significantly fewer women in the immersion group had pain faces 4 to 5 on pain scale 
Table 16. (4.) Results by individual review - immersion in water (Continued)

Pain intensity - Ordinal description as moderate to 1 (117) severe, $1 \mathrm{hr}$ after randomisation

RR $0.76,95 \% \mathrm{Cl} 0.63$ to 0.91 , significantly fewer women in the immersion group experienced moderate to severe pain
Pain intensity - VAS scale 8 to $10,1 \mathrm{hr}$ after randomisation
$1(117)$

RR $1.21,95 \% \mathrm{Cl} 0.69$ to 2.11 , no evidence of a significant difference between groups
Pain intensity - Ordinal scale pain faces 4 to $5,1 \mathrm{hr} \quad 1(117)$ after randomisation
RR $0.68,95 \% \mathrm{Cl} 0.53$ to 0.86 , significantly fewer women in the immersion group had pain faces 4 to 5 on pain scale

\begin{tabular}{|c|c|c|}
\hline $\begin{array}{l}\text { Pain intensity - Ordinal description as moderate to } \\
\text { severe, } 2 \text { hrs after randomisation }\end{array}$ & $1(57)$ & $\begin{array}{l}0.76,95 \% \mathrm{Cl} 0.59 \text { to } 0.98 \text {, significantly fewer } \\
\text { women in the immersion group experienced } \\
\text { moderate to severe pain }\end{array}$ \\
\hline $\begin{array}{l}\text { Pain intensity - VAS scale } 8 \text { to } 10,2 \text { hrs after ran- } \\
\text { domisation }\end{array}$ & $1(57)$ & $\begin{array}{l}\mathrm{RR} 0.83,95 \% \mathrm{Cl} 0.66 \text { to } 1.05 \text {, no evidence of a } \\
\text { significant difference between groups }\end{array}$ \\
\hline $\begin{array}{l}\text { Pain intensity -Ordinal scale pain faces } 4 \text { to } 5,2 \text { hrs } \\
\text { after randomisation }\end{array}$ & $1(57)$ & $\begin{array}{l}\text { RR } 0.72,95 \% \mathrm{Cl} 0.52 \text { to } 0.98 \text {, significantly few- } \\
\text { er women in immersion group had pain faces } \\
4 \text { to } 5 \text { on pain scale }\end{array}$ \\
\hline $\begin{array}{l}\text { Pain intensity - Ordinal description as moderate to } \\
\text { severe, } 3 \text { hrs after randomisation }\end{array}$ & $1(32)$ & $\begin{array}{l}\mathrm{RR} 0.52,95 \% \mathrm{Cl} 0.23 \text { to } 1.16 \text {, no evidence of a } \\
\text { significant difference between groups }\end{array}$ \\
\hline $\begin{array}{l}\text { Pain intensity - VAS scale } 8 \text { to } 10,3 \text { hrs after ran- } \\
\text { domisation }\end{array}$ & $1(32)$ & $\begin{array}{l}\text { RR } 0.69,95 \% \mathrm{Cl} 0.39 \text { to } 1.23 \text {, no evidence of a } \\
\text { significant difference between groups }\end{array}$ \\
\hline $\begin{array}{l}\text { Pain intensity - Ordinal scale pain faces } 4 \text { to } 5,3 \mathrm{hrs} \\
\text { after randomisation }\end{array}$ & $1(32)$ & $\begin{array}{l}\text { RR } 0.57,95 \% \mathrm{Cl} 0.25 \text { to } 1.27 \text {, no evidence of a } \\
\text { significant difference between groups }\end{array}$ \\
\hline $\begin{array}{l}\text { Pain intensity - Ordinal description as moderate to } \\
\text { severe, } 24 \text { hrs after randomisation }\end{array}$ & $1(119)$ & $\begin{array}{l}\mathrm{RR} 0.64,95 \% \mathrm{Cl} 0.50 \text { to } 0.82 \text {, significantly few- } \\
\text { er women in the immersion group experi- } \\
\text { enced moderate to severe pain }\end{array}$ \\
\hline $\begin{array}{l}\text { Pain intensity - VAS scale } 8 \text { to } 10,24 \text { hrs after ran- } \\
\text { domisation }\end{array}$ & $1(119)$ & $\begin{array}{l}\text { RR } 0.62,95 \% \mathrm{Cl} 0.49 \text { to } 0.80 \text {, significantly few- } \\
\text { er women in the immersion group had VAS } \\
\text { score of } 8 \text { to } 10\end{array}$ \\
\hline $\begin{array}{l}\text { Pain intensity - Ordinal scale pain faces } 4 \text { to } 5,24 \\
\text { hrs after randomisation }\end{array}$ & $1(119)$ & $\begin{array}{l}\text { RR } 0.69,95 \% \mathrm{Cl} 0.54 \text { to } 0.87 \text {, significantly few- } \\
\text { er women in the immersion group had pain } \\
\text { faces } 4 \text { to } 5 \text { on pain scale }\end{array}$ \\
\hline Satisfaction with pain relief & & Outcome not reported \\
\hline Sense of control in labour & & Outcome not reported \\
\hline Satisfaction with childbirth experience & & Outcome not reported \\
\hline Effect on mother/baby interaction & & Outcome not reported \\
\hline
\end{tabular}


Table 16. (4.) Results by individual review - immersion in water (Continued)

\begin{tabular}{|c|c|c|}
\hline $\begin{array}{l}\text { Breastfeeding (not breastfeeding after six weeks } \\
\text { post delivery) }\end{array}$ & $2(363)$ & $\begin{array}{l}\text { RR } 1.17,95 \% \mathrm{Cl} 0.64 \text { to } 2.15 \text {, no evidence of a } \\
\text { significant difference between groups }\end{array}$ \\
\hline Assisted vaginal birth & $7(2628)$ & $\begin{array}{l}\mathrm{RR} 0.86,95 \% \mathrm{Cl} 0.71 \text { to } 1.05 \text {, no evidence of a } \\
\text { significant difference between groups }\end{array}$ \\
\hline Caesarean section & $8(2712)$ & $\begin{array}{l}\mathrm{RR} 1.21,95 \% \mathrm{Cl} 0.87 \text { to } 1.68 \text {, no evidence of a } \\
\text { significant difference between groups }\end{array}$ \\
\hline $\begin{array}{l}\text { Adverse effects for women (postpartum haemor- } \\
\text { rhage) }\end{array}$ & $1(274)$ & $\begin{array}{l}\mathrm{RR} 1.58,95 \% \mathrm{Cl} 0.80 \text { to } 3.13 \text {, no evidence of a } \\
\text { significant difference between groups }\end{array}$ \\
\hline $\begin{array}{l}\text { Adverse effects for women (blood loss - mean } \\
\text { blood loss } \mathrm{ml} \text { ) }\end{array}$ & $2(153)$ & $\begin{array}{l}\mathrm{MD}-14.33 .95 \% \mathrm{Cl}-63.03 \text { to } 34.37 \text {, no evidence } \\
\text { of a significant difference between groups }\end{array}$ \\
\hline $\begin{array}{l}\text { Adverse effects for women (perineal trauma - epi- } \\
\text { siotomy) }\end{array}$ & $5(1272)$ & $\begin{array}{l}\mathrm{RR} 0.93,95 \% \mathrm{Cl} 0.80 \text { to } 1.08 \text {, no evidence of a } \\
\text { significant difference between groups }\end{array}$ \\
\hline $\begin{array}{l}\text { Adverse effects for women (perineal trauma - sec- } \\
\text { ond-degree tear) }\end{array}$ & $5(1286)$ & $\begin{array}{l}\mathrm{RR} 0.94,95 \% \mathrm{Cl} 0.74 \text { to } 1.20 \text {, no evidence of a } \\
\text { significant difference between groups }\end{array}$ \\
\hline $\begin{array}{l}\text { Adverse effects for women (perineal trauma - third } \\
\text { or fourth degree tear) }\end{array}$ & $5(2401)$ & $\begin{array}{l}\mathrm{RR} 1.37,95 \% \mathrm{Cl} 0.86 \text { to } 2.17 \text {, no evidence of a } \\
\text { significant difference between groups }\end{array}$ \\
\hline $\begin{array}{l}\text { Adverse effects for women (systolic blood pres- } \\
\text { sure) }\end{array}$ & $1(120)$ & $\begin{array}{l}\mathrm{MD}-7.20,95 \% \mathrm{Cl}-13.12 \text { to }-1.28 \text {, mean systolic } \\
\text { blood pressure significantly lower in the im- } \\
\text { mersion group }\end{array}$ \\
\hline $\begin{array}{l}\text { Adverse effects for women (diastolic blood pres- } \\
\text { sure) }\end{array}$ & $1(120)$ & $\begin{array}{l}\text { MD }-10.20,95 \% \mathrm{Cl}-13.70 \text { to }-6.70 \text {, mean dias- } \\
\text { tolic blood pressure significantly lower in the } \\
\text { immersion group }\end{array}$ \\
\hline
\end{tabular}

Adverse effects for women (arterial blood pressure) $1(120)$

MD $-10.50,95 \% \mathrm{Cl}-14.68$ to -6.32 , mean arterial blood pressure significantly lower in the immersion group

Adverse effects for women (maternal infection - $\quad 5$ (1295) perineal, systemic, uterine or increase in temperature) RR $0.99,95 \% \mathrm{Cl} 0.50$ to 1.96 , no significant difference between groups

Adverse effects for women (postpartum depression 2 (370) - score of more than 11 on the Edinburgh Postnatal Depression Scale EPDS)

RR $1.38,95 \% \mathrm{Cl} 0.85$ to 2.24 , no significant difference between groups in the incidence of postpartum depression

Adverse effects for infants (presence of meconium $\quad 5(1260)$ stained liquor)

RR $0.95,95 \% \mathrm{Cl} 0.76$ to 1.19 , no evidence of a significant difference between groups for meconium staining

Adverse effects for infants (abnormal fetal heart $3(487)$

RR $0.75,95 \%$ Cl 0.34 to 1.67 , (random effects; rate patterns) heterogeneity: $\left.\right|^{2}=57 \%$, Tau $^{2}=0.22$, Chi $^{2}$ test for heterogeneity $\mathrm{P}=0.13$ ), no evidence of a significant difference

Adverse effects for infants (umbilical artery pH less $1(110)$ than 7.20 )
RR $5.18,95 \% \mathrm{Cl} 0.25$ to 105.51 , no evidence of a significant difference between groups 
Table 16. (4.) Results by individual review - immersion in water (Continued)

$\begin{array}{lll}\begin{array}{l}\text { Adverse effects for infants (temperature greater } \\ \text { than 37.8 degrees C as an indicator for infection) }\end{array} & 1(274) & \begin{array}{l}\text { RR } 1.00,95 \% \mathrm{Cl} 0.06 \text { to } 15.83, \text { no evidence of a } \\ \text { significant difference between groups }\end{array}\end{array}$

\begin{tabular}{ccc}
\hline Adverse effects for infants (neonatal infection) & 5 (1295) & $\begin{array}{l}\text { RR 2.00, 95\% CI 0.50 to 7.94, no evidence of a } \\
\text { significant difference between groups }\end{array}$ \\
\hline Admission to neonatal intensive care unit & $3(1571)$ & $\begin{array}{l}\text { RR 1.06, 95\% CI 0.71 to 1.57, no evidence of a } \\
\text { significant difference between groups }\end{array}$ \\
\hline Apgar score less than seven at five minutes & $5(1834)$ & $\begin{array}{l}\text { RR 1.58, 95\% Cl 0.63 to 3.93, no evidence of a } \\
\text { significant difference between groups }\end{array}$ \\
\hline Poor infant outcomes at long-term follow-up & Outcome not reported \\
\hline Cost & Outcome not reported \\
\hline
\end{tabular}

4.1. Immer- Outcome
sion ver-
sus no im-
mersion
in the sec-
ond stage
of labour (3
studies, 286
women)

\section{No. of stud- Results}

ies (no.

women)
Pain intensity - number of women reporting mod- 1 (117) erate to severe pain on an ordinal scale

Satisfaction with pain relief

Sense of control in labour

Satisfaction with childbirth experience (report-

ed that they did not cope satisfactorily with their pushing efforts)

Satisfaction with childbirth experience (satisfaction with labour and birth on scale of 0-6 where 0 is not at all satisfied)

$1(60)$
RR $1.06,95 \% \mathrm{Cl} 0.73$ to 1.53 , no significant difference between groups in women experiencing moderate to severe pain

Outcome not reported

Outcome not reported

$1(117)$

RR $0.24,95 \%$ Cl 0.07 to 0.80 , significantly fewer women in the immersion group reported low satisfaction

MD $0.03,95 \% \mathrm{Cl}-0.64$ to 0.70 , no significant difference between mean satisfaction scores

Outcome not reported

Effect (negative) on mother/baby interaction

$1(60)$

RR $0.86,95 \% \mathrm{Cl} 0.69$ to 1.08 , no evidence of a significant difference between groups

Assisted vaginal birth

$2(180)$

RR $0.73,95 \% \mathrm{Cl} 0.21$ to 2.54 , no evidence of a significant difference between groups

Caesarean section

$2(180)$

$2(179)$

Adverse effects for women (perineal trauma - episiotomy)
RR $0.33,95 \% \mathrm{Cl} 0.07$ to 1.52 , no evidence of a significant difference between groups

RR $0.75,95 \% \mathrm{Cl} 0.35$ to 1.60 , no evidence of a significant difference between groups 
Table 16. (4.) Results by individual review - immersion in water (Continued)

Adverse effects for women (perineal trauma - sec- 2 (179) ond-degree tear)

Adverse effects for women (perineal trauma - third 1 (60) or fourth degree tear)

Adverse effects for women (postpartum haemor- $\quad 1$ (120) rhage more than $500 \mathrm{ml}$ )

(120)

Adverse effects for women (maternal temperature) $\quad 1(60)$
RR $1.21,95 \% \mathrm{Cl} 0.65$ to 2.24 , no evidence of a significant difference between groups

RR $1.54,95 \% \mathrm{Cl} 0.07$ to 36.11 , no evidence of a significant difference between groups

RR $0.14,95 \% \mathrm{Cl} 0.01$ to 2.71 , no evidence of a significant difference between groups

MD $0.20,95 \% \mathrm{Cl}-0.18$ to 0.58 , no evidence of a significant difference in mean temperature between groups

\begin{tabular}{|c|c|c|}
\hline $\begin{array}{l}\text { Adverse effects for infants (presence of meconium } \\
\text { stained liquor) }\end{array}$ & $2(180)$ & $\begin{array}{l}\mathrm{RR} 1.32,95 \% \mathrm{Cl} 0.63 \text { to } 2.80 \text {, no evidence of a } \\
\text { significant difference between groups }\end{array}$ \\
\hline $\begin{array}{l}\text { Adverse effects for infants (umbilical artery } \mathrm{pH} \text { less } \\
\text { than } 7.20 \text { ) }\end{array}$ & $1(116)$ & $\begin{array}{l}\mathrm{RR} 0.89,95 \% \mathrm{Cl} 0.45 \text { to } 1.75 \text {, no evidence of a } \\
\text { significant difference between groups }\end{array}$ \\
\hline $\begin{array}{l}\text { Adverse effects for infants (antibiotics given to } \\
\text { neonate) }\end{array}$ & $1(60)$ & $\begin{array}{l}\text { RR } 1.50,95 \% \mathrm{Cl} 0.17 \text { to } 13.52 \text {, no evidence of a } \\
\text { significant difference between groups }\end{array}$ \\
\hline $\begin{array}{l}\text { Adverse effects for infants (positive neonatal swab } \\
\text { of ear, mouth or umbilicus) }\end{array}$ & $1(154)$ & $\begin{array}{l}\text { RR } 1.89,95 \% \mathrm{Cl} 0.90 \text { to } 3.96 \text {, no evidence of a } \\
\text { significant difference between groups }\end{array}$ \\
\hline $\begin{array}{l}\text { Admission to special care baby unit/neonatal in- } \\
\text { tensive care unit }\end{array}$ & $2(180)$ & $\begin{array}{l}\text { RR } 0.79,95 \% \mathrm{Cl} 0.25 \text { to } 2.49 \text {, no evidence of a } \\
\text { significant difference between groups }\end{array}$ \\
\hline Apgar score less than seven at five minutes & $1(119)$ & $\begin{array}{l}\text { RR } 4.92,95 \% \mathrm{Cl} 0.24 \text { to } 100.31 \text {, no evidence of } \\
\text { a significant difference between groups }\end{array}$ \\
\hline Poor infant outcomes at long-term follow-up & & Outcome not reported \\
\hline Cost & & Outcome not reported \\
\hline
\end{tabular}

4.2. Early Outcome
versus late
immersion
during the
first stage
of labour (1
study, 200
women)

No. of stud- Results
ies (no.
women)

Pain intensity

Satisfaction with pain relief

Sense of control in labour

Satisfaction with childbirth experience

Effect (negative) on mother/baby interaction

Breastfeeding
Outcome not reported

Outcome not reported

Outcome not reported

Outcome not reported

Outcome not reported

Outcome not reported 
Table 16. (4.) Results by individual review - immersion in water (Continued)

\begin{tabular}{lll} 
Assisted vaginal birth & Outcome not reported \\
\hline Caesarean section & Outcome not reported \\
\hline Adverse effects for women & Outcome not reported \\
\hline Adverse effects for infants (Neonatal infection) & $1(200)$ & $\begin{array}{l}\text { RR 3.00, 95\% Cl 0.12 to } 72.77, \text { no evidence of a } \\
\text { significant difference between groups }\end{array}$ \\
\hline $\begin{array}{ll}\text { Admission to special care baby unit/neonatal in- } \\
\text { tensive care unit }\end{array}$ & Not estimable \\
\hline Apgar score less than seven at one minute & $1(200)$ & Not estimable \\
\hline Poor infant outcomes at long-term follow-up & Outcome not reported \\
\hline Cost & Outcome not reported
\end{tabular}

$\mathrm{I}^{2}$ values reported in table only when random effects analysis has been carried out due to substantial heterogeneity indicated by an $\mathrm{I}^{2}$ value greater than $30 \%$

$\mathrm{Cl}$ : confidence interval; MD: mean difference; RR: risk ratio; VAS: visual analogue scale

Table 17. (5.) Results by individual review - aromatherapy

\section{Aromatherapy for pain management in labour, $\mathbf{2}$ studies, $\mathbf{5 3 5}$ women (Smith 2011c)}

\begin{tabular}{|c|c|c|c|}
\hline $\begin{array}{l}\text { 5.1. Aro- } \\
\text { matherapy } \\
\text { versus stan- } \\
\text { dard care (1 } \\
\text { study, } 513 \\
\text { women) }\end{array}$ & Outcome & $\begin{array}{l}\text { No. of stud- } \\
\text { ies (no. } \\
\text { women) }\end{array}$ & Results \\
\hline & $\begin{array}{l}\text { Pain intensity (women asked to rate their } \\
\text { level of pain after receiving aromathera- } \\
\text { py) }\end{array}$ & $1(513)$ & $\begin{array}{l}\text { No data provided for the standard care group, so data } \\
\text { could not be analysed. }\end{array}$ \\
\hline & Satisfaction with pain relief & & Outcome not reported \\
\hline & Sense of control in labour & & Outcome not reported \\
\hline & Satisfaction with childbirth experience & & Outcome not reported \\
\hline & $\begin{array}{l}\text { Effect (negative) on mother/baby interac- } \\
\text { tion }\end{array}$ & & Outcome not reported \\
\hline & Breastfeeding & & Outcome not reported \\
\hline & Assisted vaginal birth & $1(513)$ & $\begin{array}{l}\text { RR } 1.04,95 \% \mathrm{Cl} 0.48 \text { to } 2.28 \text {, no evidence of a significant } \\
\text { difference }\end{array}$ \\
\hline & Caesarean section & $1(513)$ & $\begin{array}{l}\text { RR } 0.98,95 \% \mathrm{Cl} 0.49 \text { to } 1.94 \text {, no evidence of a significant } \\
\text { difference }\end{array}$ \\
\hline
\end{tabular}


Table 17. (5.) Results by individual review - aromatherapy (Continued)

Adverse effects

Admission to special care baby unit/ neonatal intensive care unit

Apgar score less than seven at five minutes

Poor infant outcomes at long-term follow-up

\begin{tabular}{ll}
\hline & Cost \\
\hline $\begin{array}{l}\text { 5.2. Aro- } \\
\text { mathera- }\end{array}$ & Outcome \\
py (ginger) \\
versus aro- \\
mathera- \\
py (lemon \\
grass) (1 \\
study, 22 \\
women) \\
\hline
\end{tabular}

Pain intensity (assessed on a visual analogue scale before, during or after the bath and 24 hours post-partum (McGill Pain Questionnaire)

\begin{tabular}{|c|c|c|}
\hline \multicolumn{2}{|l|}{ Satisfaction with pain relief } & Outcome not reported \\
\hline \multicolumn{2}{|l|}{ Sense of control in labour } & Outcome not reported \\
\hline \multicolumn{2}{|l|}{ Satisfaction with childbirth experience } & Outcome not reported \\
\hline \multicolumn{2}{|l|}{$\begin{array}{l}\text { Effect (negative) on mother/baby interac- } \\
\text { tion }\end{array}$} & Outcome not reported \\
\hline \multicolumn{2}{|l|}{ Breastfeeding } & Outcome not reported \\
\hline Assisted vaginal birth & $1(22)$ & $\begin{array}{l}\text { RR } 0.83,95 \% \mathrm{Cl} 0.06 \text { to } 11.70 \text {, no evidence of a signifi- } \\
\text { cant difference }\end{array}$ \\
\hline Caesarean section & $1(22)$ & $\begin{array}{l}\text { RR } 2.54,95 \% \mathrm{Cl} 0.11 \text { to } 56.25 \text {, no evidence of a signifi- } \\
\text { cant difference }\end{array}$ \\
\hline $\begin{array}{l}\text { Adverse effects for women (postpartum } \\
\text { haemorrhage) }\end{array}$ & $1(22)$ & $\begin{array}{l}\text { No women in either group had a postpartum haemor- } \\
\text { rhage }\end{array}$ \\
\hline $\begin{array}{l}\text { Admission to special care baby unit/ } \\
\text { neonatal intensive care unit }\end{array}$ & $1(22)$ & No babies in either group were admitted to NICU \\
\hline $\begin{array}{l}\text { Apgar score less than seven at five min- } \\
\text { utes }\end{array}$ & & Outcome not reported \\
\hline $\begin{array}{l}\text { Poor infant outcomes at long-term fol- } \\
\text { low-up }\end{array}$ & & Outcome not reported \\
\hline
\end{tabular}

$1(513)$

Not reported in any trial

RR $0.08,95 \% \mathrm{Cl} 0.00$ to 1.42 , no evidence of a significant difference

Outcome not reported

Outcome not reported

Outcome not reported

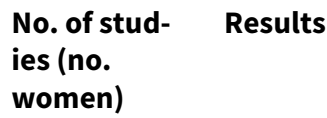

No. of stud- Results

ies (no.

women)

$1(22)$

Median values reported and so data could not be analysed. 
Table 17. (5.) Results by individual review - aromatherapy (Continued)

Cost
Outcome not reported

$\mathrm{I}^{2}$ values reported in table only when random effects analysis has been carried out due to substantial heterogeneity indicated by an $\mathrm{I}^{2}$ value greater than $30 \%$

$\mathrm{Cl}$ : confidence interval; RR: risk ratio

Table 18. (6.) Results by individual review - relaxation techniques

6. Relaxation techniques for pain management in labour, 11 studies, 1574 women (Smith 2011b)

\begin{tabular}{lll}
\hline 6.1. Relaxation (yoga, mu- & Outcome & $\begin{array}{l}\text { No. of stud- } \\
\text { ies (no. }\end{array}$ \\
$\begin{array}{l}\text { sic or audio-analgesia) ver- } \\
\text { sus standard care (11 stud- }\end{array}$ & & women) \\
ies, 1574 women) &
\end{tabular}

\begin{tabular}{|c|c|c|c|}
\hline \multirow[t]{3}{*}{$\begin{array}{l}\text { Relaxation versus standard } \\
\text { care }\end{array}$} & $\begin{array}{l}\text { Pain intensity (Latent phase) (method of } \\
\text { pain assessment not reported) }\end{array}$ & $\begin{array}{l}1 \text { (40 } \\
\text { women) }\end{array}$ & $\begin{array}{l}\mathrm{MD}-1.25,95 \% \mathrm{Cl}-1.97 \text { to }-0.53 \text {, a } \\
\text { significant reduction in pain inten- } \\
\text { sity for women receiving instruc- } \\
\text { tion on relaxation }\end{array}$ \\
\hline & $\begin{array}{l}\text { Pain intensity (Active phase) (method of } \\
\text { pain assessment not reported) }\end{array}$ & $\begin{array}{l}2(74 \\
\text { women) }\end{array}$ & $\begin{array}{l}\mathrm{MD}-2.48,95 \% \mathrm{Cl}-3.13 \text { to }-1.83 \text {, a } \\
\text { significant reduction in pain inten- } \\
\text { sity for women receiving instruc- } \\
\text { tion on relaxation }\end{array}$ \\
\hline & $\begin{array}{l}\text { Pain intensity (memory of pain at } 3 \text { months } \\
\text { follow-up assessed using a Lickert scale } \\
\text { from } 1 \text { to } 7 \text {, where } 7 \text { is "worst imaginable } \\
\text { pain") }\end{array}$ & 1 (904) & $\begin{array}{l}\mathrm{MD} 0.00,95 \% \mathrm{Cl}-0.22 \text { to } 0.22 \text {, no } \\
\text { evidence of a significant difference }\end{array}$ \\
\hline
\end{tabular}

\begin{tabular}{llll}
\hline $\begin{array}{l}\text { Relaxation versus standard } \\
\text { care }\end{array}$ & $\begin{array}{l}\text { Satisfaction with pain relief (postnatal inter- } \\
\text { view 2 hours after delivery) }\end{array}$ & $\begin{array}{l}1 \text { (40 } \\
\text { women) }\end{array}$ & $\begin{array}{l}\text { RR 8.00, 95\% Cl 1.10 to 58.19, a sig- } \\
\text { nificant increase in satisfaction for } \\
\text { women receiving relaxation }\end{array}$ \\
\hline $\begin{array}{l}\text { Relaxation versus standard } \\
\text { care }\end{array}$ & Sense of control in labour & Outcome not reported \\
\hline $\begin{array}{l}\text { Relaxation versus standard } \\
\text { care }\end{array}$ & $\begin{array}{l}\text { Satisfaction with childbirth experience (Wi- } \\
\text { jma Delivery Experience Questionnaire 3 } \\
\text { months postnatal) }\end{array}$ & 1 (904) & $\begin{array}{l}\text { MD - } 0.40,95 \% \mathrm{Cl}-3.47 \text { to } 2.67, \text { no } \\
\text { evidence of a significant difference } \\
\text { (analysis adjusted for clustering) }\end{array}$ \\
\hline $\begin{array}{l}\text { Relaxation versus standard } \\
\text { care }\end{array}$ & Effect on mother/baby interaction & Outcome not reported \\
\hline $\begin{array}{l}\text { Relaxation versus standard } \\
\text { care }\end{array}$ & Breastfeeding & $\begin{array}{l}\text { O (86 } \\
\text { women) }\end{array}$ & $\begin{array}{l}\text { RR 0.07, 95\% Cl 0.01 to 0.50, signif- } \\
\text { icantly fewer women had assist- } \\
\text { ed vaginal birth in the relaxation } \\
\text { group }\end{array}$ \\
\hline $\begin{array}{l}\text { Relaxation versus standard } \\
\text { care }\end{array}$ & Assisted vaginal birth & Outcome not reported \\
\hline \hline
\end{tabular}


Table 18. (6.) Results by individual review - relaxation techniques (Continued)
Relaxation versus standard
Caesarean section
care

\section{$3(990$ women)}

Data from trials not combined in a meta-analysis (2 parallel trials and 1 cluster trial) due to considerable heterogeneity

Outcome not reported

Outcome not reported

Admission to special care baby unit/neonatal intensive care unit

Relaxation versus standard care

\begin{tabular}{llll}
$\begin{array}{l}\text { Relaxation versus standard } \\
\text { care }\end{array}$ & Apgar score less than seven at five minutes & $\begin{array}{l}1 \text { (34 } \\
\text { women) }\end{array}$ & $\begin{array}{l}\text { RR 0.47, 95\% Cl 0.02 to } 10.69, \text { no } \\
\text { evidence of a significant difference }\end{array}$ \\
\hline $\begin{array}{l}\text { Relaxation versus standard } \\
\text { care }\end{array}$ & $\begin{array}{l}\text { Poor infant outcomes at long-term fol- } \\
\text { low-up }\end{array}$ & Outcome not reported \\
\hline
\end{tabular}

RR $0.47,95 \% \mathrm{Cl} 0.02$ to 10.69 , no evidence of a significant difference

\begin{tabular}{lll}
\hline $\begin{array}{l}\text { Relaxation versus standard } \\
\text { care }\end{array}$ & Cost & Outcome not reported
\end{tabular}
care

\begin{tabular}{ll}
\hline Yoga versus standard care & $\begin{array}{l}\text { Pain intensity (Latent phase) (visual ana- } \\
\text { logue sensation of pain scale VASPS) }\end{array} \quad 1(66)$
\end{tabular}

(66) MD $-6.12,95 \% \mathrm{Cl}-11.77$ to -0.47 , a significant reduction in pain intensity for women receiving yoga

\begin{tabular}{ll}
\hline Yoga versus standard care & $\begin{array}{l}\text { Satisfaction with pain relief (method of as- } \\
\text { sessment not reported) }\end{array}$
\end{tabular}

(66)

MD $7.88,95 \% \mathrm{Cl} 1.51$ to 14.25 , significantly greater satisfaction for women receiving yoga

\begin{tabular}{|c|c|c|c|}
\hline Yoga versus standard care & Sense of control in labour & & Outcome not reported \\
\hline Yoga versus standard care & $\begin{array}{l}\text { Satisfaction with childbirth experience (not } \\
\text { clear which tool used for assessment - sev- } \\
\text { eral were reported) }\end{array}$ & $1(66)$ & $\begin{array}{l}\text { MD } 6.34,95 \% \text { Cl } 0.26 \text { to } 12.42 \text {, sig- } \\
\text { nificantly greater satisfaction for } \\
\text { women receiving yoga }\end{array}$ \\
\hline Yoga versus standard care & Effect on mother/baby interaction & & Outcome not reported \\
\hline Yoga versus standard care & Breastfeeding & & Outcome not reported \\
\hline Yoga versus standard care & Assisted vaginal birth & & Outcome not reported \\
\hline Yoga versus standard care & Caesarean section & & Outcome not reported \\
\hline Yoga versus standard care & Adverse effects (for women and infants) & & Outcome not reported \\
\hline Yoga versus standard care & $\begin{array}{l}\text { Admission to special care baby unit/neona- } \\
\text { tal intensive care unit }\end{array}$ & & Outcome not reported \\
\hline Yoga versus standard care & Apgar score less than seven at five minutes & $1(66)$ & $\begin{array}{l}\text { No babies in yoga or control group } \\
\text { had an Apgar score less than seven } \\
\text { at five minutes }\end{array}$ \\
\hline Yoga versus standard care & $\begin{array}{l}\text { Poor infant outcomes at long-term fol- } \\
\text { low-up }\end{array}$ & & Outcome not reported \\
\hline Yoga versus standard care & Cost & & Outcome not reported \\
\hline
\end{tabular}


Table 18. (6.) Results by individual review - relaxation techniques (Continued)

\begin{tabular}{|c|c|c|c|}
\hline \multirow[t]{2}{*}{ Music versus standard care } & $\begin{array}{l}\text { Pain intensity (Latent phase) (visual ana- } \\
\text { logue sensation of pain scale VASPS) }\end{array}$ & $1(60)$ & $\begin{array}{l}\mathrm{MD}-0.17,95 \% \mathrm{Cl}-1.41 \text { to } 1.07 \text {, no } \\
\text { evidence of a significant difference }\end{array}$ \\
\hline & $\begin{array}{l}\text { Pain intensity (Active phase) (visual ana- } \\
\text { logue sensation of pain scale VASPS) }\end{array}$ & $1(60)$ & $\begin{array}{l}\mathrm{MD}-0.18,95 \% \mathrm{Cl}-0.70 \text { to } 0.34 \text {, no } \\
\text { evidence of a significant difference }\end{array}$ \\
\hline Music versus standard care & Satisfaction with pain relief & & Outcome not reported \\
\hline Music versus standard care & Sense of control in labour & & Outcome not reported \\
\hline Music versus standard care & Satisfaction with childbirth experience & & Outcome not reported \\
\hline Music versus standard care & Effect on mother/baby interaction & & Outcome not reported \\
\hline Music versus standard care & Breastfeeding & & Outcome not reported \\
\hline Music versus standard care & Assisted vaginal birth & & Outcome not reported \\
\hline Music versus standard care & Caesarean section & $\begin{array}{l}1 \text { ( } 60 \\
\text { women) }\end{array}$ & $\begin{array}{l}\mathrm{RR} 1.25,95 \% \mathrm{Cl} 0.37 \text { to } 4.21 \text {, no evi- } \\
\text { dence of a significant difference }\end{array}$ \\
\hline Music versus standard care & Adverse effects (for women and infants) & & Outcome not reported \\
\hline Music versus standard care & $\begin{array}{l}\text { Admission to special care baby unit/neona- } \\
\text { tal intensive care unit }\end{array}$ & & Outcome not reported \\
\hline Music versus standard care & Apgar score less than seven at five minutes & & Outcome not reported \\
\hline Music versus standard care & $\begin{array}{l}\text { Poor infant outcomes at long-term fol- } \\
\text { low-up }\end{array}$ & & Outcome not reported \\
\hline Music versus standard care & Cost & & Outcome not reported \\
\hline $\begin{array}{l}\text { Audio-analgesia versus stan- } \\
\text { dard care }\end{array}$ & Pain intensity & & Outcome not reported \\
\hline
\end{tabular}

\begin{tabular}{|c|c|c|c|}
\hline $\begin{array}{l}\text { Audio-analgesia versus stan- } \\
\text { dard care }\end{array}$ & $\begin{array}{l}\text { Satisfaction with pain relief (women's satis- } \\
\text { faction with sea noise) }\end{array}$ & $1(24)$ & $\begin{array}{l}\text { RR } 2.00,95 \% \mathrm{Cl} 0.82 \text { to } 4.89 \text {, no evi- } \\
\text { dence of a significant difference }\end{array}$ \\
\hline $\begin{array}{l}\text { Audio-analgesia versus stan- } \\
\text { dard care }\end{array}$ & Sense of control in labour & & Outcome not reported \\
\hline $\begin{array}{l}\text { Audio-analgesia versus stan- } \\
\text { dard care }\end{array}$ & Satisfaction with childbirth experience & & Outcome not reported \\
\hline $\begin{array}{l}\text { Audio-analgesia versus stan- } \\
\text { dard care }\end{array}$ & Effect on mother/baby interaction & & Outcome not reported \\
\hline $\begin{array}{l}\text { Audio-analgesia versus stan- } \\
\text { dard care }\end{array}$ & Breastfeeding & & Outcome not reported \\
\hline $\begin{array}{l}\text { Audio-analgesia versus stan- } \\
\text { dard care }\end{array}$ & Assisted vaginal birth & & Outcome not reported \\
\hline $\begin{array}{l}\text { Audio-analgesia versus stan- } \\
\text { dard care }\end{array}$ & Caesarean section & & Outcome not reported \\
\hline
\end{tabular}


Table 18. (6.) Results by individual review - relaxation techniques (Continued)

\begin{tabular}{lll}
$\begin{array}{l}\text { Audio-analgesia versus stan- } \\
\text { dard care }\end{array}$ & Adverse effects (for women and infants) & Outcome not reported \\
\hline $\begin{array}{l}\text { Audio-analgesia versus stan- } \\
\text { dard care }\end{array}$ & $\begin{array}{l}\text { Admission to special care baby unit/neona- } \\
\text { tal intensive care unit }\end{array}$ & Outcome not reported \\
\hline $\begin{array}{l}\text { Audio-analgesia versus stan- } \\
\text { dard care }\end{array}$ & Apgar score less than seven at five minutes & Outcome not reported \\
\hline $\begin{array}{l}\text { Audio-analgesia versus stan- } \\
\text { dard care }\end{array}$ & $\begin{array}{l}\text { Poor infant outcomes at long-term fol- } \\
\text { low-up }\end{array}$ & Outcome not reported \\
\hline $\begin{array}{l}\text { Audio-analgesia versus stan- } \\
\text { dard care }\end{array}$ & Cost & Outcome not reported \\
\hline
\end{tabular}

12 values reported in table only when random effects analysis has been carried out due to substantial heterogeneity indicated by an 12 value greater than $30 \%$

$\mathrm{Cl}$ : confidence interval; MD: mean difference; RR: risk ratio

Table 19. (7.) Results by individual review - acupuncture

\section{Acupuncture or acupressure for pain management in labour, 13 studies, 2391 women (Smith 2011a)}

\subsection{Acupuncture or acupressure versus placebo/standard care/no treatment (12 studies, 1858 women)}

Acupuncture versus placebo control

\section{Outcome}

(2)

\author{
No. of stud- Results \\ ies (no. \\ women)
}

Pain intensity $2(240)$
Standardised mean difference (SMD) $0.04,95 \%$ confidence interval $(\mathrm{Cl})-0.22$ to 0.30 , no evidence of a significant difference

Acupuncture versus placebo control Satisfaction with pain relief $\quad 1(150)$

RR $2.38,95 \% \mathrm{Cl} 1.78$ to 3.19 , significantly more women in the acupuncture group were more satisfied with pain relief

\begin{tabular}{|c|c|c|c|}
\hline Acupuncture versus placebo control & Sense of control in labour & & Outcome not reported \\
\hline Acupuncture versus placebo control & $\begin{array}{l}\text { Satisfaction with childbirth expe- } \\
\text { rience }\end{array}$ & & Outcome not reported \\
\hline Acupuncture versus placebo control & $\begin{array}{l}\text { Effect (negative) on mother/baby } \\
\text { interaction }\end{array}$ & & Outcome not reported \\
\hline Acupuncture versus placebo control & Breastfeeding & & Outcome not reported \\
\hline Acupuncture versus placebo control & Assisted vaginal birth & $1(208)$ & $\begin{array}{l}\mathrm{RR} 0.64,95 \% \mathrm{Cl} 0.27 \text { to } 1.50 \text {, no } \\
\text { evidence of a significant differ- } \\
\text { ence }\end{array}$ \\
\hline Acupuncture versus placebo control & Caesarean section & $3(448)$ & $\begin{array}{l}\text { RR } 1.39,95 \% \mathrm{Cl} 0.62 \text { to } 3.10 \text {, no } \\
\text { evidence of a significant differ- } \\
\text { ence }\end{array}$ \\
\hline
\end{tabular}


Table 19. (7.) Results by individual review - acupuncture (Continued)

\begin{tabular}{|c|c|c|c|}
\hline Acupuncture versus placebo control & Adverse effects & & Outcome not reported \\
\hline Acupuncture versus placebo control & $\begin{array}{l}\text { Admission to special care baby } \\
\text { unit/neonatal intensive care unit }\end{array}$ & & Outcome not reported \\
\hline Acupuncture versus placebo control & $\begin{array}{l}\text { Apgar score less than seven at } \\
\text { five minutes }\end{array}$ & $1(208)$ & $\begin{array}{l}\text { RR } 0.32,95 \% \mathrm{Cl} 0.01 \text { to } 7.79 \text {, no } \\
\text { evidence of a significant differ- } \\
\text { ence }\end{array}$ \\
\hline Acupuncture versus placebo control & $\begin{array}{l}\text { Poor infant outcomes at long- } \\
\text { term follow-up }\end{array}$ & & Outcome not reported \\
\hline Acupuncture versus placebo control & Cost & & Outcome not reported \\
\hline Acupuncture versus standard care & Pain intensity & $1(90)$ & $\begin{array}{l}\text { SMD }-0.14,95 \% \mathrm{Cl}-0.55 \text { to } 0.28 \text {, } \\
\text { no evidence of a significant dif- } \\
\text { ference }\end{array}$ \\
\hline Acupuncture versus standard care & Satisfaction with pain relief & $1(90)$ & $\begin{array}{l}\text { RR } 1.08,95 \% \mathrm{Cl} 0.95 \text { to } 1.22 \text {, no } \\
\text { evidence of a significant differ- } \\
\text { ence }\end{array}$ \\
\hline
\end{tabular}

\begin{tabular}{lll}
\hline Acupuncture versus standard care & Sense of control in labour & Outcome not reported \\
\hline Acupuncture versus standard care & $\begin{array}{l}\text { Satisfaction with childbirth expe- } \\
\text { rience }\end{array}$ & Outcome not reported \\
\hline Acupuncture versus standard care & $\begin{array}{l}\text { Effect (negative) on mother/baby } \\
\text { interaction }\end{array}$ & Outcome not reported \\
\hline Acupuncture versus standard care & Breastfeeding & Outcome not reported \\
\hline
\end{tabular}

\begin{tabular}{llll}
\hline Acupuncture versus standard care & Assisted vaginal birth & $3(704)$ & RR 0.67, $95 \%$ Cl 0.46 to 0.98, sig-
\end{tabular}
nificantly fewer women in the acupuncture group had assisted vaginal birth

\begin{tabular}{|c|c|c|c|}
\hline Acupuncture versus standard care & Caesarean section & $2(506)$ & $\begin{array}{l}\text { RR } 0.86,95 \% \mathrm{Cl} 0.47 \text { to } 1.60 \text {, no } \\
\text { evidence of a significant differ- } \\
\text { ence }\end{array}$ \\
\hline Acupuncture versus standard care & Adverse effects & & Outcome not reported \\
\hline Acupuncture versus standard care & $\begin{array}{l}\text { Admission to special care baby } \\
\text { unit/neonatal intensive care unit }\end{array}$ & & Outcome not reported \\
\hline Acupuncture versus standard care & $\begin{array}{l}\text { Apgar score less than seven at } \\
\text { five minutes }\end{array}$ & $3(706)$ & $\begin{array}{l}\mathrm{RR} 0.60,95 \% \mathrm{Cl} 0.12 \text { to } 2.99 \text {, no } \\
\text { evidence of a significant differ- } \\
\text { ence }\end{array}$ \\
\hline Acupuncture versus standard care & $\begin{array}{l}\text { Poor infant outcomes at long- } \\
\text { term follow-up }\end{array}$ & & Outcome not reported \\
\hline Acupuncture versus standard care & Cost & & Outcome not reported \\
\hline Acupuncture versus no treatment & Pain intensity & $1(163)$ & $\begin{array}{l}\text { SMD }-1.00,95 \% \mathrm{Cl}-1.33 \text { to }-0.67 \text {, } \\
\text { significantly more women in the }\end{array}$ \\
\hline
\end{tabular}


Table 19. (7.) Results by individual review - acupuncture (Continued)

acupuncture group reported less intense pain

\begin{tabular}{|c|c|c|c|}
\hline Acupuncture versus no treatment & Satisfaction with pain relief & & Outcome not reported \\
\hline Acupuncture versus no treatment & Sense of control in labour & & Outcome not reported \\
\hline Acupuncture versus no treatment & $\begin{array}{l}\text { Satisfaction with childbirth expe- } \\
\text { rience }\end{array}$ & & Outcome not reported \\
\hline Acupuncture versus no treatment & $\begin{array}{l}\text { Effect (negative) on mother/baby } \\
\text { interaction }\end{array}$ & & Outcome not reported \\
\hline Acupuncture versus no treatment & Breastfeeding & & Outcome not reported \\
\hline Acupuncture versus no treatment & Assisted vaginal birth & $1(163)$ & $\begin{array}{l}\text { RR } 0.49,95 \% \mathrm{Cl} 0.18 \text { to } 1.38 \text {, no } \\
\text { evidence of a significant differ- } \\
\text { ence }\end{array}$ \\
\hline Acupuncture versus no treatment & Caesarean section & $1(163)$ & $\begin{array}{l}\mathrm{RR} 0.76,95 \% \mathrm{Cl} 0.35 \text { to } 1.63 \text {, no } \\
\text { evidence of a significant differ- } \\
\text { ence }\end{array}$ \\
\hline
\end{tabular}

$\begin{array}{lll}\text { Acupuncture versus no treatment } \quad \text { Adverse effects } & \text { Outcome not reported }\end{array}$

Acupuncture versus no treatment

Admission to special care baby unit/neonatal intensive care unit

Outcome not reported

Apgar score less than seven at five minutes

Outcome not reported

Acupuncture versus no treatment

Poor infant outcomes at longterm follow-up

Outcome not reported

Acupuncture versus no treatment

Cost

Outcome not reported

Acupuncture versus no treatment

Pain intensity

$1(120)$

SMD $-0.55,95 \% \mathrm{Cl}-0.92$ to -0.19 significantly more women in the acupressure group reported less intense pain

\begin{tabular}{lll}
\hline Acupressure versus placebo control & Satisfaction with pain relief & Outcome not reported \\
\hline Acupressure versus placebo control & Sense of control in labour & Outcome not reported \\
\hline Acupressure versus placebo control & $\begin{array}{l}\text { Satisfaction with childbirth expe- } \\
\text { rience }\end{array}$ & Outcome not reported \\
\hline
\end{tabular}

Acupressure versus placebo control Effect (negative) on mother/baby interaction

Outcome not reported

\begin{tabular}{|c|c|c|c|}
\hline Acupressure versus placebo control & Breastfeeding & & Outcome not reported \\
\hline Acupressure versus placebo control & Assisted vaginal birth & & Outcome not reported \\
\hline Acupressure versus placebo control & Caesarean section & $1(120)$ & $\begin{array}{l}\text { RR } 0.24,95 \% \mathrm{Cl} 0.11 \text { to } 0.54 \text {, sig- } \\
\text { nificantly fewer women in the }\end{array}$ \\
\hline
\end{tabular}


Table 19. (7.) Results by individual review - acupuncture (Continued)

acupressure group had caesarean section

\begin{tabular}{lll}
\hline Acupressure versus placebo control & Adverse effects & Outcome not reported \\
\hline Acupressure versus placebo control & $\begin{array}{l}\text { Admission to special care baby } \\
\text { unit/neonatal intensive care }\end{array}$ & Outcome not reported \\
\hline Acupressure versus placebo control & $\begin{array}{l}\text { Apgar score less than seven at } \\
\text { five minutes }\end{array}$ & Outcome not reported \\
\hline Acupressure versus placebo control & $\begin{array}{l}\text { Poor infant outcomes at long- } \\
\text { term follow-up }\end{array}$ & Outcome not reported \\
\hline Acupressure versus placebo control & Cost & Outcome not reported \\
\hline Acupressure versus combined control & Pain intensity & SMD -0.42, $95 \%$ Cl -0.65 to -0.18, \\
& & $\begin{array}{l}\text { significantly more women in the } \\
\text { acupressure group reported less } \\
\text { intense pain }\end{array}$
\end{tabular}

\begin{tabular}{|c|c|c|c|}
\hline Acupressure versus combined control & Satisfaction with pain relief & & Outcome not reported \\
\hline Acupressure versus combined control & Sense of control in labour & & Outcome not reported \\
\hline Acupressure versus combined control & $\begin{array}{l}\text { Satisfaction with childbirth expe- } \\
\text { rience }\end{array}$ & $1(211)$ & $\begin{array}{l}\text { MD } 4.80,95 \% \mathrm{Cl}-2.29 \text { to } 11.89 \text {, } \\
\text { no evidence of a significant dif- } \\
\text { ference }\end{array}$ \\
\hline Acupressure versus combined control & $\begin{array}{l}\text { Effect (negative) on mother/baby } \\
\text { interaction }\end{array}$ & & Outcome not reported \\
\hline Acupressure versus combined control & Breastfeeding & & Outcome not reported \\
\hline Acupressure versus combined control & Assisted vaginal birth & $1(222)$ & $\begin{array}{l}\mathrm{RR} 0.81,95 \% \mathrm{Cl} 0.39 \text { to } 1.67 \text {, no } \\
\text { evidence of a significant differ- } \\
\text { ence }\end{array}$ \\
\hline Acupressure versus combined control & Caesarean section & $1(212)$ & $\begin{array}{l}\mathrm{RR} 0.48,95 \% \mathrm{Cl} 0.22 \text { to } 1.04 \text {, no } \\
\text { evidence of a significant differ- } \\
\text { ence }\end{array}$ \\
\hline Acupressure versus combined control & Adverse effects & & Outcome not reported \\
\hline Acupressure versus combined control & $\begin{array}{l}\text { Admission to special care baby } \\
\text { unit/neonatal intensive care }\end{array}$ & & Outcome not reported \\
\hline Acupressure versus combined control & $\begin{array}{l}\text { Apgar score less than seven at } \\
\text { five minutes }\end{array}$ & $1(120)$ & $\begin{array}{l}\text { No infant in either group had an } \\
\text { Apgar score less than seven at } \\
\text { five minutes }\end{array}$ \\
\hline Acupressure versus combined control & $\begin{array}{l}\text { Poor infant outcomes at long- } \\
\text { term follow-up }\end{array}$ & & Outcome not reported \\
\hline Acupressure versus combined control & Cost & & Outcome not reported \\
\hline
\end{tabular}

\subsection{Acupuncture versus a different type of acupuncture (no studies)}


Table 19. (7.) Results by individual review - acupuncture (Continued)

$\begin{array}{lll}\begin{array}{l}\text { 7.3.Acupuncture versus sterile water in- } \\ \text { jection (1study, } 128 \text { women) }\end{array} & \begin{array}{l}\text { No. of stud- } \\ \text { ies (no. } \\ \text { women) }\end{array}\end{array}$

\begin{tabular}{|c|c|c|c|}
\hline Acupuncture versus sterile water injection & Pain intensity & & Outcome not reported \\
\hline Acupuncture versus sterile water injection & Satisfaction with pain relief & $1(128)$ & $\begin{array}{l}\text { MD } 18.60,95 \% \mathrm{Cl} 11.54 \text { to } 25.66 \text {, } \\
\text { significantly more women in the } \\
\text { sterile water group were more } \\
\text { satisfied with pain relief }\end{array}$ \\
\hline
\end{tabular}

\begin{tabular}{lll}
\hline Acupuncture versus sterile water injection & Sense of control in labour & Outcome not reported \\
\hline Acupuncture versus sterile water injection & $\begin{array}{l}\text { Satisfaction with childbirth expe- } \\
\text { rience }\end{array}$ & Outcome not reported \\
\hline Acupuncture versus sterile water injection & $\begin{array}{l}\text { Effect (negative) on mother/baby } \\
\text { interaction }\end{array}$ & Outcome not reported \\
\hline
\end{tabular}

\begin{tabular}{llll}
\hline Acupuncture versus sterile water injection & Breastfeeding & Outcome not reported \\
\hline Acupuncture versus sterile water injection & Assisted vaginal birth & $1(128)$ & $\begin{array}{l}\text { RR 1.60, 95\% Cl 0.47 to 5.39, no } \\
\text { evidence of a significant differ- } \\
\text { ence }\end{array}$ \\
\hline Acupuncture versus sterile water injection & Caesarean section & $1(128)$ & $\begin{array}{l}\text { RR } 1.33,95 \% \mathrm{Cl} 0.37 \text { to } 4.73, \text { no } \\
\text { evidence of a significant differ- } \\
\text { ence }\end{array}$ \\
\hline
\end{tabular}

\begin{tabular}{lll}
\hline Acupuncture versus sterile water injection & Adverse effects & Outcome not reported \\
\hline Acupuncture versus sterile water injection & $\begin{array}{l}\text { Admission to special care baby } \\
\text { unit/neonatal intensive care unit }\end{array}$ & Outcome not reported \\
\hline Acupuncture versus sterile water injection & $\begin{array}{l}\text { Apgar score less than seven at } \\
\text { five minutes }\end{array}$ & Outcome not reported \\
\hline Acupuncture versus sterile water injection & $\begin{array}{l}\text { Poor infant outcomes at long- } \\
\text { term follow-up }\end{array}$ & Outcome not reported \\
\hline Acupuncture versus sterile water injection & Cost & Outcome not reported
\end{tabular}

Combined control $=$ placebo and no treatment

$1^{2}$ values reported in table only when random effects analysis has been carried out due to substantial heterogeneity indicated by an $1^{2}$ value greater than $30 \%$

$\mathrm{Cl}$ : confidence interval; MD: mean difference; RR: risk ratio; SMD: standardised mean difference

Table 20. (8.) Results by individual review - massage, reflexology and other manual methods

8. Massage, reflexology and other manual methods for pain management in labour, 6 studies, 401 women (Smith 2012)

\begin{tabular}{lll}
\hline $\begin{array}{l}\text { 8.1. Mas- } \\
\text { sage ver- }\end{array}$ & Outcome & $\begin{array}{l}\text { No. of stud- } \\
\text { ies (no. } \\
\text { sus stan- }\end{array}$ \\
dard care $(4$ & & women)
\end{tabular}

Pain management for women in labour: an overview of systematic reviews (Review) 
Table 20. (8.) Results by individual review - massage, reflexology and other manual methods (Continued) studies, 239

women)

\begin{tabular}{|c|c|c|}
\hline Pain intensity during first stage of labour & $4(225)$ & $\begin{array}{l}\text { SMD }-0.82,95 \% \mathrm{Cl}-1.17 \text { to }-0.47 \text {, intensity of } \\
\text { pain during the first stage of labour was re- } \\
\text { duced in the massage group compared with } \\
\text { usual care }\end{array}$ \\
\hline Pain intensity during second stage of labour & $2(124)$ & $\begin{array}{l}\text { SMD }-0.98,95 \% \mathrm{Cl}-2.23 \text { to } 0.26 \text {, no differ- } \\
\text { ence between groups in pain intensity }\end{array}$ \\
\hline Pain intensity during third stage of labour & $2(122)$ & $\begin{array}{l}\text { SMD }-1.03,95 \% \mathrm{Cl}-2.17 \text { to } 0.11 \text {, no differ- } \\
\text { ence between groups in pain intensity }\end{array}$ \\
\hline Satisfaction with pain relief & $2(110)$ & $\begin{array}{l}\text { Results not combined for } 2 \text { studies due to } \\
\text { heterogeneity }\end{array}$ \\
\hline Sense of control in labour & $1(40)$ & $\begin{array}{l}\text { MD }-6.10,95 \% \mathrm{Cl}-13.11 \text { to } 0.91 \text {, no differ- } \\
\text { ence between groups in sense of control in } \\
\text { labour }\end{array}$ \\
\hline Satisfaction with childbirth experience & & Outcome not reported \\
\hline Effect on mother/baby interaction & & Outcome not reported \\
\hline Breastfeeding & & Outcome not reported \\
\hline Assisted vaginal birth & $2(105)$ & $\begin{array}{l}\text { RR } 0.46,95 \% \mathrm{Cl} 0.14 \text { to } 1.50 \text {, no evidence of a } \\
\text { significant difference between groups }\end{array}$ \\
\hline Caesarean section & $2(105)$ & $\begin{array}{l}\text { RR } 0.73,95 \% \mathrm{Cl} 0.24 \text { to } 2.22 \text {, no evidence of a } \\
\text { significant difference between groups }\end{array}$ \\
\hline Adverse effects for women and infants & & Outcome not reported \\
\hline $\begin{array}{l}\text { Admission to special care baby unit/neonatal inten- } \\
\text { sive care unit }\end{array}$ & $1(44)$ & $\begin{array}{l}\mathrm{RR} 1.93,95 \% \mathrm{Cl} 0.13 \text { to } 28.79 \text {, no evidence of } \\
\text { a significant difference between groups }\end{array}$ \\
\hline Apgar score less than seven at five minutes & & Outcome not reported \\
\hline Poor infant outcomes at long-term follow-up & & Outcome not reported \\
\hline Cost & & Outcome not reported \\
\hline
\end{tabular}

\title{
8.2. One \\ manual \\ method ver- \\ sus differ- \\ ent manual \\ method (no \\ studies)
}

8.3. Mas-
sage ver-
sus music (1
study, 101
women)

\author{
No. of stud- Results \\ ies (no. \\ women)
}


Table 20. (8.) Results by individual review - massage, reflexology and other manual methods (Continued)

Pain intensity - number of women reporting severe $\quad 1(101) \quad$ RR $0.40,95 \% \mathrm{Cl} 0.18$ to 0.89 , significantly pain fewer women in the massage group reported severe pain compared to the music group

Satisfaction with pain relief

Outcome not reported

Sense of control in labour

Outcome not reported

Satisfaction with childbirth experience

Outcome not reported

Effect on mother/baby interaction

Outcome not reported

Breastfeeding

Outcome not reported

Assisted vaginal birth

Outcome not reported

Caesarean section

Outcome not reported

Adverse effects for women and infants

Outcome not reported

Admission to special care baby unit/neonatal intensive care unit

Apgar score less than seven at five minutes

Outcome not reported

Poor infant outcomes at long-term follow-up

Outcome not reported

Cost

Outcome not reported

\begin{tabular}{|c|c|c|c|}
\hline $\begin{array}{l}\text { 8.3. Mas- } \\
\text { sage versus } \\
\text { breathing } \\
\text { exercises } \\
\text { (1 study, } 28 \\
\text { women) }\end{array}$ & Outcome & $\begin{array}{l}\text { No. of stud- } \\
\text { ies (no. } \\
\text { women) }\end{array}$ & Results \\
\hline & Pain intensity & & Outcome not reported \\
\hline & Satisfaction with pain relief & & Outcome not reported \\
\hline & Sense of control in labour & & Outcome not reported \\
\hline & Satisfaction with childbirth experience & & Outcome not reported \\
\hline & Effect on mother/baby interaction & & Outcome not reported \\
\hline & Breastfeeding & & Outcome not reported \\
\hline & Assisted vaginal birth & & Outcome not reported \\
\hline & Caesarean section & & Outcome not reported \\
\hline & Adverse effects for women and infants & & Outcome not reported \\
\hline & $\begin{array}{l}\text { Admission to special care baby unit/neonatal inten- } \\
\text { sive care unit }\end{array}$ & & Outcome not reported \\
\hline
\end{tabular}


Table 20. (8.) Results by individual review - massage, reflexology and other manual methods (Continued)

Apgar score less than seven at five minutes

Poor infant outcomes at long-term follow-up

Cost
Outcome not reported

Outcome not reported

Outcome not reported

$\mathrm{I}^{2}$ values reported in table only when random effects analysis has been carried out due to substantial heterogeneity indicated by an $\mathrm{I}^{2}$ value greater than $30 \%$

$\mathrm{Cl}$ : confidence interval; MD: mean difference; RR: risk ratio; SMD: standardised mean difference

Table 21. (9.) Results by individual review - TENS

\section{Transcutaneous electrical nerve stimulation (TENS) for pain management in labour, 17 studies, 1455 women (Dowswell 2009)}

\subsection{TENS versus placebo or standard care (17 studies, 1455 women)}

TENS (to the back) versus placebo/usual care

$\begin{array}{ll}\text { Outcome } & \begin{array}{l}\text { No. of stud- Results } \\ \text { ies (no. } \\ \text { women) }\end{array}\end{array}$

Pain intensity - number of $2(147)$ women reporting severe pain during labour
Average RR $0.67,95 \% \mathrm{Cl} 0.32$ to 1.40 (random effects; heterogeneity: $1^{2}=49 \%$, $\mathrm{Tau}^{2}=0.18$, Chi $^{2}$ test for heterogeneity $\mathrm{P}$ $=0.16)$, no evidence of a significant difference

\begin{tabular}{|c|c|c|c|}
\hline $\begin{array}{l}\text { TENS (to the back) versus place- } \\
\text { bo/usual care }\end{array}$ & Pain intensity - pain scores & $2(299)$ & $\begin{array}{l}\text { Average SMD }-1.01,95 \% \mathrm{Cl}-3.00 \text { to } 0.97 \\
\text { (random effects: heterogeneity: } \mathrm{I}^{2}=93 \% \text {, } \\
\mathrm{Tau}^{2}=1.92, \mathrm{Chi}^{2} \text { test for heterogeneity } \mathrm{P} \\
=0.0002 \text { ), no evidence of a significant dif- } \\
\text { ference }\end{array}$ \\
\hline
\end{tabular}

\begin{tabular}{llll}
\hline $\begin{array}{l}\text { TEN (to the back) versus place- } \\
\text { bo/usual care }\end{array}$ & Satisfaction with pain relief & $5(452)$ & $\begin{array}{l}\text { RR 1.25, 95\% Cl 0.98 to 1.60, no evidence } \\
\text { of a significant difference }\end{array}$ \\
\hline TENS (to the back) versus usual care & Sense of control in labour & $1(24)$ & $\begin{array}{l}\text { Data not analysed (standard deviations } \\
\text { inconsistent) }\end{array}$ \\
\hline TENS (to the back) versus usual care & $\begin{array}{l}\text { Satisfaction with childbirth ex- } \\
\text { perience }\end{array}$ & 1 (24) & $\begin{array}{l}\text { Data not analysed (standard deviations } \\
\text { inconsistent) }\end{array}$ \\
\hline $\begin{array}{l}\text { TENS (to the back) } \\
\text { versus placebo/usual care }\end{array}$ & $\begin{array}{l}\text { Effect (negative) on mother/ba- } \\
\text { by interaction }\end{array}$ & Outcome not reported \\
\hline $\begin{array}{l}\text { TENS (to the back) } \\
\text { versus placebo/usual care }\end{array}$ & Breastfeeding & Outcome not reported \\
\hline $\begin{array}{l}\text { TENS (to the back) versus place- } \\
\text { bo/usual care }\end{array}$ & Assisted vaginal birth & $7(840)$ & $\begin{array}{l}\text { RR 0.82, 95\% Cl 0.56 to 1.19, no evidence } \\
\text { of a significant difference }\end{array}$ \\
\hline $\begin{array}{l}\text { TENS (to the back) versus place- } \\
\text { bo/usual care }\end{array}$ & Caesarean section & $\begin{array}{l}\text { RR 1.35, 95\% Cl 0.84 to 2.17, no evidence } \\
\text { of a significant difference }\end{array}$ \\
\hline
\end{tabular}


Table 21. (9.) Results by individual review - TENS (Continued)

\begin{tabular}{|c|c|c|c|}
\hline TENS (to the back) versus control & $\begin{array}{l}\text { Adverse effects for infants (fetal } \\
\text { distress) }\end{array}$ & $1(200)$ & $\begin{array}{l}\text { RR } 0.33,95 \% \mathrm{Cl} 0.01 \text { to } 8.09 \text {, no evidence } \\
\text { of a significant difference }\end{array}$ \\
\hline $\begin{array}{l}\text { TENS (to the back) } \\
\text { versus placebo/usual care }\end{array}$ & $\begin{array}{l}\text { Admission to special care ba- } \\
\text { by unit/neonatal intensive care } \\
\text { unit }\end{array}$ & & Outcome not reported \\
\hline $\begin{array}{l}\text { TENS (to the back) } \\
\text { versus placebo/usual care }\end{array}$ & $\begin{array}{l}\text { Apgar score less than seven at } \\
\text { five minutes }\end{array}$ & & Outcome not reported \\
\hline $\begin{array}{l}\text { TENS (to the back) } \\
\text { versus placebo/usual care }\end{array}$ & $\begin{array}{l}\text { Poor infant outcomes at long- } \\
\text { term follow-up }\end{array}$ & & Outcome not reported \\
\hline $\begin{array}{l}\text { TENS (to the back) } \\
\text { versus placebo/usual care }\end{array}$ & Cost & & Outcome not reported \\
\hline $\begin{array}{l}\text { TENS (to acupuncture points) versus } \\
\text { placebo }\end{array}$ & $\begin{array}{l}\text { Pain intensity - number of } \\
\text { women reporting severe pain } \\
\text { during labour }\end{array}$ & $2(290)$ & $\begin{array}{l}\text { RR } 0.41,95 \% \mathrm{Cl} 0.31 \text { to } 0.54 \text {, significantly } \\
\text { fewer women in TENS group reported se- } \\
\text { vere pain compared with controls }\end{array}$ \\
\hline $\begin{array}{l}\text { TENS (to acupuncture points) versus } \\
\text { control (no pain relief) }\end{array}$ & Satisfaction with pain relief & $1(90)$ & $\begin{array}{l}\text { RR } 4.10,95 \% \mathrm{CI} 1.81 \text { to } 9.29 \text {, significant- } \\
\text { ly more women in TENS group were satis- } \\
\text { fied with pain relief compared with con- } \\
\text { trols }\end{array}$ \\
\hline $\begin{array}{l}\text { TENS (to acupuncture points) versus } \\
\text { placebo/control (no treatment) }\end{array}$ & Sense of control in labour & & Outcome not reported \\
\hline
\end{tabular}

\begin{tabular}{|c|c|c|c|}
\hline $\begin{array}{l}\text { TENS (to acupuncture points) versus } \\
\text { placebo/control (no treatment) }\end{array}$ & $\begin{array}{l}\text { Satisfaction with childbirth ex- } \\
\text { perience }\end{array}$ & & Outcome not reported \\
\hline $\begin{array}{l}\text { TENS (to acupuncture points) versus } \\
\text { placebo/control (no treatment) }\end{array}$ & $\begin{array}{l}\text { Effect (negative) on mother/ba- } \\
\text { by interaction }\end{array}$ & & Outcome not reported \\
\hline $\begin{array}{l}\text { TENS (to acupuncture points) versus } \\
\text { placebo/control (no treatment) }\end{array}$ & Breastfeeding & & Outcome not reported \\
\hline $\begin{array}{l}\text { TENS (to acupuncture points) versus } \\
\text { placebo }\end{array}$ & Assisted vaginal birth & $1(100)$ & $\begin{array}{l}\mathrm{RR} 4.50,95 \% \mathrm{Cl} 1.02 \text { to } 19.79 \text {, significantly } \\
\text { more women in TENS group had assisted } \\
\text { vaginal births }\end{array}$ \\
\hline $\begin{array}{l}\text { TENS (Limoge current to cranium) } \\
\text { versus placebo }\end{array}$ & Assisted vaginal birth & $1(20)$ & $\begin{array}{l}\mathrm{RR} 0.67,95 \% \mathrm{Cl} 0.14 \text { to } 3.17 \text {, no evidence } \\
\text { of a significant difference }\end{array}$ \\
\hline $\begin{array}{l}\text { TENS (to acupuncture points) versus } \\
\text { placebo }\end{array}$ & Caesarean section & $1(100)$ & $\begin{array}{l}\mathrm{RR} 1.50,95 \% \mathrm{Cl} 0.26 \text { to } 8.60 \text {, no evidence } \\
\text { of a significant difference }\end{array}$ \\
\hline $\begin{array}{l}\text { TENS (Limoge current to cranium) } \\
\text { versus placebo }\end{array}$ & Caesarean section & $1(20)$ & $\begin{array}{l}\text { RR } 1.00,95 \% \mathrm{Cl} 0.07 \text { to } 13.87 \text {, no evidence } \\
\text { of a significant difference }\end{array}$ \\
\hline $\begin{array}{l}\text { TENS (to acupuncture points) versus } \\
\text { placebo }\end{array}$ & $\begin{array}{l}\text { Adverse effects for infants (fetal } \\
\text { distress) }\end{array}$ & $1(100)$ & $\begin{array}{l}\text { RR } 1.00,95 \% \mathrm{Cl} 0.06 \text { to } 15.55) \text {, no evi- } \\
\text { dence of a significant difference }\end{array}$ \\
\hline
\end{tabular}


Table 21. (9.) Results by individual review - TENS (Continued)

\begin{tabular}{lll}
$\begin{array}{l}\text { TENS (to acupuncture points) versus } \\
\text { placebo/control (no treatment) }\end{array}$ & $\begin{array}{l}\text { Admission to special care ba- } \\
\text { by unit/neonatal intensive care } \\
\text { unit }\end{array}$ & Outcome not reported \\
\hline $\begin{array}{l}\text { TENS (to acupuncture points) versus } \\
\text { placebo/control (no treatment) }\end{array}$ & $\begin{array}{l}\text { Apgar score less than seven at } \\
\text { five minutes }\end{array}$ & Outcome not reported \\
\hline $\begin{array}{l}\text { TENS (to acupuncture points) versus } \\
\text { placebo/control (no treatment) }\end{array}$ & $\begin{array}{l}\text { Poor infant outcomes at long- } \\
\text { term follow-up }\end{array}$ & Outcome not reported \\
\hline $\begin{array}{l}\text { TENS (to acupuncture points) versus } \\
\text { placebo/control (no treatment) }\end{array}$ & Cost & Outcome not reported
\end{tabular}

\subsection{TENS versus a different type of TENS (no studies)}

$\begin{array}{lll}\begin{array}{l}\text { 9.3. TENS versus sterile water in- Outcome } \\ \text { jection (1 study, } 22 \text { women) }\end{array} & \begin{array}{l}\text { No. of stud- } \\ \text { ies (no. } \\ \text { women) }\end{array}\end{array}$

Pain intensity - pain score mea- $\quad 1(22)$ sured using visual analogue scale (VAS) in labour

SMD 5.45, 95\% CI 3.49 to 7.42 , women in the TENS group more likely to have a higher mean pain score than women in the sterile water group, "The study measured pain on a scale with scores recorded in millimetres, it was not clear how the $10 \mathrm{~cm}$ scale was labelled and the reported standard deviations are much smaller than would be expected with this type of scale, therefore results should be interpreted with caution".

\begin{tabular}{lll}
\hline & Satisfaction with pain relief & Outcome not reported \\
\hline Sense of control in labour & $1(22)$ & Data not suitable for analysis \\
\hline $\begin{array}{l}\text { Satisfaction with childbirth ex- } \\
\text { perience }\end{array}$ & 1 (22) & Data not suitable for analysis \\
\hline $\begin{array}{l}\text { Effect (negative) on mother/ba- } \\
\text { by interaction }\end{array}$ & Outcome not reported \\
\hline Breastfeeding & Outcome not reported \\
\hline Assisted vaginal birth & Outcome not reported \\
\hline $\begin{array}{l}\text { Caesarean section } \\
\text { Adverse effects }\end{array}$ & $\begin{array}{l}\text { RR 7.62, 95\% Cl 0.46 to 126.40, no evi- } \\
\text { dence of a significant difference }\end{array}$ \\
\hline $\begin{array}{l}\text { Admission to special care ba- } \\
\text { by unit/neonatal intensive care } \\
\text { unit }\end{array}$ & Outcome not reported \\
\hline $\begin{array}{l}\text { Apgar score less than seven at } \\
\text { five minutes }\end{array}$ & \begin{tabular}{l} 
Outcome not reported \\
\hline
\end{tabular}
\end{tabular}


Table 21. (9.) Results by individual review - TENS (Continued)

Poor infant outcomes at long- Outcome not reported term follow-up

\begin{tabular}{ll}
\hline Cost & Outcome not reported \\
\hline
\end{tabular}

$\mathrm{I}^{2}$ values reported in table only when random effects analysis has been carried out due to substantial heterogeneity indicated by an $\mathrm{I}^{2}$ value greater than $30 \%$

$\mathrm{Cl}$ : confidence interval; RR: risk ratio; SMD: standardised mean difference

\section{Table 22. (1.) Results by individual review - inhaled analgesia}

\section{Inhaled analgesia for pain management in labour, 26 studies, 2967 women (Klomp 2012)}

\begin{tabular}{|c|c|c|c|}
\hline \multirow[t]{11}{*}{$\begin{array}{l}\text { 1.1. Inhaled } \\
\text { analge- } \\
\text { sia versus } \\
\text { placebo } \\
\text { control/no } \\
\text { treatment } \\
\text { (9 stud- } \\
\text { ies, } 1495 \\
\text { women) }\end{array}$} & Outcome & $\begin{array}{l}\text { No. of stud- } \\
\text { ies (no. } \\
\text { women) }\end{array}$ & Results \\
\hline & $\begin{array}{l}\text { Pain intensity during the first stage of } \\
\text { labour (severe/extreme pain) }\end{array}$ & $2(310)$ & $\begin{array}{l}\text { Average RR } 0.06,95 \% \mathrm{Cl} 0.01 \text { to } 0.34 \text {, (random effects: } \\
\text { heterogeneity: } \mathrm{I}^{2}=51 \%, \mathrm{Tau}^{2}=1.08, \mathrm{Chi}^{2} \text { test for het- } \\
\text { erogeneity } \mathrm{P}=0.15 \text { ), significantly fewer women in the } \\
\text { inhaled analgesia group experienced severe pain }\end{array}$ \\
\hline & $\begin{array}{l}\text { Pain intensity during the first stage of } \\
\text { labour (VAS 0-10 after one hour) }\end{array}$ & $1(509)$ & $\begin{array}{l}\mathrm{MD}-3.50,95 \% \mathrm{Cl}-3.75 \text { to }-3.25 \text {, mean pain score was } \\
\text { significantly lower in the inhaled analgesia group }\end{array}$ \\
\hline & Satisfaction with pain relief & & Outcome not reported \\
\hline & Sense of control in labour & & Outcome not reported \\
\hline & Satisfaction with childbirth experience & & Outcome not reported \\
\hline & Effect on mother/baby interaction & & Outcome not reported \\
\hline & Breastfeeding & & Outcome not reported \\
\hline & Assisted vaginal birth & $1(200)$ & $\begin{array}{l}\text { RR } 1.50,95 \% \mathrm{Cl} 0.44 \text { to } 5.15 \text {, no evidence of a significant } \\
\text { difference between groups }\end{array}$ \\
\hline & Caesarean section & $3(465)$ & $\begin{array}{l}\text { RR } 1.20,95 \% \mathrm{Cl} 0.75 \text { to } 1.91 \text {, no evidence of a significant } \\
\text { difference between groups }\end{array}$ \\
\hline & Adverse effects for women (vomiting) & $2(619)$ & $\begin{array}{l}\text { RR } 9.05,95 \% \mathrm{Cl} 1.18 \text { to } 69.32 \text {, significantly more women } \\
\text { in the inhaled analgesia group experienced vomiting }\end{array}$ \\
\hline
\end{tabular}


Table 22. (1.) Results by individual review - inhaled analgesia (Continued)

Adverse effects for women (nausea) $\quad 1$ (509) $\quad$ RR 43.10, $95 \% \mathrm{Cl} 2.63$ to 706.74 , significantly more women in the inhaled analgesia group experienced nausea (very wide $\mathrm{Cl}$ )

Adverse effects for women (dizziness) $\quad 1$ (509) $\quad$ RR $113.98,95 \% \mathrm{Cl} 7.09$ to 1833.69 , significantly more women in the inhaled analgesia group experienced dizziness (extremely wide $\mathrm{Cl}$ )

Adverse effects for women (drowsiness) $\quad 1$ (509)

RR $77.59,95 \% \mathrm{Cl} 4.80$ to 1254.96 , significantly more women in the inhaled analgesia group experienced drowsiness (extremely wide $\mathrm{Cl}$ )

\begin{tabular}{|c|c|c|c|}
\hline & $\begin{array}{l}\text { Adverse effects for infants (neonatal as- } \\
\text { phyxia) }\end{array}$ & $1(110)$ & $\begin{array}{l}\text { RR } 1.11,95 \% \mathrm{Cl} 0.26 \text { to } 4.73 \text {, no significant difference } \\
\text { between groups }\end{array}$ \\
\hline & $\begin{array}{l}\text { Admission to special care baby unit/ } \\
\text { neonatal intensive care unit }\end{array}$ & & Outcome not reported \\
\hline & $\begin{array}{l}\text { Apgar score less than seven at five min- } \\
\text { utes }\end{array}$ & $1(200)$ & $\begin{array}{l}\text { RR } 9.00,95 \% \mathrm{Cl} 0.49 \text { to } 165.00 \text {, no significant difference } \\
\text { between groups }\end{array}$ \\
\hline & $\begin{array}{l}\text { Poor infant outcomes at long-term fol- } \\
\text { low-up }\end{array}$ & & Outcome not reported \\
\hline & Cost & & Outcome not reported \\
\hline $\begin{array}{l}\text { 1.2.1. In- } \\
\text { haled anal- } \\
\text { gesia ver- } \\
\text { sus a dif- } \\
\text { ferent type } \\
\text { of inhaled } \\
\text { analgesia, } \\
\text { nitrous ox- } \\
\text { ide versus } \\
\text { flurane (14 } \\
\text { studies, } 752 \\
\text { women) }\end{array}$ & Outcome & $\begin{array}{l}\text { No. of stud- } \\
\text { ies (no. } \\
\text { women) }\end{array}$ & Results \\
\hline
\end{tabular}

Pain intensity - pain score -VAS 0-100 first $3(123) \quad$ Average mean difference MD $13.87,95 \% \mathrm{Cl} 4.02$ to stage of labour - where 100 is most severe 23.72, (random effects: heterogeneity: $\left.\right|^{2}=64 \%$, Tau $^{2}$ pain

$=47.26$, Chi $^{2}$ test for heterogeneity $\mathrm{P}=0.06$ ), the mean pain score was significantly higher in the nitrous oxide group compared to the flurane group (flurane better pain relief) 
Table 22. (1.) Results by individual review - inhaled analgesia (Continued)

Pain intensity - pain relief score - VAS

$0-100$, where 100 is the most pain relief
$2(140)$

Average mean difference MD $-16.92,95 \% \mathrm{Cl}-27.64$ to -6.20 , (random effects: heterogeneity: $\left.\right|^{2}=70 \%$, Tau $^{2}=$ 42.58.80, Chi 2 test for heterogeneity $P=0.07$ ), the mean pain relief score was significantly lower in the nitrous oxide group compared to the flurane group (flurane better pain relief)

\begin{tabular}{|c|c|c|}
\hline $\begin{array}{l}\text { Satisfaction with pain relief during the } \\
\text { first and second stages of labour (propor- } \\
\text { tion with considerable or complete relief) }\end{array}$ & $2(98)$ & $\begin{array}{l}\text { RR } 0.97,95 \% \mathrm{Cl} 0.80 \text { to } 1.18 \text {, no significant difference } \\
\text { between groups }\end{array}$ \\
\hline $\begin{array}{l}\text { Satisfaction with pain relief during the } \\
\text { second stage of labour (proportion with } \\
\text { good or excellent pain relief) }\end{array}$ & $4(323)$ & $\begin{array}{l}\text { RR } 0.89,95 \% \mathrm{Cl} 0.78 \text { to } 1.01 \text {, no significant difference } \\
\text { between groups }\end{array}$ \\
\hline Sense of control in labour & & Outcome not reported \\
\hline Satisfaction with childbirth experience & & Outcome not reported \\
\hline Effect on mother/baby interaction & & Outcome not reported \\
\hline Breastfeeding & & Outcome not reported \\
\hline Assisted vaginal birth & $5(371)$ & $\begin{array}{l}\text { RR } 0.74,95 \% \mathrm{Cl} 0.51 \text { to } 1.05 \text {, there were fewer assisted } \\
\text { vaginal births in the nitrous oxide group, although this } \\
\text { difference did not reach statistical significance }\end{array}$ \\
\hline Caesarean section & $1(98)$ & Not estimable - no CS in either group \\
\hline Adverse effects for women (amnesia) & $4(281)$ & $\begin{array}{l}\text { Average RR } 0.26,95 \% \mathrm{Cl} 0.03 \text { to } 2.38 \text {, (random effects: } \\
\text { heterogeneity: } \mathrm{I}^{2}=74 \%, \mathrm{Tau}^{2}=2.76, \mathrm{Chi}^{2} \text { test for het- } \\
\text { erogeneity } \mathrm{P}=0.02 \text { ), there was less amnesia reported } \\
\text { in the nitrous oxide group, although this difference did } \\
\text { not reach statistical significance }\end{array}$ \\
\hline Adverse effects for women (drowsiness) & $1(80)$ & Not estimable - no drowsiness in either group \\
\hline $\begin{array}{l}\text { Adverse effects for women (drowsiness), } \\
\text { VAS 0-100 mm }\end{array}$ & $2(57)$ & $\begin{array}{l}\mathrm{MD}-12.97,95 \% \mathrm{Cl}-22.33 \text { to }-3.62 \text {, significantly lower } \\
\text { mean drowsiness score with nitrous oxide compared to } \\
\text { flurane group, (nitrous oxide better) }\end{array}$ \\
\hline Adverse effects for women (dizziness) & $2(204)$ & $\begin{array}{l}\text { RR } 0.80,95 \% \mathrm{Cl} 0.40 \text { to } 1.61 \text {, no evidence of a significant } \\
\text { difference between groups }\end{array}$ \\
\hline Adverse effects for women (nausea) & $6(378)$ & $\begin{array}{l}\mathrm{RR} 3.30,95 \% \mathrm{Cl} 1.64 \text { to } 6.63 \text {, significantly more women } \\
\text { in the nitrous oxide group experienced nausea com- } \\
\text { pared to the flurane group }\end{array}$ \\
\hline
\end{tabular}


Table 22. (1.) Results by individual review - inhaled analgesia (Continued)

Adverse effects for women (vomiting) $\quad 4$ (261) $\quad$ RR 2.66, 95\% Cl 1.06 to 6.70 , significantly more women in the nitrous oxide group experienced vomiting compared to the flurane group

Adverse effects for women (blood loss) $\quad 2$ (185) $\quad \mathrm{MD} 6.00,95 \% \mathrm{Cl}-32.91$ to 44.91 , no significant difference between groups in blood loss

Adverse effects for infants (neurologic

$3(170)$ and adaptive capacity score NACS $<35$ at 2 hours after delivery)

Admission to special care baby unit/ neonatal intensive care

Apgar score less than seven at five min- $\quad 6(409)$ utes
RR $1.45,95 \% \mathrm{Cl} 0.91$ to 2.33 , more babies in the nitrous oxide group had a NACS score of $<35$, although this difference did not reach statistical significance

\begin{tabular}{|c|c|c|c|}
\hline & $\begin{array}{l}\text { Admission to special care baby unit/ } \\
\text { neonatal intensive care }\end{array}$ & & Outcome not reported \\
\hline & $\begin{array}{l}\text { Apgar score less than seven at five min- } \\
\text { utes }\end{array}$ & $6(409)$ & $\begin{array}{l}\text { RR } 0.22,95 \% \mathrm{Cl} 0.01 \text { to } 4.47 \text {, no significant difference } \\
\text { between groups (N.B. } 5 \text { of trials had no babies with Ap- } \\
\text { gar score less than seven at five minutes) }\end{array}$ \\
\hline & $\begin{array}{l}\text { Poor infant outcomes at long-term fol- } \\
\text { low-up }\end{array}$ & & Outcome not reported \\
\hline & Cost & & Outcome not reported \\
\hline \multirow{9}{*}{$\begin{array}{l}\text { 1.2.2. In- } \\
\text { haled anal- } \\
\text { gesia of one } \\
\text { strength } \\
\text { versus a } \\
\text { different } \\
\text { strength ( } 2 \\
\text { studies, } 625 \\
\text { women) }\end{array}$} & Outcome & $\begin{array}{l}\text { No. of stud- } \\
\text { ies (no. } \\
\text { women) }\end{array}$ & Results \\
\hline & Pain intensity & & Outcome not reported \\
\hline & $\begin{array}{l}\text { Satisfaction with pain relief during the } \\
\text { first stage of labour (good to complete } \\
\text { pain relief) }\end{array}$ & $1(501)$ & $\begin{array}{l}\text { RR } 1.05,95 \% \mathrm{Cl} 0.94 \text { to } 1.17 \text {, no significant difference } \\
\text { between } 50 \% \text { and } 70 \% \text { nitrous oxide groups }\end{array}$ \\
\hline & $\begin{array}{l}\text { Satisfaction with pain relief during the } \\
\text { second stage of labour (good to complete } \\
\text { relief) }\end{array}$ & $1(501)$ & $\begin{array}{l}\text { RR } 0.97,95 \% \mathrm{Cl} 0.87 \text { to } 1.08 \text {, no significant difference } \\
\text { between } 50 \% \text { and } 70 \% \text { nitrous oxide groups }\end{array}$ \\
\hline & Sense of control in labour & & Outcome not reported \\
\hline & Satisfaction with childbirth experience & & Outcome not reported \\
\hline & Effect on mother/baby interaction & & Outcome not reported \\
\hline & Breastfeeding & & Outcome not reported \\
\hline & Assisted vaginal birth & $1(501)$ & $\begin{array}{l}\text { RR } 0.83,95 \% \mathrm{Cl} 0.61 \text { to } 1.14 \text {, fewer assisted vaginal } \\
\text { births in the } 50 \% \text { nitrous oxide group, although this dif- } \\
\text { ference did not reach statistical significance }\end{array}$ \\
\hline
\end{tabular}


Table 22. (1.) Results by individual review - inhaled analgesia (Continued)

Caesarean section $\quad 1(501) \quad$ RR $0.31,95 \% \mathrm{Cl} 0.06$ to 1.53 , fewer caesarean sections in the $50 \%$ nitrous oxide group, although this difference did not reach statistical significance

Adverse effects for women (vomiting) $\quad 1$ (501)

RR $1.29,95 \% \mathrm{Cl} 0.86$ to 1.94 , less vomiting reported in the $70 \%$ nitrous oxide group, although this difference did not reach statistical significance

Adverse effects for women (postpartum 1 (501)

RR $0.80,95 \% \mathrm{Cl} 0.38$ to 1.70 , fewer women experienced haemorrhage) postpartum haemorrhage in the $50 \%$ nitrous oxide group, although this difference did not reach statistical significance

Adverse effects for women (hypoxaemia)

$1(501)$

RR $1.00,95 \% \mathrm{Cl} 0.07$ to 14.21 , no significant difference between $50 \%$ and $70 \%$ nitrous oxide groups

\begin{tabular}{|c|c|c|c|}
\hline & Adverse effects for infants & & Outcome not reported \\
\hline & $\begin{array}{l}\text { Admission to special care baby unit/ } \\
\text { neonatal intensive care unit }\end{array}$ & & Outcome not reported \\
\hline & $\begin{array}{l}\text { Apgar score less than seven at five min- } \\
\text { utes }\end{array}$ & & Outcome not reported \\
\hline & $\begin{array}{l}\text { Poor infant outcomes at long-term fol- } \\
\text { low-up }\end{array}$ & & Outcome not reported \\
\hline & Cost & & Outcome not reported \\
\hline \multirow{2}{*}{$\begin{array}{l}\text { 1.2.3. In- } \\
\text { haled anal- } \\
\text { gesia using } \\
\text { one type } \\
\text { of delivery } \\
\text { system ver- } \\
\text { sus a differ- } \\
\text { ent system } \\
\text { ( } 2 \text { studies, } \\
75 \text { women) }\end{array}$} & Outcome & $\begin{array}{l}\text { No. of stud- } \\
\text { ies (no. } \\
\text { women) }\end{array}$ & Results \\
\hline & Pain intensity & & Outcome not reported \\
\hline \multirow{5}{*}{$\begin{array}{l}\text { Nitrous ox- } \\
\text { ide alone } \\
\text { versus ni- } \\
\text { trous oxide } \\
\text { with nasal } \\
\text { supplement }\end{array}$} & $\begin{array}{l}\text { Satisfaction with pain relief during the } \\
\text { first stage of labour (considerable to com- } \\
\text { plete pain relief) }\end{array}$ & $1(42)$ & $\begin{array}{l}\text { RR } 1.18,95 \% \mathrm{Cl} 0.94 \text { to } 1.48 \text {, no significant difference } \\
\text { between groups }\end{array}$ \\
\hline & Sense of control in labour & & Outcome not reported \\
\hline & Satisfaction with childbirth experience & & Outcome not reported \\
\hline & Effect on mother/baby interaction & & Outcome not reported \\
\hline & Breastfeeding & & Outcome not reported \\
\hline
\end{tabular}

Pain management for women in labour: an overview of systematic reviews (Review) 
Table 22. (1.) Results by individual review - inhaled analgesia (Continued)

\begin{tabular}{|c|c|c|c|}
\hline & Assisted vaginal birth & & Outcome not reported \\
\hline $\begin{array}{l}\text { Methoxyflu- } \\
\text { rane using } \\
\text { Penthrane } \\
\text { Analgiz- } \\
\text { er versus } \\
\text { methoxyflu- } \\
\text { rane using } \\
\text { Cyprane in- } \\
\text { haler }\end{array}$ & Caesarean section & $1(26)$ & $\begin{array}{l}\mathrm{RR} 2.60,95 \% \mathrm{Cl} 0.12 \text { to } 58.48 \text {, no significant difference } \\
\text { between groups }\end{array}$ \\
\hline
\end{tabular}

\begin{tabular}{|c|c|c|c|}
\hline $\begin{array}{l}\text { Nitrous ox- } \\
\text { ide alone } \\
\text { versus ni- } \\
\text { trous oxide } \\
\text { with nasal } \\
\text { supplement }\end{array}$ & Adverse effects for women (vomiting) & $1(49)$ & $\begin{array}{l}\mathrm{RR} 1.76,95 \% \mathrm{Cl} 0.77 \text { to } 4.00 \text {, more vomiting reported in } \\
\text { the nitrous oxide alone group, although this difference } \\
\text { did not reach statistical significance }\end{array}$ \\
\hline $\begin{array}{l}\text { Methoxyflu- } \\
\text { rane using } \\
\text { Penthrane } \\
\text { Analgiz- } \\
\text { er versus } \\
\text { methoxyflu- } \\
\text { rane using } \\
\text { Cyprane in- } \\
\text { haler }\end{array}$ & Adverse effects for women (vomiting) & $1(26)$ & $\begin{array}{l}\text { Not estimable - no incidence of vomiting in either } \\
\text { group }\end{array}$ \\
\hline
\end{tabular}

\begin{tabular}{|c|c|c|c|}
\hline $\begin{array}{l}\text { Analgiz- } \\
\text { er versus } \\
\text { methoxyflu- } \\
\text { rane using } \\
\text { Cyprane in- } \\
\text { haler }\end{array}$ & $\begin{array}{l}\text { Adverse effects for women (postpartum } \\
\text { haemorrhage) }\end{array}$ & $1(26)$ & $\begin{array}{l}\text { RR } 0.29,95 \% \mathrm{Cl} 0.01 \text { to } 6.50 \text {, no significant difference } \\
\text { between groups }\end{array}$ \\
\hline $\begin{array}{l}\text { Analgiz- } \\
\text { er versus } \\
\text { methoxyflu- } \\
\text { rane using } \\
\text { Cyprane in- } \\
\text { haler }\end{array}$ & $\begin{array}{l}\text { Adverse effects for women (mild pre- } \\
\text { eclampsia) }\end{array}$ & $1(26)$ & $\begin{array}{l}\text { RR } 0.86,95 \% \mathrm{Cl} 0.06 \text { to } 12.28 \text {, no significant difference } \\
\text { between groups }\end{array}$ \\
\hline
\end{tabular}

\begin{tabular}{|c|c|c|c|}
\hline & Adverse effects for infants & & Outcome not reported \\
\hline & $\begin{array}{l}\text { Admission to special care baby unit/ } \\
\text { neonatal intensive care unit }\end{array}$ & & Outcome not reported \\
\hline & $\begin{array}{l}\text { Apgar score less than seven at five min- } \\
\text { utes }\end{array}$ & & $\begin{array}{l}\text { Outcome not in a suitable format (only reported mean } \\
\text { and SD) }\end{array}$ \\
\hline & $\begin{array}{l}\text { Poor infant outcomes at long-term fol- } \\
\text { low-up }\end{array}$ & & Outcome not reported \\
\hline & Cost & & Outcome not reported \\
\hline $\begin{array}{l}\text { 1.3. Inhaled } \\
\text { analgesia } \\
\text { versus TENS }\end{array}$ & Outcome & $\begin{array}{l}\text { No. of stud- } \\
\text { ies (no. } \\
\text { women) }\end{array}$ & Results \\
\hline
\end{tabular}

Pain management for women in labour: an overview of systematic reviews (Review) 
Table 22. (1.) Results by individual review - inhaled analgesia (Continued)

(1 study, 20

women)

\begin{tabular}{|c|c|c|}
\hline $\begin{array}{l}\text { Pain intensity during first stage of labour } \\
\text { (moderate to severe) }\end{array}$ & $1(19)$ & $\begin{array}{l}\mathrm{RR} 1.10,95 \% \mathrm{Cl} 0.84 \text { to } 1.45 \text {, no evidence of a significant } \\
\text { difference between groups }\end{array}$ \\
\hline Satisfaction with pain relief & $1(20)$ & $\begin{array}{l}\text { RR } 0.56,95 \% \mathrm{Cl} 0.29 \text { to } 1.07 \text {, no evidence of a significant } \\
\text { difference between groups }\end{array}$ \\
\hline Sense of control in labour & & Outcome not reported \\
\hline Satisfaction with childbirth experience & & Outcome not reported \\
\hline $\begin{array}{l}\text { Effect (negative) on mother/baby interac- } \\
\text { tion }\end{array}$ & & Outcome not reported \\
\hline Breastfeeding & & Outcome not reported \\
\hline Assisted vaginal birth & & Outcome not reported \\
\hline Caesarean section & & Outcome not reported \\
\hline Adverse effects for women and infants & & Outcome not reported \\
\hline $\begin{array}{l}\text { Admission to special care baby unit/ } \\
\text { neonatal intensive care unit }\end{array}$ & & Outcome not reported \\
\hline $\begin{array}{l}\text { Apgar score less than seven at five min- } \\
\text { utes }\end{array}$ & & Outcome not reported in this format \\
\hline $\begin{array}{l}\text { Poor infant outcomes at long-term fol- } \\
\text { low-up }\end{array}$ & & Outcome not reported \\
\hline Cost & & Outcome not reported \\
\hline
\end{tabular}

Cl: confidence interval; RR: risk ratio

Table 23. (2.) Results by individual review - parenteral opioids versus placebo/IM opioids versus different IM opioids

2. Parenteral opioids for maternal pain management in labour, 57 studies (Ullman 2010)

\begin{tabular}{llll}
\hline $\begin{array}{l}\text { 2.1. Parenteral opioids versus } \\
\text { placebo or no treatment (3 stud- } \\
\text { ies } \mathbf{2 2 6} \text { women) }\end{array}$ & Outcome & $\begin{array}{l}\text { No. of stud- } \\
\text { ies (no. } \\
\text { women) }\end{array}$ & Results \\
\hline IM pethidine versus placebo & $\begin{array}{l}\text { Maternal pain relief } 30 \\
\text { minutes after drug ad- } \\
\text { ministration, defined } \\
\text { as a reduction in visu- } \\
\text { al analogue scale (VAS) } \\
\text { score of at least } 40 \mathrm{~mm}\end{array}$ & $1(50)$ & $\begin{array}{l}\text { RR } 25.00,95 \% \mathrm{Cl} 1.56 \text { to } 400.54, \text { significantly more } \\
\text { women in the pethidine group had a greater reduc- } \\
\text { tion in pain score, Cl very wide }\end{array}$ \\
\hline
\end{tabular}


Table 23. (2.) Results by individual review - parenteral opioids versus placebo/IM opioids versus different IM opioids (Continued)

IM pethidine versus placebo minutes after administration of study drug

Sense of control in

labour

Satisfaction with child-

birth experience

\begin{tabular}{|c|c|c|c|}
\hline & $\begin{array}{l}\text { Effect on mother/baby } \\
\text { interaction }\end{array}$ & & Outcome not reported \\
\hline & Breastfeeding & & Outcome not reported \\
\hline IM pethidine versus placebo & Assisted vaginal birth & $1(50)$ & $\begin{array}{l}\mathrm{RR} 0.86,95 \% \mathrm{Cl} 0.34 \text { to } 2.19 \text {, no evidence of a signif- } \\
\text { icant difference }\end{array}$ \\
\hline IM pethidine versus placebo & Caesarean section & $1(50)$ & $\begin{array}{l}\mathrm{RR} 0.83,95 \% \mathrm{Cl} 0.29 \text { to } 2.38 \text {, no evidence of a signif- } \\
\text { icant difference }\end{array}$ \\
\hline IM pethidine versus placebo & $\begin{array}{l}\text { Adverse effects for } \\
\text { women - Nausea and } \\
\text { vomiting }\end{array}$ & $2(166)$ & $\begin{array}{l}\text { RR } 1.47,95 \% \mathrm{Cl} 0.65 \text { to } 3.31 \text {, no evidence of a signif- } \\
\text { icant difference }\end{array}$ \\
\hline IM pethidine versus placebo & $\begin{array}{l}\text { Adverse effects for in- } \\
\text { fants - Neonatal resus- } \\
\text { citation }\end{array}$ & $1(50)$ & $\begin{array}{l}\text { RR } 1.67,95 \% \mathrm{Cl} 0.45 \text { to } 6.24 \text {, no evidence of a signif- } \\
\text { icant difference }\end{array}$ \\
\hline \multirow[t]{2}{*}{ IM pethidine versus placebo } & $\begin{array}{l}\text { Admission to special } \\
\text { care baby unit/neonatal } \\
\text { intensive care unit }\end{array}$ & $1(50)$ & $\begin{array}{l}\mathrm{RR} 1.00,95 \% \mathrm{Cl} 0.07 \text { to } 15.12 \text {, no evidence of a sig- } \\
\text { nificant difference }\end{array}$ \\
\hline & $\begin{array}{l}\text { Apgar score less than } \\
\text { seven at five minutes }\end{array}$ & & Outcome not reported \\
\hline
\end{tabular}

Poor infant outcomes at

follow-up

Cost

Pain intensity

\subsubsection{IM opioids versus different IM opioids (15 different compar- isons, 36 studies)}

IM meptazinol versus IM pethidine

RR $7.00,95 \% \mathrm{Cl} 0.38$ to 128.87 , no evidence of a significant difference

Outcome not reported

Outcome not reported

utcome not reported

utcome not reported

RR $0.86,95 \% \mathrm{Cl} 0.34$ to 2.19 , no evidence of a signif-

RR $0.83,95 \% \mathrm{Cl} 0.29$ to 2.38 , no evidence of a signif- 
Table 23. (2.) Results by individual review - parenteral opioids versus placebo/IM opioids versus different IM opioids (Continued)

for heterogeneity $P=0.18$ ), no evidence of a significant difference

\section{PCA (IM) meptazinol versus PCA} (IM) pethidine
Pain scores measured one day postpartum

\section{IM diamorphine + prochlor-} perazine versus IM pethidine + prochlorperazine

\section{Global assessment of} pain relief evaluated at 24 hours - rated as 'fair' or 'poor' pain relief
$1(10)$

$1(133)$

MD $-17.60,95 \% \mathrm{Cl}-49.93$ to 14.73 , no evidence of a significant difference

RR $0.88,95 \% \mathrm{Cl} 0.67$ to 1.16 , no evidence of a significant difference

RR $0.85,95 \% \mathrm{Cl} 0.72$ to 1.01 , no evidence of a significant difference

Pain intensity rated as

$1(133)$ moderate or severe one hour after drug administration

\begin{tabular}{|c|c|c|c|}
\hline IM tramadol versus IM pethidine & $\begin{array}{l}\text { Pain intensity (defined } \\
\text { in disparate ways in } \\
\text { studies) - rated as } \\
\text { 'poor' pain relief }\end{array}$ & $4(243)$ & $\begin{array}{l}\mathrm{RR} 1.56,95 \% \mathrm{Cl} 1.10 \text { to } 2.21 \text {, significantly more } \\
\text { women had poor pain relief with tramadol com- } \\
\text { pared with pethidine }\end{array}$ \\
\hline $\begin{array}{l}\text { IM dihydrocodeine versus IM pethi- } \\
\text { dine }\end{array}$ & $\begin{array}{l}\text { Pain intensity rate as } \\
\text { 'poor' pain relief one } \\
\text { hour after drug admin- } \\
\text { istration }\end{array}$ & $1(138)$ & $\begin{array}{l}\text { RR } 1.09,95 \% \mathrm{Cl} 0.64 \text { to } 1.86 \text {, no evidence of a signif- } \\
\text { icant difference }\end{array}$ \\
\hline IM pentazocine versus pethidine & $\begin{array}{l}\text { Pain intensity rated as } \\
\text { 'good' or 'very good' } \\
\text { pain relief at birth }\end{array}$ & $2(253)$ & $\begin{array}{l}\text { RR } 1.08,95 \% \mathrm{Cl} 0.92 \text { to } 1.27 \text {, no evidence of a signif- } \\
\text { icant difference }\end{array}$ \\
\hline \multirow[t]{3}{*}{ IM nalbuphine versus pethidine } & $\begin{array}{l}\text { Number of women re- } \\
\text { ported as being 'free of } \\
\text { pain' }\end{array}$ & $1(40)$ & $\begin{array}{l}\text { RR } 6.00,95 \% \mathrm{Cl} 0.79 \text { to } 45.42 \text {, no evidence of a sig- } \\
\text { nificant difference }\end{array}$ \\
\hline & $\begin{array}{l}\text { Pain intensity at } 30 \text { min- } \\
\text { utes, rated as severe }\end{array}$ & $1(295)$ & $\begin{array}{l}\text { RR } 0.86,95 \% \mathrm{Cl} 0.59 \text { to } 1.26 \text {, no evidence of a signif- } \\
\text { icant difference }\end{array}$ \\
\hline & $\begin{array}{l}\text { Pain intensity at } 60 \text { min- } \\
\text { utes (VAS), at peak of } \\
\text { contraction }\end{array}$ & $1(72)$ & $\begin{array}{l}\mathrm{MD}-8.00,95 \% \mathrm{Cl}-18.55 \text { to } 2.55, \text { no evidence of a } \\
\text { significant difference }\end{array}$ \\
\hline IM morphine versus pethidine & $\begin{array}{l}\text { Number of women rat- } \\
\text { ing their pain relief as } \\
\text { 'poor' }\end{array}$ & $1(90)$ & $\begin{array}{l}\text { RR } 1.22,95 \% \mathrm{Cl} 0.56 \text { to } 2.66 \text {, no evidence of a signif- } \\
\text { icant difference }\end{array}$ \\
\hline $\begin{array}{l}\text { IM pentazocine versus IM pethilor- } \\
\text { fan }\end{array}$ & $\begin{array}{l}\text { Number of women re- } \\
\text { porting 'no pain relief' } \\
\text { at one hour }\end{array}$ & $1(69)$ & $\begin{array}{l}\text { RR } 1.22,95 \% \mathrm{Cl} 0.77 \text { to } 1.95 \text {, no evidence of a signif- } \\
\text { icant difference }\end{array}$ \\
\hline $\begin{array}{l}\text { 2.2.1. IM opioids versus different } \\
\text { IM opioids ( } 15 \text { different compar- } \\
\text { isons, } 36 \text { studies) }\end{array}$ & $\begin{array}{l}\text { Satisfaction with pain } \\
\text { relief }\end{array}$ & $\begin{array}{l}\text { No. of stud- } \\
\text { ies (no. } \\
\text { women) }\end{array}$ & Results \\
\hline $\begin{array}{l}\text { PCA (IM) meptazinol versus PCA } \\
\text { (IM) pethidine }\end{array}$ & $\begin{array}{l}\text { Satisfied with mode of } \\
\text { administration }\end{array}$ & $1(10)$ & $\begin{array}{l}\text { RR } 1.00,95 \% \mathrm{Cl} 0.71 \text { to } 1.41 \text {, no evidence of a signif- } \\
\text { icant difference }\end{array}$ \\
\hline
\end{tabular}


Table 23. (2.) Results by individual review - parenteral opioids versus placebo/IM opioids versus different IM opioids (Continued)

\begin{tabular}{|c|c|c|c|}
\hline IM pentazocine versus pethidine & $\begin{array}{l}\text { Pain relief reported as } \\
\text { "poor" or "partial relief" }\end{array}$ & $3(365)$ & $\begin{array}{l}\text { RR } 1.23,95 \% \mathrm{Cl} 0.74 \text { to } 2.05 \text {, no evidence of a signit } \\
\text { icant difference }\end{array}$ \\
\hline IM nalbuphine versus pethidine & $\begin{array}{l}\text { Maternal satisfaction } \\
\text { with analgesia at } 24 \\
\text { hours; numbers dissat- }\end{array}$ & $1(72)$ & $\begin{array}{l}\text { RR } 0.73,95 \% \mathrm{Cl} 0.55 \text { to } 0.96 \text {, significantly fewer } \\
\text { women in the nalbuphine group were dissatisfied } \\
\text { with pain relief }\end{array}$ \\
\hline
\end{tabular}

\subsubsection{IM opioids versus different IM opioids (15 different compar- isons, 36 studies)}

\subsubsection{IM opioids versus different IM opioids (15 different compar- isons, 36 studies)}

\subsubsection{IM opioids versus different IM opioids (15 different compar- isons, 36 studies)}

\section{Satisfation with child-} birth experience

\section{Outcome not reported}

\section{Sense of control in}

labour

\section{Outcome not reported}

Effect on mother/baby interaction

Outcome not reported

\section{Breastfeeding}

Outcome not reported

\subsubsection{IM opioids versus different IM opioids (15 different compar- isons, 36 studies)}

\subsubsection{IM opioids versus different IM opioids (15 different compar- isons, 36 studies)}

\section{Assisted vaginal birth No. of stud- Results ies (no. women)}

$3(1266)$
RR $1.00,95 \% \mathrm{Cl} 0.81$ to 1.22 , no evidence of a significant difference

\begin{tabular}{|c|c|c|c|}
\hline $\begin{array}{l}\text { Diamorphine + prochloprerazine } \\
\text { versus pethidine + prochlopre- } \\
\text { razine }\end{array}$ & Assisted vaginal birth & $1(133)$ & $\begin{array}{l}\text { RR } 0.96,95 \% \mathrm{Cl} 0.46 \text { to } 2.02 \text {, no evidence of a signif- } \\
\text { icant difference }\end{array}$ \\
\hline IM tramadol versus pethidine & Assisted vaginal birth & $3(260)$ & $\begin{array}{l}\text { RR } 0.56 .95 \% \mathrm{Cl} 0.12 \text { to } 2.56 \text {, no evidence of a signif- } \\
\text { icant difference }\end{array}$ \\
\hline IM pentazocine versus pethidine & Assisted vaginal birth & $1(94)$ & $\begin{array}{l}\mathrm{RR} 5.22,95 \% \mathrm{Cl} 0.63 \text { to } 42.97 \text {, no evidence of a sig- } \\
\text { nificant difference }\end{array}$ \\
\hline IM nalbuphine versus pethidine & Assisted vaginal birth & $2(382)$ & $\begin{array}{l}\text { RR } 0.98,95 \% \mathrm{Cl} 0.25 \text { to } 3.85 \text {, no evidence of a signif- } \\
\text { icant difference }\end{array}$ \\
\hline $\begin{array}{l}\text { IM pentazocine versus IM pethilor- } \\
\text { fan }\end{array}$ & Assisted vaginal birth & $1(98)$ & $\begin{array}{l}\mathrm{RR} 1.04,95 \% \mathrm{Cl} 0.07 \text { to } 16.19 \text {, no evidence of a sig- } \\
\text { nificant difference }\end{array}$ \\
\hline $\begin{array}{l}\text { 2.2.1. IM opioids versus different } \\
\text { IM opioids ( } 15 \text { different compar- } \\
\text { isons, } 36 \text { studies) }\end{array}$ & Caesarean section & $\begin{array}{l}\text { No. of stud- } \\
\text { ies (no. } \\
\text { women) }\end{array}$ & Results \\
\hline IM pethidine versus placebo & Caesarean section & $1(50)$ & $\begin{array}{l}\text { RR } 0.83,95 \% \mathrm{Cl} 0.29 \text { to } 2.38 \text {, no evidence of a signif- } \\
\text { icant difference }\end{array}$ \\
\hline IM meptazinol versus IM pethidine & Caesarean section & $3(1266)$ & $\begin{array}{l}\text { average RR } 0.56,95 \% \mathrm{Cl} 0.16 \text { to } 2.00 \text {, (random ef- } \\
\text { fects; heterogeneity: } \mathrm{I}^{2}=75 \%, \mathrm{Tau}^{2}=0.84, \mathrm{Chi}^{2} \text { test }\end{array}$ \\
\hline
\end{tabular}


Table 23. (2.) Results by individual review - parenteral opioids versus placebo/IM opioids versus different IM opioids (Continued)

for heterogeneity $P=0.02$ ), no evidence of a significant difference

\begin{tabular}{llll}
\hline $\begin{array}{l}\text { Diamorphine + prochloprerazine } \\
\text { versus pethidine + prochlopre- } \\
\text { razine }\end{array}$ & Caesarean section & $1(133)$ & $\begin{array}{l}\text { RR 0.52,95\% Cl 0.10 to 2.76, no evidence of a signif- } \\
\text { icant difference }\end{array}$ \\
\hline IM tramadol versus IM pethidine & Caesarean section & $3(260)$ & $\begin{array}{l}\text { RR } 0.71,95 \% \mathrm{Cl} 0.23 \text { to 2.18, no evidence of a signif- } \\
\text { icant difference }\end{array}$ \\
\hline IM nalbuphine versus IM pethidine & Caesarean section & $1(310)$ & $\begin{array}{l}\text { RR } 0.45,95 \% \mathrm{Cl} 0.12 \text { to } 1.69, \text { no evidence of a signif- } \\
\text { icant difference }\end{array}$ \\
\hline IM Avacan versus IM pentazocine & Caesarean section & $1(184)$ & $\begin{array}{l}\text { RR } 0.63,95 \% \mathrm{Cl} 0.21 \text { to } 1.84, \text { no evidence of a signif- } \\
\text { icant difference }\end{array}$
\end{tabular}

\subsubsection{IM opioids versus different Adverse effects for IM opioids (15 different compar- isons, 36 studies)}

\begin{tabular}{lll}
\hline IM meptazinol versus pethidine & Nausea & $3(1590)$ \\
\hline IM meptazinol versus pethidine & Vomiting & $3(1589)$
\end{tabular}

\section{No. of stud- Results \\ ies (no. \\ women) \\ Adverse
women}

$3(1590)$
RR $1.11,95 \% \mathrm{Cl} 0.95$ to 1.28 , no evidence of a significant difference
IM meptazinol versus pethidine

Maternal sleepiness
$3(1590)$
RR $1.25,95 \% \mathrm{Cl} 1.06$ to 1.47 , significantly more women reported vomiting with meptazinol compared with pethidine

average RR $0.55,95 \% \mathrm{Cl} 0.28$ to 1.07 , (random effects; heterogeneity: $\left.\right|^{2}=44 \%$, Tau $^{2}=0.18$, Chi ${ }^{2}$ test for heterogeneity $\mathrm{P}=0.17$ ), fewer women reported sleepiness in the meptazinol group, although the difference did not reach statistical significance

\begin{tabular}{|c|c|c|c|}
\hline $\begin{array}{l}\text { PCA IM meptazinol versus PCA IM } \\
\text { pethidine }\end{array}$ & $\begin{array}{l}\text { Nausea score in labour } \\
\text { (rated } 1 \text { day after deliv- } \\
\text { ery) }\end{array}$ & $1(10)$ & $\begin{array}{l}\mathrm{MD}-8.00,95 \% \mathrm{Cl}-48.70 \text { to } 32.70 \text {, no evidence of a } \\
\text { significant difference }\end{array}$ \\
\hline $\begin{array}{l}\text { PCA IM meptazinol versus PCA IM } \\
\text { pethidine }\end{array}$ & $\begin{array}{l}\text { Drowsiness score in } \\
\text { labour (rated } 1 \text { day af- } \\
\text { ter delivery) }\end{array}$ & $1(10)$ & $\begin{array}{l}\text { MD } 5.60,95 \% \mathrm{Cl}-28.19 \text { to } 39.39 \text {, no evidence of a } \\
\text { significant difference }\end{array}$ \\
\hline $\begin{array}{l}\text { IM diamorphine + prochlor- } \\
\text { perazine versus IM pethidine + } \\
\text { prochlorperazine }\end{array}$ & Vomiting & $1(133)$ & $\begin{array}{l}\mathrm{RR} 0.39,95 \% \mathrm{Cl} 0.17 \text { to } 0.86 \text {, number of women } \\
\text { vomiting significantly lower with diamorphine } \\
\text { compared with pethidine }\end{array}$ \\
\hline $\begin{array}{l}\text { IM diamorphine + prochlor- } \\
\text { perazine versus IM pethidine + } \\
\text { prochlorperazine }\end{array}$ & $\begin{array}{l}\text { Maternal sleepiness } \\
\text { (one hour after study } \\
\text { drug administration) }\end{array}$ & $1(133)$ & $\begin{array}{l}\text { RR } 0.93,95 \% \mathrm{Cl} 0.52 \text { to } 1.66 \text {, no evidence of a signif- } \\
\text { icant difference }\end{array}$ \\
\hline IM tramadol versus pethidine & Nausea and vomiting & $6(454)$ & $\begin{array}{l}\text { Average RR } 0.97,95 \% \mathrm{Cl} 0.34 \text { to } 2.76 \text {, (random ef- } \\
\text { fects; heterogeneity: } \mathrm{I}^{2}=72 \% \text {, Tau }{ }^{2}=1.09, \mathrm{Chi}^{2} \text { test } \\
\text { for heterogeneity } \mathrm{P}=0.003 \text { ), no evidence of a sig- } \\
\text { nificant difference }\end{array}$ \\
\hline IM tramadol versus pethidine & Maternal sleepiness & $5(409)$ & $\begin{array}{l}\text { RR } 0.57,95 \% \mathrm{Cl} 0.33 \text { to } 0.97 \text {, (random effects; het- } \\
\text { erogeneity: } I^{2}=72 \%, \mathrm{Tau}^{2}=0.24, \mathrm{Chi}^{2} \text { test for het- }\end{array}$ \\
\hline
\end{tabular}


Table 23. (2.) Results by individual review - parenteral opioids versus placebo/IM opioids versus different IM opioids (Continued)

erogeneity $\mathrm{P}=0.007$ ), significantly fewer women in the tramadol group reported sleepiness

\begin{tabular}{llll}
\hline $\begin{array}{l}\text { IM tramadol + triflupromazine ver- } \\
\text { sus pethidine + triflupromazine }\end{array}$ & Nausea & $1(40)$ & $\begin{array}{l}\text { RR } 0.82,95 \% \mathrm{Cl} 0.13 \text { to } 5.25, \text { no evidence of a signif- } \\
\text { icant difference }\end{array}$ \\
\hline $\begin{array}{l}\text { IM tramadol + triflupromazine ver- } \\
\text { sus pethidine + triflupromazine }\end{array}$ & Vomiting & $1(40)$ & $\begin{array}{l}\text { RR } 0.40,95 \% \mathrm{Cl} 0.02 \text { to } 9.35, \text { no evidence of a signif- } \\
\text { icant difference }\end{array}$ \\
\hline $\begin{array}{l}\text { IM tramadol + triflupromazine ver- } \\
\text { sus pethidine + triflupromazine }\end{array}$ & Maternal sleepiness & $1(40)$ & $\begin{array}{l}\text { RR } 2.86,95 \% \mathrm{Cl} 0.68 \text { to } 12.12, \text { sleepiness was more } \\
\text { frequently reported by women who received tra- } \\
\text { madol, though no statistically significant difference } \\
\text { between groups was detected }\end{array}$ \\
\hline
\end{tabular}

\begin{tabular}{llll}
\hline $\begin{array}{l}\text { IM dihydrocodeine versus IM pethi- } \\
\text { dine }\end{array}$ & Nausea and vomiting & $1(138)$ & $\begin{array}{l}\text { RR } 0.87,95 \% \mathrm{Cl} 0.40 \text { to } 1.88, \text { no evidence of a signif- } \\
\text { icant difference }\end{array}$ \\
\hline $\begin{array}{l}\text { IM dihydrocodeine versus IM pethi- } \\
\text { dine }\end{array}$ & Maternal sleepiness & $1(138)$ & $\begin{array}{l}\text { RR } 0.67,95 \% \mathrm{Cl} 0.43 \text { to } 1.04, \text { no evidence of a signif- } \\
\text { icant difference }\end{array}$ \\
\hline
\end{tabular}

\begin{tabular}{l}
\hline IM pentazocine versus IM pethi- Nausea \\
dine
\end{tabular}

RR $0.46,95 \% \mathrm{Cl} 0.24$ to 0.90 , fewer women in the pentazocine group reported nausea compared to the pethidine group

\begin{tabular}{lll}
\hline $\begin{array}{l}\text { IM pentazocine versus IM pethi- } \\
\text { dine }\end{array}$ & Vomiting & $1(73)$ \\
\hline $\begin{array}{l}\text { IM pentazocine versus IM pethi- } \\
\text { dine }\end{array}$ & Maternal sleepiness & $3(391)$ \\
\hline IM nalbuphine versus IM pethidine & Nausea & $1(301)$
\end{tabular}
RR $0.92,95 \% \mathrm{Cl} 0.27$ to 3.14 , no evidence of a significant difference

RR $1.00,95 \% \mathrm{Cl} 0.89$ to 1.12 , no evidence of a significant difference

RR $0.62,95 \% \mathrm{Cl} 0.42$ to 0.91 , fewer women in the nalbuphine group reported nausea compared to the pethidine group

IM nalbuphine versus IM pethidine Vomiting $1(301)$

RR $0.41,95 \% \mathrm{Cl} 0.22$ to 0.76 , fewer women in the nalbuphine group reported vomiting compared to the pethidine group

IM nalbuphine versus IM pethidine Nausea and vomiting $1(72)$

RR $0.41,95 \% \mathrm{Cl} 0.18$ to 0.94 , fewer women in the nalbuphine group reported both nausea and vomiting compared to the pethidine group

\begin{tabular}{|c|c|c|c|}
\hline IM nalbuphine versus IM pethidine & Sleepiness & $1(72)$ & $\begin{array}{l}\text { RR } 3.78,95 \% \mathrm{Cl} 0.86 \text { to } 16.60 \text {, no evidence of a sig- } \\
\text { nificant difference }\end{array}$ \\
\hline $\begin{array}{l}\text { IM phenazocine versus IM pethi- } \\
\text { dine }\end{array}$ & Vomiting & $1(212)$ & $\begin{array}{l}\mathrm{RR} 0.39,95 \% \mathrm{Cl} 0.20 \text { to } 0.78 \text {, fewer women in the } \\
\text { phenazocine group reported vomiting compared to } \\
\text { the pethidine group }\end{array}$ \\
\hline IM morphine versus IM pethidine & Nausea and vomiting & $1(90)$ & $\begin{array}{l}\text { RR } 1.00,95 \% \mathrm{Cl} 0.21 \text { to } 4.69 \text {, no evidence of a signif- } \\
\text { icant difference }\end{array}$ \\
\hline IM morphine versus IM pethidine & Maternal sleepiness & $1(90)$ & $\begin{array}{l}\text { RR } 0.60,95 \% \mathrm{Cl} 0.29 \text { to } 1.23 \text {, no evidence of a signif- } \\
\text { icant difference }\end{array}$ \\
\hline $\begin{array}{l}\text { IM butorphanol versus IM pethi- } \\
\text { dine }\end{array}$ & Nausea & $1(80)$ & $\begin{array}{l}\text { RR } 0.20,95 \% \mathrm{Cl} 0.01 \text { to } 4.04 \text {, no evidence of a signif- } \\
\text { icant difference }\end{array}$ \\
\hline
\end{tabular}

Pain management for women in labour: an overview of systematic reviews (Review) Copyright $\odot 2013$ The Cochrane Collaboration. Published by John Wiley \& Sons, Ltd. 
Table 23. (2.) Results by individual review - parenteral opioids versus placebo/IM opioids versus different IM opioids (Continued)

\begin{tabular}{llll}
$\begin{array}{l}\text { IM butorphanol versus IM pethi- } \\
\text { dine }\end{array}$ & Vomiting & $1(80)$ & $\begin{array}{l}\text { RR 0.50, 95\% } \mathrm{Cl} 0.05 \text { to } 5.30, \text { no evidence of a signif- } \\
\text { icant difference }\end{array}$ \\
\hline IM tramadol versus no treatment & $\begin{array}{l}\text { Mean blood loss at de- } \\
\text { livery }(\mathrm{ml})\end{array}$ & $1(60)$ & $\begin{array}{l}\text { MD } 25.70,95 \% \mathrm{Cl}-9.83 \text { to } 61.23, \text { no evidence of a } \\
\text { significant difference }\end{array}$
\end{tabular}

\section{$\begin{array}{lll}\begin{array}{l}\text { 2.2.1. IM opioids versus different } \\ \text { IM opioids (15 different compar- }\end{array} & \begin{array}{l}\text { Adverse effects for in- } \\ \text { fants }\end{array} & \begin{array}{l}\text { No. of stud- } \\ \text { ies (no. }\end{array}\end{array} \quad \begin{aligned} & \text { Results } \\ & \end{aligned}$

isons, 36 studies) women)

\begin{tabular}{llll}
\hline IM meptazinol versus pethidine & $\begin{array}{l}\text { Fetal heart rate changes } \\
\text { (decelerations) }\end{array}$ & $1(34)$ & $\begin{array}{l}\text { RR 1.23, 95\% Cl 0.92 to 1.64, no evidence of a signif- } \\
\text { icant difference }\end{array}$ \\
\hline IM meptazinol versus pethidine & $\begin{array}{l}\text { Naloxone administra- } \\
\text { tion }\end{array}$ & $1(998)$ & $\begin{array}{l}\text { RR 0.89, 95\% Cl 0.77 to 1.02, no evidence of a signif- } \\
\text { icant difference }\end{array}$ \\
\hline IM meptazinol versus pethidine & Neonatal resuscitation & $2(1356)$ & $\begin{array}{l}\text { RR } 1.00,95 \% \mathrm{Cl} 0.95 \text { to 1.05, no evidence of a signif- } \\
\text { icant difference }\end{array}$ \\
\hline $\begin{array}{l}\text { PCA IM meptazinol versus PCA IM } \\
\text { pethidine }\end{array}$ & $\begin{array}{l}\text { Naloxone administra- } \\
\text { tion }\end{array}$ & $1(10)$ & $\begin{array}{l}\text { RR } 1.00,95 \% \mathrm{Cl} 0.08 \text { to } 11.93 \text {, no evidence of a sig- } \\
\text { nificant difference }\end{array}$ \\
\hline $\begin{array}{l}\text { IM diamorphine + prochlorpre- } \\
\text { razine versus IM pethidine }+ \\
\text { prochlorprerazine }\end{array}$ & Neonatal resuscitation & $1(133)$ & $\begin{array}{l}\text { RR } 1.21,95 \% \mathrm{Cl} 0.73 \text { to 2.02, no evidence of a signif- } \\
\text { icant difference }\end{array}$
\end{tabular}

\begin{tabular}{|c|c|c|c|}
\hline IM tramadol versus pethidine & $\begin{array}{l}\text { Neonatal respiratory } \\
\text { distress }\end{array}$ & $1(59)$ & $\begin{array}{l}\mathrm{RR} 2.26,95 \% \mathrm{Cl} 0.64 \text { to } 7.89 \text {, no evidence of a signif- } \\
\text { icant difference }\end{array}$ \\
\hline $\begin{array}{l}\text { IM pentazocine + promazine ver- } \\
\text { sus IM pethidine + promazine }\end{array}$ & $\begin{array}{l}\text { Naloxone administra- } \\
\text { tion }\end{array}$ & $1(85)$ & $\begin{array}{l}\text { RR } 0.49,95 \% \mathrm{Cl} 0.09 \text { to } 2.53 \text {, no evidence of a signif- } \\
\text { icant difference }\end{array}$ \\
\hline IM nalbuphine versus IM pethidine & $\begin{array}{l}\text { Naloxone administra- } \\
\text { tion }\end{array}$ & $1(72)$ & $\begin{array}{l}\text { RR } 6.63,95 \% \mathrm{Cl} 0.35 \text { to } 123.93 \text {, no evidence of a sig- } \\
\text { nificant difference }\end{array}$ \\
\hline IM nalbuphine versus IM pethidine & $\begin{array}{l}\text { Neonatal neuro-behav- } \\
\text { ioural 2-4 hours postna- } \\
\text { tal }\end{array}$ & $1(72)$ & $\begin{array}{l}\mathrm{MD}-3.70,95 \% \mathrm{Cl}-6.14 \text { to }-1.26 \text {, babies of women } \\
\text { who received nalbuphine had lower scores than } \\
\text { babies born to women in the pethidine group }\end{array}$ \\
\hline $\begin{array}{l}\text { IM butorphanol versus IM pethi- } \\
\text { dine }\end{array}$ & Neonatal resuscitation & $1(80)$ & $\begin{array}{l}\text { RR } 0.33,95 \% \mathrm{Cl} 0.01 \text { to } 7.95 \text {, no evidence of a signif- } \\
\text { icant difference }\end{array}$ \\
\hline $\begin{array}{l}\text { IM butorphanol versus IM pethi- } \\
\text { dine }\end{array}$ & $\begin{array}{l}\text { Naloxone administra- } \\
\text { tion }\end{array}$ & $1(80)$ & $\begin{array}{l}\text { RR } 0.33,95 \% \mathrm{Cl} 0.01 \text { to } 7.95 \text {, no evidence of a signif- } \\
\text { icant difference }\end{array}$ \\
\hline $\begin{array}{l}\text { 2.2.1. IM opioids versus different } \\
\text { IM opioids (15 different compar- } \\
\text { isons, } 36 \text { studies) }\end{array}$ & $\begin{array}{l}\text { Admission to special } \\
\text { care baby unit }\end{array}$ & $\begin{array}{l}\text { No. of stud- } \\
\text { ies (no. } \\
\text { women) }\end{array}$ & Results \\
\hline IM meptazinol versus pethidine & & $1(199)$ & $\begin{array}{l}\text { RR } 0.88,95 \% \mathrm{Cl} 0.48 \text { to } 1.63 \text {, no evidence of a signif- } \\
\text { icant difference }\end{array}$ \\
\hline $\begin{array}{l}\text { IM diamorphine + prochlorpre- } \\
\text { razine versus IM pethidine + } \\
\text { prochlorprerazine }\end{array}$ & & $1(133)$ & $\begin{array}{l}\text { RR } 0.58,95 \% \mathrm{Cl} 0.21 \text { to } 1.64 \text {, no evidence of a signif- } \\
\text { icant difference }\end{array}$ \\
\hline
\end{tabular}


Table 23. (2.) Results by individual review - parenteral opioids versus placebo/IM opioids versus different IM opioids (Continued)

\begin{tabular}{lll} 
IM tramadol versus IM pethidine & $1(59)$ & $\begin{array}{l}\text { RR } 2.26,95 \% \mathrm{Cl} 0.64 \text { to } 7.89, \text { no evidence of a signif- } \\
\text { icant difference }\end{array}$ \\
\hline IM nalbuphine versus IM pethidine & $1(299)$ & $\begin{array}{l}\text { RR } 1.07,95 \% \mathrm{Cl} 0.61 \text { to } 1.89, \text { no evidence of a signif- } \\
\text { icant difference }\end{array}$
\end{tabular}

\begin{tabular}{|c|c|c|c|}
\hline $\begin{array}{l}\text { 2.2.1. IM opioids versus different } \\
\text { IM opioids (15 different compar- } \\
\text { isons, } 36 \text { studies) }\end{array}$ & $\begin{array}{l}\text { Apgar score less than } \\
\text { seven at five minutes }\end{array}$ & $\begin{array}{l}\text { No. of stud- } \\
\text { ies (no. } \\
\text { women) }\end{array}$ & Results \\
\hline IM meptazinol versus IM pethidine & & $3(616)$ & $\begin{array}{l}\text { RR } 0.49,95 \% \mathrm{Cl} 0.05 \text { to } 5.37 \text {, no evidence of a signif- } \\
\text { icant difference }\end{array}$ \\
\hline $\begin{array}{l}\text { IM diamorphine + prochlorpre- } \\
\text { razine versus IM pethidine + } \\
\text { prochlorprerazine }\end{array}$ & & $1(133)$ & $\begin{array}{l}\text { RR } 0.35,95 \% \mathrm{Cl} 0.04 \text { to } 3.27 \text {, no evidence of a signif- } \\
\text { icant difference }\end{array}$ \\
\hline $\begin{array}{l}\text { IM pentazocine versus IM pethi- } \\
\text { dine }\end{array}$ & & $1(62)$ & $\begin{array}{l}\text { RR } 0.23,95 \% \mathrm{Cl} 0.01 \text { to } 4.54 \text {, no evidence of a signif- } \\
\text { icant difference }\end{array}$ \\
\hline IM nalbuphine versus IM pethidine & & $1(72)$ & $\begin{array}{l}\text { RR } 0.47,95 \% \mathrm{Cl} 0.04 \text { to } 4.99 \text {, no evidence of a signif- } \\
\text { icant difference }\end{array}$ \\
\hline
\end{tabular}

\subsubsection{IM opioids versus different Poor infant outcomes IM opioids (15 different compar- at follow-up isons, 36 studies)}

Outcome not reported

\subsubsection{IM opioids versus different IM opioids (15 different compar- isons, 36 studies)

12 values reported in table only when random effects analysis has been carried out due to substantial heterogeneity indicated by an $\mathrm{I}^{2}$ value greater than $30 \%$

$\mathrm{Cl}$ : confidence interval; IML intramuscular; MD: mean difference; RR: risk ratio

Table 24. (2.) Results by individual review - parenteral opioids - IV opioids versus different IV opioids/parenteral opioids versus different intervention

\footnotetext{
Parenteral opioids for maternal pain management in labour, 57 studies (Ullman 2010)
}

\begin{tabular}{llll}
\hline $\begin{array}{l}\text { 2.2.2. IV opioids versus different IV } \\
\text { opioids (12 comparisons, 17 studies) }\end{array}$ & Pain intensity & $\begin{array}{l}\text { No. of stud- } \\
\text { ies (no. } \\
\text { women) }\end{array}$ & Results \\
\hline IV fentanyl versus IV pethidine & $\begin{array}{l}\text { Mean pain score one hour } \\
\text { after drug administration }\end{array}$ & $1(105)$ & $\begin{array}{l}\mathrm{MD}-0.20,95 \% \mathrm{Cl}-0.34 \text { to }-0.06, \text { the mean } \\
\text { pain score in the IV fentanyl group was sig- } \\
\text { nificantly lower than the mean pain score } \\
\text { in the IV pethidine group }\end{array}$ \\
\hline IV butorphanol versus IV pethidine & $\begin{array}{l}\text { Women's reported mean } \\
\text { pain relief score (not de- } \\
\text { fined) }\end{array}$ & 1 (80) & $\begin{array}{l}\text { MD 0.67, } 95 \% \mathrm{Cl} 0.25 \text { to } 1.09, \text { the mean pain } \\
\text { relief score was significantly higher for the } \\
\text { IV butorphanol group compared to the IV } \\
\text { pethidine group }\end{array}$ \\
\hline
\end{tabular}


Table 24. (2.) Results by individual review - parenteral opioids - IV opioids versus different IV opioids/parenteral opioids versus different intervention (Continued)

\begin{tabular}{|c|c|c|c|}
\hline IV butorphanol versus IV pethidine & $\begin{array}{l}\text { Pain score one hour after } \\
\text { drug administration (10- } \\
\text { point scale) }\end{array}$ & $1(80)$ & $\begin{array}{l}\text { MD }-0.60,95 \% \mathrm{Cl}-1.02 \text { to }-0.18 \text {, the mean } \\
\text { pain score in the IV butorphanol group } \\
\text { was significantly lower than the mean pain } \\
\text { score in the IV pethidine group }\end{array}$ \\
\hline PCA pentazocine versus PCA pethidine & $\begin{array}{l}\text { Self-reported pain score } \\
\text { during labour }\end{array}$ & $1(23)$ & $\begin{array}{l}\text { SMD }-0.76,95 \% \mathrm{Cl}-1.62 \text { to } 0.09 \text {, no evi- } \\
\text { dence of a significant difference }\end{array}$ \\
\hline PCA remifentanil versus PCA pethidine & $\begin{array}{l}\text { Mean pain scores at one } \\
\text { hour after drug adminis- } \\
\text { tration (a visual analogue } \\
\text { scale (VAS) ranging from } \\
0 \text { ("no pain") to } 10 \mathrm{~cm} \\
\text { ("worst imaginable pain") }\end{array}$ & $2(122)$ & $\begin{array}{l}\text { average } \mathrm{MD}-8.59,95 \% \mathrm{Cl}-27.61 \text { to } 10.44 \\
\text { (random effects; heterogeneity: } \mathrm{I}^{2}=62 \% \text {, } \\
\mathrm{T}^{2}=136.73 \text {, Chi } 2 \text { test for heterogeneity } \mathrm{P} \\
=0.10 \text { ), no evidence of a significant differ- } \\
\text { ence }\end{array}$ \\
\hline PCA nalbuphine versus PCA pethidine & $\begin{array}{l}\text { Pain score recorded in } \\
\text { labour }\end{array}$ & $1(60)$ & $\begin{array}{l}\text { SMD }-0.51,95 \% \mathrm{Cl}-1.02 \text { to } 0.00, \text { no evi- } \\
\text { dence of a significant difference }\end{array}$ \\
\hline PCA fentanyl versus PCA alftentanil & $\begin{array}{l}\text { Mean pain scores at } 4-6 \mathrm{~cm} \\
\text { cervical dilatation }\end{array}$ & $1(21)$ & $\begin{array}{l}\mathrm{MD}-12.80,95 \% \mathrm{Cl}-32.12 \text { to } 6.52 \text {, no evi- } \\
\text { dence of a significant difference }\end{array}$ \\
\hline PCA fentanyl versus PCA pethidine & $\begin{array}{l}\text { Mean pain scores at one } \\
\text { hour after drug adminis- } \\
\text { tration (a visual analogue } \\
\text { scale (VAS) ranging from } \\
0 \text { ("no pain") to } 10 \mathrm{~cm} \\
\text { ("worst imaginable pain") }\end{array}$ & $1(107)$ & $\begin{array}{l}\mathrm{MD}-0.65,95 \% \mathrm{Cl}-1.56 \text { to } 0.26 \text {, no evidence } \\
\text { of a significant difference }\end{array}$ \\
\hline
\end{tabular}

\subsubsection{IV opioids versus different IV opioids (12 comparisons, 17 studies)}

IV phenazocine versus IV pethidine

IV phenazocine versus IV pethidine

IV morphine versus IV pethidine

V morphine versus IV pethidine

PCA nalbuphine versus PCA pethidine

PCA fentanyl versus PCA alftentanil

\section{Satisfaction with pain re- lief}

\section{No. of stud- Results \\ ies (no.}

women)

$\begin{array}{lll}\begin{array}{l}\text { Satisfaction with pain re- } \\ \text { lief (women with fair or }\end{array} & 1(194) & \begin{array}{l}\text { RR } 0.72,95 \% \mathrm{Cl} 0.48 \text { to } 1.10, \text { no evidence of } \\ \text { a significant difference }\end{array}\end{array}$
poor relief)
$1(141)$

RR $0.87,95 \% \mathrm{Cl} 0.78$ to 0.98 , significantly fewer women in the IV morphine group were satisfied with pain relief

RR $1.29,95 \% \mathrm{Cl} 0.88$ to 1.89 , no evidence of a significant difference
Measured in the postnatal $1(60)$ period (rated good or excellent) Satisfaction with pain relief as "adequate" or "good" within 6 hours of giving birth
RR $1.56,95 \% \mathrm{Cl} 0.93$ to 2.60 , no evidence of a significant difference

\section{Sense of control in}

labour
Outcome not reported

\subsubsection{IV opioids versus different IV opioids (12 comparisons, 17 studies)}

\section{Satisfaction with child- birth experience}

No. of stud- Results

ies (no.

women) 
Table 24. (2.) Results by individual review - parenteral opioids - IV opioids versus different IV opioids/parenteral opioids versus different intervention (Continued)

\begin{tabular}{|c|c|c|c|}
\hline PCA remifentanil versus PCA pethidine & $\begin{array}{l}\text { Satisfaction with child- } \\
\text { birth experience }\end{array}$ & $1(68)$ & $\begin{array}{l}\text { MD } 1.10,95 \% \mathrm{Cl} 0.46 \text { to } 1.74 \text {, no evidence } \\
\text { of a significant difference }\end{array}$ \\
\hline $\begin{array}{l}\text { 2.2.2. IV opioids versus different IV } \\
\text { opioids ( } 12 \text { comparisons, } 17 \text { studies) }\end{array}$ & $\begin{array}{l}\text { Effect on mother/baby } \\
\text { interaction }\end{array}$ & & Outcome not reported \\
\hline $\begin{array}{l}\text { 2.2.2. IV opioids versus different IV } \\
\text { opioids ( } 12 \text { comparisons, } 17 \text { studies) }\end{array}$ & Breastfeeding & $\begin{array}{l}\text { No. of stud- } \\
\text { ies (no. } \\
\text { women) }\end{array}$ & Results \\
\hline PCA pentazocine versus PCA pethidine & Breastfeeding at discharge & $1(23)$ & $\begin{array}{l}\text { RR } 1.00,95 \% \mathrm{Cl} 0.85 \text { to } 1.17 \text {, no evidence of } \\
\text { a significant difference between groups }\end{array}$ \\
\hline $\begin{array}{l}\text { 2.2.2. IV opioids versus different IV } \\
\text { opioids ( } 12 \text { comparisons, } 17 \text { studies) }\end{array}$ & Assisted vaginal birth & $\begin{array}{l}\text { No. of stud- } \\
\text { ies (no. } \\
\text { women) }\end{array}$ & Results \\
\hline IV butorphanol versus IV pethidine & Assisted vaginal birth & $1(200)$ & $\begin{array}{l}\mathrm{RR} 1.30,95 \% \mathrm{Cl} 0.60 \text { to } 2.83 \text {, no evidence of } \\
\text { a significant difference }\end{array}$ \\
\hline PCA remifentanil versus PCA pethidine & Assisted vaginal birth & $2(97)$ & $\begin{array}{l}\mathrm{RR} 0.96,95 \% \mathrm{Cl} 0.46 \text { to } 2.00 \text {, no evidence of } \\
\text { a significant difference }\end{array}$ \\
\hline PCA fentanyl versus PCA pethidine & Assisted vaginal birth & $1(81)$ & $\begin{array}{l}\mathrm{RR} 0.57,95 \% \mathrm{Cl} 0.22 \text { to } 1.49 \text {, no evidence of } \\
\text { a significant difference }\end{array}$ \\
\hline $\begin{array}{l}\text { 2.2.2. IV opioids versus different IV } \\
\text { opioids ( } 12 \text { comparisons, } 17 \text { studies) }\end{array}$ & Caesarean Section & $\begin{array}{l}\text { No. of stud- } \\
\text { ies (no. } \\
\text { women) }\end{array}$ & Results \\
\hline IV fentanyl versus IV pethidine & Caesarean section & $1(105)$ & $\begin{array}{l}\text { RR } 1.14,95 \% \mathrm{Cl} 0.24 \text { to } 5.40 \text {, no evidence of } \\
\text { a significant difference }\end{array}$ \\
\hline IV nalbuphine versus IV pethidine & Caesarean section & $1(28)$ & $\begin{array}{l}\text { RR } 5.00,95 \% \mathrm{Cl} 0.26 \text { to } 95.61 \text {, no evidence } \\
\text { of a significant difference }\end{array}$ \\
\hline IV butorphanol versus IV pethidine & Caesarean section & $1(200)$ & $\begin{array}{l}\mathrm{RR} 0.80,95 \% \mathrm{Cl} 0.22 \text { to } 2.89 \text {, no evidence of } \\
\text { a significant difference }\end{array}$ \\
\hline IV fentanyl versus IV butorphanol & Caesarean section & $1(100)$ & $\begin{array}{l}\mathrm{RR} 0.80,95 \% \mathrm{Cl} 0.23 \text { to } 2.81 \text {, no evidence of } \\
\text { a significant difference }\end{array}$ \\
\hline PCA pentazocine versus PCA pethidine & Caesarean section & $1(29)$ & $\begin{array}{l}\mathrm{RR} 0.36,95 \% \mathrm{Cl} 0.02 \text { to } 8.07 \text {, no evidence of } \\
\text { a significant difference }\end{array}$ \\
\hline PCA remifentanil versus PCA pethidine & Caesarean section & $2(97)$ & $\begin{array}{l}\text { RR } 1.81,95 \% \mathrm{Cl} 0.60 \text { to } 5.46 \text {, no evidence of } \\
\text { a significant difference }\end{array}$ \\
\hline PCA fentanyl versus PCA alftentanil & Caesarean section & $1(23)$ & $\begin{array}{l}\mathrm{RR} 1.64,95 \% \mathrm{Cl} 0.33 \text { to } 8.03 \text {, no evidence of } \\
\text { a significant difference }\end{array}$ \\
\hline PCA fentanyl versus PCA pethidine & Caesarean section & $1(81)$ & $\begin{array}{l}\mathrm{RR} 0.25,95 \% \mathrm{Cl} 0.03 \text { to } 2.34 \text {, no evidence of } \\
\text { a significant difference }\end{array}$ \\
\hline
\end{tabular}


Table 24. (2.) Results by individual review - parenteral opioids - IV opioids versus different IV opioids/parenteral opioids versus different intervention (Continued)

\subsubsection{IV opioids versus different IV opioids (12 comparisons, 17 studies) \\ Adverse effects for women}

\author{
No. of stud- Results \\ ies (no. \\ women)
}

$\begin{array}{lll}\text { IV fentanyl versus IV pethidine } \quad \text { Nausea and/or vomiting } & 1(105) & \begin{array}{l}\text { RR } 0.51,95 \% \mathrm{Cl} 0.17 \text { to } 1.55, \text { no evidence of } \\ \text { a significant difference }\end{array}\end{array}$

IV fentanyl versus IV pethidine Maternal sedation $\quad$ M (105)

RR $0.05,95 \% \mathrm{Cl} 0.00$ to 0.82 , maternal sedation was significantly lower in women allocated to the IV fentanyl group

\begin{tabular}{llll}
\hline IV phenazocine versus IV pethidine & Nausea and vomiting & $1(194)$ & $\begin{array}{l}\text { RR 0.40,95\% Cl 0.08 to 2.01, no evidence of } \\
\text { a significant difference }\end{array}$ \\
\hline IV butorphanol versus IV pethidine & Nausea and/or vomiting & $1(200)$ & $\begin{array}{l}\text { RR 0.04, 95\% } \mathrm{Cl} 0.00 \text { to } 0.67, \text { fewer women } \\
\text { in the butorphanol group experienced } \\
\text { nausea/and or vomiting compared to the } \\
\text { pethidine group }\end{array}$
\end{tabular}

\begin{tabular}{|c|c|c|c|}
\hline IV morphine versus IV pethidine & Nausea & $1(20)$ & $\begin{array}{l}\mathrm{RR} 0.17,95 \% \mathrm{Cl} 0.02 \text { to } 1.14 \text {, no evidence of } \\
\text { a significant difference }\end{array}$ \\
\hline IV morphine versus IV pethidine & Vomiting & $1(20)$ & $\begin{array}{l}\mathrm{RR} 0.25,95 \% \mathrm{Cl} 0.03 \text { to } 1.86 \text {, no evidence of } \\
\text { a significant difference }\end{array}$ \\
\hline IV nisentil versus IV pethidine & Nausea & $1(395)$ & $\begin{array}{l}\mathrm{RR} 0.71,95 \% \mathrm{Cl} 0.33 \text { to } 1.52 \text {, no evidence of } \\
\text { a significant difference }\end{array}$ \\
\hline IV nisentil versus IV pethidine & Vomiting & $1(395)$ & $\begin{array}{l}\mathrm{RR} 0.38,95 \% \mathrm{Cl} 0.22 \text { to } 0.66 \text {, fewer women } \\
\text { in the nisentil group experienced vomiting } \\
\text { compared to the pethidine group }\end{array}$ \\
\hline PCA pentazocine versus PCA pethidine & Nausea and vomiting & $1(29)$ & $\begin{array}{l}\mathrm{RR} 0.10,95 \% \mathrm{Cl} 0.01 \text { to } 1.61 \text {, no evidence of } \\
\text { a significant difference }\end{array}$ \\
\hline PCA remifentanil versus PCA pethidine & $\begin{array}{l}\text { Maternal sleepiness (as- } \\
\text { sessed using an observer } \\
\text { sedation score: } 1 \text { = awake; } \\
2 \text { = sleepy; } 3 \text { = eyes closed, } \\
\text { but rousable; } 4 \text { = eyes } \\
\text { closed but rousable by } \\
\text { physical stimuli; } 5 \text { = un- } \\
\text { rousable) }\end{array}$ & $1(105)$ & $\begin{array}{l}\text { MD } 0.40,95 \% \mathrm{Cl} 0.14 \text { to } 0.66 \text {, no evidence } \\
\text { of a significant difference between groups }\end{array}$ \\
\hline PCA remifentanil versus PCA pethidine & Nausea and vomiting & $2(119)$ & $\begin{array}{l}\text { RR } 0.95,95 \% \mathrm{Cl} 0.61 \text { to } 1.49 \text {, no evidence of } \\
\text { a significant difference }\end{array}$ \\
\hline PCA nalbuphine versus PCA pethidine & Nausea and vomiting & $1(59)$ & $\begin{array}{l}\text { RR } 0.68,95 \% \mathrm{Cl} 0.30 \text { to } 1.54 \text {, no evidence of } \\
\text { a significant difference }\end{array}$ \\
\hline PCA fentanyl versus PCA alftentanil & Nausea & $1(23)$ & $\begin{array}{l}\mathrm{RR} 2.73,95 \% \mathrm{Cl} 0.66 \text { to } 11.30 \text {, no evidence } \\
\text { of a significant difference }\end{array}$ \\
\hline PCA fentanyl versus PCA pethidine & Maternal sleepiness & $1(107)$ & $\begin{array}{l}\mathrm{MD}-0.06,95 \% \mathrm{Cl}-0.25 \text { to } 0.13 \text {, no evidence } \\
\text { of a significant difference }\end{array}$ \\
\hline
\end{tabular}


Table 24. (2.) Results by individual review - parenteral opioids - IV opioids versus different IV opioids/parenteral opioids versus different intervention (Continued)

$\begin{array}{lll}\text { PCA fentanyl versus PCA pethidine } \quad \text { Nausea and vomiting } & 1(102) & \begin{array}{l}\text { RR } 0.87,95 \% \mathrm{Cl} 0.55 \text { to } 1.37, \text { no evidence of } \\ \text { a significant difference }\end{array}\end{array}$

\begin{tabular}{|c|c|c|c|}
\hline $\begin{array}{l}\text { 2.2.2. IV opioids versus different IV } \\
\text { opioids (12 comparisons, } 17 \text { studies) }\end{array}$ & $\begin{array}{l}\text { Adverse effects for in- } \\
\text { fants }\end{array}$ & $\begin{array}{l}\text { No. of stud- } \\
\text { ies (no. } \\
\text { women) }\end{array}$ & Results \\
\hline IV fentanyl versus IV pethidine & Naloxone administration & $1(105)$ & $\begin{array}{l}\text { RR } 0.16,95 \% \mathrm{Cl} 0.02 \text { to } 1.28 \text {, no evidence of } \\
\text { a significant difference }\end{array}$ \\
\hline IV fentanyl versus IV pethidine & $\begin{array}{l}\text { Babies requiring resuscita- } \\
\text { tion/ventilatory support }\end{array}$ & $1(105)$ & $\begin{array}{l}\mathrm{RR} 1.03,95 \% \mathrm{Cl} 0.46 \text { to } 2.32 \text {, no evidence of } \\
\text { a significant difference }\end{array}$ \\
\hline IV fentanyl versus IV pethidine & $\begin{array}{l}\text { Neurobehavioural score } \\
\text { (1-2 hours after delivery) }\end{array}$ & $1(105)$ & $\begin{array}{l}\text { MD } 1.30,95 \% \mathrm{Cl} 0.15 \text { to } 2.45 \text {, no evidence } \\
\text { of a significant difference }\end{array}$ \\
\hline IV fentanyl versus IV pethidine & $\begin{array}{l}\text { Neurobehavioural score } \\
\text { (2-24 hours after delivery) }\end{array}$ & $1(105)$ & $\begin{array}{l}\mathrm{MD} 0.90,95 \% \mathrm{Cl}-0.42 \text { to } 2.22 \text {, no evidence } \\
\text { of a significant difference }\end{array}$ \\
\hline IV fentanyl versus IV butorphanol & $\begin{array}{l}\text { Babies requiring ventilato- } \\
\text { ry support }\end{array}$ & $1(100)$ & $\begin{array}{l}\text { RR } 11.00,95 \% \mathrm{Cl} 0.62 \text { to } 193.80 \text {, no evi- } \\
\text { dence of a significant difference }\end{array}$ \\
\hline IV fentanyl versus IV butorphanol & Naloxone required & $1(100)$ & $\begin{array}{l}\text { RR } 1.75,95 \% \mathrm{Cl} 0.81 \text { to } 3.80 \text {, no evidence of } \\
\text { a significant difference }\end{array}$ \\
\hline IV fentanyl versus IV butorphanol & $\begin{array}{l}\text { Neurobehavioural score } \\
\text { ( } 2-4 \text { hours after delivery) }\end{array}$ & $1(100)$ & $\begin{array}{l}\mathrm{MD} 0.00,95 \% \mathrm{Cl}-1.61 \text { to } 1.61 \text {, no evidence } \\
\text { of a significant difference }\end{array}$ \\
\hline IV fentanyl versus IV butorphanol & $\begin{array}{l}\text { Neurobehavioural score } \\
\text { (24-36 hours after delivery) }\end{array}$ & $1(100)$ & $\begin{array}{l}\mathrm{MD}-0.50,95 \% \mathrm{Cl}-1.62 \text { to } 0.62 \text {, no evidence } \\
\text { of a significant difference }\end{array}$ \\
\hline PCA remifentanil versus PCA pethidine & Naloxone administration & $2(56)$ & $\begin{array}{l}\text { RR } 0.30,95 \% \mathrm{Cl} 0.01 \text { to } 6.47 \text {, no evidence of } \\
\text { a significant difference }\end{array}$ \\
\hline PCA remifentanil versus PCA pethidine & $\begin{array}{l}\text { Neurobehavioural score } \\
\text { (15 minutes post delivery) }\end{array}$ & $1(56)$ & $\begin{array}{l}\mathrm{MD} 0.20,95 \% \mathrm{Cl}-0.93 \text { to } 1.33 \text {, no evidence } \\
\text { of a significant difference }\end{array}$ \\
\hline PCA remifentanil versus PCA pethidine & $\begin{array}{l}\text { Neurobehavioural score ( } 2 \\
\text { hours after delivery) }\end{array}$ & $1(59)$ & $\begin{array}{l}\mathrm{MD} 0.60,95 \% \mathrm{Cl}-0.66 \text { to } 1.86, \text { no evidence } \\
\text { of a significant difference }\end{array}$ \\
\hline PCA fentanyl versus PCA alftentanil & Naloxone required & $1(24)$ & $\begin{array}{l}\text { RR } 2.36,95 \% \mathrm{Cl} 0.53 \text { to } 10.55 \text {, no evidence } \\
\text { of a significant difference }\end{array}$ \\
\hline PCA fentanyl versus PCA pethidine & $\begin{array}{l}\text { Neurobehavioural score } \\
\text { (15 minutes post delivery) }\end{array}$ & $1(63)$ & $\begin{array}{l}\mathrm{MD}-0.90,95 \% \mathrm{Cl}-2.31 \text { to } 0.51 \text {, no evidence } \\
\text { of a significant difference }\end{array}$ \\
\hline PCA fentanyl versus PCA pethidine & $\begin{array}{l}\text { Neurobehavioural score ( } 2 \\
\text { hours post delivery) }\end{array}$ & $1(64)$ & $\begin{array}{l}\mathrm{MD}-0.50,95 \% \mathrm{Cl}-1.95 \text { to } 0.95 \text {, no evidence } \\
\text { of a significant difference }\end{array}$ \\
\hline $\begin{array}{l}\text { 2.2.2. IV opioids versus different IV } \\
\text { opioids (12 comparisons, } 17 \text { studies) }\end{array}$ & $\begin{array}{l}\text { Admission to special care } \\
\text { baby unit/neonatal in- } \\
\text { tensive care unit }\end{array}$ & $\begin{array}{l}\text { No. of stud- } \\
\text { ies (no. } \\
\text { women) }\end{array}$ & Results \\
\hline PCA remifentanil versus PCA pethidine & & $1(17)$ & $\begin{array}{l}\mathrm{RR} 0.30,95 \% \mathrm{Cl} 0.01 \text { to } 6.47 \text {, no evidence of } \\
\text { a significant difference }\end{array}$ \\
\hline
\end{tabular}


Table 24. (2.) Results by individual review - parenteral opioids - IV opioids versus different IV opioids/parenteral opioids versus different intervention (Continued)

$\begin{array}{lll}\begin{array}{l}\text { 2.2.2. IV opioids versus different IV } \\ \text { opioids (12 comparisons, } 17 \text { studies) }\end{array} & \begin{array}{l}\text { Apgar score less than } \\ \text { seven at five minutes }\end{array} & \begin{array}{l}\text { No. of stud- } \\ \text { ies (no. } \\ \text { women) }\end{array}\end{array}$

\begin{tabular}{lll} 
IV fentanyl versus IV pethidine & 1 (105) & $\begin{array}{l}\text { RR } 0.38,95 \% \mathrm{Cl} 0.02 \text { to } 9.12, \text { no evidence of } \\
\text { a significant difference }\end{array}$ \\
\hline IV butorphanol versus IV pethidine & $2(230)$ & $\begin{array}{l}\text { RR } 1.00,95 \% \mathrm{Cl} 0.06 \text { to } 15.77, \text { no evidence } \\
\text { of a significant difference }\end{array}$ \\
\hline IV fentanyl versus IV butorphanol & $1(100)$ & $\begin{array}{l}\text { RR } 1.20,95 \% \mathrm{Cl} 0.39 \text { to } 3.68, \text { no evidence of } \\
\text { a significant difference }\end{array}$ \\
\hline PCA remifentanil versus PCA pethidine & $1(17)$ & $\begin{array}{l}\text { RR } 0.13,95 \% \mathrm{Cl} 0.01 \text { to } 2.16, \text { no evidence of } \\
\text { a significant difference }\end{array}$ \\
\hline PCA nalbuphine versus PCA pethidine & $1(41)$ & $\begin{array}{l}\text { RR } 0.42,95 \% \mathrm{Cl} 0.02 \text { to } 9.76, \text { no evidence of } \\
\text { a significant difference }\end{array}$
\end{tabular}

$\begin{array}{ll}\begin{array}{l}\text { 2.2.2. IV opioids versus different IV } \\ \text { opioids (12 comparisons, } 17 \text { studies) }\end{array} & \begin{array}{l}\text { Poor infant outcomes at } \\ \text { follow-up }\end{array}\end{array}$

\section{Outcome not reported}

\begin{tabular}{l}
$\begin{array}{l}\text { 2.2.2. IV opioids versus different IV } \\
\text { opioids (12 comparisons, } 17 \text { studies) }\end{array}$ \\
\hline
\end{tabular}

\begin{tabular}{llll}
\hline $\begin{array}{l}\text { 2.3. Parenteral opioids versus differ- } \\
\text { ent interventions (3 comparisons, } 3 \\
\text { studies) }\end{array}$ & Pain intensity & $\begin{array}{l}\text { No. of stud- } \\
\text { ies (no. } \\
\text { women) }\end{array}$ & Results \\
\hline $\begin{array}{l}\text { Opioids (IM pethidine; IM tramadol) ver- } \\
\text { suS TENS }\end{array}$ & $\begin{array}{l}\text { Pain relief reported as } \\
\text { complete, excellent or } \\
\text { moderate at } 30 \text { minutes } \\
\text { after analgesia (1 study), } \\
\text { time point not reported in } \\
\text { the other study }\end{array}$ & $2(290)$ & $\begin{array}{l}\text { average RR } 1.15,95 \% \mathrm{Cl} 0.81 \text { to } 1.61(\mathrm{ran}- \\
\text { dom effects; heterogeneity: } \mathrm{I}^{2}=64 \%, \mathrm{~T}^{2}= \\
0.04, \mathrm{Chi} \text { test for heterogeneity } \mathrm{P}=0.10), \\
\text { no evidence of a significant difference }\end{array}$ \\
\hline
\end{tabular}

2.3. Parenteral opioids versus different interventions ( 3 comparisons, 3 studies)

Opioids (IV pethidine; IM pethidine) versus TENS

\subsection{Parenteral opioids versus differ-} ent interventions ( 3 comparisons, 3 studies)

\section{Satisfaction with pain re- $\quad$ No. of stud- Results lief ies (no. \\ women)}

Maternal satisfaction with 2 (104) analgesia measured post delivery (rated as good)
RR $1.23,95 \% \mathrm{Cl} 0.79$ to 1.92 , no evidence of a significant difference
Sense of control in Outcome not reported

labour
Outcome not reported

\subsection{Parenteral opioids versus differ- Satisfaction with child- ent interventions ( 3 comparisons, 3 birth experience studies)}

\subsection{Parenteral opioids versus differ- ent interventions ( 3 comparisons, 3 studies)}

\section{Effect on mother/baby interaction}


Table 24. (2.) Results by individual review - parenteral opioids - IV opioids versus different IV opioids/parenteral opioids versus different intervention (Continued)
2.3. Parenteral opioids versus differ-
Breastfeeding
Outcome not reported ent interventions ( 3 comparisons, 3 studies)

\begin{tabular}{|c|c|c|c|}
\hline $\begin{array}{l}\text { 2.3. Parenteral opioids versus differ- } \\
\text { ent interventions ( } 3 \text { comparisons, } 3 \\
\text { studies) }\end{array}$ & Assisted vaginal birth & $\begin{array}{l}\text { No. of stud- } \\
\text { ies (no. } \\
\text { women) }\end{array}$ & Results \\
\hline Opioids (IM tramadol) versus TENS & Assisted vaginal birth & $1(200)$ & $\begin{array}{l}\text { RR } 5.00,95 \% \mathrm{Cl} 0.24 \text { to } 102.85 \text {, no evidence } \\
\text { of a significant difference }\end{array}$ \\
\hline $\begin{array}{l}\text { 2.3. Parenteral opioids versus differ- } \\
\text { ent interventions ( } 3 \text { comparisons, } 3 \\
\text { studies) }\end{array}$ & Caesarean section & $\begin{array}{l}\text { No. of stud- } \\
\text { ies (no. } \\
\text { women) }\end{array}$ & Results \\
\hline Opioids (IM tramadol) versus TENS & Caesarean section & $1(200)$ & $\begin{array}{l}\text { Not estimable - no caesarean sections re- } \\
\text { ported in either the opioid or TENS group. }\end{array}$ \\
\hline $\begin{array}{l}\text { 2.3. Parenteral opioids versus differ- } \\
\text { ent interventions ( } 3 \text { comparisons, } 3 \\
\text { studies) }\end{array}$ & $\begin{array}{l}\text { Adverse effects for } \\
\text { women }\end{array}$ & $\begin{array}{l}\text { No. of stud- } \\
\text { ies (no. } \\
\text { women) }\end{array}$ & Results \\
\hline $\begin{array}{l}\text { Opioids (IM pethidine; IM tramadol) ver- } \\
\text { sus TENS }\end{array}$ & Drowsiness & $2(290)$ & $\begin{array}{l}\mathrm{RR} 8.96,95 \% \mathrm{Cl} 1.13 \text { to } 71.07 \text {, women in } \\
\text { the opioid group were more likely to report } \\
\text { drowsiness compared to those in the TENS } \\
\text { group }\end{array}$ \\
\hline $\begin{array}{l}\text { Opioids (IM pethidine; IM tramadol) ver- } \\
\text { sus TENS }\end{array}$ & Nausea and vomiting & $2(290)$ & $\begin{array}{l}\mathrm{RR} 14.06,95 \% \mathrm{Cl} 1.96 \text { to } 100.61 \text {, women in } \\
\text { the opioid group were more likely to report } \\
\text { nausea and vomiting compared to those in } \\
\text { the TENS group }\end{array}$ \\
\hline $\begin{array}{l}\text { 2.3. Parenteral opioids versus differ- } \\
\text { ent interventions ( } 3 \text { comparisons, } 3 \\
\text { studies) }\end{array}$ & $\begin{array}{l}\text { Adverse effects for in- } \\
\text { fants }\end{array}$ & $\begin{array}{l}\text { No. of stud- } \\
\text { ies (no. } \\
\text { women) }\end{array}$ & Results \\
\hline Opioids (IM tramadol) versus TENS & Fetal distress & $1(200)$ & $\begin{array}{l}\text { RR } 5.00,95 \% \mathrm{Cl} 0.24 \text { to } 102.85 \text {, no evidence } \\
\text { of a significant difference }\end{array}$ \\
\hline $\begin{array}{l}\text { 2.3. Parenteral opioids versus differ- } \\
\text { ent interventions ( } 3 \text { comparisons, } 3 \\
\text { studies) }\end{array}$ & $\begin{array}{l}\text { Admission to special care } \\
\text { baby unit }\end{array}$ & & Outcome not reported \\
\hline $\begin{array}{l}\text { 2.3. Parenteral opioids versus differ- } \\
\text { ent interventions ( } 3 \text { comparisons, } 3 \\
\text { studies) }\end{array}$ & $\begin{array}{l}\text { Apgar score less than } \\
\text { seven at five minutes }\end{array}$ & & Outcome not reported \\
\hline $\begin{array}{l}\text { 2.3. Parenteral opioids versus differ- } \\
\text { ent interventions ( } 3 \text { comparisons, } 3 \\
\text { studies) }\end{array}$ & $\begin{array}{l}\text { Poor infant outcomes at } \\
\text { follow-up }\end{array}$ & & Outcome not reported \\
\hline $\begin{array}{l}\text { 2.3. Parenteral opioids versus differ- } \\
\text { ent interventions ( } 3 \text { comparisons, } 3 \\
\text { studies) }\end{array}$ & Cost & & Outcome not reported \\
\hline
\end{tabular}


12 values reported in table only when random effects analysis has been carried out due to substantial heterogeneity indicated by an 12 value greater than $30 \%$

$\mathrm{Cl}$ : confidence interval; IV: intravenous; MD: mean difference; RR: risk ratio; SMD: standardised mean difference

Table 25. (3.) Results by individual review - non-opioid drugs

\section{Non-opioid drugs for pain management in labour, 18 studies, 2733 women (Othman 2012)}

\begin{tabular}{|c|c|c|c|}
\hline $\begin{array}{l}\text { 3.1. Non- } \\
\text { opioid } \\
\text { drugs ver- } \\
\text { sus place- } \\
\text { bo or no } \\
\text { treatment } \\
\text { (14 stud- } \\
\text { ies, } 2003 \\
\text { women) }\end{array}$ & Outcome & $\begin{array}{l}\text { No. of stud- } \\
\text { ies (no. } \\
\text { women) }\end{array}$ & Results \\
\hline
\end{tabular}

\begin{tabular}{|c|c|c|c|}
\hline Sedatives & Pain intensity $-10 \mathrm{~cm}$ VAS at one hour & $1(50)$ & $\begin{array}{l}\mathrm{MD}-22.00,95 \% \mathrm{Cl}-35.86 \text { to }-8.14 \text {, pain scores signif- } \\
\text { icantly lower in the non-opioid group }\end{array}$ \\
\hline $\begin{array}{l}\text { Anti-spas- } \\
\text { modics }\end{array}$ & $\begin{array}{l}\text { Pain intensity - women reporting severe pain } \\
\text { during first stage of labour }\end{array}$ & $1(84)$ & $\begin{array}{l}\text { RR } 0.88,95 \% \mathrm{Cl} 0.72 \text { to } 1.08 \text {, no evidence of a signif- } \\
\text { icant difference }\end{array}$ \\
\hline Sedatives & Satisfaction with pain relief & $2(204)$ & $\begin{array}{l}\mathrm{RR} 1.59,95 \% \mathrm{Cl} 1.15 \text { to } 2.21 \text {, women in the non-opi- } \\
\text { oid group were more likely to express satisfaction } \\
\text { with pain relief }\end{array}$ \\
\hline $\begin{array}{l}\text { Anti-hista- } \\
\text { mines }\end{array}$ & Satisfaction with pain relief & $1(223)$ & $\begin{array}{l}\mathrm{RR} 1.80,95 \% \mathrm{Cl} 1.16 \text { to } 2.79,1 \text { trial, women in the } \\
\text { non-opioid group were more likely to express satis- } \\
\text { faction with pain relief }\end{array}$ \\
\hline & Sense of control & & Outcome not reported \\
\hline Sedatives & Satisfaction with childbirth experience & $1(40)$ & $\begin{array}{l}\text { RR } 2.16,95 \% \mathrm{Cl} 1.34 \text { to } 3.47 \text {, women in the non-opi- } \\
\text { oid group were more likely to express satisfaction } \\
\text { with the childbirth experience }\end{array}$ \\
\hline & Effect (negative) on mother/baby interaction & & Outcome not reported \\
\hline Sedatives & $\begin{array}{l}\text { Breastfeeding (no definition of what was } \\
\text { meant by breastfeeding provided in the } \\
\text { study) }\end{array}$ & $1(198)$ & $\begin{array}{l}\text { RR } 0.65,95 \% \mathrm{Cl} 0.36 \text { to } 1.17 \text {, no evidence of a signif- } \\
\text { icant difference }\end{array}$ \\
\hline $\begin{array}{l}\text { Anti-spas- } \\
\text { modics }\end{array}$ & Assisted vaginal birth & $1(84)$ & $\begin{array}{l}\text { RR } 0.45,95 \% \mathrm{Cl} 0.09 \text { to } 2.35 \text {, no evidence of a signif- } \\
\text { icant difference }\end{array}$ \\
\hline Sedatives & Assisted vaginal birth & $3(417)$ & $\begin{array}{l}\text { RR } 0.93,95 \% \mathrm{Cl} 0.84 \text { to } 1.03 \text {, no evidence of a signif- } \\
\text { icant difference }\end{array}$ \\
\hline $\begin{array}{l}\text { Anti-hista- } \\
\text { mines }\end{array}$ & Assisted vaginal birth & $1(49)$ & $\begin{array}{l}\mathrm{RR} 3.12,95 \% \mathrm{Cl} 0.13 \text { to } 73.04 \text {, no evidence of a sig- } \\
\text { nificant difference }\end{array}$ \\
\hline Sedatives & Caesarean section & $2(203)$ & $\begin{array}{l}\text { RR } 3.62,95 \% \mathrm{Cl} 0.40 \text { to } 32.65 \text {, no evidence of a sig- } \\
\text { nificant difference }\end{array}$ \\
\hline
\end{tabular}


Table 25. (3.) Results by individual review - non-opioid drugs (Continued)

\begin{tabular}{|c|c|c|c|}
\hline $\begin{array}{l}\text { Anti-spas- } \\
\text { modics }\end{array}$ & $\begin{array}{l}\text { Adverse effects for women (Reported adverse } \\
\text { effects included nausea, vomiting, drowsi- } \\
\text { ness, tachycardia, headache, blurred vision, } \\
\text { dryness of the mouth, difficulty in micturition, } \\
\text { weakness of the legs, hypotension and atonic } \\
\text { postpartum haemorrhage) }\end{array}$ & $1(84)$ & $\begin{array}{l}\mathrm{RR} 7.27,95 \% \mathrm{Cl} 0.95 \text { to } 55.61 \text {, no evidence of a sig- } \\
\text { nificant difference }\end{array}$ \\
\hline
\end{tabular}

\begin{tabular}{lll}
\hline Sedatives & $\begin{array}{l}\text { Adverse effects for women (Reported adverse } \\
\text { effects included nausea, vomiting, drowsi- } \\
\text { ness, tachycardia, headache, blurred vision, } \\
\text { dryness of the mouth, difficulty in micturition, } \\
\text { weakness of the legs, hypotension and atonic } \\
\text { postpartum haemorrhage) }\end{array}$ & $\begin{array}{l}\text { RR } 1.26,95 \% \mathrm{Cl} 0.67 \text { to } 2.35, \text { no evidence of a signif- } \\
\text { icant difference }\end{array}$ \\
\end{tabular}

\begin{tabular}{llll}
\hline Sedatives & Adverse effects for infants & $2(387)$ & $\begin{array}{l}\text { RR 0.85, 95\% Cl 0.46 to 1.54, no evidence of a signif- } \\
\text { icant difference }\end{array}$ \\
\hline $\begin{array}{l}\text { Anti-hista- } \\
\text { mines }\end{array}$ & Adverse effects for infants & Outcome not reported \\
\hline
\end{tabular}

\begin{tabular}{|c|c|c|c|}
\hline & $\begin{array}{l}\text { Admission to special care baby unit/neonatal } \\
\text { intensive care unit }\end{array}$ & & Outcome not reported \\
\hline \multirow{3}{*}{$\begin{array}{l}\text { Seda- } \\
\text { tive-anal- } \\
\text { gesics }\end{array}$} & Apgar score less than seven at five minutes & $3(259)$ & $\begin{array}{l}\text { RR } 1.16,95 \% \mathrm{Cl} 0.55 \text { to } 2.45 \text {, no evidence of a signif- } \\
\text { icant difference }\end{array}$ \\
\hline & Poor infant outcomes at long-term follow-up & & Outcome not reported \\
\hline & Cost & & Outcome not reported \\
\hline \multirow{2}{*}{$\begin{array}{l}\text { 3.2. Non- } \\
\text { opioid drugs } \\
\text { versus dif- } \\
\text { ferent type } \\
\text { of non-opi- } \\
\text { oid drug ( } 2 \\
\text { studies, } 562 \\
\text { women) }\end{array}$} & Outcome & $\begin{array}{l}\text { No. of stud- } \\
\text { ies (no. } \\
\text { women) }\end{array}$ & Results \\
\hline & Pain intensity & & Outcome not reported \\
\hline $\begin{array}{l}\text { Sedatives } \\
\text { versus an- } \\
\text { ti-histamines }\end{array}$ & Satisfaction with pain relief & $1(157)$ & $\begin{array}{l}\mathrm{RR} 1.52,95 \% \mathrm{Cl} 1.06 \text { to } 2.17 \text {, women in the seda- } \\
\text { tive-analgesic group more likely to express satis- } \\
\text { faction with pain relief }\end{array}$ \\
\hline \multirow[t]{3}{*}{$\begin{array}{l}\text { Anti-hista- } \\
\text { mine versus } \\
\text { different an- } \\
\text { ti-histamine }\end{array}$} & Satisfaction with pain relief & $1(289)$ & $\begin{array}{l}\text { RR } 1.21,95 \% \mathrm{Cl} 1.02 \text { to } 1.43 \text {, women in the hydrox- } \\
\text { yzine + meperidine group were more likely to ex- } \\
\text { press satisfaction with pain relief when compared } \\
\text { to the promethazine + meperidine group }\end{array}$ \\
\hline & Sense of control in labour & & Outcome not reported \\
\hline & Satisfaction with childbirth experience & & Outcome not reported \\
\hline
\end{tabular}


Table 25. (3.) Results by individual review - non-opioid drugs (Continued)

Effect (negative) on mother/baby interaction Outcome not reported

\begin{tabular}{llll}
\hline & Breastfeeding & Outcome not reported \\
\hline $\begin{array}{l}\text { Anti-hista- } \\
\text { mine versus } \\
\begin{array}{l}\text { different an- } \\
\text { ti-histamine }\end{array}\end{array}$ & Assisted vaginal birth & $1(289)$ & $\begin{array}{l}\text { RR 0.82, 95\% Cl 0.64 to 1.03, no evidence of a signif- } \\
\text { icant difference }\end{array}$ \\
\hline & Caesarean section & Outcome not reported \\
\hline $\begin{array}{l}\text { Sedatives } \\
\text { versus an- } \\
\text { ti-histamines }\end{array}$ & Adverse effects for women & Outcome not reported \\
\hline
\end{tabular}

\begin{tabular}{|c|c|c|c|}
\hline & $\begin{array}{l}\text { Admission to special care baby unit/neonatal } \\
\text { intensive care }\end{array}$ & & Outcome not reported \\
\hline \multirow[t]{3}{*}{$\begin{array}{l}\text { Anti-hista- } \\
\text { mine versus } \\
\text { different an- } \\
\text { ti-histamine }\end{array}$} & Apgar score less than seven at five minutes & $1(289)$ & $\begin{array}{l}\mathrm{RR} 1.85,95 \% \mathrm{Cl} 0.17 \text { to } 20.21 \text {, no evidence of a sig- } \\
\text { nificant difference }\end{array}$ \\
\hline & Poor infant outcomes at long-term follow-up & & Outcome not reported \\
\hline & Cost & & Outcome not reported \\
\hline \multirow[t]{2}{*}{$\begin{array}{l}\text { 3.2. Non- } \\
\text { opioid drug } \\
\text { versus same } \\
\text { non-opi- } \\
\text { oid drug } \\
\text { of a differ- } \\
\text { ent dose (1 } \\
\text { study, } 28 \\
\text { women) }\end{array}$} & Outcome & $\begin{array}{l}\text { No. of stud- } \\
\text { ies (no. } \\
\text { women) }\end{array}$ & Results \\
\hline & Pain intensity & & Outcome not reported \\
\hline \multirow[t]{6}{*}{ Sedatives } & Satisfaction with pain relief & $1(19)$ & $\begin{array}{l}\text { RR } 0.22,95 \% \mathrm{Cl} 0.04 \text { to } 1.33 \text {, no evidence of a signif- } \\
\text { icant difference }\end{array}$ \\
\hline & Sense of control in labour & & Outcome not reported \\
\hline & Satisfaction with childbirth experience & & Outcome not reported \\
\hline & Effect (negative) on mother/baby interaction & & Outcome not reported \\
\hline & Breastfeeding & & Outcome not reported \\
\hline & Assisted vaginal birth & & Outcome not reported \\
\hline Sedatives & Caesarean section & $1(19)$ & $\begin{array}{l}\text { RR } 6.00,95 \% \mathrm{Cl} 0.28 \text { to } 129.16 \text {, no evidence of a sig- } \\
\text { nificant difference }\end{array}$ \\
\hline
\end{tabular}


Table 25. (3.) Results by individual review - non-opioid drugs (Continued)

oids -3 arm

trial)

\begin{tabular}{ll}
\hline Adverse effects for infants & Outcome not reported \\
\hline $\begin{array}{l}\text { Admission to special care baby unit/neonatal } \\
\text { intensive care unit }\end{array}$ & Outcome not reported \\
\hline Apgar score less than seven at five minutes & Outcome not reported \\
\hline Poor infant outcomes at long-term follow-up & Outcome not reported \\
\hline Cost & Outcome not reported \\
\hline
\end{tabular}

12 values reported in table only when random effects analysis has been carried out due to substantial heterogeneity indicated by an 12 value greater than $30 \%$

$\mathrm{Cl}$ : confidence interval; MD: mean difference; RR: risk ratio

Table 26. (4.) Results by individual review - local anaesthetic nerve block

4. Local anaesthetic nerve block for pain management in labour, 12 studies, 1549 women (Novikova 2012)

\begin{tabular}{lll}
\hline $\begin{array}{l}\text { 4.1. Local anaesthet- } \\
\text { ic nerve block versus }\end{array}$ & Outcome & $\begin{array}{l}\text { No. of stud- } \\
\text { ies (no. } \\
\text { placebo (1 study, } 200\end{array}$ \\
women) & women)
\end{tabular}

women)

\begin{tabular}{|c|c|c|c|}
\hline & Pain intensity & & Outcome not reported \\
\hline \multirow[t]{10}{*}{$\begin{array}{l}\text { PCB with } 2 \% \text { lidocaine } \\
\text { versus PCB with dis- } \\
\text { tilled water }\end{array}$} & $\begin{array}{l}\text { Satisfaction with pain relief (degree of pain re- } \\
\text { lief rated as excellent/complete) }\end{array}$ & $1(198)$ & $\begin{array}{l}\mathrm{RR} 32.31,95 \% \mathrm{Cl} 10.60 \text { to } 98.54 \text {, more } \\
\text { women in the lidocaine group were } \\
\text { satisfied with pain relief }\end{array}$ \\
\hline & Sense of control in labour & & Outcome not reported \\
\hline & Satisfaction with childbirth experience & & Outcome not reported \\
\hline & Effect on mother/baby interaction & & Outcome not reported \\
\hline & Breastfeeding & & Outcome not reported \\
\hline & Assisted vaginal birth & & Outcome not reported \\
\hline & Caesarean section & & Outcome not reported \\
\hline & $\begin{array}{l}\text { Adverse effects for women and infants (mother } \\
\text { - giddiness, sweating, tingling of lower limbs; } \\
\text { baby- bradycardia) }\end{array}$ & $1(200)$ & $\begin{array}{l}\text { RR } 29.00,95 \% \mathrm{Cl} 1.75 \text { to } 479.61 \text {, signif- } \\
\text { icantly more side-effects in mothers } \\
\text { and babies in the lidocaine group }\end{array}$ \\
\hline & $\begin{array}{l}\text { Admission to special care baby unit/neonatal } \\
\text { intensive care unit }\end{array}$ & & Outcome not reported \\
\hline & Apgar score less than seven at five minutes & & Outcome not reported \\
\hline
\end{tabular}


Table 26. (4.) Results by individual review - local anaesthetic nerve block (Continued)

Poor infant outcomes at long-term follow-up

Cost

\section{Outcome}

4.2. Local anaesthetic nerve block versus opioids (2 studies, 129 women)
Outcome not reported

Outcome not reported

\begin{tabular}{|c|c|c|c|}
\hline & Pain intensity & & Outcome not reported \\
\hline \multirow[t]{5}{*}{$\begin{array}{l}\text { PCB versus intramuscu- } \\
\text { lar pethidine }\end{array}$} & $\begin{array}{l}\text { Satisfaction with pain relief (pain relief dur- } \\
\text { ing } 1^{\text {st }} \text { hour after administration of analgesia } \\
\text { "complete" or "acceptable" }\end{array}$ & $1(109)$ & $\begin{array}{l}\mathrm{RR} 2.52,95 \% \mathrm{Cl} 1.65 \text { to } 3.83 \text {, more } \\
\text { women in the } \mathrm{PCB} \text { group were satisfied } \\
\text { with pain relief }\end{array}$ \\
\hline & Sense of control in labour & & Outcome not reported \\
\hline & Satisfaction with childbirth experience & & Outcome not reported \\
\hline & Effect on mother/baby interaction & & Outcome not reported \\
\hline & Breastfeeding & & Outcome not reported \\
\hline $\begin{array}{l}\text { PCB versus intramus- } \\
\text { cular pethidine or pa- } \\
\text { tient controlled analge- } \\
\text { sia with fentanyl }\end{array}$ & Assisted vaginal birth & $2(129)$ & $\begin{array}{l}\text { RR } 1.02,95 \% \mathrm{Cl} 0.56 \text { to } 1.87 \text {, no evi- } \\
\text { dence of a significant difference be- } \\
\text { tween groups }\end{array}$ \\
\hline $\begin{array}{l}\text { PCB versus intramus- } \\
\text { cular pethidine or pa- } \\
\text { tient controlled analge- } \\
\text { sia with fentanyl }\end{array}$ & Caesarean section & $2(129)$ & $\begin{array}{l}\text { RR } 0.23,95 \% \mathrm{Cl} 0.03 \text { to } 1.87 \text {, no evi- } \\
\text { dence of a significant difference be- } \\
\text { tween groups }\end{array}$ \\
\hline
\end{tabular}

\begin{tabular}{|c|c|c|c|}
\hline & Adverse effects for women (vomiting) & & Outcome not reported \\
\hline & Adverse effects for infants & & Outcome not reported \\
\hline & $\begin{array}{l}\text { Admission to special care baby unit/neonatal } \\
\text { intensive care unit }\end{array}$ & & Outcome not reported \\
\hline $\begin{array}{l}\text { PCB versus intramus- } \\
\text { cular pethidine or pa- } \\
\text { tient controlled analge- } \\
\text { sia with fentanyl }\end{array}$ & Apgar score less than seven at five minutes & $2(122)$ & $\begin{array}{l}\text { Not estimable - None of the babies had } \\
\text { Apgar score less than seven at five min- } \\
\text { utes }\end{array}$ \\
\hline
\end{tabular}

\begin{tabular}{ll}
\hline Poor infant outcomes at long-term follow-up & Outcome not reported \\
\hline Cost & Outcome not reported
\end{tabular}

\subsection{Local anaesthet- ic nerve block versus non-opioid (1 study, 100 women)}

\section{Outcome}

\author{
No. of stud- Results \\ ies (no. \\ women)
}

\author{
Results \\ women)
}


Table 26. (4.) Results by individual review - local anaesthetic nerve block (Continued)

\begin{tabular}{|c|c|c|c|}
\hline & Pain intensity & & Outcome not reported \\
\hline \multirow[t]{6}{*}{$\begin{array}{l}\text { PCB versus intramuscu- } \\
\text { lar promethazine }\end{array}$} & $\begin{array}{l}\text { Satisfaction with pain relief (excellent or com- } \\
\text { plete relief) }\end{array}$ & $1(100)$ & $\begin{array}{l}\text { RR } 1.11,95 \% \mathrm{Cl} 0.67 \text { to } 1.84 \text {, no evi- } \\
\text { dence of a significant difference be- } \\
\text { tween groups }\end{array}$ \\
\hline & Sense of control in labour & & Outcome not reported \\
\hline & Satisfaction with childbirth experience & & Outcome not reported \\
\hline & Effect on mother/baby interaction & & Outcome not reported \\
\hline & Breastfeeding & & Outcome not reported \\
\hline & Assisted vaginal birth & & Outcome not reported \\
\hline $\begin{array}{l}\text { PCB versus intramuscu- } \\
\text { lar promethazine }\end{array}$ & Caesarean section & $1(100)$ & $\begin{array}{l}\text { RR } 2.00,95 \% \mathrm{Cl} 0.19 \text { to } 21.36 \text {, no ev- } \\
\text { idence of a significant difference be- } \\
\text { tween groups }\end{array}$ \\
\hline
\end{tabular}

Adverse effects for women

Outcome not reported

Adverse effects for infants

Outcome not reported

Admission to special care baby unit/neonatal intensive care unit

Outcome not reported

Apgar score less than seven at five minutes $\quad 1(100)$

PCB versus intramuscu- Apgar score less than seven at five minutes

$1(100)$

Not estimable - None of the babies had Apgar score less than seven at five minutes

Poor infant outcomes at long-term follow-up

Outcome not reported

Cost

Outcome not reported

\subsection{Local anaesthetic nerve block versus dif- ferent dose/agent or timing of anaesthetic nerve block ( 8 studies, 1120 women) \\ Outcome

e

\author{
No. of stud- Results \\ ies (no. \\ women)
}

\begin{tabular}{|c|c|c|c|}
\hline & Pain intensity & & Outcome not reported \\
\hline $\begin{array}{l}\text { PCB } 1 \% \text { lidocaine ver- } \\
\text { sus } 2 \% \text { chloroprocaine }\end{array}$ & $\begin{array}{l}\text { Satisfaction with pain relief - (proportion with } \\
\text { unsatisfactory pain relief) }\end{array}$ & $1(60)$ & $\begin{array}{l}\mathrm{RR} 2.81,95 \% \mathrm{Cl} 0.31 \text { to } 25.48 \text {, no ev- } \\
\text { idence of a significant difference be- } \\
\text { tween groups }\end{array}$ \\
\hline \multirow[t]{3}{*}{$\begin{array}{l}\text { PCB } 0.25 \% \text { bupivacaine } \\
\text { versus } 2 \% \text { chloropro- } \\
\text { caine or } 1 \% \text { carbacaine }\end{array}$} & $\begin{array}{l}\text { Satisfaction with pain relief during the second } \\
\text { stage of labour (proportion with good or excel- } \\
\text { lent pain relief) }\end{array}$ & $2(332)$ & $\begin{array}{l}\mathrm{RR} 0.93,95 \% \mathrm{Cl} 0.85 \text { to } 1.00 \text {, no evi- } \\
\text { dence of a significant difference be- } \\
\text { tween groups }\end{array}$ \\
\hline & Sense of control in labour & & Outcome not reported \\
\hline & Satisfaction with childbirth experience & & Outcome not reported \\
\hline
\end{tabular}


Table 26. (4.) Results by individual review - local anaesthetic nerve block (Continued)

\begin{tabular}{|c|c|c|c|}
\hline & \multicolumn{2}{|l|}{ Effect on mother/baby interaction } & \multirow{2}{*}{$\begin{array}{l}\text { Outcome not reported } \\
\text { Outcome not reported }\end{array}$} \\
\hline & Breastfeeding & & \\
\hline $\begin{array}{l}\text { PCB } 1 \% \text { lidocaine ver- } \\
\text { sus } 2 \% \text { chloroprocaine }\end{array}$ & Assisted vaginal birth & $1(60)$ & $\begin{array}{l}\text { RR } 0.57,95 \% \mathrm{Cl} 0.29 \text { to } 1.14 \text {, no evi- } \\
\text { dence of a significant difference be- } \\
\text { tween groups }\end{array}$ \\
\hline $\begin{array}{l}\text { PCB } 1 \% \text { lidocaine ver- } \\
\text { sus } 2 \% \text { chloroprocaine }\end{array}$ & Caesarean section & $1(58)$ & $\begin{array}{l}\mathrm{RR} 1.00,95 \% \mathrm{Cl} 0.15 \text { to } 6.63 \text {, no evi- } \\
\text { dence of a significant difference be- } \\
\text { tween groups }\end{array}$ \\
\hline \multirow[t]{3}{*}{$\begin{array}{l}\text { PCB } 0.25 \% \text { bupivacaine } \\
\text { versus } 1 \% \text { carbacaine }\end{array}$} & $\begin{array}{l}\text { Adverse effects for women (slight dizziness af- } \\
\text { ter injection) }\end{array}$ & $1(285)$ & $\begin{array}{l}\mathrm{RR} 0.30,95 \% \mathrm{Cl} 0.03 \text { to } 2.89 \text {, no evi- } \\
\text { dence of a significant difference be- } \\
\text { tween groups }\end{array}$ \\
\hline & Adverse effects for infants & & Outcome not reported \\
\hline & $\begin{array}{l}\text { Admission to special care baby unit/neonatal } \\
\text { intensive care }\end{array}$ & & Outcome not reported \\
\hline \multirow[t]{3}{*}{$\begin{array}{l}\text { PCB } 0.25 \% \text { bupivacaine } \\
\text { versus } 2 \% \text { chloropro- } \\
\text { caine }\end{array}$} & Apgar score less than seven at five minutes & $1(47)$ & $\begin{array}{l}\mathrm{RR} 0.32,95 \% \mathrm{Cl} 0.01 \text { to } 7.48 \text {, no evi- } \\
\text { dence of a significant difference be- } \\
\text { tween groups }\end{array}$ \\
\hline & Poor infant outcomes at long-term follow-up & & Outcome not reported \\
\hline & Cost & & Outcome not reported \\
\hline
\end{tabular}

Cl: confidence interval; RR: risk ratio

Table 27. (5.) Results by individual review - epidural

5. Epidural versus non-epidural or no analgesia in labour, 38 studies, 9658 women (Anim-Somuah 2011)

\begin{tabular}{|c|c|c|c|}
\hline $\begin{array}{l}\text { 5.1. Epidur- } \\
\text { al versus } \\
\text { non-epidur- } \\
\text { al/no anal- } \\
\text { gesia }\end{array}$ & Outcome & $\begin{array}{l}\text { No. of stud- } \\
\text { ies (no. } \\
\text { women) }\end{array}$ & Results \\
\hline
\end{tabular}

\begin{tabular}{|c|c|c|}
\hline $\begin{array}{l}\text { Pain intensity (during whole of } \\
\text { labour), using visual analogue score } \\
\text { (VAS) } 0 \text { to } 10 \text {, where } 0 \text { represents no } \\
\text { pain and } 10 \text { worst pain }\end{array}$ & $\begin{array}{l}3 \text { (1166 } \\
\text { women) }\end{array}$ & $\begin{array}{l}\text { Average mean difference (MD) }-3.36,95 \% \mathrm{Cl}-5.41 \text { to }-1.31 \\
\text { (random effects: heterogeneity: } \mathrm{I}^{2}=98 \%, \mathrm{Tau}^{2}=3.14, \mathrm{Chi}^{2} \\
\text { test for heterogeneity } \mathrm{P}<0.00001 \text { ), a significant reduction in } \\
\text { pain intensity for women receiving epidural }\end{array}$ \\
\hline $\begin{array}{l}\text { Pain intensity (in the first stage of } \\
\text { labour) VAS }\end{array}$ & $\begin{array}{l}4(589 \\
\text { women) }\end{array}$ & $\begin{array}{l}\text { Average mean difference (MD) }-16.35,95 \% \mathrm{Cl}-25.11 \text { to }-7.58 \\
\text { (random effects: heterogeneity: } \mathrm{I}^{2}=87 \%, \mathrm{Tau}^{2}=65.03, \mathrm{Chi}^{2} \\
\text { test for heterogeneity } \mathrm{P}<0.0001 \text { ), a significant reduction in } \\
\text { pain intensity for women receiving epidural }\end{array}$ \\
\hline $\begin{array}{l}\text { Pain intensity (in the second stage } \\
\text { of labour) VAS }\end{array}$ & $\begin{array}{l}3(559 \\
\text { women) }\end{array}$ & $\begin{array}{l}\text { Average mean difference (MD) }-25.29,95 \% \mathrm{Cl}-40.48 \text { to }-10.11 \\
\text { (random effects: heterogeneity: } \mathrm{I}^{2}=96 \%, \mathrm{Tau}^{2}=162.74, \mathrm{Chi}^{2} \\
\text { test for heterogeneity } \mathrm{P}<0.00001 \text { ), a significant reduction in } \\
\text { pain intensity for women receiving epidural }\end{array}$ \\
\hline
\end{tabular}


Table 27. (5.) Results by individual review - epidural (Continued)

\begin{tabular}{|c|c|c|}
\hline $\begin{array}{l}\text { Maternal satisfaction with pain re- } \\
\text { lief - dichotomous data: the pro- } \\
\text { portion of women rating their sat- } \\
\text { isfaction with analgesia as excel- } \\
\text { lent, very good, good after delivery } \\
\text { in each group }\end{array}$ & $\begin{array}{l}7(2929 \\
\text { women) }\end{array}$ & $\begin{array}{l}\text { Average RR } 1.31,95 \% \mathrm{Cl} 0.84 \text { to } 2.05 \text { (random effects: hetero- } \\
\text { geneity: }\left.\right|^{2}=100 \%, \mathrm{Tau}^{2}=0.36, \mathrm{Chi}^{2} \text { test for heterogeneity } \mathrm{P} \\
<0.00001 \text { ), no evidence of a significant difference }\end{array}$ \\
\hline $\begin{array}{l}\text { Maternal satisfaction with pain re- } \\
\text { lief - continuous data }\end{array}$ & $\begin{array}{l}2(272 \\
\text { women) }\end{array}$ & $\begin{array}{l}\text { Average standardised mean difference (SMD) } 0.10,95 \% \mathrm{Cl} \\
-0.49 \text { to } 0.70 \text { (random effects: heterogeneity: }\left.\right|^{2}=62 \%, \mathrm{Tau}^{2} \\
\left.=0.12, \mathrm{Ch}{ }^{2} \text { test for heterogeneity } \mathrm{P}=0.10\right) \text {, no evidence of a } \\
\text { significant difference }\end{array}$ \\
\hline $\begin{array}{l}\text { Sense of control in labour (feelings } \\
\text { of poor control) }\end{array}$ & $\begin{array}{l}1(344 \\
\text { women) }\end{array}$ & $\begin{array}{l}\mathrm{RR} 1.17,95 \% \mathrm{Cl} 0.62 \text { to } 2.21 \text {, no evidence of a significant dif- } \\
\text { ference }\end{array}$ \\
\hline $\begin{array}{l}\text { Satisfaction with childbirth experi- } \\
\text { ence }\end{array}$ & $\begin{array}{l}1(332 \\
\text { women) }\end{array}$ & $\begin{array}{l}\text { RR } 0.95,95 \% \mathrm{Cl} 0.87 \text { to } 1.03 \text {, no evidence of a significant dif- } \\
\text { ference }\end{array}$ \\
\hline $\begin{array}{l}\text { Effect (negative) on mother/baby in- } \\
\text { teraction }\end{array}$ & & Outcome not reported \\
\hline Breastfeeding & & Outcome not reported \\
\hline Assisted vaginal birth & $\begin{array}{l}23(7935 \\
\text { women) }\end{array}$ & $\begin{array}{l}\text { RR } 1.42,95 \% \mathrm{Cl} 1.28 \text { to } 1.57 \text {, significantly more women in the } \\
\text { epidural group had assisted vaginal birth }\end{array}$ \\
\hline Caesarean section & $\begin{array}{l}27(8417 \\
\text { women) }\end{array}$ & $\begin{array}{l}\text { RR } 1.10,95 \% \mathrm{Cl} 0.97 \text { to } 1.25 \text {, no evidence of a significant dif- } \\
\text { ference }\end{array}$ \\
\hline Caesarean section for fetal distress & $\begin{array}{l}11(4816 \\
\text { women) }\end{array}$ & $\begin{array}{l}\mathrm{RR} 1.43,95 \% \mathrm{Cl} 1.03 \text { to } 1.97,11 \text { trials, an increased risk of } \\
\text { caesarean section for fetal distress }\end{array}$ \\
\hline $\begin{array}{l}\text { Adverse effects for women (long- } \\
\text { term backache) }\end{array}$ & $\begin{array}{l}3(1806 \\
\text { women) }\end{array}$ & $\begin{array}{l}\text { RR } 0.96,95 \% \mathrm{Cl} 0.86 \text { to } 1.07 \text {, no evidence of a significant dif- } \\
\text { ference }\end{array}$ \\
\hline $\begin{array}{l}\text { Adverse effects for women (mater- } \\
\text { nal hypotension) }\end{array}$ & $\begin{array}{l}8(2789 \\
\text { women })\end{array}$ & $\begin{array}{l}\text { RR } 18.23,95 \% \mathrm{Cl} 5.09 \text { to } 65.35, \text { (random effects: heterogene- } \\
\text { ity: }{ }^{2}=47 \%, \mathrm{Tau}^{2}=1.57, \mathrm{Chi}^{2} \text { test for heterogeneity } \mathrm{P}=0.07 \text { ), } \\
\text { significantly greater risk of hypotension in women in the } \\
\text { epidural group }\end{array}$ \\
\hline $\begin{array}{l}\text { Adverse effects for women (postna- } \\
\text { tal depression) }\end{array}$ & $\begin{array}{l}1(313 \\
\text { women) }\end{array}$ & $\begin{array}{l}\text { RR } 0.63,95 \% \mathrm{Cl} 0.38 \text { to } 1.05 \text {, no evidence of a significant dif- } \\
\text { ference }\end{array}$ \\
\hline $\begin{array}{l}\text { Adverse effects for women (motor } \\
\text { blockade) }\end{array}$ & $\begin{array}{l}3(322 \\
\text { women) }\end{array}$ & $\begin{array}{l}\text { RR } 31.67,95 \% \mathrm{Cl} 4.33 \text { to } 231.51 \text {, significantly greater risk of } \\
\text { motor blockade in women in the epidural group }\end{array}$ \\
\hline $\begin{array}{l}\text { Adverse effects for women } \\
\text { (headache) }\end{array}$ & $\begin{array}{l}3 \text { (1198 } \\
\text { women) }\end{array}$ & $\begin{array}{l}\text { RR } 0.96,95 \% \mathrm{Cl} 0.81 \text { to } 1.15 \text {, no evidence of a significant dif- } \\
\text { ference }\end{array}$ \\
\hline $\begin{array}{l}\text { Adverse effects for women (nausea } \\
\text { and vomiting) }\end{array}$ & $\begin{array}{l}12(3187 \\
\text { women) }\end{array}$ & $\begin{array}{l}\text { Average RR } 0.95,95 \% \mathrm{Cl} 0.72 \text { to } 1.27 \text {, random effects: hetero- } \\
\text { geneity: }\left.\right|^{2}=49 \%, \mathrm{Tau}^{2}=0.09, \mathrm{Chi} 2 \text { test for heterogeneity } \mathrm{P}= \\
0.03) \text {, no evidence of a significant difference }\end{array}$ \\
\hline Adverse effects for women (itching) & $\begin{array}{l}3(230 \\
\text { women) }\end{array}$ & $\begin{array}{l}\text { RR } 1.46,95 \% \mathrm{Cl} 0.51 \text { to } 4.16 \text {, no evidence of a significant dif- } \\
\text { ference }\end{array}$ \\
\hline
\end{tabular}


Table 27. (5.) Results by individual review - epidural (Continued)

\begin{tabular}{|c|c|c|}
\hline Adverse effects for women (fever) & $\begin{array}{l}6(2741 \\
\text { women })\end{array}$ & $\begin{array}{l}\mathrm{RR} 3.34,95 \% \mathrm{Cl} 2.63 \text { to } 4.23 \text {, significantly greater risk of fever } \\
\text { in women in the epidural group }\end{array}$ \\
\hline $\begin{array}{l}\text { Adverse effects for women (shiver- } \\
\text { ing) }\end{array}$ & $\begin{array}{l}1 \text { ( } 20 \\
\text { women) }\end{array}$ & $\begin{array}{l}\text { RR } 5.00,95 \% \mathrm{Cl} 0.27 \text { to } 92.62 \text {, no evidence of a significant dif- } \\
\text { ference }\end{array}$ \\
\hline $\begin{array}{l}\text { Adverse effects for women (drowsi- } \\
\text { ness) }\end{array}$ & $\begin{array}{l}4(641 \\
\text { women) }\end{array}$ & $\begin{array}{l}\text { Average RR } 0.55,95 \% \mathrm{Cl} 0.07 \text { to } 4.26 \text {, random effects: hetero- } \\
\text { geneity } \mathrm{I}^{2}=94 \%, \mathrm{Tau}^{2}=3.47, \mathrm{Chi}^{2} \text { test for heterogeneity } \mathrm{P}< \\
0.00001) \text {, no evidence of a significant difference }\end{array}$ \\
\hline $\begin{array}{l}\text { Adverse effects for women (urinary } \\
\text { retention) }\end{array}$ & $\begin{array}{l}3(283 \\
\text { women) }\end{array}$ & $\begin{array}{l}\mathrm{RR} 17.05,95 \% \mathrm{Cl} 4.82 \text { to } 60.39 \text {, significantly greater risk of } \\
\text { urinary retention in women in the epidural group }\end{array}$ \\
\hline $\begin{array}{l}\text { Adverse effects for women } \\
\text { (catheterization during labour) }\end{array}$ & $\begin{array}{l}2 \text { (1103 } \\
\text { women) }\end{array}$ & $\begin{array}{l}\text { RR } 1.81,95 \% \mathrm{Cl} 0.44 \text { to } 7.46 \text {, no evidence of a significant dif- } \\
\text { ference }\end{array}$ \\
\hline $\begin{array}{l}\text { Adverse effects for women (malposi- } \\
\text { tion) }\end{array}$ & $\begin{array}{l}4(673 \\
\text { women) }\end{array}$ & $\begin{array}{l}\text { RR } 1.40,95 \% \mathrm{Cl} 0.98 \text { to } 1.99 \text {, no evidence of a significant dif- } \\
\text { ference }\end{array}$ \\
\hline $\begin{array}{l}\text { Adverse effects for women (surgical } \\
\text { amniotomy) }\end{array}$ & $\begin{array}{l}2(211 \\
\text { women) }\end{array}$ & $\begin{array}{l}\text { Average RR } 1.03,95 \% \mathrm{Cl} 0.74 \text { to } 1.43 \text {, random effects: hetero- } \\
\text { geneity } \mathrm{I}^{2}=81 \%, \mathrm{Tau}^{2}=0.05, \mathrm{Chi}^{2} \text { test for heterogeneity } \mathrm{P}= \\
0.02 \text {, no evidence of a significant difference }\end{array}$ \\
\hline $\begin{array}{l}\text { Adverse effects for infants (acidosis } \\
\mathrm{pH} \text { less than 7.2) }\end{array}$ & $\begin{array}{l}7(3643 \\
\text { women) }\end{array}$ & $\begin{array}{l}\text { RR } 0.80,95 \% \mathrm{Cl} 0.68 \text { to } 0.94 \text {, neonates of women who had } \\
\text { epidural had a significantly lower risk of acidosis }\end{array}$ \\
\hline $\begin{array}{l}\text { Adverse effects for infants (acidosis } \\
\mathrm{pH} \text { less than } 7.15 \text { ) }\end{array}$ & $\begin{array}{l}2(382 \\
\text { women) }\end{array}$ & $\begin{array}{l}\text { RR } 0.95,95 \% \mathrm{Cl} 0.50 \text { to } 1.79 \text {, no evidence of a significant dif- } \\
\text { ference }\end{array}$ \\
\hline $\begin{array}{l}\text { Adverse effects for infants (naloxone } \\
\text { administration) }\end{array}$ & $\begin{array}{l}10(2645 \\
\text { women) }\end{array}$ & $\begin{array}{l}\mathrm{RR} 0.15,95 \% \mathrm{Cl} 0.10 \text { to } 0.23 \text {, neonates of women who had } \\
\text { epidural had a significantly lower risk of requiring naloxone }\end{array}$ \\
\hline $\begin{array}{l}\text { Adverse effects for infants (meconi- } \\
\text { um staining of liquor) }\end{array}$ & $\begin{array}{l}5(2295 \\
\text { women) }\end{array}$ & $\begin{array}{l}\text { RR } 1.01,95 \% \mathrm{Cl} 0.84 \text { to } 1.21 \text {, no evidence of a significant dif- } \\
\text { ference }\end{array}$ \\
\hline $\begin{array}{l}\text { Admission to special care baby unit/ } \\
\text { neonatal intensive care unit }\end{array}$ & $\begin{array}{l}7(3125 \\
\text { women) }\end{array}$ & $\begin{array}{l}\mathrm{RR} 1.19,95 \% \mathrm{Cl} 0.94 \text { to } 1.50 \text {, no evidence of a significant dif- } \\
\text { ference }\end{array}$ \\
\hline $\begin{array}{l}\text { Apgar score less than seven at five } \\
\text { minutes }\end{array}$ & $\begin{array}{l}18(6898 \\
\text { women) }\end{array}$ & $\begin{array}{l}\text { RR } 0.80,95 \% \mathrm{Cl} 0.54 \text { to } 1.20 \text {, no evidence of a significant dif- } \\
\text { ference }\end{array}$ \\
\hline $\begin{array}{l}\text { Poor infant outcomes at long-term } \\
\text { follow-up }\end{array}$ & & Outcome not reported \\
\hline Cost & & Outcome not reported \\
\hline
\end{tabular}

$\mathrm{I}^{2}$ values reported in table only when random effects analysis has been carried out due to substantial heterogeneity indicated by an 12 value greater than $30 \%$

$\mathrm{Cl}$ : confidence interval; MD: mean difference; RR: risk ratio

Table 28. (6.) Results by individual review - combined spinal epidural

6. Combined spinal-epidural versus epidural in labour, 27 studies, 3303 women (Simmons 2012) 
Table 28. (6.) Results by individual review - combined spinal epidural (Continued)

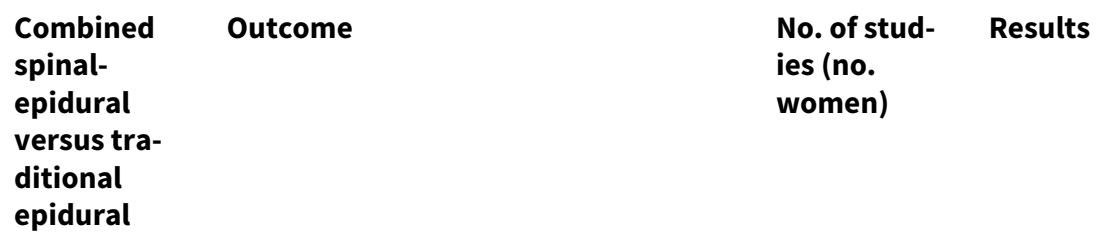

Pain intensity - time from first injection to effective analgesia in minutes
$2(129)$

MD -2.87, $95 \% \mathrm{Cl}-5.07$ to -0.67, CSE had a faster onset time of effective pain relief from time of injection (approximately 3 minutes)

\begin{tabular}{|c|c|c|}
\hline \multicolumn{2}{|l|}{ Satisfaction with pain relief } & Outcome not reported \\
\hline \multicolumn{2}{|l|}{ Sense of control in labour } & Outcome not reported \\
\hline \multicolumn{2}{|l|}{ Satisfaction with childbirth experience } & Outcome not reported \\
\hline \multicolumn{2}{|l|}{ Effect on mother/baby interaction } & Outcome not reported \\
\hline \multicolumn{2}{|l|}{ Breastfeeding } & Outcome not reported \\
\hline Assisted vaginal birth & $6(1015)$ & $\begin{array}{l}\text { RR } 0.80,95 \% \mathrm{Cl} 0.67 \text { to } 0.97 \text {, fewer assisted vaginal births } \\
\text { in the CSE group }\end{array}$ \\
\hline Caesarean section & $6(1015)$ & $\begin{array}{l}\text { RR } 1.04,95 \% \mathrm{Cl} 0.84 \text { to } 1.30 \text {, no evidence of a significant } \\
\text { difference between groups }\end{array}$ \\
\hline $\begin{array}{l}\text { Adverse effects for women (post dual } \\
\text { puncture) }\end{array}$ & $3(188)$ & $\begin{array}{l}\text { RR } 3.78,95 \% \mathrm{Cl} 0.16 \text { to } 89.09 \text {, no evidence of a significant } \\
\text { difference between groups }\end{array}$ \\
\hline $\begin{array}{l}\text { Adverse effects for women (known } \\
\text { dural tap) }\end{array}$ & $3(842)$ & $\begin{array}{l}\text { RR } 2.77,95 \% \mathrm{Cl} 0.66 \text { to } 11.65 \text {, no evidence of a significant } \\
\text { difference between groups }\end{array}$ \\
\hline Adverse effects for women (pruritus) & $6(370)$ & $\begin{array}{l}\text { Average RR } 7.34,95 \% \mathrm{Cl} 0.14 \text { to } 375.82 \text {, (random effects; } \\
\text { heterogeneity: } \mathrm{I}^{2}=97 \%, \mathrm{Tau}^{2}=15.29, \mathrm{Chi}^{2} \text { test for hetero- } \\
\text { geneity P <0.00001), there was no significant difference } \\
\text { between groups }\end{array}$ \\
\hline $\begin{array}{l}\text { Adverse effects for women (urinary re- } \\
\text { tention) }\end{array}$ & $1(704)$ & $\begin{array}{l}\mathrm{RR} 0.86,95 \% \mathrm{Cl} 0.79 \text { to } 0.95 \text {, fewer women in the CSE } \\
\text { group experienced urinary retention }\end{array}$ \\
\hline $\begin{array}{l}\text { Adverse effects for women (nau- } \\
\text { sea/vomiting) }\end{array}$ & $6(370)$ & $\begin{array}{l}\text { Average RR } 1.48,95 \% \mathrm{Cl} 0.55 \text { to } 3.95 \text {, (random effects; het- } \\
\text { erogeneity: }\left.\right|^{2}=59 \% \text {, } \mathrm{Tau}^{2}=0.51 \text {, Chi }{ }^{2} \text { test for heterogene- } \\
\text { ity } \mathrm{P}=0.06 \text { ), no significant difference between groups }\end{array}$ \\
\hline $\begin{array}{l}\text { Adverse effects for women (hypoten- } \\
\text { sion) }\end{array}$ & $6(1002)$ & $\begin{array}{l}\text { Average RR } 0.81,95 \% \mathrm{Cl} 0.65 \text { to } 1.02 \text {, no significant differ- } \\
\text { ence between groups }\end{array}$ \\
\hline
\end{tabular}


Table 28. (6.) Results by individual review - combined spinal epidural (Continued)

\begin{tabular}{|c|c|c|c|}
\hline & Adverse effects for women (headache) & $1(79)$ & $\begin{array}{l}\text { RR } 1.03,0.07 \text { to } 15.83 \text {, no significant difference between } \\
\text { groups }\end{array}$ \\
\hline & Adverse effects for women (sedation) & $1(79)$ & $\begin{array}{l}\text { RR } 1.03,0.46 \text { to } 2.31 \text {, no significant difference between } \\
\text { groups }\end{array}$ \\
\hline & Adverse effects for infants & & Outcome not reported in suitable format \\
\hline & $\begin{array}{l}\text { Admission to special care baby unit/ } \\
\text { neonatal intensive care }\end{array}$ & $1(704)$ & $\begin{array}{l}\text { RR } 0.63,95 \% \mathrm{Cl} 0.29 \text { to } 1.37 \text {, no significant difference be- } \\
\text { tween groups }\end{array}$ \\
\hline & $\begin{array}{l}\text { Apgar score less than seven at five min- } \\
\text { utes }\end{array}$ & $3(842)$ & $\begin{array}{l}\text { RR } 2.10,95 \% \mathrm{Cl} 0.63 \text { to } 6.97 \text {, no evidence of a significant } \\
\text { difference between groups }\end{array}$ \\
\hline & $\begin{array}{l}\text { Poor infant outcomes at long-term fol- } \\
\text { low-up }\end{array}$ & & Outcome not reported \\
\hline & Cost & & Outcome not reported \\
\hline \multirow{11}{*}{$\begin{array}{l}\text { Combined } \\
\text { spinal- } \\
\text { epidural } \\
\text { versus low- } \\
\text { dose epidur- } \\
\text { al }\end{array}$} & Outcome & $\begin{array}{l}\text { No. of stud- } \\
\text { ies (no. } \\
\text { women) }\end{array}$ & Results \\
\hline & $\begin{array}{l}\text { Pain intensity - time from first injec- } \\
\text { tion to effective analgesia in minutes }\end{array}$ & $5(461)$ & $\begin{array}{l}\text { Average Mean difference MD }-5.42,95 \% \mathrm{Cl}-7.26 \text { to }-3.59 \text {, } \\
\text { (random effects; heterogeneity: } 1^{2}=77 \%, \mathrm{Tau}^{2}=3.27, \mathrm{Chi}^{2} \\
\text { test for heterogeneity } \mathrm{P}=0.002 \text { ), CSE had a faster onset } \\
\text { time of effective pain relief from time of injection (approx- } \\
\text { imately } 6 \text { minutes) }\end{array}$ \\
\hline & $\begin{array}{l}\text { Pain intensity - number of women with } \\
\text { effective analgesia } 10 \text { minutes after } \\
\text { first injection }\end{array}$ & $1(101)$ & $\begin{array}{l}\mathrm{RR} 1.94,95 \% \mathrm{Cl} 1.49 \text { to } 2.54 \text {, more women in the CSE } \\
\text { group had effective analgesia }\end{array}$ \\
\hline & Satisfaction with pain relief & $7(520)$ & $\begin{array}{l}\text { RR } 1.01,95 \% \mathrm{Cl} 0.98 \text { to } 1.05 \text {, no significant difference be- } \\
\text { tween groups }\end{array}$ \\
\hline & Sense of control in labour & & Outcome not reported \\
\hline & Satisfaction with childbirth experience & & Outcome not reported \\
\hline & Effect on mother/baby interaction & & Outcome not reported \\
\hline & Breastfeeding & & Outcome not reported \\
\hline & Assisted vaginal birth & $11(1612)$ & $\begin{array}{l}\text { RR } 1.07,95 \% \mathrm{Cl} 0.88 \text { to } 1.30 \text {, no evidence of a significant } \\
\text { difference between groups }\end{array}$ \\
\hline & Caesarean section & $15(1960)$ & $\begin{array}{l}\text { RR } 0.97,95 \% \mathrm{Cl} 0.82 \text { to } 1.16 \text {, no evidence of a significant } \\
\text { difference between groups }\end{array}$ \\
\hline & $\begin{array}{l}\text { Adverse effects for women (post dual } \\
\text { puncture) }\end{array}$ & $9(701)$ & $\begin{array}{l}\text { RR } 1.68,95 \% \mathrm{Cl} 0.42 \text { to } 6.81 \text {, no evidence of a significant } \\
\text { difference between groups }\end{array}$ \\
\hline
\end{tabular}


Table 28. (6.) Results by individual review - combined spinal epidural (Continued)

\begin{tabular}{|c|c|c|}
\hline $\begin{array}{l}\text { Adverse effects for women (known } \\
\text { dural tap) }\end{array}$ & $6(1326)$ & $\begin{array}{l}\text { RR } 0.81,95 \% \mathrm{Cl} 0.22 \text { to } 2.98 \text {, no evidence of a significant } \\
\text { difference between groups }\end{array}$ \\
\hline $\begin{array}{l}\text { Adverse effects for women (number of } \\
\text { women requiring blood patch for post } \\
\text { dural puncture headache) }\end{array}$ & $7(531)$ & $\begin{array}{l}\text { RR } 2.22,95 \% \mathrm{Cl} 0.51 \text { to } 9.64 \text {, no evidence of a significant } \\
\text { difference between groups }\end{array}$ \\
\hline Adverse effects for women (pruritus) & $11(959)$ & $\begin{array}{l}\text { Average RR } 1.80,95 \% \mathrm{Cl} 1.22 \text { to } 2.65 \text {, (random effects; } \\
\text { heterogeneity: } \mathrm{I}^{2}=84 \%, \mathrm{Tau}^{2}=0.26 \text {, Chi }{ }^{2} \text { test for hetero- } \\
\text { geneity } \mathrm{P}<0.00001 \text { ), more women in the CSE group had } \\
\text { pruritus }\end{array}$ \\
\hline $\begin{array}{l}\text { Adverse effects for women (urinary re- } \\
\text { tention) }\end{array}$ & $4(964)$ & $\begin{array}{l}\text { RR } 1.05,95 \% \mathrm{Cl} 0.94 \text { to } 1.16 \text {, no evidence of a significant } \\
\text { difference between groups }\end{array}$ \\
\hline $\begin{array}{l}\text { Adverse effects for women (nau- } \\
\text { sea/vomiting) }\end{array}$ & $7(539)$ & $\begin{array}{l}\text { Average RR } 0.97,95 \% \mathrm{Cl} 0.65 \text { to } 1.45 \text {, no evidence of a sig- } \\
\text { nificant difference between groups }\end{array}$ \\
\hline $\begin{array}{l}\text { Adverse effects for women (hypoten- } \\
\text { sion) }\end{array}$ & $14(2040)$ & $\begin{array}{l}\text { Average RR } 1.35,95 \% \mathrm{Cl} 0.89 \text { to } 2.04 \text {, (random effects; het- } \\
\text { erogeneity: } \mathrm{I}^{2}=50 \%, \mathrm{Tau}^{2}=0.13, \mathrm{Chi}^{2} \text { test for heterogene- } \\
\text { ity } \mathrm{P}=0.04 \text { ), no evidence of a significant difference be- } \\
\text { tween groups }\end{array}$ \\
\hline $\begin{array}{l}\text { Adverse effects for women (headache } \\
\text { any) }\end{array}$ & $1(110)$ & $\begin{array}{l}\text { RR } 0.14,0.01 \text { to } 2.70 \text {, no evidence of a significant differ- } \\
\text { ence between groups }\end{array}$ \\
\hline Adverse effects for infants & & Outcome not reported in suitable format \\
\hline $\begin{array}{l}\text { Admission to special care baby unit/ } \\
\text { neonatal intensive care }\end{array}$ & $3(852)$ & $\begin{array}{l}\text { RR } 0.77,95 \% \mathrm{Cl} 0.34 \text { to } 1.73 \text {, no evidence of significant dif- } \\
\text { ference between groups }\end{array}$ \\
\hline $\begin{array}{l}\text { Apgar score less than seven at five min- } \\
\text { utes }\end{array}$ & $6(1092)$ & $\begin{array}{l}\text { RR } 0.70,95 \% \mathrm{Cl} 0.31 \text { to } 1.59 \text {, no evidence of a significant } \\
\text { difference between groups }\end{array}$ \\
\hline $\begin{array}{l}\text { Poor infant outcomes at long-term fol- } \\
\text { low-up }\end{array}$ & & Outcome not reported \\
\hline Cost & & Outcome not reported \\
\hline
\end{tabular}

$\mathrm{Cl}$ : confidence interval; MD: mean difference; RR: risk ratio

Table 29. Results by individual review - sterile water injection, Hutton 2009

Sterile water injection for labour pain: a systematic review and meta-analysis of randomised controlled trials 8 studies, 783 women (Hutton 2009)

\begin{tabular}{|c|c|c|c|}
\hline $\begin{array}{l}\text { Sterile wa- } \\
\text { ter injec- } \\
\text { tions versus } \\
\text { placebo or } \\
\text { other treat- } \\
\text { ment }\end{array}$ & Outcome & $\begin{array}{l}\text { No. of stud- } \\
\text { ies (no. } \\
\text { women) }\end{array}$ & Results \\
\hline
\end{tabular}

Pain intensity - VAS pain score at 10-30 4 (289) Average Mean difference MD -26.04, 95\% Cl -34.14.0 to minutes following intervention -17.94, (random effects: heterogeneity: $\left.\right|^{2}=65 \%$, Tau $^{2}=$ 41.69, Chi ${ }^{2}$ test for heterogeneity $\left.P=0.04\right)$, , a significant 
Table 29. Results by individual review - sterile water injection, Hutton 2009 (Continued)

reduction in pain score of the sterile water group compared with the placebo or other intervention group

\begin{tabular}{|c|c|c|}
\hline $\begin{array}{l}\text { Pain intensity - Pain intensity - VAS pain } \\
\text { score at } 45-60 \text { minutes following inter- } \\
\text { vention }\end{array}$ & $5(542)$ & $\begin{array}{l}\text { Average Mean difference MD }-36.27,95 \% \mathrm{Cl}-50.80 \text { to } \\
-21.74, \text { (random effects: heterogeneity: }\left.\right|^{2}=94 \% \text {, Tau }{ }^{2}= \\
255.58, \text { Chi' test for heterogeneity } \mathrm{P}<=0.00001 \text { ), a signif- } \\
\text { icant reduction in pain score of the sterile water group } \\
\text { compared with the placebo or other intervention group }\end{array}$ \\
\hline $\begin{array}{l}\text { Pain intensity - Pain intensity - VAS pain } \\
\text { score at 90-120 minutes following inter- } \\
\text { vention }\end{array}$ & $5(488)$ & $\begin{array}{l}\text { Average Mean difference MD }-27.74,95 \% \mathrm{Cl}-39.03 \text { to } \\
-16.45 \text {, (random effects: heterogeneity: } \mathrm{I}^{2}=86 \% \text {, Tau }{ }^{2}= \\
135.71 \text {, Chi2 test for heterogeneity } \mathrm{P}<=0.00001 \text { ), a signif- } \\
\text { icant reduction in pain score of the sterile water group } \\
\text { compared with the placebo or other intervention group }\end{array}$ \\
\hline Satisfaction with pain relief & & Outcome not reported \\
\hline Sense of control in labour & & Outcome not reported \\
\hline Satisfaction with childbirth experience & & Outcome not reported \\
\hline Effect on mother/baby interaction & & Outcome not reported \\
\hline Breastfeeding & & Outcome not reported \\
\hline Assisted vaginal birth & & Outcome not reported \\
\hline Caesarean section & $8(828)$ & $\begin{array}{l}\mathrm{RR} 0.51,95 \% \mathrm{Cl} 0.30 \text { to } 0.87 \text {, significantly fewer caesare- } \\
\text { an sections in the sterile water group compared with the } \\
\text { placebo or other intervention group }\end{array}$ \\
\hline Side effects for mother & & Outcome not reported \\
\hline Side effects for baby & & Outcome not reported \\
\hline $\begin{array}{l}\text { Admission to special care baby unit/ } \\
\text { neonatal intensive care }\end{array}$ & & Outcome not reported \\
\hline $\begin{array}{l}\text { Apgar score less than seven at five min- } \\
\text { utes }\end{array}$ & & Outcome not reported \\
\hline $\begin{array}{l}\text { Poor infant outcomes at long-term fol- } \\
\text { low-up }\end{array}$ & & Outcome not reported \\
\hline Cost & & Outcome not reported \\
\hline
\end{tabular}

$\mathrm{Cl}$ : confidence interval; MD: mean difference; RR: risk ratio; VAS: visual analogue scale

Table 30. Results by individual review - epidural ropivacaine vs bupivacaine, Halpern 2003

Epidural ropivacaine versus bupivacaine for labor: a meta-analysis, 23 studies, 2074 women (Halpern 2003a)

\begin{tabular}{lll}
\hline $\begin{array}{l}\text { Ropivacaine } \\
\text { versus bupi- }\end{array}$ & Outcome & No. of stud- \\
vacaine & ies (no. & women)
\end{tabular}


Table 30. Results by individual review - epidural ropivacaine vs bupivacaine, Halpern 2003 (Continued)
Pain intensity - time from first injection to ef- $\quad 9$ (755)
MD $0.66,95 \% \mathrm{Cl}-1.0$ to 2.31 , no evidence of a sig-
fective analgesia in minutes
nificant difference between groups

\begin{tabular}{|c|c|c|}
\hline Satisfaction with pain relief & $11(672)$ & $\begin{array}{l}\text { OR } 1.07,95 \% \mathrm{Cl} 0.73 \text { to } 1.59 \text {, no evidence of a sig- } \\
\text { nificant difference between groups }\end{array}$ \\
\hline Sense of control in labour & & Outcome not reported \\
\hline Satisfaction with childbirth experience & & Outcome not reported \\
\hline Effect on mother/baby interaction & & Outcome not reported \\
\hline Breastfeeding & & Outcome not reported \\
\hline Assisted vaginal birth & $18(1787)$ & $\begin{array}{l}\text { OR } 0.89,95 \% \mathrm{Cl} 0.67 \text { to } 1.18 \text {, no evidence of a sig- } \\
\text { nificant difference between groups }\end{array}$ \\
\hline Caesarean section & $19(1831)$ & $\begin{array}{l}\text { OR } 0.88,95 \% \mathrm{Cl} 0.67 \text { to } 1.14 \text {, no evidence of a sig- } \\
\text { nificant difference between groups }\end{array}$ \\
\hline Side effects for mother (hypotension) & $9(615)$ & $\begin{array}{l}\text { OR } 1.04,95 \% \mathrm{Cl} 0.60 \text { to } 1.8 \text {, no evidence of a signifi- } \\
\text { cant difference between groups }\end{array}$ \\
\hline Side effects for mother (nausea or vomiting) & $7(888)$ & $\begin{array}{l}\text { OR } 0.88,95 \% \mathrm{Cl} 0.59 \text { to } 1.29 \text {, no evidence of a sig- } \\
\text { nificant difference between groups }\end{array}$ \\
\hline Side effects for baby & & Outcome not reported in suitable format \\
\hline $\begin{array}{l}\text { Admission to special care baby unit/neonatal } \\
\text { intensive care }\end{array}$ & & Outcome not reported \\
\hline Apgar score less than seven at five minutes & $15(1550)$ & $\begin{array}{l}\text { OR } 0.99,95 \% \mathrm{Cl} 0.49 \text { to } 2.0 \text {, no evidence of a signifi- } \\
\text { cant difference between groups }\end{array}$ \\
\hline Poor infant outcomes at long-term follow-up & & Outcome not reported \\
\hline Cost & & Outcome not reported \\
\hline
\end{tabular}

Cl: confidence interval; MD: mean difference; OR: odds ratio

Table 31. Results by individual review - intrathecal opioids - Mardirosoff 2002

Fetal bradycardia due to intrathecal opioids for labour analgesia: a systematic review, 24 studies, 3513 women (Mardirosoff 2002)

\begin{tabular}{llll}
\hline $\begin{array}{l}\text { Intrathecal } \\
\text { opioids ver- } \\
\text { sus non-in- } \\
\text { trathecal } \\
\text { opioids }\end{array}$ & Outcome & $\begin{array}{l}\text { No. of stud- } \\
\text { ies (no. } \\
\text { women) }\end{array}$ & Results \\
\hline & Pain intensity & Outcome not reported \\
\hline & Satisfaction with pain relief & Outcome not reported \\
\hline
\end{tabular}


Table 31. Results by individual review - intrathecal opioids - Mardirosoff 2002 (Continued)

\begin{tabular}{|c|c|c|c|}
\hline & \multicolumn{2}{|l|}{ Sense of control in labour } & Outcome not reported \\
\hline & \multicolumn{2}{|l|}{ Satisfaction with childbirth experience } & Outcome not reported \\
\hline & \multicolumn{2}{|l|}{ Effect on mother/baby interaction } & Outcome not reported \\
\hline & \multicolumn{2}{|l|}{ Breastfeeding } & Outcome not reported \\
\hline & Assisted vaginal birth & $15(2831)$ & $\begin{array}{l}\mathrm{RR} 0.94,95 \% \mathrm{Cl} 0.83 \text { to } 1.07 \text {, no evidence of a signifi- } \\
\text { cant difference between groups }\end{array}$ \\
\hline & Caesarean section & $17(2954)$ & $\begin{array}{l}\mathrm{RR} 1.03,95 \% \mathrm{Cl} 0.87 \text { to } 1.21 \text {, no evidence of a signifi- } \\
\text { cant difference between groups }\end{array}$ \\
\hline $\begin{array}{l}\text { Opioids in } \\
\text { controls }\end{array}$ & Adverse effects for women (pruritus) & $9(1308)$ & $\begin{array}{l}\text { Average RR } 1.71,95 \% \mathrm{Cl} 0.97 \text { to } 3.02 \text {, no evidence of } \\
\text { a significant difference between groups }\end{array}$ \\
\hline \multirow[t]{7}{*}{$\begin{array}{l}\text { No opioids in } \\
\text { controls }\end{array}$} & Adverse effects for women (pruritus) & $11(855)$ & $\begin{array}{l}\mathrm{RR} 29.6,95 \% \mathrm{Cl} 13.6 \text { to } 64.6 \text {, significantly more pruri- } \\
\text { tus in women in the intrathecal opioid group }\end{array}$ \\
\hline & $\begin{array}{l}\text { Adverse effects for infants (fetal heart rate } \\
\text { abnormalities) }\end{array}$ & $17(2081)$ & $\begin{array}{l}\mathrm{RR} 1.17,95 \% \mathrm{Cl} 0.87 \text { to } 1.57 \text {, no evidence of a signifi- } \\
\text { cant difference between groups }\end{array}$ \\
\hline & $\begin{array}{l}\text { Adverse effects for infants (fetal bradycardia } \\
\text { within } 1 \text { hour) }\end{array}$ & $9(927)$ & $\begin{array}{l}\text { OR } 1.81,95 \% \mathrm{Cl} 1.04 \text { to } 3.14 \text {, significant increase in } \\
\text { fetal bradycardia with intrathecal opioids }\end{array}$ \\
\hline & \multicolumn{2}{|l|}{$\begin{array}{l}\text { Admission to special care baby unit/neona- } \\
\text { tal intensive care }\end{array}$} & Outcome not reported \\
\hline & Apgar score less than seven at five minutes & $11(1623)$ & $\begin{array}{l}\text { OR } 1.17,95 \% \mathrm{Cl} 0.44 \text { to } 3.11 \text {, no evidence of a signifi- } \\
\text { cant difference between groups }\end{array}$ \\
\hline & \multicolumn{2}{|l|}{$\begin{array}{l}\text { Poor infant outcomes at long-term fol- } \\
\text { low-up }\end{array}$} & Outcome not reported \\
\hline & \multicolumn{2}{|l|}{ Cost } & Outcome not reported \\
\hline
\end{tabular}

$\mathrm{Cl}$ : confidence interval; OR: odds ratio; RR: risk ratio

Table 32. Summary of outcomes reported in Cochrane systematic reviews - pharmacological interventions

\begin{tabular}{|c|c|c|c|c|c|c|}
\hline & $\begin{array}{l}\text { Inhaled anal- } \\
\text { gesia (Klomp } \\
\text { 2012) }\end{array}$ & $\begin{array}{l}\text { Parenteral opi- } \\
\text { oids review (Ull- } \\
\text { man 2010) }\end{array}$ & $\begin{array}{l}\text { Non-opioid drugs } \\
\text { (Othman 2012) }\end{array}$ & $\begin{array}{l}\text { Local anaes- } \\
\text { thetic nerve } \\
\text { blocks } \\
\text { (Novikova } \\
\text { 2012) }\end{array}$ & $\begin{array}{l}\text { Epidural (Anim-So- } \\
\text { muah 2011) }\end{array}$ & $\begin{array}{l}\text { Combined } \\
\text { spinal epidur- } \\
\text { al (Simmons } \\
\text { 2012) }\end{array}$ \\
\hline \multirow[t]{2}{*}{$\begin{array}{l}\text { Pain in- } \\
\text { tensity }\end{array}$} & $\begin{array}{l}9 / 26 \text { trials } \\
(26 \%) \text { reported } \\
\text { this outcome }\end{array}$ & $\begin{array}{l}28 / 57 \text { trials } \\
(49 \%) \text { reported } \\
\text { this outcome }\end{array}$ & $\begin{array}{l}2 / 18 \text { trials }(11 \%) \\
\text { reported this out- } \\
\text { come }\end{array}$ & $\begin{array}{l}\text { NOT REPORT- } \\
\text { ED }\end{array}$ & \multirow{2}{*}{$\begin{array}{l}7 / 38 \text { trials ( } 18 \% \text { ) re- } \\
\text { ported this outcome } \\
\text { Significant findings } \\
\text { observed for } 3 \text { com- } \\
\text { parisons ( } 7 \text { studies) }\end{array}$} & $\begin{array}{l}8 / 27 \text { trials } \\
(30 \%) \text { reported } \\
\text { this outcome }\end{array}$ \\
\hline & $\begin{array}{l}\text { Significant find- } \\
\text { ings observed } \\
\text { for } 4 \text { compar- } \\
\text { isons ( } 7 \text { stud- } \\
\text { ies) }\end{array}$ & $\begin{array}{l}\text { Significant find- } \\
\text { ings observed for }\end{array}$ & $\begin{array}{l}\text { Significant find- } \\
\text { ings observed for } \\
1 \text { comparison ( } 1 \\
\text { study) }\end{array}$ & & & $\begin{array}{l}\text { Significant find- } \\
\text { ings observed } \\
\text { for } 3 \text { compar- } \\
\text { isons ( } 8 \text { stud- } \\
\text { ies) }\end{array}$ \\
\hline
\end{tabular}


Table 32. Summary of outcomes reported in Cochrane systematic reviews - pharmacological interventions (Continued) 5 comparisons ( 8 studies)

\begin{tabular}{|c|c|c|c|c|c|c|}
\hline $\begin{array}{l}\text { Satisfac- } \\
\text { tion with } \\
\text { pain relief }\end{array}$ & $\begin{array}{l}8 / 26 \text { trials } \\
(31 \%) \text { reported } \\
\text { this outcome } \\
\text { No differences } \\
\text { observed be- } \\
\text { tween groups }\end{array}$ & $\begin{array}{l}12 / 57 \text { trials } \\
(21 \%) \\
\text { reported this } \\
\text { outcome } \\
\text { Significant find- } \\
\text { ings observed for } \\
2 \text { comparisons ( } 2 \\
\text { studies) }\end{array}$ & $\begin{array}{l}9 / 18 \text { trials }(50 \%) \\
\text { reported this out- } \\
\text { come } \\
\text { Significant find- } \\
\text { ings observed for } \\
5 \text { comparisons ( } 7 \\
\text { studies) }\end{array}$ & $\begin{array}{l}6 / 12 \text { trials } \\
(50 \%) \text { report- } \\
\text { ed this out- } \\
\text { come } \\
\text { Significant } \\
\text { findings ob- } \\
\text { served for } 2 \\
\text { comparisons } \\
\text { ( } 2 \text { studies) }\end{array}$ & $\begin{array}{l}9 / 38 \text { trials }(24 \%) \text { re- } \\
\text { ported this outcome } \\
\text { No differences ob- } \\
\text { served between } \\
\text { groups }\end{array}$ & $\begin{array}{l}7 / 27 \text { trials } \\
(26 \%) \text { reported } \\
\text { this outcome } \\
\text { No differences } \\
\text { observed be- } \\
\text { tween groups }\end{array}$ \\
\hline $\begin{array}{l}\text { Sense of } \\
\text { control in } \\
\text { labour }\end{array}$ & $\begin{array}{l}\text { NOT REPORT- } \\
\text { ED }\end{array}$ & NOT REPORTED & NOT REPORTED & $\begin{array}{l}\text { NOT REPORT- } \\
\text { ED }\end{array}$ & $\begin{array}{l}1 / 38 \text { trials }(3 \%) \text { re- } \\
\text { ported this outcome } \\
\text { No differences ob- } \\
\text { served between } \\
\text { groups }\end{array}$ & NOT REPORTED \\
\hline $\begin{array}{l}\text { Satisfac- } \\
\text { tion with } \\
\text { childbirth } \\
\text { experi- } \\
\text { ence }\end{array}$ & $\begin{array}{l}\text { NOT REPORT- } \\
\text { ED }\end{array}$ & $\begin{array}{l}1 / 57 \text { trial } \\
(1.75 \%) \text { reported } \\
\text { this outcome } \\
\text { No differences } \\
\text { observed be- } \\
\text { tween groups }\end{array}$ & $\begin{array}{l}1 / 18 \text { trials }(6 \%) \text { re- } \\
\text { ported this out- } \\
\text { come } \\
\text { Significant find- } \\
\text { ings observed for } \\
1 \text { comparison (1 } \\
\text { study) }\end{array}$ & $\begin{array}{l}\text { NOT REPORT- } \\
\text { ED }\end{array}$ & $\begin{array}{l}1 / 38 \text { trials }(3 \%) \text { re- } \\
\text { ported this outcome } \\
\text { No differences ob- } \\
\text { served between } \\
\text { groups }\end{array}$ & $\begin{array}{l}\text { NOT REPORT- } \\
\text { ED }\end{array}$ \\
\hline $\begin{array}{l}\text { Effect on } \\
\text { moth- } \\
\text { er/baby } \\
\text { interac- } \\
\text { tion }\end{array}$ & $\begin{array}{l}\text { NOT REPORT- } \\
\text { ED }\end{array}$ & NOT REPORTED & NOT REPORTED & $\begin{array}{l}\text { NOT REPORT- } \\
\text { ED }\end{array}$ & NOT REPORTED & NOT REPORTED \\
\hline $\begin{array}{l}\text { Breast- } \\
\text { feeding }\end{array}$ & $\begin{array}{l}\text { NOT REPORT- } \\
\text { ED }\end{array}$ & $\begin{array}{l}2 / 57 \text { trials }(3.5 \%) \\
\text { reported this } \\
\text { outcome }\end{array}$ & $\begin{array}{l}1 / 18 \text { trials }(6 \%) \text { re- } \\
\text { ported this out- } \\
\text { come } \\
\text { No differences ob- } \\
\text { served between } \\
\text { groups }\end{array}$ & $\begin{array}{l}\text { NOT REPORT- } \\
\text { ED }\end{array}$ & NOT REPORTED & NOT REPORTED \\
\hline $\begin{array}{l}\text { Assisted } \\
\text { vaginal } \\
\text { birth }\end{array}$ & $\begin{array}{l}7 / 26 \text { trials } \\
(27 \%) \text { reported } \\
\text { this outcome } \\
\text { No differences } \\
\text { observed be- } \\
\text { tween groups }\end{array}$ & $\begin{array}{l}17 / 57 \text { trials } \\
(30 \%) \\
\text { reported this } \\
\text { outcome }\end{array}$ & $\begin{array}{l}7 / 18 \text { trials (39\%) } \\
\text { reported this out- } \\
\text { come } \\
\text { No differences ob- } \\
\text { served between } \\
\text { groups }\end{array}$ & $\begin{array}{l}3 / 12 \text { trials } \\
(25 \%) \text { report- } \\
\text { ed this out- } \\
\text { come } \\
\text { No differences } \\
\text { observed be- } \\
\text { tween groups }\end{array}$ & $\begin{array}{l}23 / 38 \text { trials ( } 60 \%) \text { re- } \\
\text { ported this outcome } \\
\text { Significant findings } \\
\text { observed for } 1 \text { com- } \\
\text { parison ( } 23 \text { studies) }\end{array}$ & $\begin{array}{l}17 / 27 \text { trials } \\
(63 \%) \text { reported } \\
\text { this outcome } \\
\text { Significant find- } \\
\text { ings observed } \\
\text { for } 1 \text { compari- } \\
\text { son ( } 6 \text { studies) }\end{array}$ \\
\hline
\end{tabular}


Table 32. Summary of outcomes reported in Cochrane systematic reviews - pharmacological interventions (Continued)

\begin{tabular}{|c|c|c|c|c|c|c|}
\hline $\begin{array}{l}\text { Caesarean } \\
\text { section }\end{array}$ & $\begin{array}{l}6 / 26 \text { trials } \\
(23 \%) \text { reported } \\
\text { this outcome } \\
\text { No differences } \\
\text { observed be- } \\
\text { tween groups }\end{array}$ & $\begin{array}{l}20 / 57 \text { trials } \\
(35 \%) \\
\text { Reported this } \\
\text { outcome } \\
\text { No differences } \\
\text { observed be- } \\
\text { tween groups }\end{array}$ & $\begin{array}{l}3 / 18 \text { trials }(17 \%) \\
\text { reported this out- } \\
\text { come } \\
\text { No differences ob- } \\
\text { served between } \\
\text { groups }\end{array}$ & $\begin{array}{l}4 / 12 \text { trials } \\
(33 \%) \text { report- } \\
\text { ed this out- } \\
\text { come } \\
\text { No differences } \\
\text { observed be- } \\
\text { tween groups }\end{array}$ & $\begin{array}{l}27 / 38 \text { trials }(71 \%) \text { re- } \\
\text { ported this outcome } \\
\text { No differences ob- } \\
\text { served between } \\
\text { groups }\end{array}$ & $\begin{array}{l}21 / 27 \text { trials } \\
(78 \%) \text { reported } \\
\text { this outcome } \\
\text { No differences } \\
\text { observed be- } \\
\text { tween groups }\end{array}$ \\
\hline $\begin{array}{l}\text { Adverse } \\
\text { effects for } \\
\text { women }\end{array}$ & $\begin{array}{l}16 / 26 \text { trials } \\
(62 \%) \text { reported } \\
\text { this outcome } \\
\text { Significant find- } \\
\text { ings observed } \\
\text { for } 7 \text { compar- } \\
\text { isons ( } 9 \text { stud- } \\
\text { ies) }\end{array}$ & $\begin{array}{l}\text { Significant find- } \\
\text { ings observed for } \\
14 \text { comparisons } \\
\text { ( } 27 \text { studies) }\end{array}$ & $\begin{array}{l}6 / 18 \text { trials }(33 \%) \\
\text { reported this out- } \\
\text { come } \\
\text { No differences ob- } \\
\text { served between } \\
\text { groups }\end{array}$ & $\begin{array}{l}\text { 2/12 trials } \\
(17 \%) \text { report- } \\
\text { ed this out- } \\
\text { come } \\
\text { Significant } \\
\text { findings ob- } \\
\text { served for } 1 \\
\text { comparison (1 } \\
\text { study) }\end{array}$ & $\begin{array}{l}21 / 38 \text { trials }(55 \%) \text { re- } \\
\text { ported this outcome } \\
\text { Significant findings } \\
\text { observed for } 4 \text { com- } \\
\text { parisons ( } 12 \text { studies) }\end{array}$ & $\begin{array}{l}16 / 27 \text { trials } \\
(60 \%) \text { reported } \\
\text { this outcome } \\
\text { Significant find- } \\
\text { ings observed } \\
\text { for } 2 \text { compar- } \\
\text { isons ( } 12 \text { stud- } \\
\text { ies) }\end{array}$ \\
\hline $\begin{array}{l}\text { Adverse } \\
\text { effects for } \\
\text { infants }\end{array}$ & $\begin{array}{l}4 / 26 \text { trials } \\
(15 \%) \text { reported } \\
\text { this outcome } \\
\text { No differences } \\
\text { observed be- } \\
\text { tween groups }\end{array}$ & $\begin{array}{l}\text { Significant find- } \\
\text { ings observed for } \\
1 \text { comparison ( } 1 \\
\text { study) }\end{array}$ & $\begin{array}{l}5 / 18 \text { trials }(28 \%) \\
\text { reported this out- } \\
\text { come } \\
\text { No differences ob- } \\
\text { served between } \\
\text { groups }\end{array}$ & $\begin{array}{l}1 / 12 \text { trials } \\
(8 \%) \text { reported } \\
\text { this outcome } \\
\text { Significant } \\
\text { findings ob- } \\
\text { served for } 1 \\
\text { comparison ( } 1 \\
\text { study) }\end{array}$ & $\begin{array}{l}18 / 38 \text { trials ( } 47 \% \text { ) re- } \\
\text { ported this outcome } \\
\text { Significant findings } \\
\text { observed for } 2 \text { com- } \\
\text { parisons ( } 15 \text { studies) }\end{array}$ & NOT REPORTED \\
\hline $\begin{array}{l}\text { Admission } \\
\text { to special } \\
\text { care ba- } \\
\text { by unit/ } \\
\text { neonatal } \\
\text { intensive } \\
\text { care unit }\end{array}$ & $\begin{array}{l}\text { NOT REPORT- } \\
\text { ED }\end{array}$ & $\begin{array}{l}6 / 57 \text { trials }(10 \%) \\
\text { reported this } \\
\text { outcome } \\
\text { No differences } \\
\text { observed be- } \\
\text { tween groups }\end{array}$ & NOT REPORTED & $\begin{array}{l}\text { NOT REPORT- } \\
\text { ED }\end{array}$ & $\begin{array}{l}7 / 38 \text { trials }(18 \%) \text { re- } \\
\text { ported this outcome } \\
\text { No differences ob- } \\
\text { served between } \\
\text { groups }\end{array}$ & $\begin{array}{l}4 / 27 \text { trials } \\
(15 \%) \text { reported } \\
\text { this outcome } \\
\text { No differences } \\
\text { observed be- } \\
\text { tween groups }\end{array}$ \\
\hline $\begin{array}{l}\text { Apgar } \\
\text { score less } \\
\text { than sev- } \\
\text { en at five } \\
\text { minutes }\end{array}$ & $\begin{array}{l}7 / 26 \text { trials } \\
(27 \%) \text { reported } \\
\text { this outcome } \\
\text { No differences } \\
\text { observed be- } \\
\text { tween groups }\end{array}$ & $\begin{array}{l}12 / 57 \text { trials } \\
(21 \%) \text { reported } \\
\text { this outcome } \\
\text { No differences } \\
\text { observed be- } \\
\text { tween groups }\end{array}$ & $\begin{array}{l}4 / 18 \text { trials }(22 \%) \\
\text { reported this out- } \\
\text { come } \\
\text { No differences ob- } \\
\text { served between } \\
\text { groups }\end{array}$ & $\begin{array}{l}4 / 12 \text { trials } \\
(33 \%) \text { report- } \\
\text { ed this out- } \\
\text { come } \\
\text { No differences } \\
\text { observed be- } \\
\text { tween groups }\end{array}$ & $\begin{array}{l}18 / 38 \text { trials }(47 \%) \text { re- } \\
\text { ported this outcome } \\
\text { No differences ob- } \\
\text { served between } \\
\text { groups }\end{array}$ & $\begin{array}{l}9 / 27 \text { trials } \\
(33 \%) \text { reported } \\
\text { this outcome } \\
\text { No differences } \\
\text { observed be- } \\
\text { tween groups }\end{array}$ \\
\hline $\begin{array}{l}\text { Poor in- } \\
\text { fant out- } \\
\text { comes at } \\
\text { long-term } \\
\text { follow-up }\end{array}$ & $\begin{array}{l}\text { NOT REPORT- } \\
\text { ED }\end{array}$ & NOT REPORTED & NOT REPORTED & $\begin{array}{l}\text { NOT REPORT- } \\
\text { ED }\end{array}$ & NOT REPORTED & NOT REPORTED \\
\hline
\end{tabular}


Table 32. Summary of outcomes reported in Cochrane systematic reviews - pharmacological interventions (Continued)

Cost NOT REPORT- $\quad$ NOT REPORTED NOT REPORTED ED

NOT REPORT- $\quad$ NOT REPORTED

NOT REPORTED ED 
Table 33. Summary of outcomes reported in Cochrane systematic reviews - non-pharmacological interventions

\begin{tabular}{|c|c|c|c|c|c|c|c|c|c|}
\hline & $\begin{array}{l}\text { Hypnosis (Mad- } \\
\text { den } 2012\end{array}$ & $\begin{array}{l}\text { Biofeed- } \\
\text { back } \\
\text { (Bar- } \\
\text { ragán } \\
2011 \text { ) }\end{array}$ & $\begin{array}{l}\text { Sterile wa- } \\
\text { ter injection } \\
\text { (Derry 2012) }\end{array}$ & $\begin{array}{l}\text { Immersion in water } \\
\text { (Cluett 2009) }\end{array}$ & $\begin{array}{l}\text { Aro- } \\
\text { mathera- } \\
\text { py (Smith } \\
2011 c)\end{array}$ & $\begin{array}{l}\text { Relaxation } \\
\text { techniques } \\
\text { (Smith 2011b) }\end{array}$ & $\begin{array}{l}\text { Acupuncture } \\
\text { or acupressure } \\
\text { (Smith 2011a) }\end{array}$ & $\begin{array}{l}\text { Massage, re- } \\
\text { flexology \& } \\
\text { other manual } \\
\text { methods (Smith } \\
\text { 2012) }\end{array}$ & $\begin{array}{l}\text { TENS } \\
\text { (Dowswell } \\
\text { 2009) }\end{array}$ \\
\hline $\begin{array}{l}\text { Pain in- } \\
\text { tensity }\end{array}$ & $\begin{array}{l}1 / 7 \text { trials (14\%) } \\
\text { reported this out- } \\
\text { come } \\
\text { Significant find- } \\
\text { ings observed for } \\
1 \text { comparison (1 } \\
\text { study) - but this } \\
\text { was a quasi-RCT }\end{array}$ & $\begin{array}{l}\text { NOT RE- } \\
\text { PORTED }\end{array}$ & $\begin{array}{l}3 / 7 \text { trials } \\
(43 \%) \text { re- } \\
\text { ported this } \\
\text { outcome } \\
\text { Significant } \\
\text { findings } \\
\text { observed } \\
\text { for } 10 \text { com- } \\
\text { parisons (3 } \\
\text { studies) }\end{array}$ & $\begin{array}{l}4 / 12 \text { trials ( } 33 \%) \text { re- } \\
\text { ported this outcome } \\
\text { Significant findings } \\
\text { observed for } 10 \text { com- } \\
\text { parisons (1 study) }\end{array}$ & $\begin{array}{l}\text { NOT RE- } \\
\text { PORTED }\end{array}$ & $\begin{array}{l}\text { 5/11 trials } \\
(45 \%) \text { report- } \\
\text { ed this out- } \\
\text { come } \\
\text { Significant } \\
\text { findings ob- } \\
\text { served for } 3 \\
\text { comparisons } \\
\text { ( } 3 \text { studies) }\end{array}$ & $\begin{array}{l}7 / 13 \text { trials (54\%) } \\
\text { reported this out- } \\
\text { come } \\
\text { Significant find- } \\
\text { ings observed for } \\
3 \text { comparison (4 } \\
\text { studies) }\end{array}$ & $\begin{array}{l}\text { Significant find- } \\
\text { ings observed } \\
\text { for } 2 \text { compar- } \\
\text { isons ( } 5 \text { studies) }\end{array}$ & $\begin{array}{l}10 / 17 \text { trials } \\
\text { (59\%) report- } \\
\text { ed this out- } \\
\text { come } \\
\text { Significant } \\
\text { findings ob- } \\
\text { served for } 2 \\
\text { comparisons } \\
\text { ( } 3 \text { studies) }\end{array}$ \\
\hline $\begin{array}{l}\text { Satisfac- } \\
\text { tion with } \\
\text { pain re- } \\
\text { lief }\end{array}$ & $\begin{array}{l}1 / 7 \text { trials }(14 \%) \\
\text { reported this out- } \\
\text { come } \\
\text { No differences } \\
\text { observed be- } \\
\text { tween groups }\end{array}$ & $\begin{array}{l}\text { NOT RE- } \\
\text { PORTED }\end{array}$ & $\begin{array}{l}\text { NOT RE- } \\
\text { PORTED }\end{array}$ & NOT ) & $\begin{array}{l}\text { NOT RE- } \\
\text { PORTED }\end{array}$ & $\begin{array}{l}3 / 11 \text { trials } \\
(27 \%) \text { report- } \\
\text { ed this out- } \\
\text { come } \\
\text { Significant } \\
\text { findings ob- } \\
\text { served for } 2 \\
\text { comparisons } \\
\text { ( } 2 \text { studies) }\end{array}$ & $\begin{array}{l}3 / 13 \text { trials }(23 \%) \\
\text { reported this out- } \\
\text { come } \\
\text { Significant find- } \\
\text { ings observed for } \\
2 \text { comparisons ( } 2 \\
\text { studies) }\end{array}$ & $\begin{array}{l}2 / 6 \text { trials }(33 \%) \\
\text { reported this } \\
\text { outcome }\end{array}$ & $\begin{array}{l}6 / 17 \text { trials } \\
(35 \%) \text { report- } \\
\text { ed this out- } \\
\text { come } \\
\text { Significant } \\
\text { findings ob- } \\
\text { served for } 1 \\
\text { comparison (1 } \\
\text { study) }\end{array}$ \\
\hline $\begin{array}{l}\text { Sense of } \\
\text { control } \\
\text { in labour }\end{array}$ & NOT REPORTED & $\begin{array}{l}\text { NOT RE- } \\
\text { PORTED }\end{array}$ & $\begin{array}{l}\text { NOT RE- } \\
\text { PORTED }\end{array}$ & NOT REPORTED & $\begin{array}{l}\text { NOT RE- } \\
\text { PORTED }\end{array}$ & $\begin{array}{l}\text { NOT REPORT- } \\
\text { ED }\end{array}$ & NOT REPORTED & $\begin{array}{l}1 / 6 \text { trials }(17 \%) \\
\text { reported this } \\
\text { outcome } \\
\text { No differences } \\
\text { observed be- } \\
\text { tween groups }\end{array}$ & $\begin{array}{l}\text { NOT REPORT- } \\
\text { ED }\end{array}$ \\
\hline $\begin{array}{l}\text { Satisfac- } \\
\text { tion with } \\
\text { child- } \\
\text { birth ex- } \\
\text { perience }\end{array}$ & $\begin{array}{l}2 / 7 \text { trials }(28 \%) \\
\text { reported this out- } \\
\text { come } \\
\text { No differences } \\
\text { observed be- } \\
\text { tween groups }\end{array}$ & $\begin{array}{l}\text { NOT RE- } \\
\text { PORTED }\end{array}$ & $\begin{array}{l}\text { NOT RE- } \\
\text { PORTED }\end{array}$ & $\begin{array}{l}1 / 12 \text { trial (8\%) re- } \\
\text { ported this outcome } \\
\text { No differences ob- } \\
\text { served between } \\
\text { groups }\end{array}$ & $\begin{array}{l}\text { NOT RE- } \\
\text { PORTED }\end{array}$ & $\begin{array}{l}2 / 11 \text { trials } \\
(18 \%) \text { report- } \\
\text { ed this out- } \\
\text { come } \\
\text { Significant } \\
\text { findings ob- }\end{array}$ & $\begin{array}{l}1 / 13 \text { trials }(7 \%) \\
\text { reported this out- } \\
\text { come } \\
\text { No differences } \\
\text { observed be- } \\
\text { tween groups }\end{array}$ & NOT REPORTED & $\begin{array}{l}\text { NOT REPORT- } \\
\text { ED }\end{array}$ \\
\hline
\end{tabular}




\begin{tabular}{|c|c|c|c|c|c|c|c|c|c|}
\hline & & & & & & $\begin{array}{l}\text { served for } 1 \\
\text { comparison (1 } \\
\text { study) }\end{array}$ & 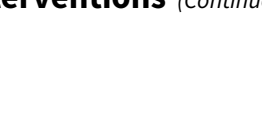 & & \\
\hline $\begin{array}{l}\text { Effect on } \\
\text { moth- } \\
\text { er/baby } \\
\text { interac- } \\
\text { tion }\end{array}$ & NOT REPORTED & $\begin{array}{l}\text { NOT RE- } \\
\text { PORTED }\end{array}$ & $\begin{array}{l}\text { NOT RE- } \\
\text { PORTED }\end{array}$ & NOT REPORTED & $\begin{array}{l}\text { NOT RE- } \\
\text { PORTED }\end{array}$ & $\begin{array}{l}\text { NOT REPORT- } \\
\text { ED }\end{array}$ & NOT REPORTED & NOT REPORTED & $\begin{array}{l}\text { NOT REPORT- } \\
\text { ED }\end{array}$ \\
\hline $\begin{array}{l}\text { Breast- } \\
\text { feeding }\end{array}$ & $\begin{array}{l}1 / 7 \text { trials (14\%) } \\
\text { reported this out- } \\
\text { come } \\
\text { No differences } \\
\text { observed be- } \\
\text { tween groups }\end{array}$ & $\begin{array}{l}\text { NOT RE- } \\
\text { PORTED }\end{array}$ & $\begin{array}{l}\text { NOT RE- } \\
\text { PORTED }\end{array}$ & $\begin{array}{l}3 / 12 \text { trials }(25 \%) \text { re- } \\
\text { ported this outcome } \\
\text { No differences ob- } \\
\text { served between } \\
\text { groups }\end{array}$ & $\begin{array}{l}\text { NOT RE- } \\
\text { PORTED }\end{array}$ & $\begin{array}{l}\text { NOT REPORT- } \\
\text { ED }\end{array}$ & NOT REPORTED & NOT REPORTED & $\begin{array}{l}\text { NOT REPORT- } \\
\text { ED }\end{array}$ \\
\hline $\begin{array}{l}\text { Assisted } \\
\text { vaginal } \\
\text { birth }\end{array}$ & $\begin{array}{l}3 / 7 \text { trials }(43 \%) \\
\text { reported this out- } \\
\text { come } \\
\text { No differences } \\
\text { observed be- } \\
\text { tween groups }\end{array}$ & $\begin{array}{l}2 / 4 \text { trials } \\
(50 \%) \text { re- } \\
\text { ported } \\
\text { this out- } \\
\text { come } \\
\text { No dif- } \\
\text { ferences } \\
\text { ob- } \\
\text { served } \\
\text { between } \\
\text { groups }\end{array}$ & $\begin{array}{l}6 / 7 \text { trials } \\
(86 \%) \text { re- } \\
\text { ported this } \\
\text { outcome } \\
\text { No differ- } \\
\text { ences ob- } \\
\text { served } \\
\text { between } \\
\text { groups }\end{array}$ & $\begin{array}{l}8 / 12 \text { trials }(67 \%) \text { re- } \\
\text { ported this outcome } \\
\text { No differences ob- } \\
\text { served between } \\
\text { groups }\end{array}$ & $\begin{array}{l}2 / 2 \text { trials } \\
(100 \%) \\
\text { reported } \\
\text { this out- } \\
\text { come } \\
\text { No differ- } \\
\text { ences ob- } \\
\text { served } \\
\text { between } \\
\text { groups }\end{array}$ & $\begin{array}{l}3 / 11 \text { trials } \\
(27 \%) \text { report- } \\
\text { ed this out- } \\
\text { come } \\
\text { Significant } \\
\text { findings ob- } \\
\text { served for } 1 \\
\text { comparison ( } 2 \\
\text { studies) }\end{array}$ & $\begin{array}{l}7 / 13 \text { trials }(54 \%) \\
\text { reported this out- } \\
\text { come } \\
\text { Significant find- } \\
\text { ings observed for } \\
\text { one comparison } \\
\text { (1 study) }\end{array}$ & $\begin{array}{l}2 / 6 \text { trials }(33 \%) \\
\text { reported this } \\
\text { outcome }\end{array}$ & $\begin{array}{l}11 / 17 \text { trials } \\
(65 \%) \text { report- } \\
\text { ed this out- } \\
\text { come } \\
\text { Signficant } \\
\text { findings ob- } \\
\text { served for } 1 \\
\text { comparison (1 } \\
\text { study) }\end{array}$ \\
\hline $\begin{array}{l}\text { Caesare- } \\
\text { an sec- } \\
\text { tion }\end{array}$ & $\begin{array}{l}3 / 7 \text { trials }(43 \%) \\
\text { reported this out- } \\
\text { come } \\
\text { No differences } \\
\text { observed be- } \\
\text { tween groups }\end{array}$ & $\begin{array}{l}2 / 4 \text { trials } \\
(50 \%) \text { re- } \\
\text { ported } \\
\text { this out- } \\
\text { come } \\
\text { No dif- } \\
\text { ferences } \\
\text { ob- } \\
\text { served } \\
\text { between } \\
\text { groups }\end{array}$ & $\begin{array}{l}7 / 7 \text { trials } \\
(100 \%) \text { re- } \\
\text { ported this } \\
\text { outcome } \\
\text { No differ- } \\
\text { ences ob- } \\
\text { served } \\
\text { between } \\
\text { groups }\end{array}$ & $\begin{array}{l}9 / 12 \text { trials }(75 \%) \text { re- } \\
\text { ported this outcome } \\
\text { No differences ob- } \\
\text { served between } \\
\text { groups }\end{array}$ & $\begin{array}{l}2 / 2 \text { trials } \\
(100 \%) \\
\text { reported } \\
\text { this out- } \\
\text { come } \\
\text { No differ- } \\
\text { ences ob- } \\
\text { served } \\
\text { between } \\
\text { groups }\end{array}$ & $\begin{array}{l}4 / 11 \text { trials } \\
(36 \%) \text { report- } \\
\text { ed this out- } \\
\text { come } \\
\text { No differ- } \\
\text { ences ob- } \\
\text { served be- } \\
\text { tween groups }\end{array}$ & $\begin{array}{l}\text { 9/13 trials (69\%) } \\
\text { reported this out- } \\
\text { come } \\
\text { Significant find- } \\
\text { ings observed for } \\
\text { one comparison } \\
\text { (1 study) }\end{array}$ & $\begin{array}{l}2 / 6 \text { trials }(33 \%) \\
\text { reported this } \\
\text { outcome }\end{array}$ & $\begin{array}{l}13 / 17 \text { trials } \\
(76 \%) \text { report- } \\
\text { ed this out- } \\
\text { come } \\
\text { No differ- } \\
\text { ences ob- } \\
\text { served be- } \\
\text { tween groups }\end{array}$ \\
\hline
\end{tabular}




\begin{tabular}{|c|c|c|c|c|c|c|c|c|c|c|c|}
\hline 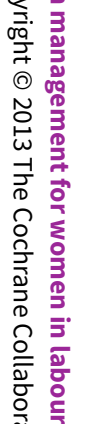 & $\begin{array}{l}\text { Ad- } \\
\text { verse ef- } \\
\text { fects for } \\
\text { women }\end{array}$ & $\begin{array}{l}1 / 7 \text { trials }(14 \%) \\
\text { reported this out- } \\
\text { come } \\
\text { No differences } \\
\text { observed be- } \\
\text { tween groups }\end{array}$ & $\begin{array}{l}\text { NOT RE- } \\
\text { PORTED }\end{array}$ & $\begin{array}{l}1 / 7 \text { trials } \\
(14 \%) \text { re- } \\
\text { ported this } \\
\text { outcome } \\
\text { Significant } \\
\text { findings } \\
\text { observed } \\
\text { for } 2 \text { com- } \\
\text { parisons (1 } \\
\text { study) }\end{array}$ & $\begin{array}{l}10 / 12 \text { trials ( } 83 \% \text { ) re- } \\
\text { ported this outcome } \\
\text { Significant findings } \\
\text { observed for } 3 \text { com- } \\
\text { parisons ( } 1 \text { study) }\end{array}$ & $\begin{array}{l}1 / 2 \text { trials } \\
(50 \%) \text { re- } \\
\text { ported } \\
\text { this out- } \\
\text { come } \\
\text { No differ- } \\
\text { ences ob- } \\
\text { served } \\
\text { between } \\
\text { groups }\end{array}$ & $\begin{array}{l}\text { NOT REPORT- } \\
\text { ED }\end{array}$ & NOT REPORTED & NOT REPORTED & $\begin{array}{l}\text { NOT REPORT- } \\
\text { ED }\end{array}$ & 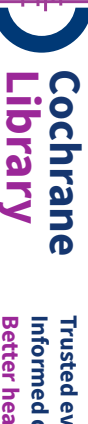 \\
\hline 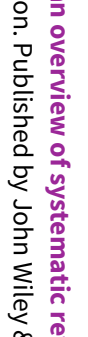 & $\begin{array}{l}\text { Adverse } \\
\text { effects } \\
\text { for in- } \\
\text { fants }\end{array}$ & $\begin{array}{l}1 / 7 \text { trials ( } 14 \%) \\
\text { reported this out- } \\
\text { come } \\
\text { No differences } \\
\text { observed be- } \\
\text { tween groups }\end{array}$ & $\begin{array}{l}\text { NOT RE- } \\
\text { PORTED }\end{array}$ & $\begin{array}{l}\text { NOT RE- } \\
\text { PORTED }\end{array}$ & $\begin{array}{l}10 / 12 \text { trials ( } 83 \%) \text { re- } \\
\text { ported this outcome } \\
\text { No differences ob- } \\
\text { served between } \\
\text { groups }\end{array}$ & $\begin{array}{l}\text { NOT RE- } \\
\text { PORTED }\end{array}$ & $\begin{array}{l}\text { NOT REPORT- } \\
\text { ED }\end{array}$ & NOT REPORTED & NOT REPORTED & $\begin{array}{l}2 / 17 \text { trials } \\
(12 \%) \text { report- } \\
\text { ed this out- } \\
\text { come } \\
\text { No differ- } \\
\text { ences ob- } \\
\text { served be- } \\
\text { tween groups }\end{array}$ & ?ำ \\
\hline 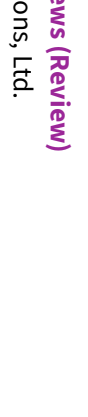 & $\begin{array}{l}\text { Admis- } \\
\text { sion to } \\
\text { special } \\
\text { care ba- } \\
\text { by unit/ } \\
\text { neonatal } \\
\text { intensive } \\
\text { care unit }\end{array}$ & $\begin{array}{l}2 / 7 \text { trials }(28 \%) \\
\text { reported this out- } \\
\text { come } \\
\text { No differences } \\
\text { observed be- } \\
\text { tween groups }\end{array}$ & $\begin{array}{l}\text { NOT RE- } \\
\text { PORTED }\end{array}$ & $\begin{array}{l}\text { NOT RE- } \\
\text { PORTED }\end{array}$ & $\begin{array}{l}4 / 12 \text { trials (33\%) re- } \\
\text { ported this outcome } \\
\text { No differences ob- } \\
\text { served between } \\
\text { groups }\end{array}$ & $\begin{array}{l}2 / 2 \text { trials } \\
(100 \%) \\
\text { reported } \\
\text { this out- } \\
\text { come } \\
\text { No differ- } \\
\text { ences ob- } \\
\text { served } \\
\text { between } \\
\text { groups }\end{array}$ & $\begin{array}{l}\text { NOT REPORT- } \\
\text { ED }\end{array}$ & NOT REPORTED & $\begin{array}{l}1 / 6 \text { trials (17\%) } \\
\text { reported this } \\
\text { outcome } \\
\text { No differences } \\
\text { observed be- } \\
\text { tween groups }\end{array}$ & $\begin{array}{l}\text { NOT REPORT- } \\
\text { ED }\end{array}$ & \\
\hline & $\begin{array}{l}\text { Apgar } \\
\text { score } \\
\text { less than } \\
\text { seven at } \\
\text { five min- } \\
\text { utes }\end{array}$ & $\begin{array}{l}1 / 7 \text { trials }(14 \%) \\
\text { reported this out- } \\
\text { come } \\
\text { No differences } \\
\text { observed be- } \\
\text { tween groups }\end{array}$ & $\begin{array}{l}\text { NOT RE- } \\
\text { PORTED }\end{array}$ & $\begin{array}{l}\text { NOT RE- } \\
\text { PORTED }\end{array}$ & $\begin{array}{l}7 / 12 \text { trials (58\%) re- } \\
\text { ported this outcome } \\
\text { No differences ob- } \\
\text { served between } \\
\text { groups }\end{array}$ & $\begin{array}{l}\text { NOT RE- } \\
\text { PORTED }\end{array}$ & $\begin{array}{l}\text { NOT REPORT- } \\
\text { ED }\end{array}$ & $\begin{array}{l}5 / 13 \text { trials ( } 38 \%) \\
\text { reported this out- } \\
\text { come } \\
\text { No differences } \\
\text { observed be- } \\
\text { tween groups }\end{array}$ & NOT REPORTED & $\begin{array}{l}\text { NOT REPORT- } \\
\text { ED }\end{array}$ & D \\
\hline E & $\begin{array}{l}\text { Poor in- } \\
\text { fant out- } \\
\text { comes }\end{array}$ & NOT REPORTED & $\begin{array}{l}\text { NOT RE- } \\
\text { PORTED }\end{array}$ & $\begin{array}{l}\text { NOT RE- } \\
\text { PORTED }\end{array}$ & NOT REPORTED & $\begin{array}{l}\text { NOT RE- } \\
\text { PORTED }\end{array}$ & $\begin{array}{l}\text { NOT REPORT- } \\
\text { ED }\end{array}$ & NOT REPORTED & NOT REPORTED & $\begin{array}{l}\text { NOT REPORT- } \\
\text { ED }\end{array}$ & . \\
\hline
\end{tabular}




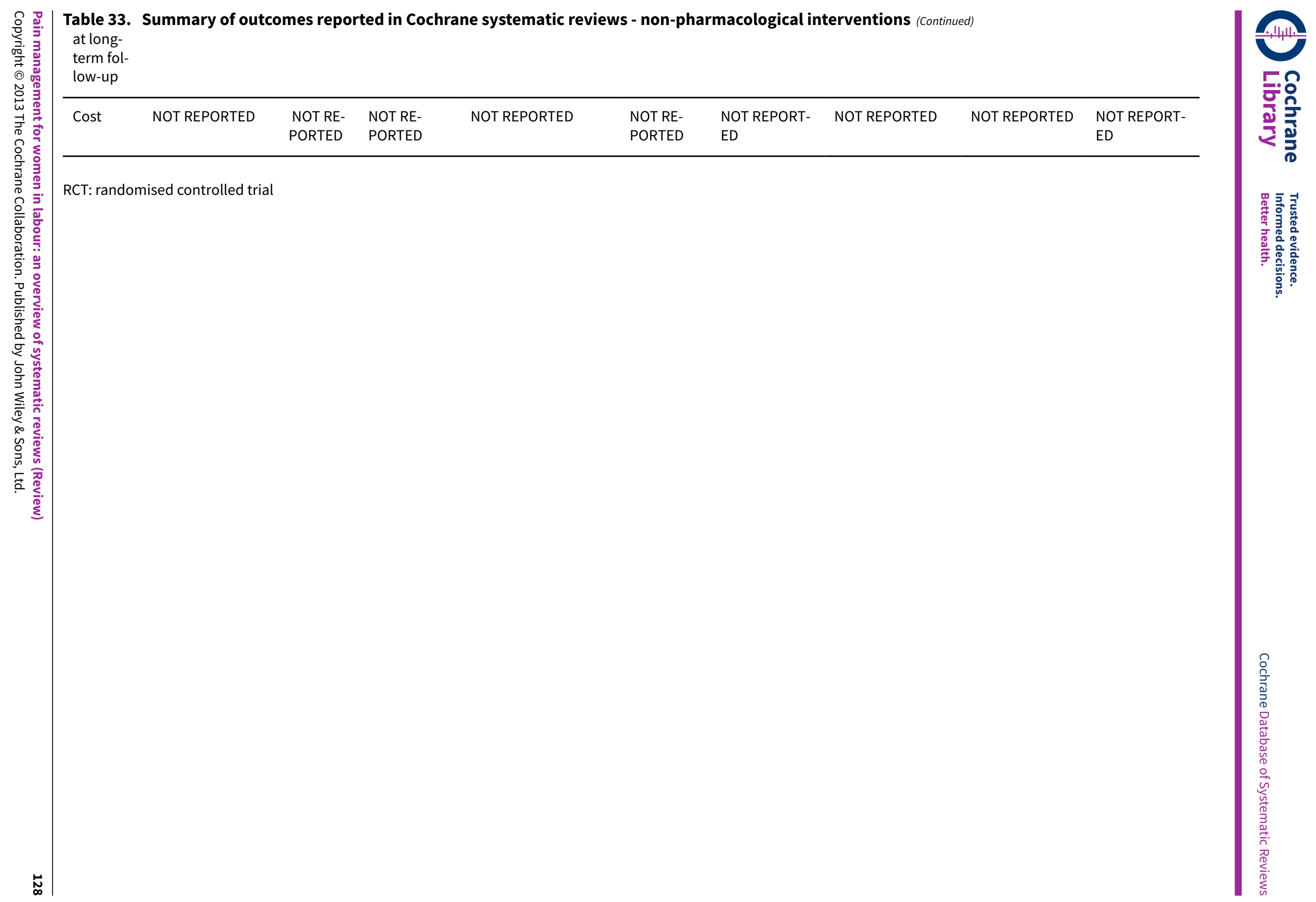




\section{APPENDICES}

\section{Appendix 1. Search strategies for non-Cochrane reviews}

Database of Abstracts of Reviews of Effects (The Cochrane Library 2011, Issue 2 of 4)

\#1 MeSH descriptor Labor Pain explode all trees

\#2 labor or labour or birth or childbirth

\#3 pain or analges*

\#4 MeSH descriptor Analgesia, Obstetrical explode all trees

\#5 (\#2 AND \#3)

\#6 (\#1 OR \#4 OR \#5)

MEDLINE (via OVID) (1966 to 31 May 2011)

1. ("review" or "review academic" or "review tutorial").pt.

2. (medline or medlars or embase or pubmed).tw,sh.

3. (scisearch or psychinfo or psycinfo).tw,sh.

4. (psychlit or psyclit).tw,sh.

5. cinahl.tw,sh.

6. ((hand adj2 search\$) or (manual\$ adj2 search\$)).tw,sh.

7. (electronic database\$ or bibliographic database\$ or computeri?ed database $\$$ or online database\$).tw,sh.

8. (pooling or pooled or mantel haenszel).tw,sh.

9. (retraction of publication or retracted publication).pt.

10.(peto or dersimonian or der simonian or fixed effect).tw,sh.

11.or/2-10

12.1 and 11

13.meta-analysis.pt.

14.meta-analysis.sh.

15. (meta-analys\$ or meta analys\$ or metaanalys\$).tw,sh.

16.(systematic\$ adj5 review\$).tw,sh.

17.(systematic $\$$ adj5 overview\$).tw,sh.

18. (quantitativ\$ adj5 review\$).tw,sh.

19. (quantitativ\$ adj5 overview\$).tw,sh.

20. (quantitativ\$ adj5 synthesis\$).tw,sh.

21. (methodologic\$ adj5 review\$).tw,sh.

22. (methodologic\$ adj5 overview\$).tw,sh.

23. (integrative research review\$ or research integration).tw.

24.or/13-23

25.12 or 24

26.exp Labor Pain/

27.exp Analgesia, Obstetrical/

28. (labor or labour or childbirth or birth).mp. and (pain* or analges*).tw.

29.exp Anesthesia, Obstetrical/

30.26 or 27 or 28 or 29

31.25 and 30

EMBASE (via NHS Evidence Health Information Resources) (1980 to 31 May 2011)

1. $\exp R E V I E W /$

2. (medline OR medlars OR embase OR pubmed).af

3. (psycinfo OR psychinfo).af

4. cinahl.af

5. (((hand adj2 search\$) OR (manual\$ ADJ search\$))).ti,ab

6. (((electronic ADJ database\$) OR (bibliographic ADJ database\$))).ti,ab

7. ((pooled ADJ analys\$) OR (pooling)).ti,ab

Pain management for women in labour: an overview of systematic reviews (Review) 
8. (peto OR dersimonian OR (fixed ADJ effect) OR mantel AND haenszel).ti,ab

9. RETRACTED ARTICLE/

10.(scisearch OR psychlit OR psyclit).af

11.2 OR 3 OR 4 OR 5 OR 6 OR 7 OR 8 OR 9 OR 10

12.1 AND 11

13.exp META ANALYSIS/

14.meta?analys $\$$.af

15. (systematic\$ adj5 review\$).af

16.(systematic\$ adj5 overview\$).af

17.(quantitativ\$ adj5 review\$).af

18. (quantitativ\$ adj5 overview\$).af

19. (methodologic\$ adj5 review\$).af

20.(methodologic\$ adj5 overview\$).af

21.((integrative adj5 research adj5 review\$) OR (research adj5 integration)).ti,ab

22. (quantitativ\$ adj5 synthesi\$).af

23.13 OR 14 OR 15 OR 16 OR 17 OR 18 OR 19 OR 20 OR 21 OR 22

24.12 OR 23

25. $\exp$ LABOR PAIN/

26. $\exp$ OBSTETRIC ANALGESIA/

27.exp OBSTETRIC ANESTHESIA/

28.((labor OR labour OR birth OR childbirth) AND (pain* OR analges $\left.\left.{ }^{\star}\right)\right) . t i, a b$

29.25 OR 26 OR 27 OR 28

30.24 AND 29

Systematic review search filters (lines 1-25 in the MEDLINE search and 1-24 in the EMBASE search) taken from the Clinical Evidence website. The EMBASE filter was adapted for the NHS Evidence Health Information Resources version of EMBASE.

WHAT'S NEW

\begin{tabular}{lll}
\hline Date & Event & Description \\
\hline 20 May 2013 & Amended & $\begin{array}{l}\text { Number of new (6) and updated (9) Cochrane reviews corrected } \\
\text { in the Abstract. NIHR acknowledgement added. }\end{array}$ \\
\hline
\end{tabular}

\section{CONTRIBUTIONS OFAUTHORS}

James Neilson and Zarko Alfirevic conceived the idea of the overview. Leanne Jones, Mohammad Othman and Therese Dowswell assessed review eligibility and carried out all data extraction and data entry. Therese Dowswell and Leanne Jones co-authored initial drafts of the overview. James Neilson, Zarko Alfirevic, Simon Gates, Mary Newburn, Sue Jordan and Tina Lavender commented on draft versions of the protocol and the final overview.

\section{DECLARATIONSOF INTEREST}

None known.

\section{SOURCES OF SUPPORT}

\section{Internal sources}

- The University of Liverpool, UK.

\section{External sources}

- National Institute for Health Research, UK.

Cochrane-NHS Engagement Project No: 10/4000/02 


\section{NOTES}

In future updates the relaxation techniques and manual methods reviews will be split into separate reviews on yoga, music, audio and massage and reflexology, respectively.

\section{INDEX TERMS}

\section{Medical Subject Headings (MeSH)}

Acupuncture Analgesia; Administration, Inhalation; Analgesia, Epidural [adverse effects] [methods]; Analgesia, Obstetrical [adverse effects] [ ${ }^{*}$ methods]; Analgesics [administration \& dosage] [adverse effects]; Cesarean Section [statistics \& numerical data]; Immersion; Labor Pain [ ${ }^{\star}$ therapy]; Massage; Patient Satisfaction; Relaxation Therapy [methods]; Review Literature as Topic

\section{MeSH check words}

Female; Humans; Pregnancy 